\title{
Elektrischer Transport in Manganatschichten: Einfluss von elektrischen Feldern und Licht
}

\author{
Dissertation \\ zur Erlangung des mathematisch-naturwissenschaftlichen Doktorgrades \\ „Doctor rerum naturalium“ \\ der Georg-August-Universität Göttingen
}

vorgelegt von

Christin Kalkert

aus Leverkusen

Göttingen 2013 
Referent: Prof. Dr. K. Samwer

Korreferent: Prof. Dr. V. Moshnyaga

Tag der mündlichen Prüfung: 4. April 2013 


\section{Inhaltsverzeichnis}

1 Einleitung 1

2 Manganate 5

2.1 Kristallstruktur . . . . . . . . . . . . . . . . . . . . . . . 6

2.2 Elektronische und magnetische Struktur . . . . . . . . . . . . . . 8

2.2 .1 Kristallfeld $\ldots \ldots \ldots \ldots \ldots$. . . . . . . . . . . . 8

2.2 .2 Jahn-Teller-Effekt . . . . . . . . . . . . . . . . . . 9

$2.2 .3 \quad$ Austauschmechanismen . . . . . . . . . . . . . . . . . . . . 99 9

2.3 Polaronen und elektronische Phasenseparation . . . . . . . . . . . . . . . 13

2.4 Phasendiagramm von $\mathrm{La}_{1-\mathrm{x}} \mathrm{Sr}_{\mathrm{x}} \mathrm{MnO}_{3} \ldots \ldots \ldots \ldots \ldots$

$\begin{array}{lll}3 & \text { Elektrisches Widerstandsschalten } & 17\end{array}$

3.1 Klassifizierung des Widerstandsschaltens . . . . . . . . . . . . . . . . . . . . 18

3.2 Mikroskopische Modellvorstellungen _. . . . . . . . . . . . . . . . . . . 20

3.2.1 Schottky-Barriere. . . . . . . . . . . . . . . . . . 20

3.2.2 $\quad$ Sauerstoffleerstellen-Diffusion und Elektromigration . . . . . . . . . 21

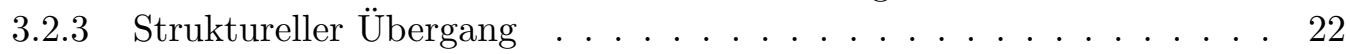

3.3 Technologische Bedeutung . . . . . . . . . . . . . . . . . . . 23

4 Experimentelle Techniken $\quad \mathbf{2 5}$

4.1 Metallorganische Aerosol Deposition . . . . . . . . . . . . . . . . . . 25

4.2 Röntgenstrukturanalyse $\ldots \ldots \ldots \ldots \ldots$. . . . . . . . . . . . . 27

4.3 Rasterelektronenmikroskopie . . . . . . . . . . . . . . . . . 28

4.4 Mikrostrukturierung $\ldots \ldots \ldots \ldots$. . . . . . . . . . . . . . . . 30

4.5 Elektronische Transportmessungen . . . . . . . . . . . . . . . . . . . 32

4.6 Magnetische Charakterisierung . . . . . . . . . . . . . . . . . . . . 33

4.7 Rastersondenmikroskopie . . . . . . . . . . . . . . . . . 33

4.8 Photoinduzierte, elektrische Transportmessungen . . . . . . . . . . . . . 35

5 Charakterisierung von $\mathrm{La}_{0,7} \mathrm{Sr}_{0,3} \mathrm{MnO}_{3} / \mathrm{Al}_{2} \mathbf{O}_{3} \quad 37$

5.1 Struktur . . . . . . . . . . . . . . . . . . . 37

5.2 Oberflächenmorphologie . . . . . . . . . . . . . . . . . . . . . 39

5.3 Elektronischer Transport und Magnetisierung . . . . . . . . . . . . . . . . . 40

5.4 Einfluss der Schichtdicke . . . . . . . . . . . . . . . . . . . . . 41

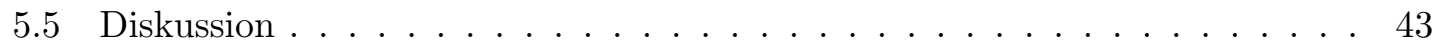

5.6 Zusammenfassung . . . . . . . . . . . . . . . . . 46

$\begin{array}{llll}6 & \text { Elektrisches Widerstandsschalten in nanokolumnaren Manganatschichten } & \mathbf{4 7}\end{array}$

6.1 C-AFM Messungen . . . . . . . . . . . . . . . . . 47

6.1 .1 Räumliche Anordnung von HRS und LRS . . . . . . . . . . . . . . . 48

6.1 .2 Wachstum der LRS-Bereiche . . . . . . . . . . . . . . . . . . 52

6.1 .3 Einfluss der Schichtdicke . . . . . . . . . . . . . . . . . . . . . 54 
$6.2 \quad$ Schaltexperimente an Mikrostrukturen . . . . . . . . . . . . . . . . . 56

$6.2 .1 \quad$ Typische Schaltcharakteristiken der mikrosktrukturierten Proben . . 56

$6.2 .2 \quad$ Variation der maximalen Schaltspannung $U_{\max } \ldots$. . . . . . . . . . 61

6.2 .3 Widerstandsschalten und Magnetowiderstand . . . . . . . . . . . . . 63

6.2 .4 Einfluss der Temperatur . . . . . . . . . . . . . . . . . . . . . 64

6.3 Diskussion . . . . . . . . . . . . . . . . . . . 67

$6.3 .1 \quad$ Energetische Betrachtungen . . . . . . . . . . . . . . . 67

6.3.2 $\quad$ Literaturvergleich: Einfluss von höherdimensionalen Defekten . . . . 70

6.3.3 Mikrostrukturen: Widerstandsnetzwerke aus Manganatsäulen . . . . 72

6.3.4 Mikroskopische Mechanismen des Widerstandsschaltens . . . . . . . 80

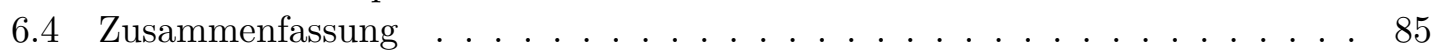

7 Photoinduzierte Änderung der ersten und dritten harmonischen Spannung 87

7.1 PPMS-Messungen der LBMO-Schicht . . . . . . . . . . . . . . . . . . . . . . . . 88

8.2 Einfluss von Laserlicht $\ldots \ldots \ldots \ldots$. . . . . . . . . . . . . . . . . . 88

7.2 .1 Temperaturabhängigkeit . . . . . . . . . . . . . . . . . . . . 89

$7.2 .2 \quad$ Zeitlicher Verlauf . . . . . . . . . . . . . . . . . . . . . . . . . . . . . . . 92

7.3 Diskussion . . . . . . . . . . . . . . . . . . . . . . . . . . . . . 94

7.4 Zusammenfassung . . . . . . . . . . . . . . . . . . 98

8 Zusammenfassung und Ausblick $\quad 101$

\begin{tabular}{ll}
\hline Literatur & 105
\end{tabular}

\begin{tabular}{ll}
\hline Publikationen & 115
\end{tabular}

\begin{tabular}{ll}
\hline Danksagung & 117
\end{tabular}

\begin{tabular}{ll}
\hline Lebenslauf & 119
\end{tabular} 


\section{Einleitung}

„Enter The OXIDES“ - so lautet der Titel eines im Jahr 2009 erschienenen Beitrags in der renommierten Nature-Zeitschrift. [1] Dieser Aufforderung wird in der vorliegenden Arbeit nachgekommen, indem sich der Erforschung von gemischt valenten Manganoxiden - den Manganaten - gewidmet wird. Manganate zählen zu den stark korrelierten Elektronensystemen, deren Verständnis für die moderne Festkörperphysik eine außerordentliche Herausforderung ist. Insbesondere die Erforschung von Grenzflächeneigenschaften bei den Oxiden ist ein „fruchtbares“ Forschungsgebiet, wie in dem Übersichtsartikel „EMERGENT PHENOMEna AT OXIDE INTERFACES“ festgestellt wird. 2] Diese Veröffentlichung befasst sich (unter anderem) mit der Entdeckung von überraschenden Zuständen an den Grenzflächen zwischen oxidischen Materialien. So fanden Ohtomo und Hwang an der Grenze zwischen zwei Isolatoren ein zweidimensionales Elektronengas mit hoher Mobilität. [3] Sogar Supraleitung wird an der Grenzfläche zweier isolierender Oxide - $\mathrm{LaAlO}_{3}$ und $\mathrm{SrTiO}_{3}$ - beobachtet. 44 In diesem Zusammenhang wurde auch ein Leitartikel (Editorial) in Nature Materials mit dem Titel „THE INTERFACE IS STILL THE DEVICE" veröffentlicht. [5] Diese Formulierung spielt auf die Nobelpreisrede (2000) von Herbert Kroemer an, der für seine Arbeit an Grenzflächen in Halbleiterheterostrukturen geehrt wurde. In dem zitierten Artikel wird er auf oxidische Grenzflächen übertragen.

Auch bei der Erforschung von Grenzflächeneigenschaften in Manganatheterostrukturen finden sich erstaunliche Ergebnisse. In einer theoretischen Arbeit von Calderón et al. wird ein metallisches und ferromagnetisches Elektronengas an der Grenzfläche zwischen zwei isolierenden und antiferromagnetischen Manganaten vorhergesagt $\left(\mathrm{La}_{0,5} \mathrm{Ca}_{0,5} \mathrm{MnO}_{3}\right.$ und $\mathrm{CaMnO}_{3}$ ). [6] Manganatsysteme zeigen reichhaltige Phasendiagramme mit einer Vielzahl von unterschiedlichen Phasen: Neben ferromagnetischen metallischen und antiferromagnetischen isolierenden Zuständen finden sich ferromagnetische isolierende und auch Ladungsund orbitale Ordnungen. Kleine Veränderungen der Kontrollparameter zeigen oft einen drastischen Einfluss auf die elektronischen, magnetischen und strukturellen Materialeigenschaften. Als externer Stimulus kann dabei neben der Dotierung auch der Einfluss von Licht oder elektrischen und magnetischen Feldern große Änderungen bewirken. [7, 8, 9] Berühmt geworden sind die Manganate durch die Entdeckung des kolossalen Magnetowiderstands vor zwanzig Jahren. [10, 11, 12, 13] Der kolossale Magnetowiderstand ist ein materialintrinsischer Effekt und beschreibt die drastische Reduktion des Probenwiderstands in einem externen Magnetfeld. 
Weiterhin wird in den Manganaten elektrisch induziertes Widerstandsschalten beobachtet. [9, 14] Hierbei handelt es sich um eine remanente Veränderung des Probenwiderstands in Abhängigkeit von der elektrischen Vorgeschichte; der Widerstand schaltet dabei zwischen zwei (oder mehr) Zuständen. Bei dem bipolaren Widerstandsschalten spielt die Polarität des angelegten Feldes die entscheidende Rolle - nur bei dem entsprechenden Betrag und der richtigen Polarität verändert die Probe remanent den Widerstand. Hierbei sind für die Manganate vermutlich die Grenzflächen entscheidend. [15]

Die Schalteffekte finden sich nicht nur in den Manganatsystemen. [16, 17] Remanentes Widerstandsschalten wird in vielen perowskitischen Oxiden, wie beispielsweise $\mathrm{SrTiO}_{3}$ und $\mathrm{SrZrO}_{3}$, beobachtet. [18, 19, 20] Weltweit widmen sich zahlreiche Forschergruppen der Untersuchung des elektrischen Widerstandsschalten. Hierbei spielt die potentielle Anwendungsmöglichkeit der Widerstandsschaltelemente als nichtflüchtige Speicherzellen eine große Rolle. Kingon [21] stellte etwa die Frage „Perovskites: Is the Ultimate MeMORY IN SIGHT?" Eine große Schwachstelle der herkömmlichen Silizium basierten FlashTechnologie liegt darin, dass sie bei der Miniaturisierung an ihre physikalischen Grenzen stößt. Gerade mit Blick auf die immer kleineren Strukturgrößen wird das Verständnis der Eigenschaften von Grenzflächen und Defekten immer wichtiger.

Aus den oben genannten Gründen untersucht diese Arbeit die Bedeutung einer definierten Defektstruktur auf das Widerstandsschalten in Manganatschichten. Zusätzlich wird als weiterer Kontrollparameter der Einfluss von Licht auf den elektronischen Transport eines Manganatsystems erforscht.

Zu Beginn werden in den Kapiteln 2 und 3 die physikalischen Fundamente zum Verständnis der anschließenden Messergebnisse vorgestellt. Hierbei werden als erstes die Grundlagen der Manganatphysik erläutert. Dazu gehört eine Einführung in die strukturellen, elektronischen und magnetischen Eigenschaften. Weiterhin werden der Superaustausch- und Doppelaustauschmechanismus vorgestellt und die für die Manganate wichtigen Konzepte der elektronischen Phasenseparation und Polaronen erklärt. Die physikalischen Grundlagen des elektrischen Widerstandsschaltens werden in Kapitel 3 erläutert. Dabei werden insbesondere die mikroskopischen Modellvorstellungen - wozu die SauerstoffleerstellenDiffusion, die Modifikation einer Schottky-Barriere und ein lokaler struktureller Übergang zählen - eingeführt.

Im Kapitel 4 werden die in dieser Arbeit verwendeten experimentellen Messmethoden und Anlagen vorgestellt. Dazu zählt die Probenpräparation von dünnen Manganatschichten mittels der Metallorganischen Aerosol Depositiontechnik. Je nach verwendetem Substrat wird das Schichtwachstum entscheidend beeinflusst. Epitaktisches Wachstum wird auf MgO-Substraten beobachtet (bei optimierten Depositionsparametern), dagegen kommt es zu polykristallinen Schichten auf $\mathrm{Al}_{2} \mathrm{O}_{3}$. Im Kapitel 5 wird die Standardcharakterisierung dieser Proben vorgestellt, wobei die epitaktischen und polykristallinen Schichten anhand der strukturellen, elektronischen und magnetischen Eigenschaften verglichen werden.

Die polykristallinen Manganatschichten zeigen eine definierte Defektstruktur. Die Körner wachsen als Säulen vom Substrat zu der Schichtoberfläche. Der Einfluss dieser Defektstruktur auf das Widerstandsschalten wird im Kapitel 6 diskutiert. Dabei werden zuerst die Messergebnisse vorgestellt, die anhand der verwendeten Methoden gegliedert sind. Zum einen wird das Widerstandsschalten mittels C-AFM auf einer sehr lokalen Skala untersucht. Ein zentrales Ergebnis dieser Messungen ist, dass das Widerstandsschalten insbesondere im Bereich der Korngrenzen lokalisiert ist. Zum anderen wurden die Manganatschichten mittels Elektronenstrahllithographie mikrostrukturiert. In den Mikrostrukturmessungen 
wird das elektronische Transportverhalten von einer Vielzahl von Manganatsäulen untersucht. Diese Ergebnisse können im Rahmen eines Widerstandsnetzwerkes aus den einzelnen Elementen (Manganatkörnern) verstanden werden. Anschließend folgt eine Diskussion des Widerstandsschaltens im Rahmen eines lokalen strukturellen Phasenübergangs im Bereich der Korngrenzen und an der Schichtoberfläche; diese Erklärungen werden insbesondere dem Modell der Sauerstoffleerstellen-Diffusion gegenübergestellt.

In Kapitel 7 werden photoinduzierte Messungen der ersten und dritten harmonischen (elektrischen) Spannung vorgestellt. Hierbei wird der Einfluss von Laseranregungen im Bereich des Metall-Isolator-Übergangs untersucht. Die Diskussion der Ergebnisse erfolgt insbesondere in Bezug auf eine Veröffentlichung von Moshnyaga et al. [22]. In dieser Arbeit wird eine erhöhte dritte harmonische Spannung im Bereich des Übergangs für einige Manganatsysteme beobachtet und der Anteil an dritter harmonischer Spannung wird mit der Konzentration der korrelierten Polaronen in Verbindung gebracht.

Zum Schluss dieser Arbeit werden die gewonnenen Erkenntnisse zusammengefasst und es folgt ein Ausblick auf die sich aus der vorliegenden Arbeit ableitenden, weiterführenden Fragestellungen. 



\section{Manganate}

Manganatsysteme bzw. gemischt valente Manganoxide sind Übergangsmetalloxide mit der allgemeinen chemischen Summenformel $\mathrm{A}_{1-x} \mathrm{~B}_{x} \mathrm{MnO}_{3}$, wobei A einem dreiwertigen Selteneerdelement (wie etwa Lanthan, Praseodym oder Neodym) und B einem zweiwertigen Erdalkalielement (z.B. Calcium, Strontium oder Barium) entspricht. Viele Manganatverbindungen zeigen einen Metall-Isolator-Übergang, in dem zusätzlich ein Übergang von Ferromagnetismus zu Paramagnetismus stattfindet (beispielsweise in Lanthan-Calcium-, Lanthan-Barium- und Lanthan-Strontium-Manganaten [10, 11, 12]). Wird im Bereich des Metall-Isolator-Übergangs ein externes Magnetfeld $H$ angelegt, so kommt es zu einer drastischen Reduktion des Widerstands $R$. Dieser Effekt wird als kolossaler Magnetowiderstand (CMR, colossal magnetoresistance) bezeichnet, der über

$$
\mathrm{CMR}=\frac{R(H=0)-R(H)}{R(H)}
$$

definiert werden kann. Ein Beispiel für die Temperaturabhängigkeit des CMR-Effekts und des Metall-Isolator-Übergangs in einem magnetischen Feld ist in Abb. 2.1 zu sehen. Bei der gezeigten $\mathrm{La}_{0,44} \mathrm{Pr}_{0,26} \mathrm{Ca}_{0,3} \mathrm{MnO}_{3}$-Probe bewirkt ein Magnetfeld von $4 \mathrm{~T}$ im Bereich des Metall-Isolator-Übergangs eine Widerstandsänderung bzw. einen CMR-Effekt von etwa $10000 \%$; in der Literatur werden sogar CMR-Beträge von über $100000 \%$ berichtet. [13]

Zusätzlich zu dem CMR-Effekt können die Manganatsysteme im metallischen, ferromagnetischen Zustand eine $100 \%$ Spinpolarisation zeigen, was sie für Anwendungen im Bereich der Spintronik interessant macht. 23] Manganate sind stark korrelierte Elektronensysteme, in denen eine Vielzahl von elektronischen, magnetischen und kristallographischen Phasen durch eine kleine Veränderung der Kontrollparameter eingestellt werden können. Als Kontrollparameter kann die Dotierung, das Magnetfeld, die Temperatur oder auch der Druck dienen, wobei in dieser Arbeit der Effekt des elektrischen Feldes (Kap. 6) und der Einfluss von Licht (Kap. 7) insbesondere auf den elektrischen Transport untersucht werden.

Die nachfolgenden Ausführungen geben eine kurze Einführung in die physikalischen Grundlagen dieser vielfältigen Manganatsysteme. Besondere Relevanz erhält daher einerseits die Zusammenfassung von Gross und Marx [24] und die Fachliteratur von Dagotto [25] als auch von Tokura [26]. 


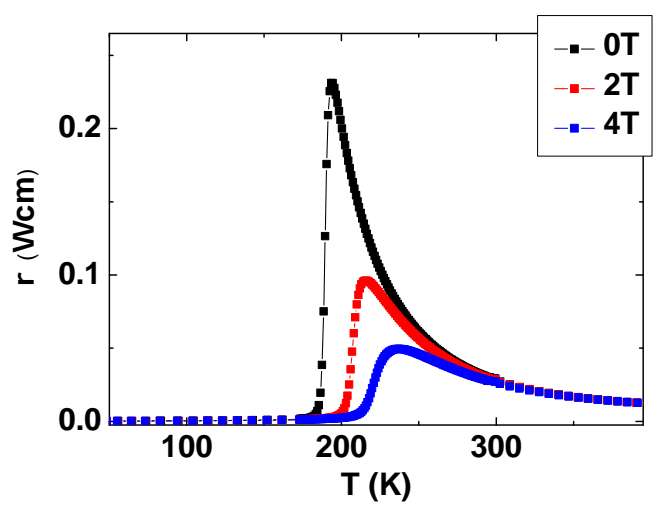

(a) Abhängigkeit des spezifischen Widerstands von der Temperatur

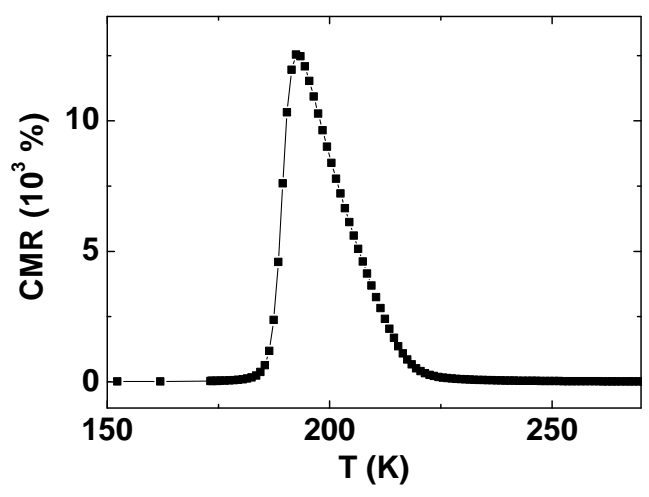

(b) Temperaturabhängigkeit des CMR

Abb. 2.1: Metall-Isolator-Übergang und CMR-Effekt in $\mathrm{La}_{0,44} \mathrm{Pr}_{0,26} \mathrm{Ca}_{0,3} \mathrm{MnO}_{3}$.

\subsection{Kristallstruktur}

Manganate kristallisieren in verschiedenen Modifikationen der Perowskit-Kristallstruktur mit der Summenformel $\mathrm{ABO}_{3}$. Die A-Gitterplätze werden (in den hier behandelten Verbindungen) von dreiwertigen Selteneerdionen und zweiwertigen Kationen eingenommen. Die B-Gitterplätze werden von den drei- bis vierwertigen Manganionen besetzt, wobei das Verhältnis von $\mathrm{Mn}^{3+}$ zu $\mathrm{Mn}^{4+}$ durch das Verhältnis der zwei- zu dreiwertigen A-Platz Gitterionen bestimmt ist. Die kubische Perowskit-Kristallstruktur ist in Abb. 2.2 gezeigt; auch die oktaedrische Umgebung der Manganionen durch die Sauerstoffionen ist skizziert.
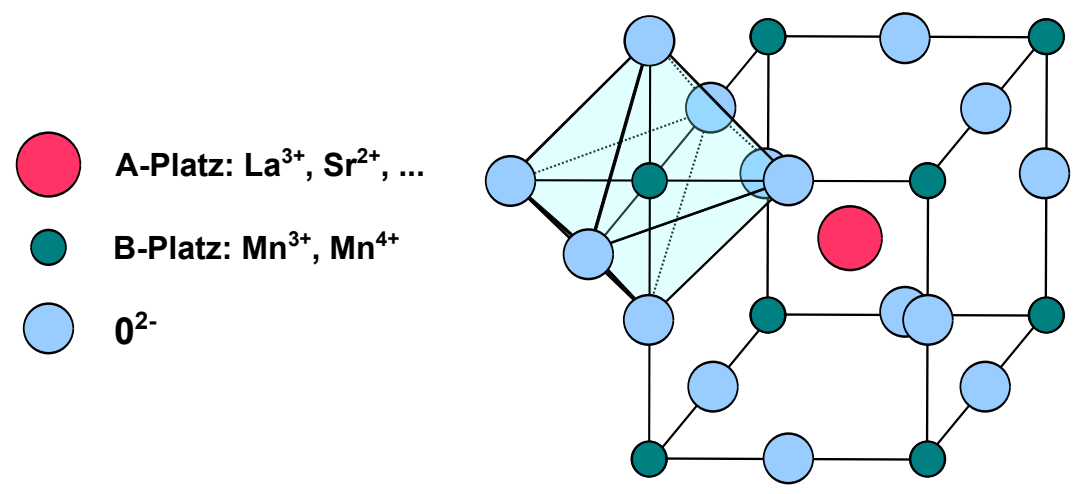

Abb. 2.2: Schema der quasi-kubischen Perowskitstruktur.

In den unterschiedlichen Manganatsystemen können je nach chemischer Zusammensetzung verschiedene, niedersymmetrische Modifikationen der Perowskitstruktur auftreten. Hierbei können die Sauerstoffoktaeder verdreht und verkippt sein. Dadurch ändern sich die für den elektronischen Transport entscheidenden Abstände und Winkel zwischen den Mn-O-MnIonen. Ein Maßstab für die Modifikation der Kristallstruktur aufgrund der unterschiedlichen Verhältnisse der Ionenradien der A- und B-Ionen ist durch den Goldschmidtschen Toleranzfaktor gegeben

$$
t=\frac{d_{\mathrm{A}-\mathrm{O}}}{\sqrt{2} \cdot d_{\mathrm{B}-\mathrm{O}}} \cong \frac{1}{\sqrt{2}} \cdot \frac{\left\langle r_{\mathrm{A}}\right\rangle+r_{\mathrm{O}}}{\left\langle r_{\mathrm{B}}\right\rangle+r_{\mathrm{O}}}
$$




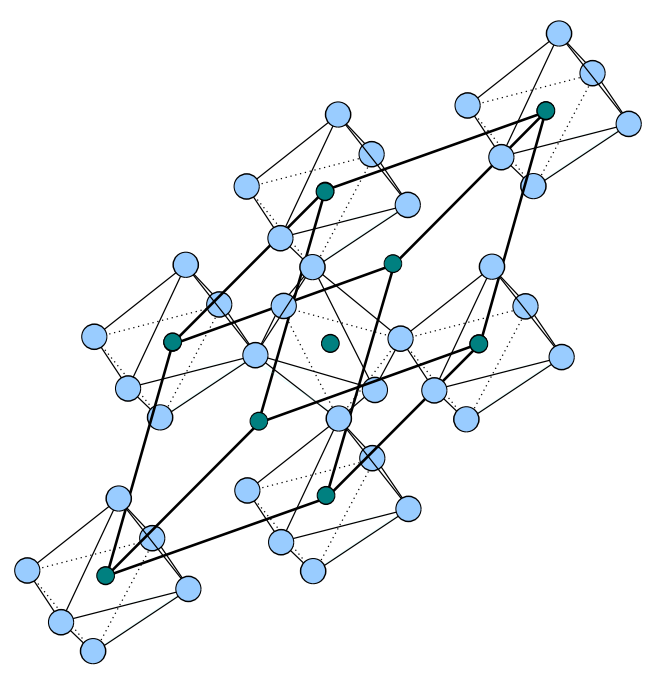

(a) rhomboedrische $\mathrm{R} \overline{3} \mathrm{c}-\mathrm{S}$ truktur

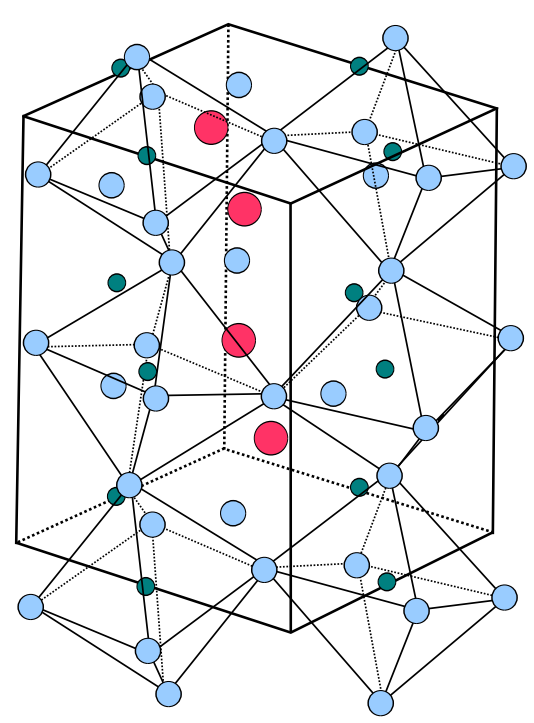

(b) orthorhombische Pnma-Struktur

Abb. 2.3: Niedersymmetrische Modifikationen der Perowskitstruktur nach Tokura [28].

Dabei ist $d_{\mathrm{A}-\mathrm{O}}$ bzw. $d_{\mathrm{B}-\mathrm{O}}$ der Abstand zwischen dem jeweiligen Gitterplatz- und Sauerstoffion. Für die ideale, kubische Perowskitstruktur gilt $d_{\mathrm{A}-\mathrm{O}} / d_{\mathrm{B}-\mathrm{O}}=\sqrt{2}$ und der Toleranzfaktor ist $t=1$. Die Bindungslängen $d_{\mathrm{A}-\mathrm{O}}$, B-O sind näherungsweise durch die Summe der zugehörigen Ionenradien $r_{\mathrm{A}}$, B, o gegeben. ${ }^{1}$

Liegt der Toleranzfaktor zwischen $1<t<0,96$, so wird die rhomboedrische Perowskitstruktur $(\mathrm{R} \overline{3} \mathrm{c})$ begünstigt; für $t<0,96$ wird die orthorhombische Pnma-Struktur bevorzugt. [26] Die rhomboedrische R $\overline{3}$ c-Struktur ist in Abb. 2.3(a) dargestellt. Hierbei sind entlang der drei pseudokubischen Raumrichtungen die Sauerstoffoktaeder jeweils alternierend verkippt. Die Pnma-Kristallstruktur ist in Abb. 2.3(b) gezeigt. Die Sauerstoffoktaeder sind in zwei Raumrichtungen (der pseudokubischen Darstellung) alternierend verkippt, entlang der dritten Raumrichtung sind die Oktaeder in die gleiche Richtung geneigt. Ist der Toleranzfaktor kleiner als eins, bedeutet das eine Abweichung des Bindungswinkels zwischen den Mn-O-Mn-Ionen von $180^{\circ}$. Dieser ist für den elektronischen Transport entscheidend und eine Abweichung ist tendenziell mit einer geringeren Mobilität der Ladungsträger verbunden.

Der Toleranzfaktor gibt lediglich ein Maß für die Verspannung der Struktur aufgrund unterschiedlich großer A- und B-Ionen an. Viele für die Struktur der Manganate wichtigen Aspekte bleiben unberücksichtigt. So wird zur Berechnung des Toleranzfaktors lediglich der mittlere Radius der A- und B-Platz Gitteratome verwendet. Gibt es stark unterschiedliche Ionenradien, kann das zu einer veränderten Struktur führen. Des Weiteren zeigten Mitchell et al., dass eine Auslagerung in verschiedenen Sauerstoffdrücken bei gleich dotierten Manganatproben in unterschiedlichen strukturellen Phasen resultieren kann. [29] Auch der Jahn-Teller-Effekt führt zu einer Modifikation der Kristallstruktur, dieser wird im nächsten Abschnitt erläutert.

\footnotetext{
${ }^{1}$ Die Ionenradien der Manganionen sind gegeben durch $r_{\mathrm{Mn}^{3+}}=0,645 \AA$ und $r_{\mathrm{Mn}^{4+}}=0,53 \AA$ (sechsfach koordiniert, high spin state), für typische A-Gitterplatzionen gilt $r_{\mathrm{La}^{3+}}=1,36 \AA, r_{\mathrm{Sr}^{2+}}=1,44 \AA$ und $r_{\mathrm{Ba}^{2+}}=1,61 \AA$ (zwölffach koordiniert). Der Radius des $\mathrm{O}^{2-}$-Ions beträgt $r_{\mathrm{O}^{2-}}=1,4 \AA$ (sechsfach koordiniert). 27]
} 


\subsection{Elektronische und magnetische Struktur}

Um die elektronische und magnetische Struktur der Manganate beschreiben zu können, muss die Elektronenkonfiguration der beteiligten Ionen $\left(\mathrm{A}_{1-x}^{3+} \mathrm{B}_{x}^{2+} \mathrm{Mn}_{1-x}^{3+} \mathrm{Mn}_{x}^{4+} \mathrm{O}_{3}^{2-}\right)$ analysiert werden. Bei den A-, B- und O-Ionen ist die äußere Schale abgeschlossen, diese Ionen liegen in Edelgaskonfiguration vor. ${ }^{2}$ Für die Manganionen gilt $\mathrm{Mn}^{3+}:[\mathrm{Ar}] 3 \mathrm{~d}^{4}$ und $\mathrm{Mn}^{4+}:[\mathrm{Ar}] 3 \mathrm{~d}^{3}$. Die d-Orbitale des Manganions sind daher für die Erklärung und das Verständnis der elektronischen und magnetischen Eigenschaften der Manganate von entscheidender Bedeutung. Ein Schema der relevanten d-Orbitale findet sich in Abb. 2.4.

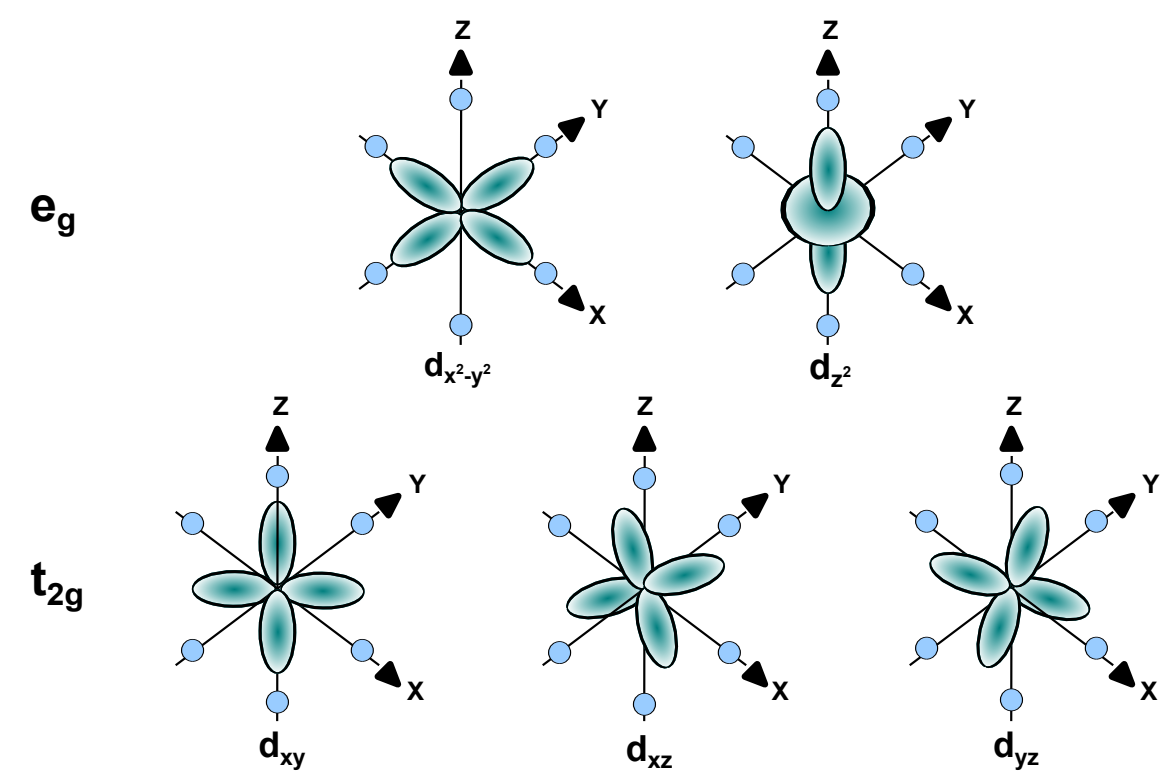

Abb. 2.4: Schema der d-Orbitale. Die blauen Punkte deuten die Positionen der Sauerstoffionen im umgebenden Kristallgitter an.

\subsubsection{Kristallfeld}

Für ein freies Ion sind die fünf d-Orbitale entartet. In einer Kristallfeldumgebung wird die Entartung der d-Orbitale aufgrund von Coulomb-Abstoßung aufgehoben. Je nach Lage der Orbitale in Relation zu den umgebenden Sauerstoffionen bzw. -Orbitalen sind sie energetisch begünstigt oder benachteiligt. Das die Manganionen umgebende Kristallfeld ist durch die in Abb. 2.4 eingezeichneten Positionen der Sauerstoffionen angedeutet. Daraus ist ersichtlich, dass die $\mathrm{e}_{g^{-}}$Orbitale $\left(\mathrm{d}_{x^{2}-y^{2}}\right.$ und $\left.\mathrm{d}_{z^{2}}\right)$ energetisch ungünstiger sind, da sie in Richtung der Sauerstoffionen zeigen. Dagegen sind die $t_{2 g}$-Orbitale, zu denen $\mathrm{d}_{x y}, \mathrm{~d}_{x z}$ und $\mathrm{d}_{y z}$ zählen, energetisch günstiger angeordnet. Die energetische Aufspaltung zwischen den $\mathrm{t}_{2 g}$ und $\mathrm{e}_{g}$-Orbitalen beträgt etwa $\Delta_{C F} \approx 1 \mathrm{eV}$. [26]

Bei einem $\mathrm{Mn}^{4+}$-Ion müssen drei Elektronen auf die 3d-Orbitale verteilt werden; diese besetzen die drei $t_{2 g}$-Orbitale mit parallelem Spin gemäß der Hundschen Regeln. Bei einem $\mathrm{Mn}^{3+}$-Ion muss ein weiteres Elektron untergebracht werden. Dieses kann entweder mit antiparallelem Spin zu den anderen Elektronen in ein $\mathrm{t}_{2 g}$-Orbital eingebaut werden oder mit parallelem Spin ein $\mathrm{e}_{g}$-Orbital besetzen. Bei den Manganaten liegt die Hundsche Kopplung

\footnotetext{
${ }^{2}$ Dieses gilt für die in dieser Arbeit untersuchten Manganatsysteme $\mathrm{La}_{1-x} \mathrm{Sr}_{x} \mathrm{MnO}_{3}$ und $\mathrm{La}_{1-x} \mathrm{Ba}_{x} \mathrm{MnO}_{3}$. $\mathrm{Nb}^{3+}$ dagegen liegt nicht in Edelgaskonfiguration vor und kann zum magnetischen Moment der Probe beitragen.
} 
typischerweise bei $J_{H}=2 \ldots 3 \mathrm{eV}$, [26] womit sie größer als $\Delta_{C F}$ ist. Daraus folgt, dass in den Manganaten der high spin state, in dem drei $\mathrm{t}_{2 g^{-}}$und ein $\mathrm{e}_{g}$-Orbital besetzt sind und die Spins parallel zueinander ausgerichtet sind, bevorzugt wird (Gesamtspin $S=2$ ).

\subsubsection{Jahn-Teller-Effekt}

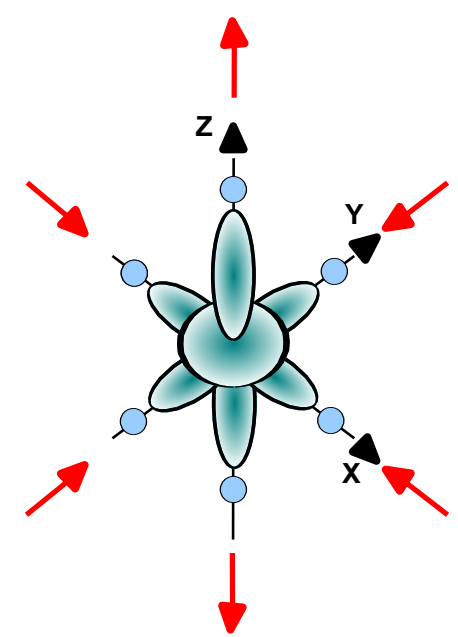

(a) Jahn-Teller-Effekt

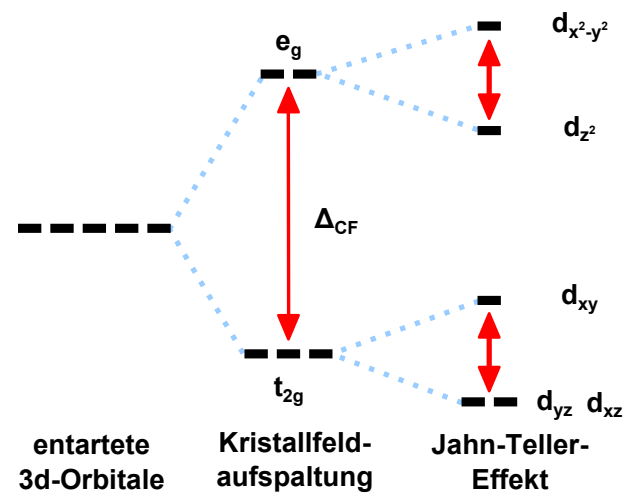

(b) Energieniveauschema nach Tokura [28]

Abb. 2.5: Schema des Jahn-Teller-Effekts und der Energieniveaus der 3d-Orbitale.

$\mathrm{Zu}$ einer weiteren Aufspaltung der Energieniveaus der d-Orbitale kann es aufgrund des Jahn-Teller-Effekts kommen. Durch eine Reduzierung der Kristallsymmetrie - etwa durch eine Elongation des Oktaeders entlang der z-Achse und eine Kompression entlang der xund y-Achse - wird der Überlapp benachbarter Orbitale verändert. Dafür muss zwar elastische Verzerrungsenergie aufgebracht werden, allerdings verringert sich der Überlapp des $\mathrm{d}_{z^{2}}$-Orbitals des Mangans mit dem Sauerstoff p-Orbital, wodurch die Coulomb-Abstoßung verringert wird. Dieses kann insgesamt zu einem Energiegewinn führen, wenn ein $\mathrm{e}_{\mathrm{g}}$-Orbital besetzt ist. Da bei $\mathrm{Mn}^{4+}$-Ionen keine Elektronen in den $\mathrm{e}_{g}$-Orbitalen sind, bringt eine Jahn-Teller-Verzerrung keinen Energiegewinn. Die Veränderung der oktaedrischen Umgebung aufgrund des Jahn-Teller-Effekts für ein $\mathrm{Mn}^{3+}$-Ion ist in Abb. 2.5(a) gezeigt, das zugehörige Energieniveauschema in Abb. 2.5(b).

Über die Dotierung wird das Verhältnis von $\mathrm{Mn}^{3+}$ - und $\mathrm{Mn}^{4+}$-Ionen bestimmt. Für Manganatproben mit einem großen Anteil von $\mathrm{Mn}^{3+}$-Ionen kann eine gemeinsame Anordnung der Jahn-Teller-Ionen mechanische Verspannung abbauen. Ein Beispiel für diesen als kooperativen Jahn-Teller-Effekt bezeichneten, strukturellen Übergang ist die orbitale Ordnung in $\mathrm{LaMnO}_{3}$, diese ist in Abb. 2.6 gezeigt. Die beschriebene Situation ist ein Beispiel für einen statischen Jahn-Teller-Effekt, aber auch ein dynamischer Jahn-Teller-Effekt wird beobachtet. Die Verzerrung kann von einer Achse zu einer anderen Achse dynamisch wechseln oder ein Jahn-Teller-Ion kann (bei einer gemischten Valenz von $\mathrm{Mn}^{3+}$ und $\mathrm{Mn}^{4+}$ ) von Gitterplatz zu Gitterplatz hüpfen. [30] Dieses wird als Jahn-Teller-Polaron bezeichnet, auf den polaronischen Transport wird im Abschnitt 2.3 eingegangen.

\subsubsection{Austauschmechanismen}

Wie eingangs beschrieben, spielt das Manganion eine Schlüsselrolle für die elektronische und magnetische Struktur der Manganate. Das magnetische Moment des Manganats wird 


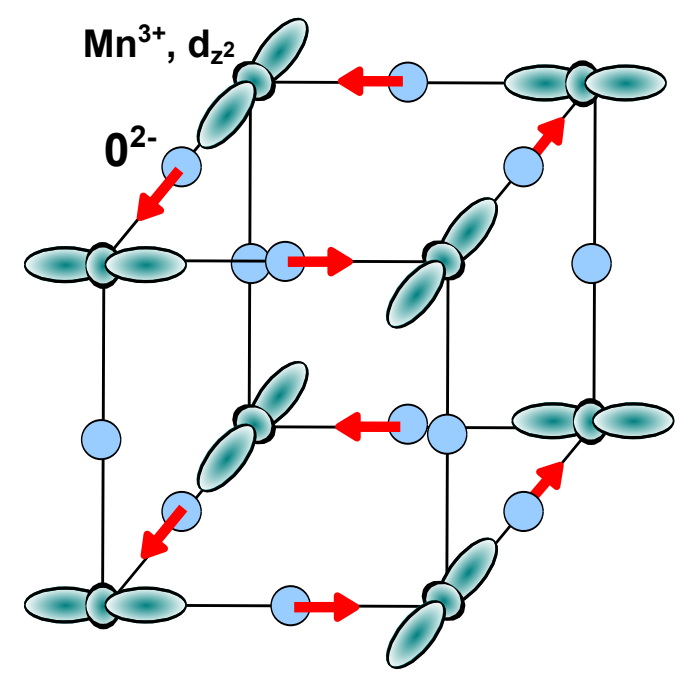

Abb. 2.6: Schema der orbitale Ordnung in $\mathrm{LaMnO}_{3}$ aufgrund des Jahn-Teller-Effekts nach Gross und Marx [24]. Die Pfeile deuten die Auslenkungen der Sauerstoffionen an.

dabei durch das Spinmoment des Manganions bestimmt, ${ }^{3}$ da bei den 3d-Übergangsmetallen der Bahndrehimpuls in oktaedrischer Kristallfeldumgebung "gequencht" ist. 24] Wechselwirkungen zwischen den Manganionen, die zu einer magnetischen Ordnung führen können, geschehen indirekt über das p-Orbital des Sauerstoffions, das sich zwischen den Manganionen befindet. Die $t_{2 g}$-Elektronen sind lokalisiert, die Wechselwirkung der Manganionen untereinander kann anhand von Austauschprozessen der $\mathrm{e}_{g}$-Elektronen verstanden werden, wobei die Spinrichtung erhalten bleibt.

Für die Physik der Manganate sind insbesondere der Superaustausch und der Doppelaustausch fundamentale Mechanismen. Zu diesen beiden Mechanismen werden an dieser Stelle einige qualitative Überlegungen und vereinfachte Darstellungen vorgestellt; diese können die Komplexität der Manganat-Physik nicht vollständig erfassen. Für eine grundlegendere Beschreibung dieser Mechanismen sei daher auf das Werk von Dagotto [25] verwiesen.

\section{Superaustausch}

Um die energetisch günstigste Anordnung für die Elektronenspins der 3d-Orbitale zu ermitteln, müssen folgende Energiebeiträge berücksichtigt werden. Zum einen gewinnen die Elektronen aufgrund von Delokalisierung kinetische Energie. Als Maßstab hierfür wird die Hüpfamplitude $t>0$ eingeführt, wobei $t$ als Maß für den orbitalen Überlapp der Ionen angesehen werden kann. Zum anderen ist die lokale Coulomb-Abstoßung $U>0$ die zweite wichtige Größe. Störungstheoretische Rechnungen zweiter Ordnung führen auf eine Kopplungsenergie $\Delta E$, [30] für die gilt

$$
\Delta E \propto-\frac{t^{2}}{U}
$$

In Abb. 2.7 ist ein Schema des Superaustausch-Modells für ein Ein-Niveau-System zu sehen, in dem ausschließlich ein $\mathrm{e}_{g}$-Orbital pro Manganion berücksichtigt wird. Jedes Orbital

\footnotetext{
${ }^{3}$ Für eine $\mathrm{La}_{0,7} \mathrm{Sr}_{0,3} \mathrm{MnO}_{3}$-Probe berechnet sich das magnetische Moment folgendermaßen: Durch die Dotierung ist der Anteil der $\mathrm{Mn}^{3+}$ und $\mathrm{Mn}^{4+}$-Ionen gegeben, diese haben $\mathrm{S}_{M n^{3+}}=2$ und $\mathrm{S}_{M n^{4+}}=3 / 2$. Damit ergibt sich $m=\mu_{\mathrm{B}}\left(L+g_{\mathrm{s}} \cdot S\right)=\mu_{\mathrm{B}} g_{\mathrm{s}} S=\mu_{\mathrm{B}} g_{\mathrm{s}}\left(0,7 \cdot S_{\mathrm{Mn}^{3+}}+0,3 \cdot S_{\mathrm{Mn}^{4+}}\right)=\mu_{\mathrm{B}} \cdot 3,7$ (mit $\left.g_{\mathrm{s}}=2\right)$.
} 


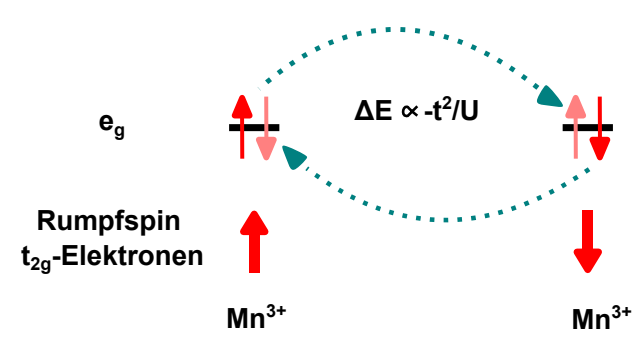

(a) antiferromagnetisch

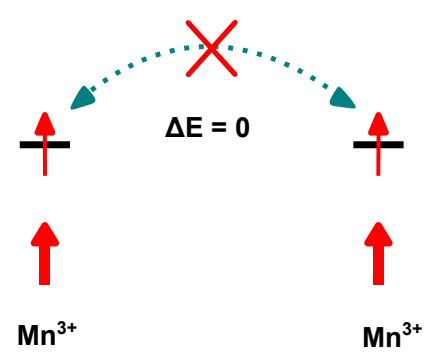

(b) ferromagnetisch

Abb. 2.7: Superaustausch im Ein-Niveau-Schema (ohne Sauerstoffionen) nach Ref. [24, 30].

ist einfach besetzt. Dieses entspricht einem vereinfachten Modell etwa für das $\mathrm{LaMnO}_{3}$ System (in $\mathrm{LaMnO}_{3}$ sind nur $\mathrm{Mn}^{3+}$-Ionen vorhanden, also eine einfache Besetzung der $\mathrm{e}_{g^{-}}$ Orbitale).

Sind die Elektronenspins benachbarter Ionen in die gleiche Richtung bzw. ferromagnetisch ausgerichtet, verbietet das Pauli-Prinzip den Elektronenaustausch. Die Energie des Systems kann nicht durch Delokalisierung abgesenkt werden (Abb. 2.7(b)). Bei einer antiferromagnetischen Anordnung dagegen ist der Austausch von Elektronen zwischen benachbarten Manganionen erlaubt, womit die antiferromagnetische Ordnung der $\mathrm{e}_{g}$-Elektronenspins energetisch begünstigt ist (Abb. 2.7(a)). Die starke Hundsche Kopplung richtet die Spins der lokalisierten $t_{2 g}$-Elektronen entsprechend der $\mathrm{e}_{g}$-Elektronenspins aus. Es kommt zu einer antiferromagnetischen Ordnung. Die Elektronenaustauschprozesse beschreiben hier lediglich einen „virtuellen Hüpfprozess“ oder kurzzeitigen Elektronenaustausch, der nicht mit elektronischem Transport verbunden ist. Die Ursache hierfür ist, dass $U \gg t$; eine Doppelbesetzung der Orbitale ist also energetisch ungünstig (Stichwort: Mott-HubbardIsolator).

Die Beschreibung im Ein-Niveau-Schema mit einfach besetzten Orbitalen führt zu einer antiferromagnetischen Kopplung. Dabei wird eine ganze Reihe von vereinfachenden Annahmen gemacht. Es wird lediglich ein Orbital pro magnetischem Ion betrachtet, die Sauerstoffionen bleiben unberücksichtigt. Auch die Form bzw. die geometrische Ausrichtung der Orbitale spielt keine Rolle. Eine detailliertere Vorhersage für den magnetischen Ordnungszustand kann mit den Regeln von Goodenough, Kanamori und Anderson (GKA-Regeln) getroffen werden, siehe hierzu Ref. [24].

\section{Doppelaustausch}

Bei einer gemischten Valenz von Manganionen $\left(\mathrm{Mn}^{3+}\right.$ und $\left.\mathrm{Mn}^{4+}\right)$ wird oft ein ferromagnetischer Grundzustand gefunden, der durch den Doppelaustausch-Mechanismus verstanden werden kann. Während beim Superaustausch nur „virtuelles Hüpfen“ angenommen wird, geht es im Folgenden um elektronischen Transport, der mit einer ferromagnetischen Ordnung verbunden ist. Ein Schema für den Doppelaustausch-Mechanismus ist in Abb. 2.8 gezeigt. Hier sind zwei benachbarte $\mathrm{Mn}^{3+}$ - und $\mathrm{Mn}^{4+}$-Ionen skizziert. Das $\mathrm{e}_{g}$-Elektron des (linken) $\mathrm{Mn}^{3+}$-Ions kann in das benachbarte p-Orbital des Sauerstoffs hüpfen und das Elektron des Sauerstoff p-Orbitals hüpft in das $\mathrm{e}_{g}$-Orbital des (rechten) $\mathrm{Mn}^{4+}$-Ions. Dadurch kommt es zu einem Energiegewinn an kinetischer Energie. Sind die $t_{2 g}$-Elektronen der benachbarten Mn-Ionen antiparallel ausgerichtet, so verbietet zwar nicht wie beim Superaustausch das Pauli-Prinzip den Hüpfprozess. Es müsste aber die in Manganaten sehr 


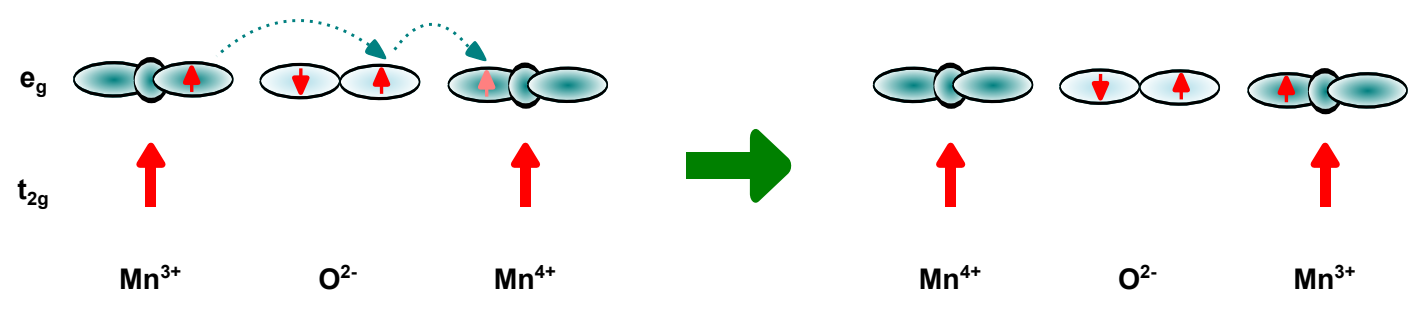

Abb. 2.8: Funktionsskizze des Doppelaustausch-Mechanismus nach Gross und Marx [24].

große Hundsche Kopplungsenergie zwischen dem $\mathrm{e}_{g^{-}}$und den $\mathrm{t}_{2 g}$-Elektronen aufgebracht werden. Damit ist die ferromagnetische Ausrichtung der $t_{2 g}$-Elektronenspins benachbarter Ionen energetisch günstiger.

Im Rahmen des Doppelaustausch-Mechanismus kann auch eine erste phänomenologische Erklärung des CMR-Prozesses gegeben werden. Der kinetische Energiegewinn der Elektronen ist proportional zum Winkel $\left(\theta_{i j}\right)$ zwischen den $t_{2 g}$-Rumpfspins von benachbarten Manganionen. Dieses kann durch eine effektive Hüpfamplitude $t_{\text {eff }}$ berücksichtigt werden. [31] Diese ist gegeben durch

$$
t_{\mathrm{eff}}=t \cdot \cos \left(\frac{\theta_{i j}}{2}\right)
$$

In der Nähe der Curie-Temperatur nimmt der Ordnungsgrad der Rumpfspins ab, wodurch der Doppelaustausch und der damit verbundene elektronische Transport geschwächt werden. Ein Magnetfeld stellt die ferromagnetische Ordnung im Bereich von $\mathrm{T}_{\mathrm{C}}$ wieder her, wobei gleichzeitig der Widerstand reduziert wird. Damit ergibt sich eine qualitative Erklärung für den im Bereich des Übergangs erhöhten CMR. Bei Temperaturen unterhalb der Curie-Temperatur im ferromagnetischen Bereich sind die Rumpfspins der Manganionen weitestgehend ausgerichtet. Dementsprechend ist der CMR-Effekt klein. In der paramagnetischen Phase oberhalb des Metall-Isolator-Übergangs werden sehr große Magnetfelder benötigt, um die Spins auszurichten. Damit kann qualitativ die Temperaturabhängigkeit des CMR und der Zusammenhang zwischen dem Übergang von Metall zu Isolator und Ferromagnetismus zu Paramagnetismus verstanden werden. Allerdings können im Rahmen des Doppelaustausch-Modells viele experimentelle Messungen nicht erklärt werden. Im Folgenden werden einige dieser Ergebnisse vorgestellt.

Millis et al. zeigen, dass das Doppelaustausch-Modell die Absolutwerte des spezifischen Widerstands falsch vorhersagt und die theoretisch erwartete, temperaturabhängige Widerstandskurve nicht mit den experimentellen Messungen für $T<T_{\mathrm{C}}$ oder den Messungen im Magnetfeld übereinstimmt. [32] Auch ist die berechnete Dotierungsabhängigkeit etwa im $\mathrm{La}_{1-x} \mathrm{Sr}_{x} \mathrm{MnO}_{3}$-System zu schwach. [32] Des Weiteren finden Zhao et al. eine Temperaturabhängigkeit der Curie-Temperatur für verschiedene Sauerstoffisotope in $\mathrm{La}_{0,8} \mathrm{Ca}_{0,2} \mathrm{MnO}_{3+y}$-Proben. [33]

Zusätzlich können die großen Unterschiede für die Manganatsysteme mit unterschiedlichen A-Platz-Gitterionen nicht erklärt werden. Ein Beispiel hierfür ist die Temperaturabhängigkeit des spezifischen Widerstands $\rho(T)$ in der paramagnetischen Phase von $\mathrm{La}_{0,7} \mathrm{Ca}_{0,3} \mathrm{MnO}_{3}$ und $\mathrm{La}_{0,7} \mathrm{Sr}_{0,3} \mathrm{MnO}_{3}$. Für das $\mathrm{La}_{0,7} \mathrm{Ca}_{0,3} \mathrm{MnO}_{3}$-System ist $d \rho / d T<0$, während bei $\mathrm{La}_{0,7} \mathrm{Sr}_{0,3} \mathrm{MnO}_{3} d \rho / d T>0$ bleibt. [24] Darüber hinaus gibt es Phasen, die mit den beschriebenen Austauschmechanismen nicht zu erklären sind. Beispielsweise findet 
sich in dem Phasendiagramm von $\mathrm{La}_{1-x} \mathrm{Sr}_{x} \mathrm{MnO}_{3}$, das im Abschnitt 2.4 vorgestellt wird, ein antiferromagnetischer, metallischer Bereich für $\mathrm{La}_{0,46} \mathrm{Sr}_{0,54} \mathrm{MnO}_{3}$. [34]

Die experimentellen Ergebnisse zeigen, dass der Superaustausch- und der DoppelaustauschMechanismus alleine nicht ausreichen, um den CMR-Effekt und die vielfältigen Phasendiagramme zu beschreiben.

\subsection{Polaronen und elektronische Phasenseparation}

Weiterführende Konzepte schließen eine elektronische Phasenseparation und den Einfluss von Polaronen auf die elektronischen, magnetischen und strukturellen Eigenschaften der Manganate mit ein. Diese für die Manganat-Physik wichtigen Konzepte werden im Folgenden kurz eingeführt.

\section{Polaronen}

Ein Polaron ist ein Elektron (oder Loch) umgeben von einer „Wolke“, in der - durch den Ladungsträger induziert - die Eigenschaften des Kristalls verändert sind. Ein Beispiel hierfür ist ein Gitterpolaron, in dem der Ladungsträger von einer lokalen Gitterverzerrung umgeben ist. Das polaronische Verzerrungsfeld kann auch Spin-Anteile haben, wobei das Verzerrungsfeld veränderte magnetische Eigenschaften zeigt, oder auch dielektrische und orbitale Anteile. Zur Verdeutlichung sind Beispiele für Polaronen in Abb. 2.9 schematisch skizziert. Je nach Größe des Verzerrungsfelds wird zwischen kleinen und großen Polaronen unterschieden. Bei den kleinen Polaronen liegt der Radius des Verzerrungsfeldes in der Größenordnung eines Gitterabstandes, für große Polaronen ist er wesentlich größer. In den Manganaten spielt das Jahn-Teller-Polaron (JT-Polaron) eine entscheidende Rolle. Hierbei beruht das Verzerrungsfeld auf der Deformation des $\mathrm{MnO}_{6}$-Oktaeders (wie im Abschnitt 2.2.2 beschrieben). Im paramagnetischen, isolierenden Bereich wird die elektrische Leitfähigkeit oft durch die thermisch aktivierte Bewegung von kleinen Polaronen (JTPolaronen) beschrieben. [35, 36]

Die Ergebnisse von Neutronen- und Röntgenbeugungsexperimenten zeigen, dass im Bereich des Metall-Isolator-Übergangs nicht nur individuelle Polaronen vorhanden sind, sondern auch „korrelierte Polaronen“. [25, 37, 38, 39] Diese können in Form von ladungsgeordneten CE-Clustern realisiert sein. Auch Messungen der elektrischen, dritten harmonischen Spannungen finden einen erhöhten Anteil an korrelierten Polaronen im Bereich des Metall-

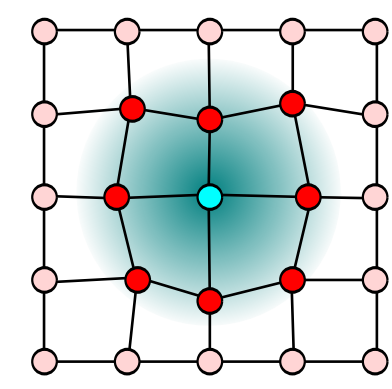

(a) Gitterpolaron

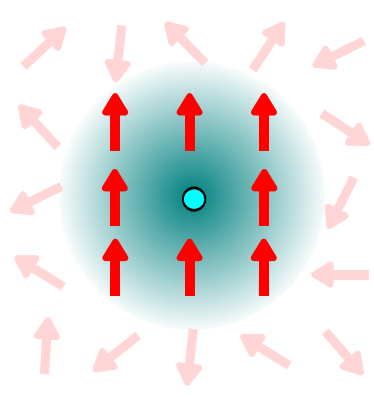

(b) Spinpolaron

Abb. 2.9: Beispiele verschiedener Polaronen nach Ref. [25]. 
Isolator-Übergangs (etwa in $\mathrm{La}_{0,75} \mathrm{Ca}_{0,25} \mathrm{MnO}_{3}$-Proben). 22 Im Gegensatz dazu wurde (mit dieser Methode) keine signifikante Veränderung des $\mathrm{U}_{3 \omega}$-Signals in dem „prototypischen Doppelaustausch-System “ 28] $\mathrm{La}_{0,7} \mathrm{Sr}_{0,3} \mathrm{MnO}_{3}$ gefunden. Auf weitere Details dieser Veröffentlichung [22] und der Messung von dritten harmonischen Spannungen wird im Kapitel 7 eingegangen.

Polaronische Korrelation und deren Rolle im CMR- und CER-Effekt ${ }^{4}$ werden von Jooss et al. im $\mathrm{Pr}_{1-x} \mathrm{Ca}_{x} \mathrm{MnO}_{3}$-System untersucht. [36] In einem Transmissionselektronenmikroskop wurde ein elektrisch induzierter Übergang von einem polaronischen Festkörper zu einer polaronischen Flüssigkeit beobachtet. Dabei beruht die polaronische Flüssigkeit auf dynamischen, kurzreichweitigen Korrelationseffekten und der polaronische Festkörper auf der statischen, langreichweitigen Ordnung der Jahn-Teller-Verzerrungen.

Ein weiteres Modell zur Erklärung des CMR-Effekts stammt von Alexandrow et al. und beruht auf dem Aufbrechen von Bipolaronen. Die Bipolaronen der paramagnetischen Phase werden dabei in der ferromagnetischen Phase aufgebrochen, wobei der CMR-Effekt durch diesen Ladungsträgerdichte-Kollaps (charge carrier density collapse) erklärt wird. [40, 41]

\section{Elektronische Phasenseparation}

Der Begriff der elektronischen Phasenseparation bedeutet, dass auch in einem chemisch homogenen System gleichzeitig konkurrierende Phasen ${ }^{5}$ vorliegen. Die Phasen können sich aufgrund von Symmetriebrechung bezüglich ihrer Spin-, Orbitalen- und/oder LadungsOrdnung unterscheiden. Haben die Phasen allerdings unterschiedliche Ladungsträgerdichten, so müssen die einzelnen Bereiche sehr klein sein (in der Größenordnung von Nanometern), da bei makroskopischen Bereichen die Coulomb-Abstoßung zu groß wäre. [24]

Inhomogenitäten bzw. eine Phasenseparation werden sowohl theoretisch als auch experimentell beobachtet. Monte-Carlo-Simulationen finden eine elektronische Phasenseparation mit unterschiedlichen Ladungsträgerkonzentrationen bzw. verschiedener Beweglichkeit der Ladungsträger, etwa im Ein-Orbital- und Zwei-Orbital-Modell. [42, 43] Die Domänengröße der Phasen liegt dabei in der Größenordnung von einigen Nanometern.

Experimentelle Messungen finden nebeneinander vorliegende Phasen auf einer Vielzahl von Längenskalen. So wurden im vorherigen Abschnitt Röntgen- und Neutronenstreuexperimente vorgestellt, in denen ladungsgordnete Cluster (bzw. korrelierte Polaronen) gefunden wurden. Dai et al. geben eine Kohärenzlänge zwischen $13 \ldots 28 \AA$ für die Polaronen-Korrelationen in $\mathrm{La}_{1-x} \mathrm{Ca}_{x} \mathrm{MnO}_{3}$-Proben $(x=0,25 \ldots 0,3)$ an. [37] Mittels Rastersondenmikroskopie und -spektroskopie (STM/STS scanning tunneling microscopy and spectroscopy) finden Fäth et al. in $\mathrm{La}_{1-x} \mathrm{Ca}_{x} \mathrm{MnO}_{3}(x \approx 0,3)$ nur wenig unter der Curie-Temperatur Bereiche mit unterschiedlichen Tunnelleitfähigkeiten. [44] An $\mathrm{La}_{1-x-y} \mathrm{Pr}_{y} \mathrm{Ca}_{x} \mathrm{MnO}_{3}$-Proben wurden - mittels Elektronenmikroskopie - sogar submikrometer große Phasen gefunden. [45]

Liegen gleichzeitig metallische und isolierende Phasen vor, so kann der Metall-IsolatorÜbergang und der damit verbundene CMR-Effekt im Rahmen eines Perkolationsübergangs verstanden werden. Dabei verändert sich in Abhängigkeit von der Temperatur das Verhältnis von metallischer und isolierender Phase. Ab einer kritischen Temperatur bzw.

\footnotetext{
${ }^{4}$ CER steht für colossal electroresistance effect. Im Gegensatz zum CMR wird hier die Widerstandsänderung elektrisch induziert.

${ }^{5}$ Dagotto weist darauf hin, dass der Begriff der Phase im thermodynamischen Sinn nicht gerechtfertigt ist, da hierfür eine große Zahl von Elektronen notwendig ist. 25]
} 
einem kritischen Anteil an metallischer Phase bilden sich Perkolationspfade, wodurch der Widerstand drastisch verringert wird. Mayr et al. modellieren den Perkolationsübergang im Rahmen eines Zufallswiderstandsnetzwerkes. [46] Dieses kann den steilen Metall-IsolatorÜbergang in dem $\mathrm{La}_{1-x-y} \mathrm{Pr}_{y} \mathrm{Ca}_{x} \mathrm{MnO}_{3}$-System erklären und zeigt gute Übereinstimmung mit den experimentellen Daten von Uehara et al. [45].

Im Gegensatz dazu finden Mathieu et al. in $\mathrm{Sm}_{0,5} \mathrm{Ba}_{0,5} \mathrm{MnO}_{3}$-Einkristallen mittels Röntgenbeugung und AC-Suszeptibiltät keine Hinweise für eine elektronische Phasenseparation. 47] Auch Köster et al. finden in temperatur- und magnetfeldabhängigen STM/STSMessungen an $\mathrm{La}_{0,75} \mathrm{Ca}_{0,25} \mathrm{MnO}_{3}$-Schichten keine Anzeichen für perkolierendes Verhalten oder eine elektronische Phasenseparation zwischen $2 \ldots 1000 \mathrm{~nm}$. [48] Weitere STM/STSArbeiten an einem Kation-geordneten und einem Kationen-ungeordneten Film zeigen nur für den Kationen-ungeordneten Film eine elektronische Phasenseparation, obwohl der CMR-Effekt in beiden Schichten ausgeprägt ist. [49]

So können auch die Konzepte der elektronischen Phasenseparation und der Einfluss von polaronischen Korrelationen den CMR-Effekt und die reichhaltigen Phasendiagramme der Manganate bisher noch nicht zufrieden stellend erklären.

\subsection{Phasendiagramm von $\mathrm{La}_{1-\mathrm{x}} \mathrm{Sr}_{\mathrm{x}} \mathrm{MnO}_{3}$}

Auf der Grundlage der bisher beschriebenen Schemata und Modellvorstellungen wird im Folgenden der Blick auf ein konkretes Manganat gerichtet, das $\mathrm{La}_{1-\mathrm{x}} \mathrm{Sr}_{\mathrm{x}} \mathrm{MnO}_{3}$-System. Hierbei handelt es sich um das Manganatsystem, welches in der vorliegenden Arbeit hauptsächlich untersucht wird. Eine Skizze des Phasendiagramms von $\mathrm{La}_{1-\mathrm{x}} \mathrm{Sr}_{\mathrm{x}} \mathrm{MnO}_{3}$ findet sich in den Referenzen [25, 26, 28, 50, 51].

Bei $\mathrm{LaMnO}_{3}(x=0)$ ist das $\mathrm{e}_{g}$-Band mit jeweils einem Elektron pro Manganion gefüllt. Wie bereits beschrieben, führt der Superaustausch-Mechanismus bei $\mathrm{LaMnO}_{3}$ zu einem antiferromagnetischen, isolierenden Grundzustand. [30] Des Weiteren kommt es zu einem kollektiven Jahn-Teller-Effekt, der mit einer orbitalen Ordnung verbunden ist (siehe Abb. 2.6). Jahn-Teller-Ionen sind noch bis Dotierungen von $x=0,175$ vorhanden. Ab dieser Dotierung findet sich ein struktureller Phasenübergang von der orthorhombischen zur rhomboedrischen Perowskitstruktur (siehe Abb. 2.3). Der Grundzustand für $x>0,175$ ist ferromagnetisch und metallisch. $\mathrm{La}_{1-\mathrm{x}} \mathrm{Sr}_{\mathrm{x}} \mathrm{MnO}_{3}$ zeigt für $x>0,175$ eine (für Manganate) sehr große Bandbreite und wird als prototypisches Doppelaustauschsystem beschrieben (canonical double exchange system [28]). Das liegt an der vergleichsweise geringen Elektron-Gitter-Wechselwirkung und Coulomb-Korrelations-Effekten. [28] Bei einer optimalen Dotierung von $x=0,3$ bzw. in $\mathrm{La}_{0,7} \mathrm{Sr}_{0,3} \mathrm{MnO}_{3}$ kann die Curie-Temperatur auf bis zu $T_{\mathrm{C}}=370 \mathrm{~K}$ ansteigen.

In Abhängigkeit von der Dotierung findet sich für Temperaturen oberhalb der Curie-Temperatur bei $x=0,26$ ein Übergang von isolierendem zu metallischem Verhalten. In anderen Worten: Als Funktion der Temperatur findet für $x>0,26$ kein Metall-Isolator-Übergang, der mit einem Vorzeichenwechsel von $d \rho / d T$ verbunden ist, mehr statt. Hier ist in dem $\rho(T)$-Verlauf bei der Curie-Temperatur lediglich ein Knick zu sehen. [50] Für größere Werte von $x$ (ab etwa $x>0,5$ ) ergibt sich ein wiederum antiferromagnetischer, isolierender Grundzustand. [25]

In den Manganaten sind viele verschiedene Ordnungszustände energetisch ähnlich, daher reichen oft kleine Variationen verschiedenster Parameter aus, um zu neuen oder veränderten strukturellen, elektronischen oder magnetischen Ordnungen zu gelangen. Manganatproben können nominell die gleiche Dotierung haben, aber dennoch sehr unterschiedliche 
Curie-Temperaturen zeigen. 52 Mitchell et al. erstellten sogar ein Phasendiagramm für $\mathrm{La}_{1-\mathrm{x}} \mathrm{Sr}_{\mathrm{x}} \mathrm{MnO}_{3}(0<x<0,25)$ nicht nur in Abhängigkeit der Temperatur, sondern auch als Funktion der Konzentration von Sauerstoffleerstellen. [29] Auch die Oberflächen von Manganatproben oder auch Manganatgrenzflächen zeigen aufgrund von Symmetriebrechung und veränderten Spannungszuständen andere magnetische, elektronische und strukturelle Phasen. 


\section{Elektrisches Widerstandsschalten}

Zur nichtflüchtigen Datenspeicherung ist heutzutage die Silizium basierte Flash-Technologie weit verbreitet. Schwachstellen der Flash-Technologie sind begrenzte Lebensdauern, langsame Schreibgeschwindigkeiten und hohe Spannungen, die zum Schreiben der Daten benötigt werden. Zusätzlich kommt die fortschreitende Miniaturisierung an ihre physikalischen Grenzen. [53] Deswegen ist das Interesse an neuartigen Konzepten zur nichtflüchtigen Datenspeicherung enorm. Kandidaten, die zur Debatte stehen, sind (unter anderen) Systeme beruhend auf dem phase change memory-Konzept, eines elektrochemischen Metallisierungs-Prozesses oder dem elektrischen Widerstandsschalten in Übergangsmetalloxiden. [54] Alle diese Systeme zeigen remanente, reversible Widerstandsänderungen in Abhängigkeit von der Vorgeschichte des elektrischen Signals. [53, 54] Der Widerstand kann zwischen zwei (oder mehr) Widerstandswerten eingestellt werden, wobei die unterschiedlichen Widerstandsbeträge als Bits zur Datenspeicherung verwendet werden können. Die Strukturen sind dabei typischerweise wie ein Kondensator aufgebaut. Hierbei sind zwei metallische Elektroden durch eine isolierende oder halbleitende Barriere voneinander getrennt. In diesem Fall wird von einer Metall-Isolator-Metall-Struktur gesprochen (MIMStruktur), hiervon ist in Abb. 3.1 ein Schema gezeigt.

Eine prominentes Beispiel für eine MIM-Struktur (mit reversiblen und remanenten Widerstandsänderungen in Abhängigkeit von der elektrischen Vorgeschichte) beruht auf dem phase change memory-Konzept: Zwischen zwei metallischen Elektroden befindet sich das den Widerstand verändernde Material. Typische phase change memory-Materialien sind Chalkogenide wie $\mathrm{Ge}_{2} \mathrm{Sb}_{2} \mathrm{Te}_{5}$. [54] Die unterschiedlichen Widerstandszustände beruhen auf der amorphen und kristallinen Phase des Chalkogenids. Die amorphe Phase wird eingestellt, indem ein hoher Strompuls für eine sehr kurze Zeit angelegt wird, hierbei schmilzt das Material und bleibt aufgrund der schnellen Abkühlrate im amorphen Zustand. Die niederohmigere, kristalline Phase wird durch einen länger anhaltenden Strompuls mit niedrigerem Stromfluss eingestellt.

Ein weiteres Beispiel für eine Widerstandsänderung durch ein elektrisches Signal beruht auf einem elektrochemischen Metallisierungs-Prozess. In diesem Fall bildet sich von einer Elektrode ein Pfad aus Elektrodenmaterial in das sich zwischen den Elektroden befindende, isolierende Material. Hierfür sind zwei verschiedene Elektrodenmaterialien notwendig, zum einen eine „aktive“ Elektrode (etwa aus Kupfer oder Silber) und eine chemisch inerte 


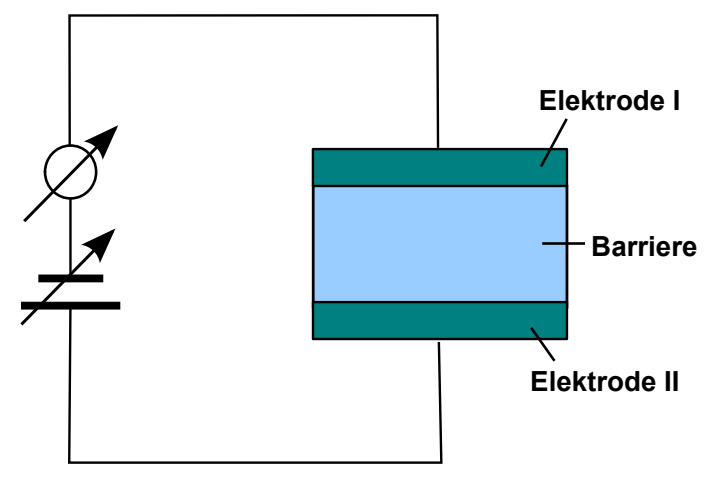

(a) Sandwich-Struktur

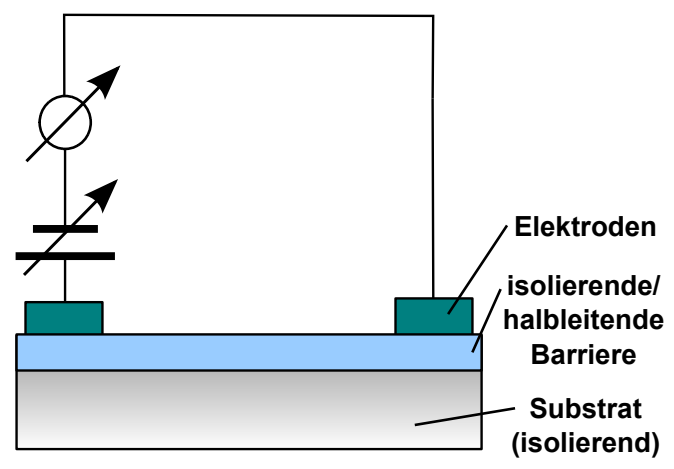

(b) planarer Aufbau

Abb. 3.1: Typische Realisierungen von MIM-Strukturen.

Elektrode. Unter dem Einfluss eines elektrischen Feldes werden die Ionen von der aktiven Elektrode oxidiert und wandern durch das Ionen leitende, die Elektroden trennende Material. An der chemisch inerten Elektrode kommt es zur Reduktion der Ionen, wodurch sich metallische Dendriten in dem Zwischenmaterial bilden. Wenn die beiden Elektroden durch einen metallischen Pfad kurzgeschlossen sind, ist die Zelle im niederohmigen Zustand. Wird die Polarität des elektrischen Feldes umgekehrt, so wird das metallische Filament wieder aufgelöst; die Zelle schaltet in den hochohmigen Widerstandszustand. [17]

Zu einer dritten Klasse, die reversible und remanente Widerstandsänderungen in Abhängigkeit der Vorgeschichte des elektrischen Signals zeigen, werden die Übergangsmetalloxide gezählt. Hierbei befindet sich zwischen den metallischen Elektroden ein einfaches Übergangsmetalloxid wie etwa $\mathrm{NiO}$ [55, 56] oder auch ein komplizierteres Mehrkomponentensystem wie das perowskitische Manganat [9, 14]. In diesem Kapitel bzw. in dieser Arbeit wird der Fokus auf das resistive Widerstandsschalten der Übergangsmetalloxide mit Perowskitstruktur gelegt. Bei dieser Materialklasse wird der zugrunde liegende, physikalische Mechanismus kontrovers diskutiert, die am weitesten verbreiteten Modellvorstellungen werden im Abschnitt 3.2 vorgestellt. Zunächst folgt jedoch eine kurze Einführung in mögliche Klassifikationen der Schaltprozesse.

Als grundlegende und weiterführende Literatur sind besonders die Veröffentlichungen von Sawa [16] und von Waser und Auno [17] zu nennen.

\subsection{Klassifizierung des Widerstandsschaltens}

Der Effekt des elektrischen Widerstandsschaltens kann anhand von unterschiedlichen StromSpannungs-Charakteristiken eingeteilt werden. Eine weitere Einteilung kann aufgrund der räumlichen Lage des schaltenden Bereichs in den Elementen vorgenommen werden. Diese verschiedenen Klassifizierungsmöglichkeiten werden im Folgenden vorgestellt.

\section{Strom-Spannungs-Kennlinien}

Als erstes wird das Widerstandsschalten aufgrund von unterschiedlichen Strom-SpannungsKennlinien charakterisiert. Ein Schema für die idealisierten Schaltcharakteristiken dieser 


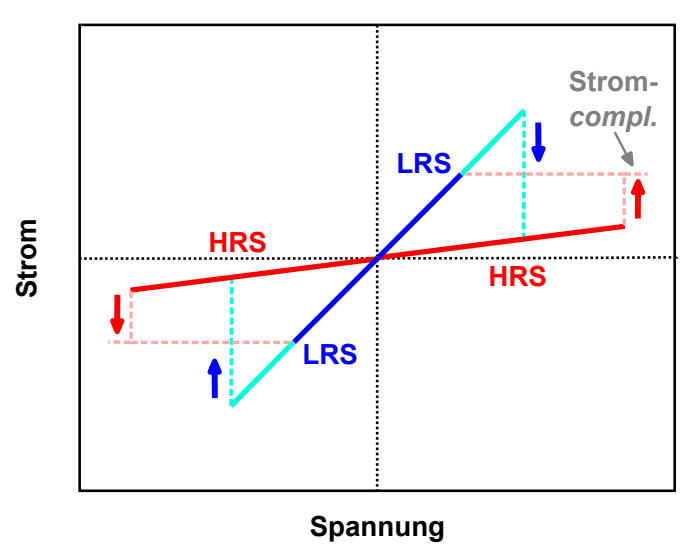

(a) unipolar

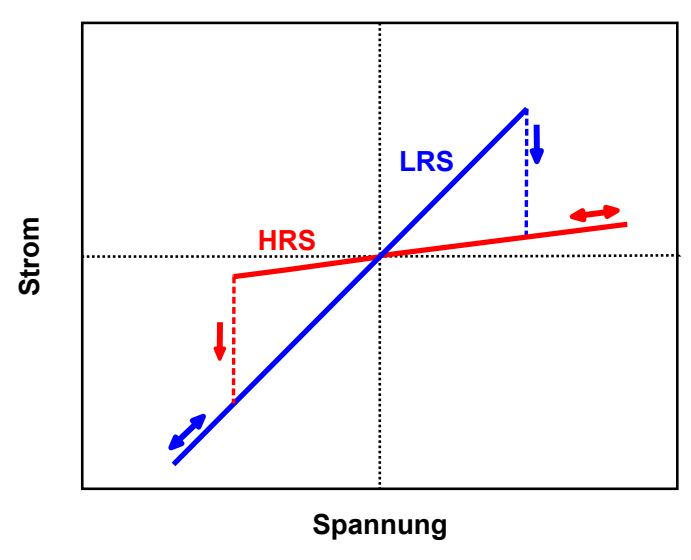

(b) bipolar

Abb. 3.2: Unipolare und bipolare Strom-Spannungs-Schaltcharakteristiken nach Waser und Auno [17].

Unterteilung findet sich in Abb. 3.2: Es wird zwischen unipolarem und bipolarem Widerstandsschalten unterschieden.

Bei einem unipolaren (oder symmetrischen) Schaltvorgang spielt die Polarität des elektrischen Signals (Strom oder Spannung) keine Rolle. Die verschiedenen Widerstandswerte werden eingestellt, in dem unterschiedlich große Strom- oder Spannungswerte überschritten werden. Befindet sich der Widerstand in einem hochohmigen Zustand (HRS: high resistive state), so wird der tiefohmige Zustand (LRS: low resistive state) durch das Überschreiten einer kritischen Spannung bzw. eines kritisches Stroms erreicht. Dabei wird der maximale Stromfluss über einen Strom-compliance kontrolliert. Der LRS bleibt solange erhalten, bis wieder der für den tiefohmigen Zustand kritische Schwellwert überschritten wird. Üblicherweise zeigen die einfachen, binären Metalloxide das unipolare Widerstandsschalten. Auch die phase change memory-Strukturen haben prinzipiell eine unipolare Schaltcharakteristik, allerdings ist hier kein Strom-compliance notwendig. [17]

Im Gegensatz dazu spielt bei dem bipolaren Widerstandsschalten (asymmetrischer Schaltprozess) die Polarität die entscheidende Rolle. HRS und LRS werden mit (genügend großen) Spannungspulsen unterschiedlicher Polarität hin und her geschaltet. Die bipolare Schaltcharakteristik wird meist in den komplizierten Übergangsmetalloxiden gefunden, wie etwa $\mathrm{Pr}_{0,7} \mathrm{Ca}_{0,3} \mathrm{MnO}_{3}$ [14], $\mathrm{SrTiO}_{3}$ [18, 19] und $\mathrm{SrZrO}_{3}$ [20]. Es ist zu beachten, dass für bipolares Widerstandsschalten eine Asymmetrie im System vorhanden sein muss (zum Beispiel unterschiedliche Elektrodenmaterialen). [17]

\section{Räumliche Verteilung der schaltenden Bereiche}

Für manche Materialien kann eine Klassifizierung aufgrund der lokalen Lage der den Widerstand schaltenden Bereiche in den Elementen vorgenommen werden. Dabei kann zwischen dem Fall, in dem der schaltende Bereich an der Grenzfläche zwischen der Elektrode und dem Oxid lokalisiert ist, und der Ausbildung eines leitfähigen Filaments, das die Elektroden miteinander verbindet, unterschieden werden. Bei einem filamentären Schaltprozess resultieren der hoch- und tiefohmige Zustand daraus, dass das Filament während des Schaltprozesses unterbrochen bzw. wieder geschlossen wird. 
Ein Beispiel für einen filamentartigen Prozess wird von Fujiwara et al. vorgestellt. [57] Dazu wurde eine planare Anordnung einer MIM-Struktur aus Metal/CuO/Metall im hochund im tiefohmigen Zustand in einem Rasterelektronenmikroskop untersucht. Das $\mathrm{CuO}$ zeigt das für binäre Metalloxide üblichere, unipolare Schalten, wozu aber zu Beginn ein Elektroformierungsschritt (electroforming) notwendig ist. Während der Elektroformierung wird eine hohe Spannung (weit höher als für den Schaltvorgang notwendig) an die Struktur gelegt, bis diese in den tiefohmigen Zustand schaltet. Die RasterelektronenmikroskopAufnahmen zeigen nach dem Elektroformierungsschritt ein Filament, welches die beiden Elektroden miteinander verbindet. Wird dieses Filament mittels eines fokussierten Ionenstrahls (FIB) durchgeschnitten, so ist die Struktur wieder im HRS. Auch im Bereich der perowskitischen Übergangsmetalloxide wird filamentartiges Schalten beobachtet. Szot et al. untersuchten $\mathrm{SrTiO}_{3}$-Einkristalle, die bipolare Widerstandscharakteristiken zeigen. [19] In den Kristallen finden sie eine filamentartige Leitfähigkeit entlang von Pfaden mit Durchmessern von etwa $2 \ldots 4 \mathrm{~nm}$, wobei die Pfade mit Versetzungen identifiziert werden konnten.

Ein weiteres Experiment zur Analyse der geometrischen Verteilung der schaltenden Bereiche beinhaltet die Untersuchung der Abhängigkeit der Widerstände im HRS und LRS von der Größe der Kontaktpads. Sim et al. charakterisierten diese Abhängigkeit sowohl für $\mathrm{Nb}: \mathrm{SrTiO}_{3}$ - als auch $\mathrm{NiO}_{x}$-Proben. Nur für die $\mathrm{Nb}: \mathrm{SrTiO}_{3}$-Proben wird eine Abhängigkeit von der Größe der Kontaktpads beobachtet. Dieses wird als Indiz für eine uniforme Verteilung des schaltenden Bereichs gewertet. [58]

Experimentelle Hinweise auf eine Lokalisierung des Widerstandsschaltens auf den Bereich der Grenzfläche zwischen dem Zwischenmaterial und den Elektroden finden sich für das Manganatsystem $\operatorname{Pr}_{0,7} \mathrm{Ca}_{0,3} \mathrm{MnO}_{3}$ : Baikalov et al. untersuchten bipolares Widerstandsschalten in $\mathrm{Ag} / \mathrm{Pr}_{0,7} \mathrm{Ca}_{0,3} \mathrm{MnO}_{3} / \mathrm{YBa}_{2} \mathrm{Cu}_{3} \mathrm{O}_{7}$-Strukturen. [15] Sie finden mittels Widerstandsmessungen an einer Vielzahl von Kontaktpads, dass das Widerstandsschalten an der Kontaktfläche von $\mathrm{Ag} / \mathrm{Pr}_{0,7} \mathrm{Ca}_{0,3} \mathrm{MnO}_{3}$ lokalisiert ist. Allerdings zeigen Ignatiev et al. ebenfalls in $\mathrm{Pr}_{0,7} \mathrm{Ca}_{0,3} \mathrm{MnO}_{3}$ mittels scanning Kelvin probe microscopy, dass zwar das Widerstandsschalten hauptsächlich an der Grenzfläche stattfindet, allerdings auch einen kleiner Anteil an Widerstandsschalten im Volumen (bulk) der Probe zu finden ist. [59]

Es ist daher nicht einfach oder auch in allen Fällen sinnvoll, eine stringente Einteilung in filamentäres und Grenzflächenwiderstandsschalten bzw. eine Einteilung zwischen Volumen oder Grenzfläche vorzunehmen. Zusammenfassend lässt sich allerdings für die Manganatsysteme die Aussage treffen, dass eher kein filamentärer Prozess beobachtet wird. [60]

\subsection{Mikroskopische Modellvorstellungen}

In den vorherigen Abschnitten wurde eine allgemeine Klassifizierung des Widerstandsschaltens vorgestellt. Im Folgenden wird eine Einführung in die am weitesten verbreiteten, mikroskopischen Modellvorstellungen für das bipolare Widerstandsschalten gegeben. Es ist anzumerken, dass auch innerhalb der Materialklasse der Perowskite bei ähnlichen Schaltcharakteristiken der zugrunde liegende, mikroskopische Mechanismus unterschiedlich sein kann. [61] Daher wird in dieser Arbeit der Schwerpunkt auf Ergebnisse bzw. Modellvorstellungen für die Manganatsysteme gelegt.

\subsubsection{Schottky-Barriere}

Wie eingangs beschrieben ist ein Schaltelement aus zwei metallischen Elektroden, zwischen denen sich ein isolierendes oder halbleitendes Oxid befindet, aufgebaut. An der 
Grenzfläche zwischen dem Oxid und dem Metall kann daher ein Schottky-Kontakt vorliegen. Wenn die Schottky-Barriere durch ein elektrisches Signal verändert werden kann, resultieren hieraus verschiedene Widerstandswerte. In anderen Worten: Das Widerstandsschalten kann über eine Veränderung der Schottky-Barriere durch ein elektrisches Signal erklärt werden. Bei dieser mikroskopischen Modellvorstellung wird angenommen, dass das Widerstandsschalten im Bereich der Grenzfläche lokalisiert ist.

Sawa et al. schlagen vor, dass die Schottky-Barriere über Ladungsträger, die in Grenzflächenzuständen gefangen werden, verändert werden kann. 62] Zu dieser Modellvorstellungen gelangten sie durch die Untersuchung von Sandwich-Strukturen, die aus $\mathrm{SrRuO}_{3}$ - und $\mathrm{Pr}_{0,7} \mathrm{Ca}_{0,3} \mathrm{MnO}_{3}$-Schichten bestehen $\left(\mathrm{SrRuO}_{3}\right.$ diente dabei als untere Elektrode). Bei der Verwendung von verschiedenen (oberen) Elektrodenmaterialen beobachteten Sawa et al. unterschiedliches Schaltverhalten, welches durch eine veränderte Schottky-Barriere erklärt wird. ${ }^{1}$ In den Proben, die Widerstandsschalten zeigen, wird das Schaltverhalten dabei wie folgt erklärt: Liegt eine genügend große Spannung an der Grenzfläche kann eine große Anzahl von Elektronen in Grenzflächenzustände gebracht bzw. extrahiert werden. Durch die veränderte Ladung kommt es zu einer Veränderung der Höhe bzw. der Breite von der Schottky-Barriere, woraus die unterschiedlichen Widerstandswerte resultieren. In einer weiteren Arbeit (von denselben Autoren) wird nicht das Elektrodenmaterial, sondern die Manganatschicht verändert. 63 Diese besteht aus einer Kombination von $\mathrm{La}_{0,7} \mathrm{Sr}_{0,3} \mathrm{MnO}_{3}$ (LSMO) und $\mathrm{Sm}_{0,7} \mathrm{Ca}_{0,3} \mathrm{MnO}_{3}$ (SCMO), wobei die Dicke der SCMO-Schicht variiert wird. Bei einer reinen LSMO-Schicht wird kein Widerstandsschalten beobachtet. Wird zwischen die LSMO-Schicht und die Titan-Elektrode eine Einheitszellen-dicke SCMO-Schicht eingebracht, so wird bipolares Widerstandsschalten gefunden. ${ }^{2}$ Daraus wird geschlossen, dass die für den Schaltprozess aktive Lage einige Einheitszellen dick und das Widerstandsschalten in einer elektronischen Ursache begründet ist.

\subsubsection{Sauerstoffleerstellen-Diffusion und Elektromigration}

Das am weitesten verbreitete Modell zur Erklärung des Widerstandsschaltens basiert auf einer Sauerstoffleerstellen-Diffusion oder Elektromigration. Eine erste Arbeit im Bereich der Manganate wurde von Baikalov et al. vorgestellt. [15] Sie untersuchten $\operatorname{Pr}_{0,7} \mathrm{Ca}_{0,3} \mathrm{MnO}_{3^{-}}$ Schichten (unter anderem) in planarer Geometrie mit Silber-Kontakten. Das beobachtete, bipolare Widerstandsschalten wird mit der Entstehung bzw. der Diffusion von Sauerstoffleerstellen im elektrischen Feld erklärt, wobei als Indiz hierfür die langsame Dynamik des Schaltprozess genannt wird.

Quintero et al. untersuchten die Temperaturabhängigkeit des Schalteffektes in (La, Pr, Ca)Manganat Polykristallen $(y=0,3$ und 0,32$)$ und fanden den größten Schalteffekt bei einer Temperatur $T^{*}$ unterhalb der Curie-Temperatur. [64] Um die Ergebnisse zu erklären,

\footnotetext{
${ }^{1}$ Die verwendeten oberen Elektrodenmaterialen waren Titan, $\mathrm{SrRuO}_{3}$, Platin, Silber und Gold; nur für die Titan-Elektrode wurde Widerstandsschalten beobachtet. Silber und Titan besitzen ähnliche Austrittsarbeiten. Der Unterschied zwischen diesen Elektrodenmaterialen wird durch die große Sauerstoffaffinität des Titans begründet. Bei der Verwendung einer Titan-Elektrode könnte diese den Sauerstoff aus dem $\mathrm{Pr}_{0,7} \mathrm{Ca}_{0,3} \mathrm{MnO}_{3}$ (PCMO) extrahieren, woraus eine höhere Dichte an Grenzflächenzuständen gegeben sein kann. Dieses kann zu einer größeren Bandverbiegung an der Grenzfläche zwischen dem Ti/PCMO als bei Ag/PCMO führen. 62]

${ }^{2}$ Die Schalteffekte (Schaltamplitude) werden mit steigender Dicke der SCMO-Schichten größer und der Schaltprozess wird schneller. Ist die SCMO-Schicht lediglich eine Einheitszelle dick, so muss die Spannung für $\tau=10 \mu \mathrm{s}$ lang anliegen, bei einer Dicke von fünf Einheitszellen dagegen reichen $\tau=100 \mathrm{~ns}$ aus. 63
} 
nehmen sie an, dass die Spannungspulse die lokale Sauerstoffleerstellen-Konzentration verändern. Über diese Veränderung können die elektronischen Zustände an der Grenzfläche dotiert und damit der Widerstand geschaltet werden.

Tsui et al. untersuchten mittels Impedanz-Spektroskopie die Schalteffekte in Schichten aus $\operatorname{Pr}_{0,7} \mathrm{Ca}_{0,3} \mathrm{MnO}_{3}$ und beobachten, dass der Schaltprozess mit einer kleinen Veränderung der Kapazität verbunden ist. [65] Mit weiteren temperaturabhängigen Messungen des Widerstandes und der I(V)-Kennlinien fanden sie, dass es eine isolierende Schicht (dünner als $10 \mathrm{~nm}$ ) zwischen den Elektroden und dem $\operatorname{Pr}_{0,7} \mathrm{Ca}_{0,3} \mathrm{MnO}_{3}$ gibt. Die Diskussion beruht darauf, dass das elektrische Feld kristalline Defekte erzeugt, so dass der elektronische Transport über einen space charge limited current dominiert wird. Die Ladungsträger werden durch das elektrische Feld der schon im Material vorhandenen Ladungsträger blockiert. Durch die Spannungspulse wird die Anzahl der Defekte verändert, womit das Widerstandsschalten erklärt wird.

Eine weiteres Modell, dass auf der elektrochemischen Migration von Sauerstoffleerstellen in der Nähe der Grenzflächen beruht, erklärt die unterschiedlichen Widerstände über eine Veränderung der Breite einer Schottky-Barriere. [16] Damit gibt es auch bei der Annahme einer Sauerstoffleerstellen-Diffusion oder Elektromigration im elektrischen Feld unterschiedliche Ansätze zur Erklärung der verschiedenen Widerstände. Als letztes soll noch auf die Bedeutung von höherdimensionalen Defekten - im Rahmen der SauerstoffleerstellenDiffusion - hingewiesen werden. Szot et al. schlagen aufgrund ihrer Arbeiten an $\mathrm{SrTiO}_{3}$ vor, dass ein erhöhter Massentransport entlang von Versetzungen stattfinden kann (Stichwort: pipe diffusion). [19]

\subsubsection{Struktureller Übergang}

Die starke Kopplung der elektronischen und strukturellen Freiheitsgrade in den Manganaten führt zu der Fragestellung, welcher Zusammenhang zwischen der kristallographischen Struktur und den verschiedenen Widerstandswerten existiert. Um diesen Zusammenhang zu untersuchen, sind Messungen der lokalen Struktur im elektrischen Feld von besonderer Bedeutung. Hierzu zählen Transmissionselektronenmikroskop-Messungen (TEM), in denen das Widerstandsschalten lokal durch eine STM-Spitze induziert werden kann. Eine solche Untersuchung des Widerstandsschaltens im TEM wurde von Jooss et al. an $\operatorname{Pr}_{0,7} \mathrm{Ca}_{0,3} \mathrm{MnO}_{3}$-Schichten durchgeführt. [36, 61] Im elektrischen Feld werden strukturelle Modifikationen bzw. die kollektive Verkippung und Verdrehung der $\mathrm{MnO}_{6}$-Oktaeder beobachtet. Dieses wird im Rahmen eines Übergangs der polaronischen Ordnung erklärt, die mit einer Bandverbiegung in der Nähe der Grenzfläche verbunden ist.

In der Arbeit von Krisponeit [60] wird das Widerstandsschalten mittels C-AFM Messungen (C-AFM: conductive-AFM, siehe Kap. 4.7) untersucht und im Rahmen eines strukturellen Übergangs an der Manganatoberfläche erklärt. Ein Funktionsschema dieses Modells ist in Abb. 3.3 gezeigt. Im HRS sind die Jahn-Teller verzerrten Orbitale innerhalb der Probenoberfläche ausgerichtet. Wird zwischen der leitfähigen Spitze des AFM-Cantilevers und der Manganatprobe eine immer größer werdende, positive Spannung (die Probe ist geerdet) angelegt, kann die elektrostatische Energie des in der Ebene liegenden Jahn-Teller-Oktaeders im Vergleich zu dem zweiten $\mathrm{e}_{g}$-Orbital des Manganions erhöht werden; dadurch kann die Entartung der Jahn-Teller aufgespalteten Zustände wiederhergestellt werden. Dieses entspricht einem Übergang zu einer Jahn-Teller unverzerrten Struktur, welche typischerweise eine höhere Leitfähigkeit hat.

Der leitfähigere Bereich kann sich innerhalb der Manganatprobe durch den elektrischen Feldgradienten an der Phasengrenze ausdehnen und die Jahn-Teller-Verzerrung des hoch- 


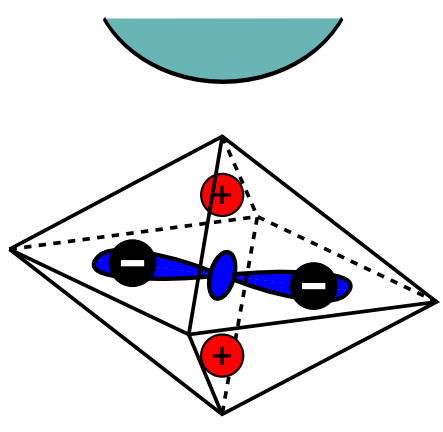

(a) HRS, Jahn-Teller verzerrt

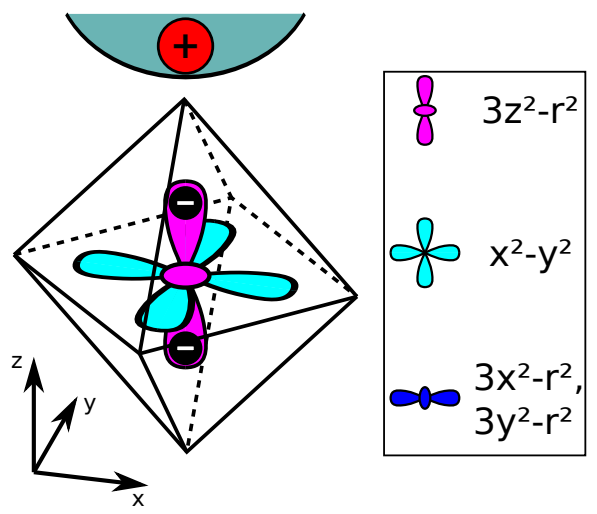

(b) LRS

Abb. 3.3: Schema zur Erklärung eines strukturellen Übergangs im elektrischen Feld. Mit freundlicher Genehmigung von Krisponeit. 60]

ohmigen Bereichs weiter aufheben. Eine Remanenz des tiefohmigen Zustands kann erreicht werden, wenn die Volumenenergie der Grenzflächenenergie entspricht, wobei auch PinningEffekte eine Rolle spielen.

\subsection{Technologische Bedeutung}

Das Widerstandsschalten ist im Hinblick auf eine mögliche technologische Anwendung als nichtflüchtiges Speichermedium bzw. RRAM (resistive random access memory) interessant. Eine prägnante Übersicht über die für die Anwendung als nichtflüchtiges Speichermedium relevanten Parameter, etwa in welchen Bereichen die Schaltspannungen liegen müssen oder wie das Widerstandsverhältnis zwischen HRS und LRS sein muss, findet sich in der Zusammenfassung von Waser et al. (Ref. [53]). In diesem Zusammenhang ist es erwähnenswert, dass bereits im Jahr 2002 ein erster RRAM-Prototyp von Zhuang et al. vorgestellt wurde. Es wurde eine 64 bit RRAM-Zelle demonstriert, in der das aktive Material zwischen den Elektroden aus $\operatorname{Pr}_{0,7} \mathrm{Ca}_{0,3} \mathrm{MnO}_{3}$ besteht. 66]

Neben der potentiellen Anwendungsmöglichkeit als RRAM wird von Strukov et al. darauf hingewiesen, dass das bipolare Widerstandsschalten dem Verhalten eines Memristors bzw. dem eines memristiven Systems entspricht. [67] Ein Memristor ist eines von vier fundamentalen, passiven Bauelementen, die von Chua [68] aufgrund von Symmetrieargumenten theoretisch vorhergesagt wurden. Die bekannten, passiven elektrischen Bauelemente sind der Widerstand, die Kapazität und die Induktivität - diese verknüpfen die fundamentalen Größen (Ladung $q$, magnetischer Fluss $\varphi$, Strom $i$ und Spannung $u$ ) miteinander. Das fehlende Element - der Memristor $M$ - verbindet den magnetischen Fluss mit der elektrischen Ladung über den funktionellen Zusammenhang $d \varphi=M d q$. Die grundlegende Definition für einen Memristor ist über

$$
u=\frac{d \varphi}{d t}=M(\omega) i \quad \wedge \quad \frac{d \omega}{d t}=i
$$

gegegeben, wobei die Zustandsvariable $\omega$ hier der elektrischen Ladung $q$ entspricht. Für ein memristives System wird dieser Zusammenhang erweitert, [69] hierbei kann $\omega$ einen Satz von mehreren Zustandsvariablen bezeichnen. Es ist

$$
u=M(\omega, i) i \quad \wedge \quad \frac{d \omega}{d t}=f(\omega, i) .
$$


Als viertes (passives) elektrisches Bauelement ist der Memristor bzw. ein memristives System von fundamentalem Interesse bei der Entwicklung von elektrischen Schaltkreisen, wie bereits von Chua angemerkt wurde. [68, 69]

Schließlich verweisen Ohno et al. auf die Ähnlichkeit zwischen den Widerstandsschaltcharakteristiken und biologischen Synapsen (allerdings in $\mathrm{Ag}_{2} \mathrm{~S}$-Elementen). [70] Mit den $\mathrm{Ag}_{2} \mathrm{~S}$-Elementen kann synaptisches Verhalten nachgestellt werden, daher sind diese für die Entwicklung von künstlichen, neuronalen Netzwerken interessant.

Auf der Grundlage von diesen spannenden, potentiellen Anwendungsmöglichkeiten ist das Gebiet des Widerstandsschaltens ein sehr aktives Forschungsgebiet, mit einer Vielzahl von aktuellen Veröffentlichungen und Konferenzbeiträgen. In dieser Arbeit wird insbesondere der Einfluss und die Bedeutung von ausgedehnten Defektstrukturen auf das Widerstandsschalten untersucht, wozu es - besonders im Bereich der Manganate - bisher nur wenige Veröffentlichungen gibt. 


\section{Experimentelle Techniken}

Das folgende Kapitel dient als Einführung in die in dieser Arbeit verwendeten experimentellen Methoden und Anlagen. Zu Beginn wird im Abschnitt 4.1 die Probenpräparation mittels der Metallorganischen Aerosol Deposition (MAD) beschrieben. Die zur Standardcharakterisierung verwendeten Methoden und Apparaturen, wie die Röntgenstrukturanalyse, Rastersondenmikroskopie, Rasterelektronenmikroskopie und die zu Widerstands- und Magnetisierungsmessungen verwendeten Kryostaten, werden im Anschluss vorgestellt.

Ein Fokus auf die Beschreibung der Details der Schicht-Mikrostrukturierung mittels optischer und Elektronenstrahl-Lithographie wird im Abschnitt 4.4 gelegt. An diesen Mikrostrukturen wird das durch ein elektrisches Feld induzierte Widerstandsschalten untersucht. Weiterhin wird dieses Schalten auf der Nanometer-Skala mittels Leitfähigkeits-AFM Messungen (C-AFM: conductive-AFM) charakterisiert. Details dieser Anlage finden sich im Abschnitt 4.7. Schließlich wird am Ende dieses Kapitels (Abschnitt 4.8) der Aufbau zur Messung von photoinduzierten ersten und dritten harmonischen Spannung eingeführt.

\subsection{Metallorganische Aerosol Deposition}

Die Metallorganische Aerosol Deposition ist ein Verfahren zur Herstellung oxidischer Schichten. Das Funktionsprinzip ist ähnlich der Metallorganischen Gasphasenabscheidung (metal organic chemical vapour deposition). Bei der MAD-Technik wird ein Aerosol aus Metallkomplexen in Lösungsmittel auf ein geheiztes Substrat gesprüht. [71] Zur Herstellung von Manganatschichten ist die Metallorganische Aerosol Deposition besonders gut geeignet, da ein Überschuss an Sauerstoff während der Deposition zur Verfügung gestellt wird. Ein weiterer Vorteil der MAD-Technik ist eine relativ schnelle Probenpräparation, da nicht in einer aufwendigen Vakuumanlage gearbeitet wird. Zusätzlich ist die MAD-Technik sehr flexibel bei der Präparation verschiedener Schichtsysteme und Dotierungen. Im Gegensatz zu der Schichtpräparation mit anderen Methoden (wie etwa der gepulsten Laserdeposition) sind nicht stöchiometrische targets, sondern lediglich die verschiedenen Ausgangspräkursoren notwendig.

Der erste Schritt der Probenpräparation besteht in der Herstellung einer Lösung aus Präkursoren in Dimethylformamid (DMF). Bei den Präkursoren handelt es sich um Acetylacetonatverbindungen der gewünschten Ionen (zum Beispiel La-, Sr- und Mn-Ionen). Die- 


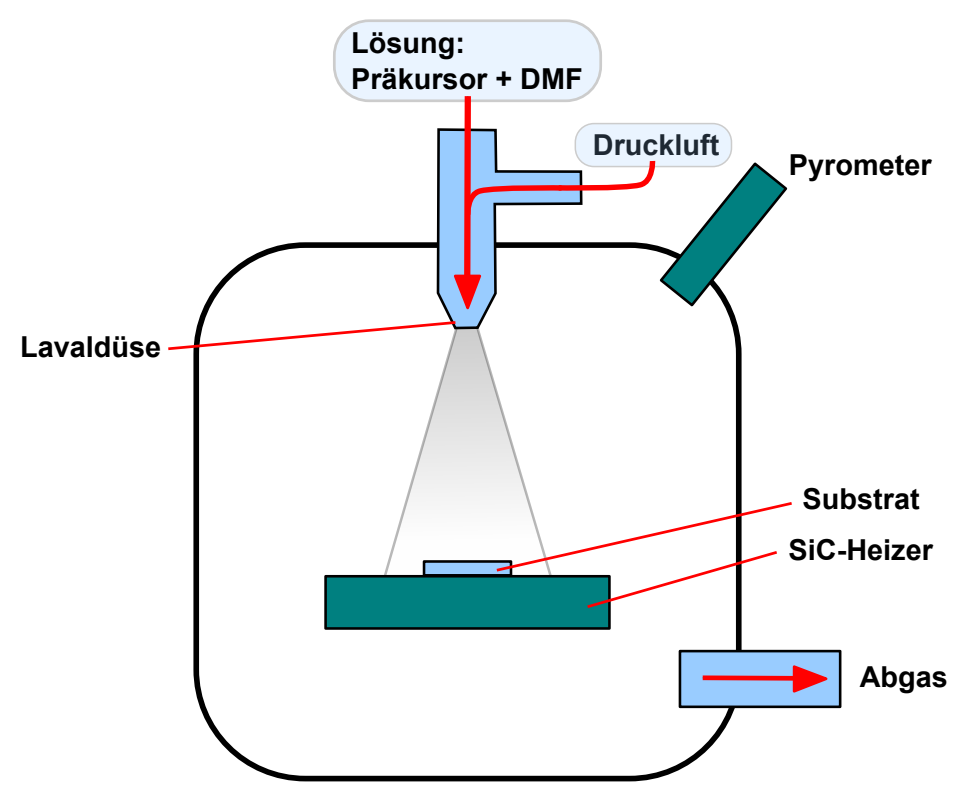

Abb. 4.1: Schema einer MAD-Anlage.

se werden in den geeigneten Mengenverhältnissen in einer Handschuhbox ${ }^{1}$ abgewogen. Die Dotierung einer Schicht kann nur indirekt aus der Masse der abgewogenen Präkursoren bestimmt werden. Um die gewünschte Stöchiometrie zu erhalten, wird eine Probenserie mit unterschiedlichen Präkursoren-Mengenverhältnissen hergestellt. ${ }^{2}$ Die strukturellen, elektronischen und magnetischen Eigenschaften dieser Schichten werden mit bulk-Proben bekannter Dotierung aus der Literatur verglichen. Es muss beachtet werden, dass die Präkursoren sich im Laufe der Zeit verändern können (trotz der Verwendung einer Handschuhbox). Aus diesem Grund muss die Schichtzusammensetzung immer wieder überprüft und optimiert werden.

Nach dem Abwiegen der Präkursoren wird in der MAD-Anlage ein Substrat (z.B. MgO, $\mathrm{SrTiO}_{3}$ oder $\mathrm{Al}_{2} \mathrm{O}_{3}$ ) auf einen SiC-Heizer mittels einer Schablone (ebenfalls aus $\mathrm{SiC}$ ) befestigt. Der Heizer wird zur Schichtdeposition auf $T \approx 980^{\circ} \mathrm{C}$ aufgeheizt, ${ }^{3}$ die Temperatur wird mit einem Pyrometer überprüft. ${ }^{4}$ Die DMF/Präkursor-Lösung wird mittels Druckluft $(p=4 \mathrm{~atm})$ in einer Lavaldüse in ein Aerosol zerstäubt; die mittlere Tröpfengröße ist dabei etwa $20 \mu \mathrm{m}$. [71] Das Aerosol wird auf das geheizte Substrat gesprüht, wobei durch die erhöhte Temperatur oberhalb des Substrates bzw. des Heizers die Pyrolysereaktion beginnt (hier am Beispiel eines Kupfer-Präkursors) [72]

$$
\mathrm{Cu}\left(\mathrm{C}_{3} \mathrm{H}_{5} \mathrm{O}_{2}\right)_{2}+7 \mathrm{O}_{2} \rightarrow \mathrm{CuO}+6 \mathrm{CO}_{2} \uparrow+5 \mathrm{H}_{2} \mathrm{O} \uparrow
$$

\footnotetext{
${ }^{1}$ Die Verwendung einer Handschuhbox ist erforderlich, um die Anreicherung/Verunreinigung der Präkursoren durch Luftfeuchtigkeit zu minimieren.

${ }^{2}$ Auch andere Präparationsparameter wie etwa die Heizertemperatur, die Molarität der Lösung und die Depositionsgeschwindigkeit müssen immer wieder neu optimiert werden.

${ }^{3}$ Die genaue Temperatur auf der Substratoberfläche kann nicht angegeben werden, da die Temperatur mit einem Pyrometer gemessen wird und durchsichtige Substrate verwendet werden.

${ }^{4}$ Bei der Verwendung von MgO-Substraten werden - zur Reinigung des Substrates - vor der Schichtdeposition $3 \mathrm{ml}$ Dimethylformamid (ohne Metallpräkursoren) auf das geheizte Substrat $\left(T \approx 1000^{\circ} \mathrm{C}\right)$ gesprüht.
} 
Unterhalb des Heizers befindet sich ein Ventilator, der für geeignete Strömungsverhältnisse sorgt und die überschüssige Lösung ableitet. Die Schichtdicke wird durch die Molarität und deponierte Menge der DMF/Präkursor-Lösung bestimmt.

Durch eine genaue Regelung der verwendeten Lösungsmenge ist sogar die Deposition von Monolagen möglich. Werden verschiedene Lösungen verwendet, können Multilagen der unterschiedlichen Oxide wie $\mathrm{La}_{1-x} \mathrm{Ca}_{x} \mathrm{MnO}_{3}-$, $\mathrm{LaMnO}_{3}-$ und $\mathrm{BaTiO}_{3}-\mathrm{Multilagen} \mathrm{hergestellt}$ werden. Dieses ermöglicht eine Kombination der physikalischen Eigenschaften der einzelnen Schichten, um neue funktionelle Proben zu erhalten. [73, 74]

\subsection{Röntgenstrukturanalyse}

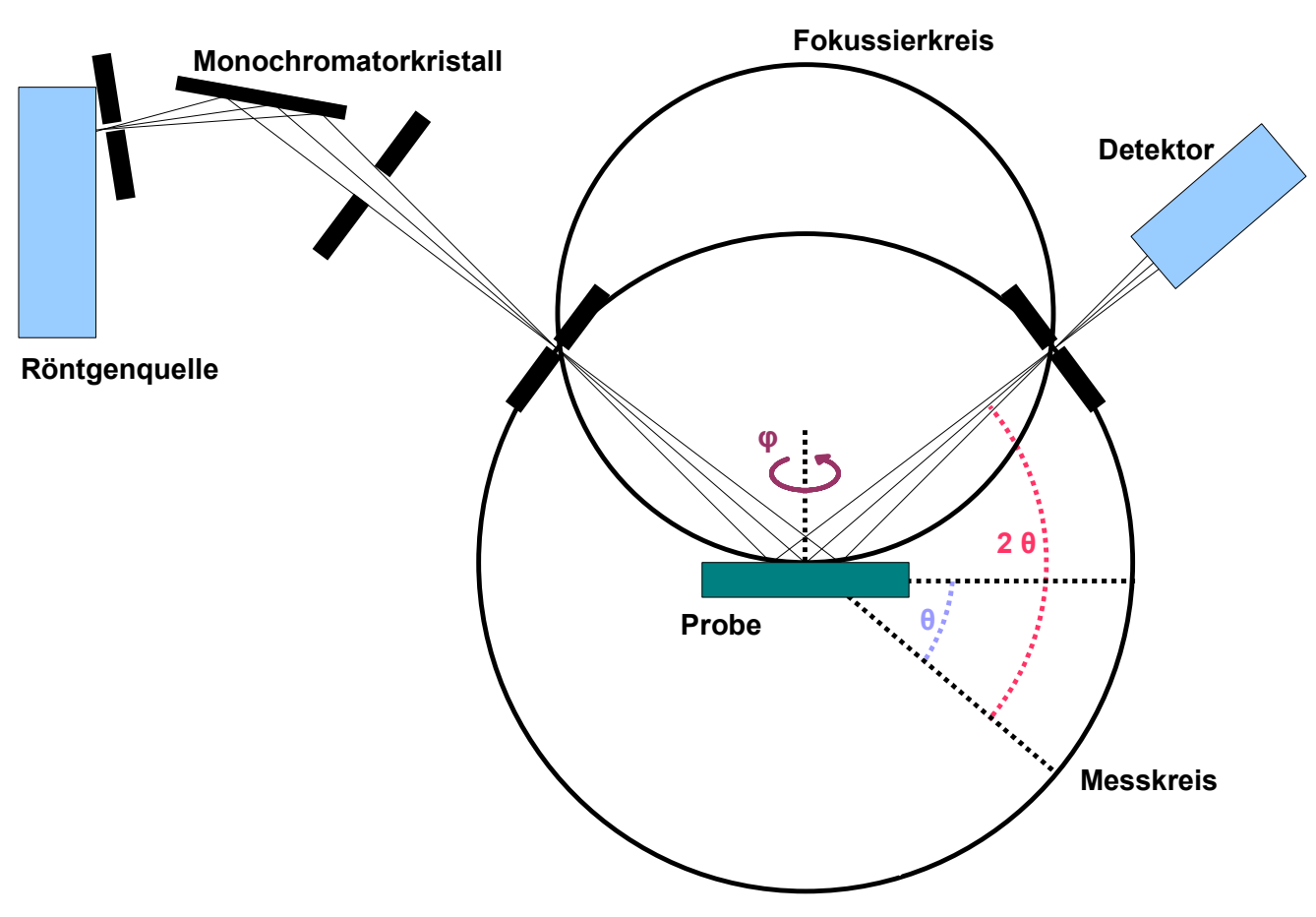

Abb. 4.2: Schematischer Aufbau der verwendeten Röntgenanlagen nach Ref. [75].

Mittels der Röntgendiffraktometrie und -reflektometrie werden strukturelle Eigenschaften wie die Kristallstruktur und -textur sowie Schichtdicken und -rauigkeiten, analysiert. Hierbei wird - im Gegensatz zu den lokalen Methoden wie der Rasterelektronen-, Rasterkraftund Rastertunnelmikroskopie - über ein großes Probenvolumen gemittelt. In dieser Arbeit werden zwei kommerzielle Röntgenanlagen verwendet, zum einen die D8 der Firma Bruker AXS und die D5000 von Siemens. Als Quelle dient in beiden Röntgenanlagen eine Kupferanode, deren $\mathrm{K}_{\alpha}$-Wellenlängen genutzt werden. Die Wellenlänge ergibt sich als gewichtete Mittelung der $\mathrm{K}_{\alpha 1^{-}}$und $\mathrm{K}_{\alpha 2}$-Linie zu $\lambda=1,54184 \AA$.

In Abhängigkeit des betrachteten Winkelbereiches wird zwischen der Kleinwinkelröntgenreflektometrie und der Weitwinkelröntgenbeugung unterschieden. Aus den Weitwinkelmessungen können Kristallstrukturen und -orientierungen, Gitterkonstanten, lokale Verspannungen und Korngrößen bestimmt werden. Hierzu wird die Röntgenstrahlung, wie in Abb. 4.2 gezeigt, in der $\theta$-2 $\theta$-Geometrie in Abhängigkeit des Winkels $\theta$ auf die Probe geleitet; unter dem Winkel $2 \theta$ wird die gebeugte Strahlung detektiert. Intensitätsmaxima entstehen durch konstruktive Interferenz der an verschiedenen Netzebenen gebeug- 
ten Röntgenstrahlung, wie durch die Braggsche Reflexionsbedingung beschrieben wird ( $n \lambda=2 d \sin \theta ; n$ ist eine natürliche Zahl und $d$ der Abstand der Netzebenen). Bei Kenntnis der Kristallstruktur wird aus der Lage der Intensitätsmaxima die Gitterkonstante bestimmt. Die Form der Peaks resultiert aus den Korngrößen ${ }^{5}$, lokalen mikroskopischen Verspannungen und instrumentellen Begebenheiten. [76]

Mittels der Kleinwinkelröntgenreflektometrie werden Schichtdicken und Schichtrauigkeiten bestimmt. Hierzu wird die spiegelnde Reflexion der Röntgenstrahlung an Grenzflächen mit unterschiedlichen Brechungsindizes genutzt. Bei einer dünnen Schicht kommt es zur Reflexion an den Grenzflächen Luft/Schicht und Schicht/Substrat. Die an den verschiedenen Grenzflächen reflektierten Strahlen überlagern sich in Abhängigkeit der Schichtdicke $d$ und des einfallenden Winkels $\theta$. Bei konstruktiver Interferenz ergeben sich Intensitätsmaxima bei den Winkeln $\theta_{i}$

$$
\theta_{i}^{2}=2 \delta+\left(m_{i}+\Delta m\right)^{2} \cdot \frac{\lambda^{2}}{4 d^{2}}
$$

Hierbei entspricht $\delta$ der Dispersion und $m_{i}$ ist eine ganze Zahl, welche die Ordnung des Maximums angibt. Tritt an der Grenzfläche zwischen Schicht und Substrat ein Phasensprung von $\lambda / 2$ auf, so ist $\Delta m=1 / 2$; ansonsten gilt $\Delta m=0$. [77, 78] Die Schichtdicke wird mit dieser Relation nach Hink und Petzold über die Minimierung der Fehlerquadrate bestimmt. [79]

\subsection{Rasterelektronenmikroskopie}

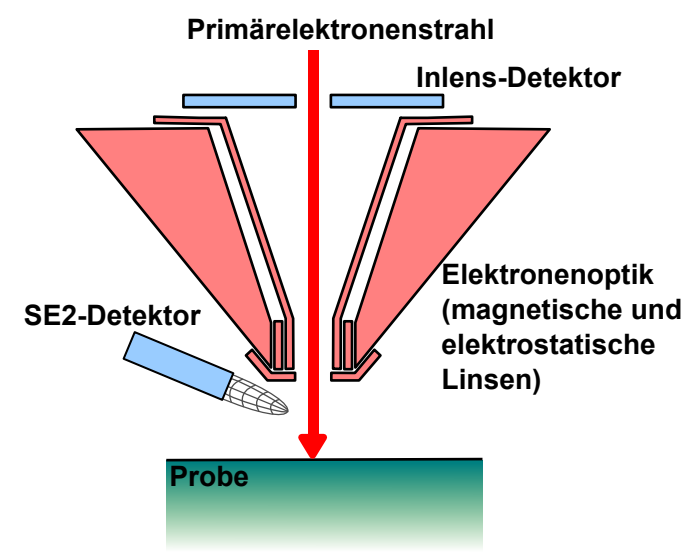

(a) Geometrische Anordung der Detektoren

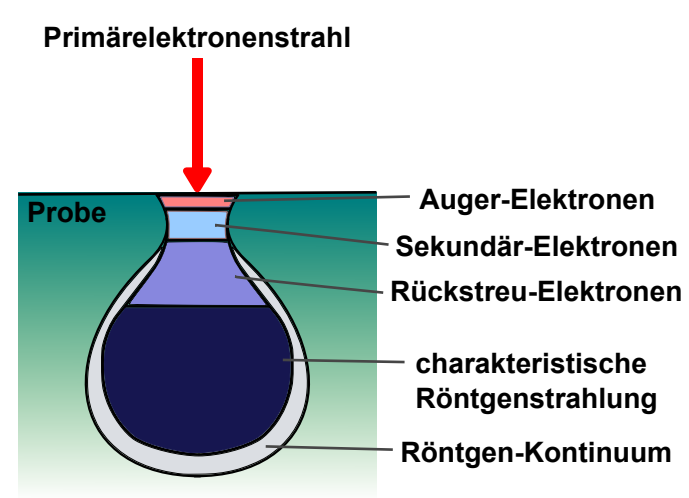

(b) Wechselwirkung des Elektronenstrahls in der Probe

Abb. 4.3: (a) Übersicht über die Lage der Detektoren im Rasterelektronenmikroskop und (b) Schema des Wechselwirkungsvolumens vom Primärelektronenstrahl in dem Probenmaterial nach Ref. [80].

In einem Rasterelektronenmikroskop wird die zu untersuchende Probe mit einem Elektronenstrahl abgerastert. Die bei der Wechselwirkung des Elektronenstrahles mit der Probe entstehenden Produkte wie Sekundärelektronen, Rückstreuelektronen, Röntgenstrahlung

\footnotetext{
${ }^{5}$ Der Begriff Korngröße wird vereinfachend für die Größe des Röntgenstrahlung kohärent streuenden Bereiches (parallel zur Oberflächennormalen der Schicht) verwendet. Eine Verkleinerung des kohärent streuenden Bereiches ergibt sich auch durch ausgedehnte Gitterfehler wie Groß- und Kleinwinkelkorngrenzen, Zwillingsgrenzen und Stapelfehler.
} 
und Kathodolumineszenz werden zur Bildgebung verwendet. Die in dieser Arbeit gezeigten Messungen wurden in einem Leo Supra 35 der Firma Zeiss durchgeführt. Unter Zuhilfenahme des zu dieser Anlage zugehörigen Benutzerhandbuches wurde der nachfolgende Abschnitt erstellt. [80]

Zur Erzeugung des Elektronenstrahls wird eine thermische Feldemissionskathode verwendet, die aus einem Wolfram-Einkristall und einem gesinterten Zirkoniumoxid-Reservoir besteht. Ein Heizstrom schmilzt das Zirkoniumoxid auf. Durch einen Extraktor wird ein elektrisches Feld aufgebaut, das bewirkt, dass am Ende des Wolfram-Einkristalls das Zirkoniumoxid eine sehr feine Spitze ausbildet, aus welcher ein stabiler Elektronenstrahl emittiert wird.

Eine Besonderheit des Leo Supra 35 ist die GEMINI-Optik; es befindet sich innerhalb der Elektronenobjektivlinse ein Strahlbooster. Dieser beschleunigt die Elektronen um zusätzliche $8 \mathrm{kV}$ zu der gewählten Beschleunigungsspannung. Kurz vor dem Verlassen der Objektivlinse werden die Elektronen mit einer elektrostatischen Linse wieder um diesen Betrag abgebremst. Der Strahlbooster hat den Vorteil, dass der Elektronenstrahl innerhalb der Optik weniger anfällig auf elektromagnetische Störfelder ist.

Nach Verlassen des Objektivs trifft der Elektronenstrahl auf die Probe. Hier bilden sich innerhalb eines Wechselwirkungsvolumen (in Abhängigkeit der eingestellten Beschleunigungsspannung und des Probenmaterials) die in Abb. 4.3 gezeigten Sekundärprodukte: Durch inelastische Streuung der Elektronen an den Atomkernen/Elektronenhüllen der Probe entstehen niederenergetische Sekundärelektronen $(E \leq 50 \mathrm{eV})$. Sekundärelektronen Typ I und II entstehen in der Regel in der Nähe der Probenoberfläche. ${ }^{6}$ Durch elastische Streuung kommt es zu Rückstreuelektronen mit Energien von $E \geq 50 \mathrm{eV}$. Des Weiteren wird charakteristische Röntgenstrahlung angeregt, diese wird zur Materialanalyse verwendet. Unterschiedliche Detektoren werden zur Detektion der verschiedenen Sekundärprodukte verwendet. Auch eine Bildgebung durch eine Überlagerung des Signales von unterschiedlichen Detektoren kann sinnvoll sein und ist in der Steuerungssoftware vorgesehen.

Der Inlens-Detektor befindet sich, wie der Name sagt, oberhalb der Objektivlinse. Damit ist die Blickrichtung parallel zum Primärelektronenstrahl und in der Regel senkrecht zur Probenoberfläche. Durch die elektrostatische Linse, die das Gegenfeld zum Strahlbooster erzeugt, werden die Elektronen in das Objektiv und damit zum Inlens-Detektor gelenkt. Dieses führt zu einer erhöhten Elektronenausbeute am Detektor. Der Inlens-Detektor detektiert nahezu ausschließlich Sekundärelektronen Typ I und II, womit die Bildinformationen dieses Detektors relativ oberflächensensitiv sind. Der Inlens-Detektor hat auch das größte Auflösungsvermögen der zur Verfügung stehenden Detektoren und wird daher in dieser Arbeit hauptsächlich zur Untersuchung der Probenoberfläche bei hohen Vergrößerungen verwendet.

Im Gegensatz zum Inlens-Detektor befindet sich der SE2-Detektor außerhalb des Objektivs; damit steht er nicht parallel zum Primärelektronenstrahl, wodurch eine erhöhte Tiefeninformation entsteht. Es werden etwa $90 \%$ Sekundärelektronen Typ I/II und $10 \%$ Rückstreuelektronen (bei Standardeinstellungen) zur Bildgebung verwendet. [80] Damit ist ein erhöhter Materialkontrast als im Inlens gegeben. Zur Materialanalyse kann ein

\footnotetext{
${ }^{6}$ Als Sekundärelektronen Typ I werden Elektronen bezeichnet, die in unmittelbarer Nähe zum Auftreffpunkt des Primärelektronenstrahls durch inelastische Streuprozesse entstehen. Sekundärelektronen Typ II entstehen durch Mehrfachstreuung etwas weiter entfernt. Sekundärelektronen Typ III können durch Rückstreuelektronen weit entfernt von dem Spot angeregt werden.
} 
Rückstreuelektronen-Detektor in den Strahlengang eingeschoben werden. Zudem ist eine EDX-Einheit (energy dispersive X-ray spectroscopy) zur Materialanalyse vorhanden. Hierbei wird das Spektrum der Röntgenstrahlung aufgenommen und anhand der charakteristischen Röntgenstrahlung die in der Probe vorhandenen Elemente bestimmt.

Zur Mikrostrukturierung mittels Elektronenstrahl-Lithographie verfügt das vorgestellte Leo Supra 35 über eine Lithographie-Einheit. Die Details der Mikrostrukturierung werden im nächsten Abschnitt beschrieben.

\subsection{Mikrostrukturierung}

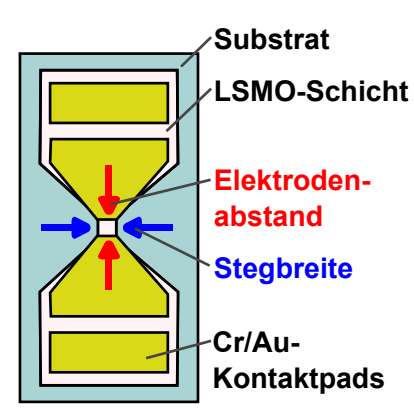

(a) Elektronenstrahl-Lithographie

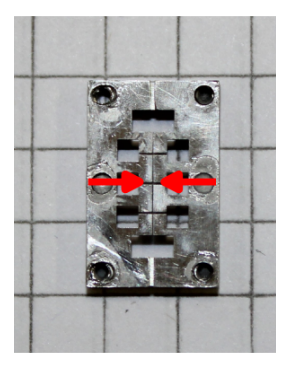

(b) optische Lithographie

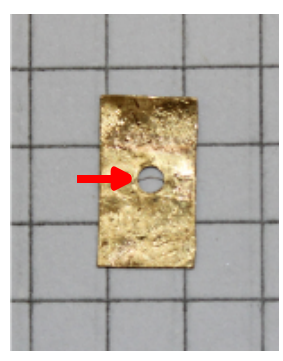

(c) Drahtmaske

Abb. 4.4: Verschiedene Masken zur Mikrostrukturierung.

Eine Mikrostrukturierung der Proben wird mittels Elektronstrahl-Lithographie und optischer Lithographie vorgenommen, wobei die einzelnen Prozessschritte im Folgenden beschrieben werden. Die Details zu den jeweiligen Lacken und Herstellungsbedingungen sind zur Übersicht in Tabelle 4.5 zusammengefasst.

Vor dem ersten Lithographieschritt werden die Proben gereinigt und anschließend für fünf Minuten auf eine Heizplatte bei $180^{\circ} \mathrm{C}$ gelegt, um Wasserfilme auf den Probenoberflächen zu verdampfen. Der Lack (und Haftvermittler) wird mit einem Spincoater (Süss, MicroTec) auf die Proben aufgetragen. Anschließend werden die Proben - je nach Lack - im Konvektionsofen gebacken. Die Belichtung erfolgt bei der Elektronenstrahl-Lithographie in dem vorgestellten Leo Supra 35. Wie zuvor angemerkt, verfügt das REM über eine zusätzliche Lithographie-Einheit (Elphy-Plus der Firma Raith). Für die optische Belichtung werden Wellenlängen im Bereich von $\lambda \approx 360 \ldots 450 \mathrm{~nm}$ verwendet, die verwendete Maske ist in Abb. 4.4(b) gezeigt und wurde im Rahmen der Dissertation von Melanie Schneider angefertigt. Diese Maske ermöglicht eine variable Stegbreite zwischen $20 \ldots 500 \mu \mathrm{m}$. Nach der Belichtung (bei der optischen Lithographie sind weitere Prozessschritte notwendig, siehe Tabelle 4.5) wird der Lack entwickelt, also die belichteten bzw. unbelichteten Bereiche herausgelöst.

Die Lackstrukturen werden mittels Trockenätzen in die dünnen Schichten übertragen. Dazu wird eine Kaufmannquelle (in einer Ultrahochvakuum-Anlage) verwendet, als Arbeitsgas dient Argon. Um ein möglichst gleichmäßiges Ätzresultat zu erzielen, wird die Probe während des Ätzprozesses auf der Transferstange gedreht. Der Ätzvorgang wird der Schichtdicke angepasst und die Schicht - an den Stellen ohne Lack - komplett weggeätzt. Anschließend wird der Lack mittels Aceton im Ultraschallbad abgelöst. 


\begin{tabular}{|c|c|c|c|}
\hline $\begin{array}{l}\text { Prozess- } \\
\text { schritt }\end{array}$ & $\begin{array}{c}\text { Optische } \\
\text { Lithographie }\end{array}$ & $\begin{array}{l}\text { Elektronenstrahl- } \\
\text { Lithographie } \\
\text { (Strukturen) }\end{array}$ & $\begin{array}{l}\text { Elektronenstrahl- } \\
\text { Lithographie } \\
\text { (Kontaktpads) }\end{array}$ \\
\hline $\begin{array}{l}\text { Haftvermittler } \\
\text { Spincoater } \\
\text { Konvektions- } \\
\text { ofen }\end{array}$ & $\begin{array}{c}\text { AR } 300-80 \\
60 \mathrm{~s} \text { bei } 4000 \mathrm{rpm} \\
25 \mathrm{~min} \text { bei } 180^{\circ} \mathrm{C}\end{array}$ & - & - \\
\hline $\begin{array}{l}\text { Lack } \\
\text { Spincoater } \\
\text { Lackdicke }\end{array}$ & $\begin{array}{c}\text { AR-U } 4040 \\
60 \mathrm{~s} \text { bei } 2500 \mathrm{rpm} \\
\approx 2 \mu \mathrm{m}\end{array}$ & $\begin{array}{c}\text { AR-P } 671.09 \\
60 \mathrm{~s} \text { bei } 2500 \mathrm{rpm} \\
\approx 2 \mu \mathrm{m}\end{array}$ & $\begin{array}{l}\text { AR-P } 671.02 \\
60 \mathrm{~s} \text { bei } 2500 \mathrm{rpm} \\
\approx 100 \mathrm{~nm}\end{array}$ \\
\hline $\begin{array}{l}\text { Struktur- } \\
\text { belichtung }\end{array}$ & $7 \mathrm{~min}$ & $\begin{array}{c}235 \mu \mathrm{C} / \mathrm{cm}^{2} \\
\mathrm{U}=20 \mathrm{kV} \\
\text { Blende }=30 \mu \mathrm{m}\end{array}$ & $\begin{array}{c}250 \mu \mathrm{C} / \mathrm{cm}^{2} \\
\mathrm{U}=20 \mathrm{kV} \\
\text { Blende }=30 \mu \mathrm{m}\end{array}$ \\
\hline $\begin{array}{l}\text { Konvektions- } \\
\text { ofen } \\
\text { Flut- } \\
\text { belichtung }\end{array}$ & $\begin{array}{c}30 \mathrm{~min} \text { bei } 85^{\circ} \mathrm{C} \\
\text { (Umkehrtemperung) } \\
18 \mathrm{~min}\end{array}$ & - & - \\
\hline $\begin{array}{l}\text { Entwicklung } \\
\text { Entwicklungs- } \\
\text { stop }\end{array}$ & $\begin{array}{c}\text { AR 300-35: } \mathrm{H}_{2} \mathrm{O}(4: 1) \\
\approx 150 \mathrm{~s} \\
\text { destilliertes Wasser }\end{array}$ & $\begin{array}{c}\text { AR 600-56 } \\
1 \ldots 3 \mathrm{~min} \\
\text { Isopropanol }\end{array}$ & $\begin{array}{c}\text { AR } 600-56 \\
1 \ldots 3 \mathrm{~min} \\
\text { Isopropanol }\end{array}$ \\
\hline
\end{tabular}

Tabelle 4.5: Übersicht der verschiedenen Lithographieprozesse. 
Zur Kontaktierung der Proben wurden Chrom/Gold-Kontakte in einem zweiten Lithographieprozess auf der Probe positioniert. Hierzu werden (mit einem anderen Lack) alle Lithographie-Prozessschritte wiederholt. Auf die Lackmaske werden dann Chrom- und Goldschichten in einer Vakuumkammer der Firma Edwards mittels zweier thermischer Verdampfer gedampft. Die $5 \mathrm{~nm}$ dicke Chromschicht dient zur Haftvermittlung zwischen der Manganat- und Goldschicht. Auf die Chromschicht folgt eine $50 \ldots 100 \mathrm{~nm}$ dicke Goldschicht. Des Weiteren werden Chrom/Gold-Kontakte mit der in Abb. 4.4(c) gezeigten Drahtmaske auf die Proben gebracht.

\subsection{Elektronische Transportmessungen}

Temperaturabhängige, elektronische Transportmessungen werden sowohl im einem PPMS 9 (physical property measurement system) der Firma Quantum Design, als auch in einem MagLab-System ${ }^{2000}$ von Oxford Instruments durchgeführt. In beiden Kryostaten kann in einem Temperaturbereich von $1,5 \ldots 400 \mathrm{~K}$ gemessen werden. ${ }^{7}$ Das externe Magnetfeld kann zwischen $0 \ldots 9 \mathrm{~T}$ variiert werden.

Um Strom-Spannungs-Kennlinien mit Spannungen von bis zu $\pm 20 \mathrm{~V}$ aufzunehmen, werden die Proben in dem MagLab-Kryostaten eingebaut. Die Kennlinien werden mit Hilfe eines Keithley 2400 und eines Keithley 2001 aufgenommen, die teilweise extern oder mit einer in dem MagLab ${ }^{2000}$-System vorhanden Software (Objectbench) angesteuert werden.
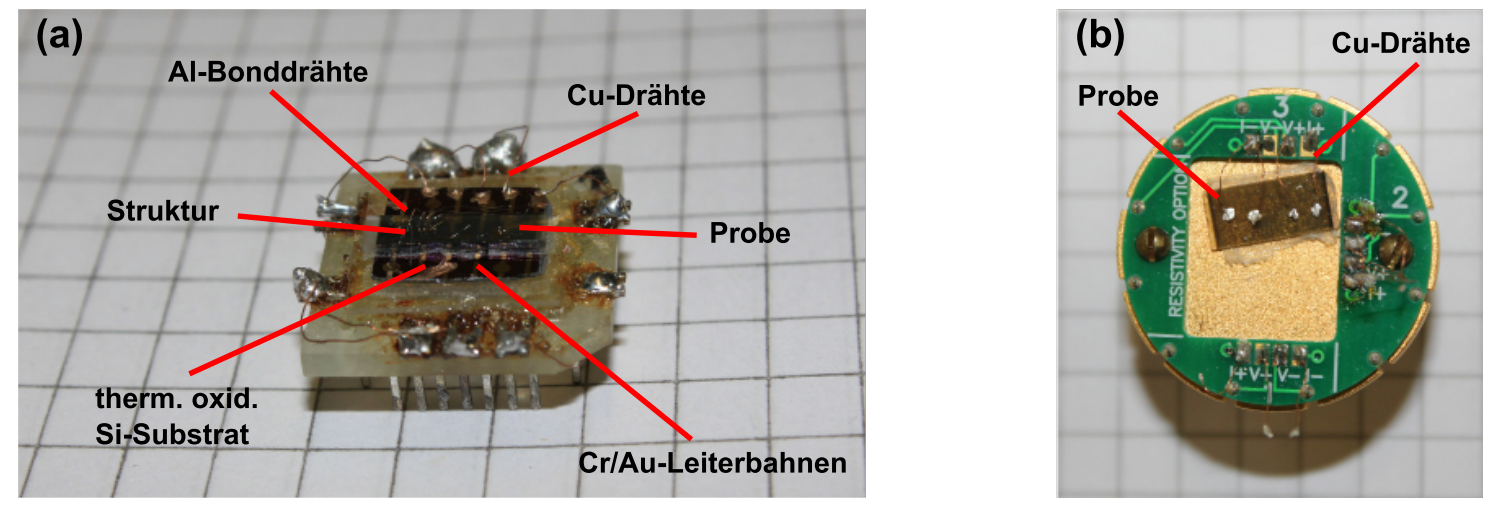

Abb. 4.6: Kontaktierungen von einer strukturierten Schicht im MagLab-Probenhalter (a) und unstrukturierten Probe in einem PPMS-Probenhalter (b).

Für die elektronischen Transportmessung werden die mittels Elektronenstrahl-Lithographie hergestellten Strukturen mit einem Bonder (Model 4532 der Firma Kulicke \& Soffa Ltd.) mit Aluminiumdraht kontaktiert. Um die strukturierten Schichten in verschiedenen Kryostaten messen zu können, werden die Proben auf einem thermisch oxidierten SiliziumSubstrat befestigt. Auf dem Silizium-Substrat sind makroskopische $\mathrm{Cr} / \mathrm{Au}$-Leiterbahnen mit einer Maske gedampft worden. Die Bonds werden zwischen den Strukturen und den Leiterbahnen auf dem Silizium-Substrat angebracht, siehe Abb. 4.6. Die Leiterbahnen auf dem Silizium-Substrat werden mit Kupferdrähten auf dem jeweiligen Probenhalter kontaktiert.

Bei unstrukturierten Proben und größeren Strukturen wie durch optische Lithographie hergestellte Strukturen oder auch die durch die Drahtmaske gedampften Kontakte werden die Kupferdrähte direkt auf die Kontaktpads bzw. die Schicht mit Leitsilber geklebt.

\footnotetext{
${ }^{7}$ Mit einem ${ }^{3}$ He-Spezialeinsatz werden im PPMS sogar Temperaturen von $400 \mathrm{mK}$ erreicht.
} 
Der Widerstand wird hierbei - wenn nicht anders angegeben - mit Vierpunktmessungen bestimmt, wie in Abb. 4.6 zu sehen ist.

\subsection{Magnetische Charakterisierung}

Zur magnetischen Charakterisierung der dünnen Schichten wird ein MPMS XL (magnetic property measurement system) der Firma Quantum Design verwendet. Diese Anlage ermöglicht Messungen in einem Temperaturbereich von 2 . . $400 \mathrm{~K}$ und das Anlegen von magnetischen Felder von bis zu 5 T. Die relative Empfindlichkeit beträgt dabei $1 \cdot 10^{-8} \mathrm{emu}$. [81]

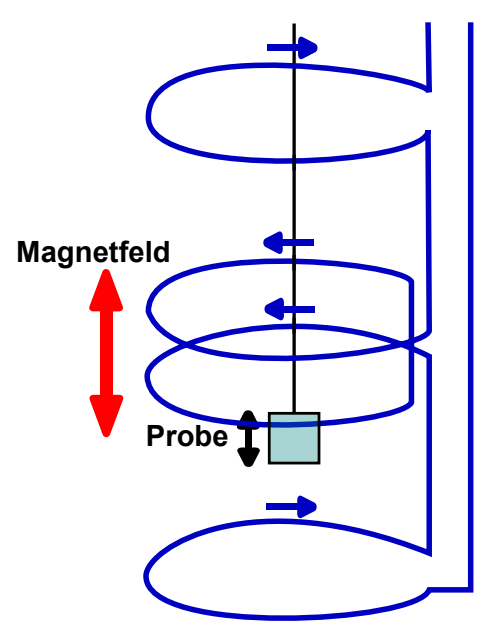

Abb. 4.7: Gradiometer zweiter Ordnung nach Ref. [82].

Zur Messung des magnetischen Moments einer Probe wird diese durch ein Gradiometer zweiter Ordnung bewegt. Das Gradiometer besteht aus einer supraleitenden Spule mit vier Windungen, dabei sind die äußeren Windungen entgegengesetzt zu den inneren beiden Windungen gewickelt, siehe Abb. 4.7. Diese Geometrie sorgt dafür, dass die Detektion relativ unempfindlich auf eine Drift oder ein Rauschen des eingestellten Magnetfeldes ist. [82, 83] Der in dem Gradiometer induzierte Strom wird induktiv in den SQUID-Sensor (superconducting quantum interference device) eingekoppelt. Das SQUID ist durch eine supraleitende Abschirmung von äußeren Magnetfeldern abgeschirmt.

In der verwendeten Anlage handelt es sich um einen RFSQUID (radio frequency SQUID) bestehend aus einem supraleitenden Ring, welcher durch einen Josephson-Kontakt unterbrochen ist. Josephson-Kontakte sind Tunnelkontakte zwischen Supraleitern, die dünn genug sind, um eine schwache Kopplung der Supraleiter zu ermöglichen. Die Quanteninterferenz der makroskopischen Wellenfunktionen der Supraleiter auf beiden Seiten der Barriere und die Flussquantisierung im supraleitenden Ring ermöglichen die sehr empfindliche Messung des magnetischen Moments der zu untersuchenden Probe. $\mathrm{Zu}$ den Grundlagen der Supraleitung wie dem Josephson-Effekt und der Flussquantisierung sei hier lediglich auf weiterführende Literatur verwiesen, siehe beispielsweise Ref. [84, 85, 86].

\subsection{Rastersondenmikroskopie}

Die Rastersondenmikroskopie erlaubt die Charakterisierung von Oberflächen bis hin zu atomarer Auflösung. Eine Sonde wird über die Oberfläche einer Probe gerastert und zeichnet dabei an jedem Punkt des Rasters die lokale Wechselwirkung der Sonde mit der Probe auf; diese wird zur Bildgebung verwendet. Beruhend auf unterschiedlichen Sonden und damit unterschiedlichen Wechselwirkungen zwischen Sonde und Probe werden verschiedene physikalische Eigenschaften abgetastet. Prominente Vertreter der Rastersondenmikroskopie sind Rastertunnel-, Rasterkraft- und Magnetkraftmikroskope. ${ }^{8}$ Entscheidend für eine gute Auflösung bei der Rastersondenmikroskopie ist eine gute Vibrationsdämpfung des Mikroskops.

\footnotetext{
${ }^{8}$ Auch das in Abschnitt 4.3 vorgestellte Rasterelektronenmikroskop kann zu der Rastersondenmikroskopie gezählt werden.
} 
In der vorliegenden Arbeit kommen ein Rastertunnel- und ein Rasterkraftmikroskop zum Einsatz. Bei einem Rastertunnelmikroskop (STM: scanning tunneling microscope) wird als Sonde eine metallische Spitze verwendet. Zwischen Probe und Spitze wird eine Spannung angelegt und die Spitze wird an die Probe angenähert, bis ein zuvor definierter Solltunnelstrom fließt. Im constant height mode wird die Sonde in einer festen Höhe über der Probe gerastert und der Tunnelstrom als lokales Signal aufgenommen. Dieser kann dann in Form einer Tunnelstrom-Karte aufgezeichnet werden. Ein zweiter Messmodus besteht in der Regelung der Höhe der Sonde, hierbei wird der Tunnelstrom - meist über eine piezomechanische Regelung - konstant gehalten (constant current mode). Die eingestellte Höhe der Spitze wird als Funktion des Ortes in Form einer Topographiekarte aufgetragen. Durch die exponentielle Abhängigkeit des Tunnelstroms von dem Abstand können sehr kleine Abstandsänderungen detektiert werden, wodurch das hohe Auflösungsvermögen in einem Rastertunnelmikroskop bedingt ist. In dieser Arbeit ist das verwendete Rastertunnelmikroskop ein Nanoscope IV der Firma Veeco Instruments incorporated. Die Spitze ist ein (von Hand gerissener) Pt/Ir-Draht. Zur Vibrationsdämpfung wird das Mikroskop auf eine an Expanderseilen aufgehängte Granit-Platte gestellt.

Zusätzlich werden im Rahmen dieser Arbeit Rasterkraftmikroskopie-Messungen (AFM: atomic force microscope) durchgeführt. Bei der Rasterkraftmikroskopie wird als Sonde ein Cantilever (deutsch: Biegebalken) verwendet, dessen Spitze im Kontakt-Modus im mechanischen Kontakt mit der Probenoberfläche ist. Entsprechend des STM constant current mode kann mit einer konstanten Kraft bzw. Verbiegung des Cantilevers über die Probenoberfläche gerastert werden. Die Auslenkung bzw. die Verbiegung des Cantilevers wird mit Hilfe eines am Cantilever reflektierten Laserstrahles über einen optischen Positionssensor aufgenommen. Über eine Piezomechanik wird die Verbiegung konstant gehalten und die eingeregelte Höhe zur Bildgebung verwendet.

Die AFM-Messmethode kann erweitert werden, indem ein Cantilever mit einer leitfähigen Beschichtung der Spitze verwendet wird. Wird während eines Topographiescans eine konstante Spannung zwischen Cantilever und Probe angelegt, so kann zusätzlich der lokal fließende Strom aufgenommen werden. So ergeben sich gleichzeitig eine AFM-Topographie und eine Stromkarte vom selben Probenbereich. Wird die elektrische Spannung zwischen Cantilever und Probe variiert, kann der Einfluss unterschiedlicher elektrischer Felder auf der lokalen Skala untersucht werden. Ein Nachteil bei der Verwendung eines beschichteten Cantilevers ist, dass die Spitze des Cantilevers größer als die eines unbeschichteten Cantilevers ist. Dieses bedingt eine schlechtere Auflösung. ${ }^{9}$

Für die AFM-Messungen mit einem leitfähigen Cantilever (C-AFM: conductive-AFM) wird ein VT-AFM/STM der Omicron Nanotechnologie GmbH verwendet. Dieses ermöglicht AFM- und STM-Messungen in einem Temperaturbereich von $25 \ldots 750 \mathrm{~K}$. Betrieben wird das Mikroskop in einer Ultrahochvakuum-Anlage (Enddruck $10^{-10} \mathrm{mbar}$ ). Zur Vibrationsdämpfung ist das Mikroskop an vier Federn aufgehängt (Resonanzfrequenz $2 \mathrm{~Hz}$ ). Um Schwingungen zu dämpfen, sind an der Bodenplatte des Mikroskops Kupferplättchen befestigt, die zwischen festen, äußeren Permanentmagneten angeordnet sind. Bewegt sich bzw. schwingt das Mikroskop, so werden Wirbelströme in den Kupferplättchen induziert, wodurch Schwingungen gedämpft werden (Stichwort: Wirbelstrombremse).

${ }^{9}$ Die hier verwendeten Cantilever (DPE17 von Mikromasch) bestehen aus mit Platin beschichtetem Silizium; der sphärische Spitzenradius beträgt laut Hersteller $30 \ldots 40 \mathrm{~nm}$. 


\begin{tabular}{lccc}
\hline Laser & Wellenlänge & Energie & Repetitionsrate \\
\hline \hline Diodenlaser & $638 \mathrm{~nm}$ & $1,96 \mathrm{eV}$ & - \\
Femtosekundenlaser & $795 \mathrm{~nm}$ & $1,56 \mathrm{eV}$ & $75 \pm 3 \mathrm{MHz}$ \\
\hline
\end{tabular}

Tabelle 4.8: Übersicht der verwendeten Laser.

\subsection{Photoinduzierte, elektrische Transportmessungen}

Photoinduzierte, elektrische Transportmessungen werden in einem bestehenden Aufbau von Marvin Walter durchgeführt, in dem ein Laserstrahl gezielt auf eine Struktur fokussiert werden kann. Um den Einfluss des Laserlichts auf die erste und dritte harmonische Spannung zu untersuchen, werden die Proben nach der in Abb. 4.4(a) gezeigten Vorlage mikrostrukturiert. Der Laserstrahl wird auf den Steg zwischen den beiden Kontaktpads fokussiert. Um das Messsignal möglichst rauscharm zu halten, werden innerhalb des Aufbaus nur Koaxialkabel verwendet. Die Kontaktierung der Mikrostruktur zum Probenhalter ist allerdings ausschließlich ungeschirmt mit Aluminium-Bonddrähten möglich.

Zur optischen Anregung der Manganatschicht wird der im Aufbau vorhandene Diodenlaser (Toptica Photonics, iBeam Smart) im CW-Betrieb (continuous wave) und zusätzlich ein gepulster Femtosekundenlaser (Femto Laser, Fusion 20150 ) verwendet. Die Details der Laser sind in Tabelle 4.8 zusammengefasst. Der Femtosekundenlaser wird über eine Glasfaser (photonischer Kristall, LMA-PM-15) in den Strahlengang eingekoppelt.

Um die Temperatur der Proben variieren zu können, wird eine Kühlung bzw. Heizung mittels Peltierelementen in den bestehenden Aufbau integriert. Diese ist in Abb. 4.9 gezeigt. Die Probe wird auf das Peltierelement I mit Leitsilber geklebt. Allerdings ist die

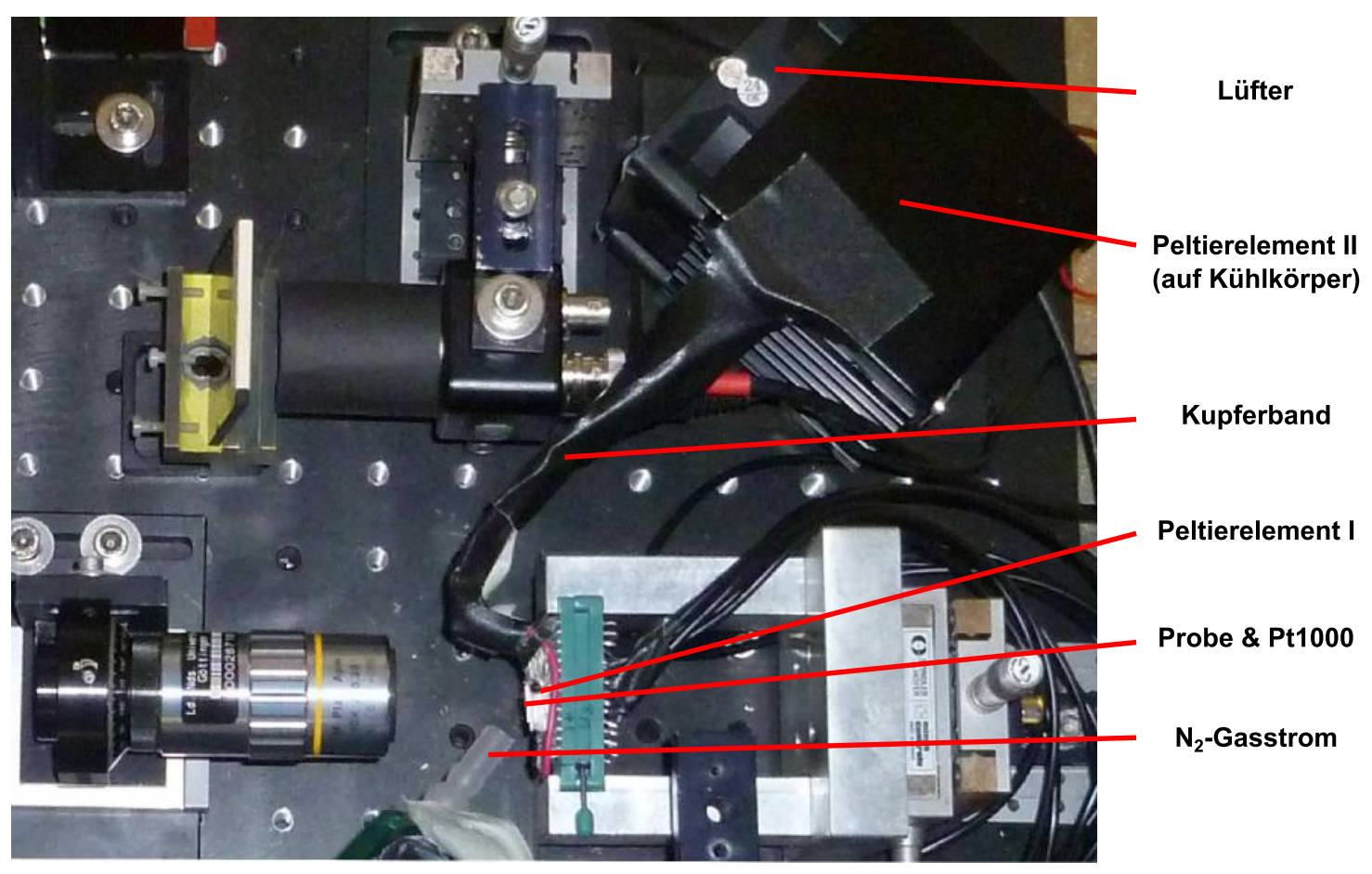

Abb. 4.9: Fotographie der Kühlungs- und Heizungsstufe. 


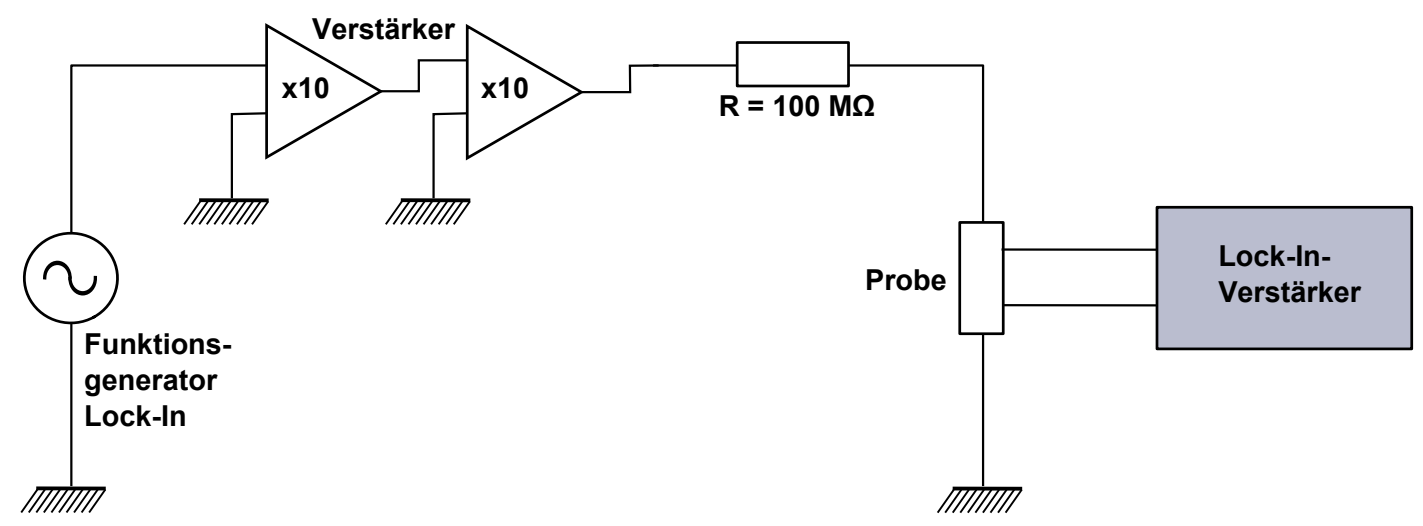

Abb. 4.10: Schaltskizze der elektrischen Messung.

Kühlleistung dieses Peltierelements sehr begrenzt, da aufgrund des geringen Platzes nur ein sehr kleines Peltierelement verwendet werden kann. Des Weiteren ist es schwierig, die Abwärme von der Rückseite des Elementes abzuleiten. Daher ist das Peltierelement I auf einer Kupferplatte befestigt, die mit einem Kupferband im thermischen Kontakt mit einem zweiten, größeren Peltierelement (Peltierelement II) ist. Das Peltierelement II ist wiederum auf einem großen Kühlkörper befestigt, welcher zusätzlich mit einem Lüfter gekühlt wird. Neben der zu untersuchenden Probe befindet sich auf dem Peltierelement I zur Temperaturüberwachung ein Pt1000. Die durch das Pt1000 bestimmte Temperatur entspricht allerdings nur in grober Näherung der Temperatur der Schicht, da der thermische Kontakt von der Schicht bzw. dem Pt1000 zum Peltierelement unterschiedlich sein kann.

Um Kondensation auf der Probenoberfläche zu vermeiden, wird während des Kühlprozesses $\mathrm{N}_{2}$-Gas über die Probe geblasen. Dafür wird eine Kanne mit flüssigem Stickstoff verwendet. In diesem Aufbau werden Temperaturen zwischen $270 \ldots 320 \mathrm{~K}$ erreicht, je nachdem wie gut die thermische Ankopplung zwischen den verschiedenen Elementen ist.

Die Messung des elektrischen Signals $\left(U_{\omega}, U_{3 \omega}\right)$ ist in Abb. 4.10 schematisch gezeigt. Zur Messung des dritten harmonischen Spannungssignals wird eine konstante WechselstromQuelle benötigt. Hierfür wird der interne Sinus-Funktionsgenerator (konstante Wechselspannung) eines Lock-In-Verstärkers (Stanford SR830) als Ausgangssignal verwendet; die maximale Spannungsamplitude ist $5 \mathrm{~V}$. Dieses Ausgangssignal wird in zwei Stufen jeweils um den Faktor zehn verstärkt und in Reihe mit einem großen Widerstand $(R=100 \mathrm{M} \Omega$ ) geschaltet. Der Probenwiderstand liegt in der Größenordnung von $100 \mathrm{k} \Omega$ und ist somit um einen Faktor $10^{3}$ kleiner. Die beobachteten Änderungen des Probenwiderstandes verändern damit den Stromfluss nur in einem vernachlässigbar kleinen Bereich.

Das Messsignal wird mit Hilfe von zwei Lock-In-Verstärkern gemessen: Einer misst dabei auf der ersten harmonischen, der zweite auf der dritten harmonischen Spannung. Es ist zu beachten, dass die Lock-In-Verstärker Effektivwerte ausgeben. Mittels eines vorhandenen Labview-Messprogramms (von Marvin Walter) werden $U_{\omega}, U_{3 \omega}, \theta_{\omega}, \theta_{3 \omega}$ als Funktion der Zeit aufgenommen. 


\section{Charakterisierung von $\mathrm{La}_{0,7} \mathrm{Sr}_{0,3} \mathrm{MnO}_{3} / \mathrm{Al}_{2} \mathrm{O}_{3}$}

In diesem Kapitel werden die mittels der Metallorganischen Aerosol Deposition hergestellten $\mathrm{La}_{0,7} \mathrm{Sr}_{0,3} \mathrm{MnO}_{3}$-Schichten (LSMO) auf $\mathrm{Al}_{2} \mathrm{O}_{3}$-Substraten charakterisiert. Es werden die Ergebnisse von Röntgendiffraktometrie-, Rastertunnelmikrosokopie-, Magnetisierungsund elektrischen Transportmessungen an $40 \ldots 60 \mathrm{~nm}$ dicken Schichten vorgestellt. Die Ergebnisse der Röntgendiffraktometrie zeigen den polykristallinen Charakter der Schichten. Um den Einfluss der Mikrostruktur auf die Schichteigenschaften zu analysieren, werden zusätzlich die Ergebnisse von LSMO/MgO-Schichten vorgestellt, die als epitaktische Vergleichsproben dienen (das Schichtwachstum und Transportverhalten von LSMO/MgO wurde in früheren Studien analysiert) [71, 87]. Des Weiteren wird der Einfluss einer veränderten Schichtdicke $(10 \ldots 300 \mathrm{~nm})$ auf die strukturellen, elektronischen und magnetischen Probeneigenschaften der polykristallinen Schichten untersucht.

Abschnitt 5.5 diskutiert die gewonnenen Ergebnisse, wobei die elektronischen und magnetischen Schichteigenschaften im Rahmen von spinpolarisiertem Tunneln zwischen den Körnern und dem core shell-Modell erklärt werden. Im Rahmen des core shell-Modells werden dem Korninneren und dem Kornäußeren unterschiedliche physikalische Eigenschaften zugeschrieben; hiermit können die Messergebnisse an den LSMO $/ \mathrm{Al}_{2} \mathrm{O}_{3}$-Proben verstanden werden.

Vorherige Arbeiten an mittels MAD hergestellten LSMO/ $\mathrm{Al}_{2} \mathrm{O}_{3}$-Schichten sind insbesondere von Esseling und Köster durchgeführt worden. Auf dieser Grundlage basieren die im Folgenden beschriebenen Ergebnisse. [88, 89, 90]

\subsection{Struktur}

Die Struktur der LSMO-Schichten wird mittels Röntgendiffraktometrie untersucht. Die Ergebnisse von Weitwinkelmessungen der Schichten auf MgO- und $\mathrm{Al}_{2} \mathrm{O}_{3}$-Substraten sind in Abb. 5.1 zu finden. Für die LSMO/MgO-Schichten wird ausschließlich die (001)-Orientierung beobachtet, als Gitterkonstante wird $c=3,877 \pm 0,006 \AA$ bestimmt. Für LSMO $/ \mathrm{Al}_{2} \mathrm{O}_{3}$ dagegen finden sich Intensitätsmaxima für die (001)-, (011)- und (111)-Orientierungen (pseudokubische Darstellung): Die Schicht ist polykristallin. Als mittlere Gitterkonstante (bestimmt aus allen Schichten und Orientierungen) wird $c=3,863 \pm 0,007 \AA$ berechnet, 


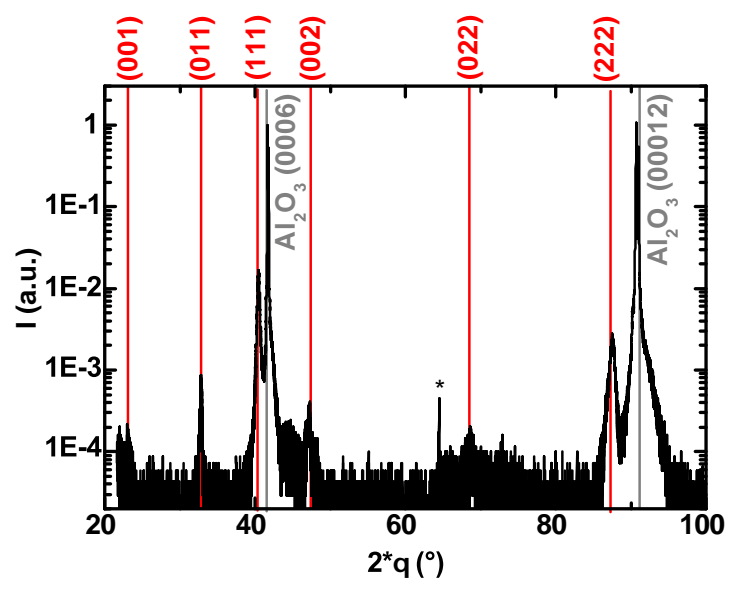

(a) $\mathrm{LSMO} / \mathrm{Al}_{2} \mathrm{O}_{3}$

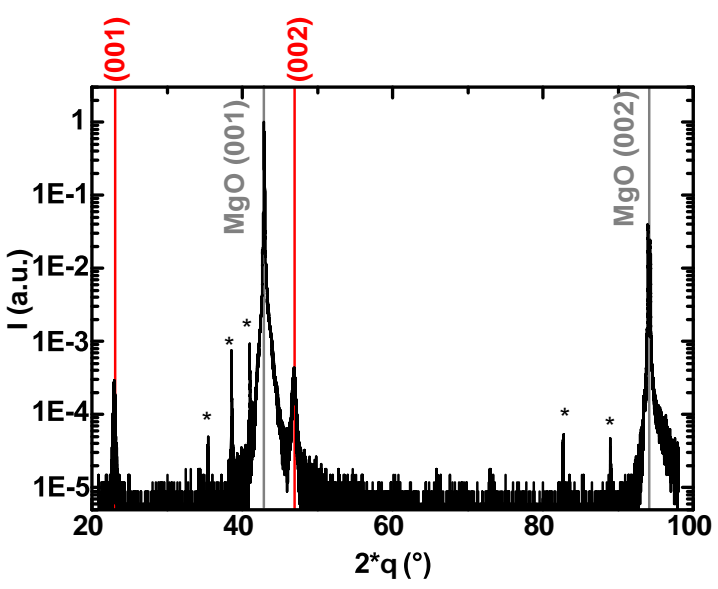

(b) $\mathrm{LSMO} / \mathrm{MgO}$

Abb. 5.1: Röntgendiffraktogramm von $\mathrm{La}_{0,7} \mathrm{Sr}_{0,3} \mathrm{MnO}_{3}$ auf $\mathrm{Al}_{2} \mathrm{O}_{3}$ - und MgO-Substrat: Für $\mathrm{LSMO} / \mathrm{Al}_{2} \mathrm{O}_{3}$ finden sich Intensitätsmaxima für die (001)-, (011)- und (111)-Orientierungen, bei LSMO/MgO dagegen tritt nur die (001)-Orientierung auf. Die mittels eines Sterns gekennzeichneten Maxima können dem Aluminium-Probenhalter und den Substraten zugeordnet werden.

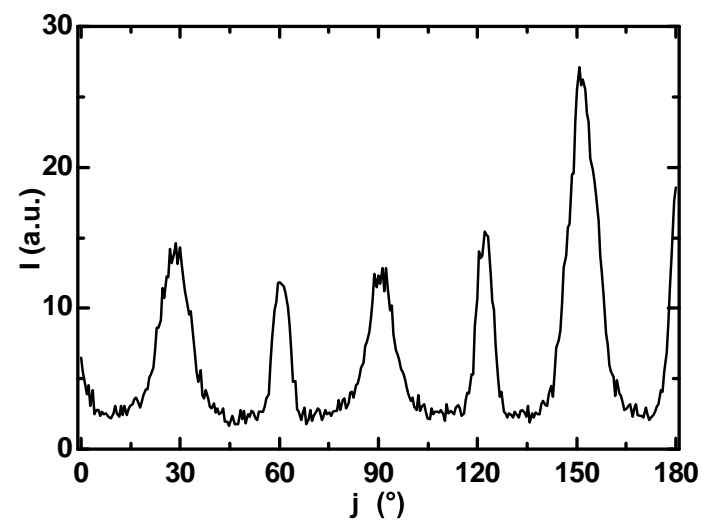

Abb. 5.2: $\varphi$-Scan der (111)-orientierten Körner einer $\mathrm{La}_{0,7} \mathrm{Sr}_{0,3} \mathrm{MnO}_{3} / \mathrm{Al}_{2} \mathrm{O}_{3}$-Schicht (Schichtdicke $d=180 \pm 10 \mathrm{~nm}, 2 \theta_{321}=96,71^{\circ}, \theta_{321,111}^{\prime}=70,56^{\circ}$ ). In einem Winkelbereich zwischen $0 \ldots 180^{\circ}$ treten sechs Intensitätsmaxima auf.

wobei der angegebene Fehler der Standardabweichung entspricht. Im Rahmen der Messgenauigkeit ergeben sich keine unterschiedlichen Gitterkonstanten für die verschiedenen Orientierungen. Auf eine Auswertung der Halbwertsbreite der Intensitätsmaxima wird verzichtet, da die Aufspaltung der $\mathrm{K}_{\alpha 1^{-}}$und $\mathrm{K}_{\alpha 2}$-Linien nicht aufgelöst werden konnte und die instrumentelle Verbreiterung unbekannt ist. Zudem ist der (111)-Schichtpeak (größte Intensität) mit dem Substrat-Peak $\left(\mathrm{Al}_{2} \mathrm{O}_{3}\right.$ (0006)) überlagert, welches eine Auswertung weiter erschwert.

Um die Textur der LSMO/ $/ \mathrm{Al}_{2} \mathrm{O}_{3}$-Schichten zu analysieren, wird ein $\varphi$-Scan der (111)orientierten Körner durchgeführt. Die $\varphi$-Messung einer $180 \pm 10 \mathrm{~nm}$ dicken $\mathrm{LSMO} / \mathrm{Al}_{2} \mathrm{O}_{3}$ - 
Schicht ist in Abb. $5.2 \mathrm{zu}$ sehen. Hierbei finden sich sechs Intensitätsmaxima in einem Winkelbereich zwischen $0 \ldots 180^{\circ}$.

\subsection{Oberflächenmorphologie}

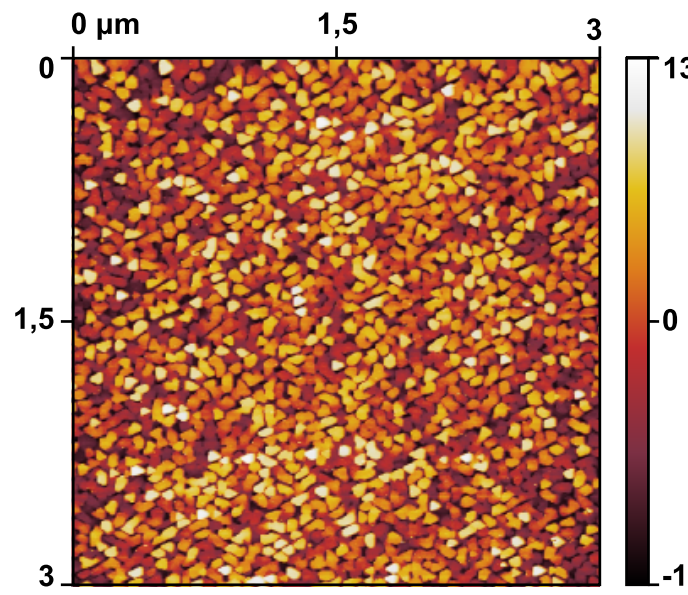

(a) $\mathrm{LSMO} / \mathrm{Al}_{2} \mathrm{O}_{3}$

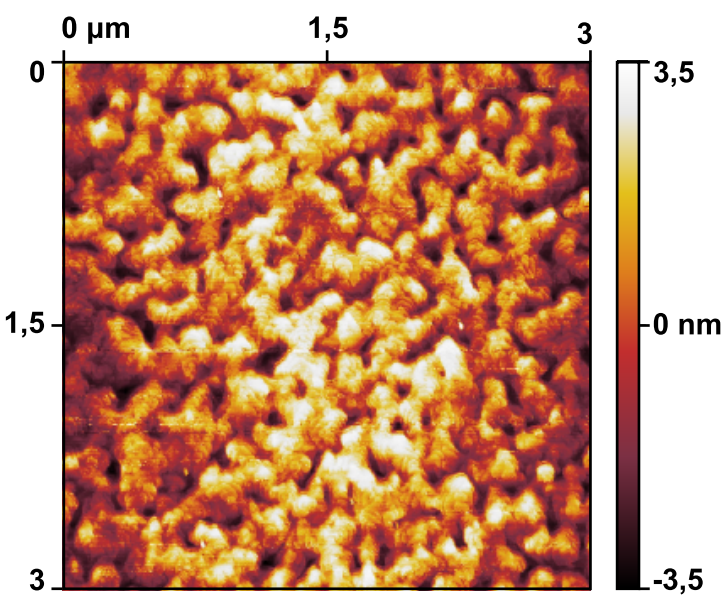

(b) $\mathrm{LSMO} / \mathrm{MgO}$

Abb. 5.3: STM-Aufnahmen: Morphologie von $\mathrm{La}_{0,7} \mathrm{Sr}_{0,3} \mathrm{MnO}_{3}$ auf $\mathrm{Al}_{2} \mathrm{O}_{3^{-}}$und $\mathrm{MgO}-$ Substraten.

In Abb. 5.3 sind STM-Aufnahmen von Manganatschichten auf $\mathrm{Al}_{2} \mathrm{O}_{3}$ - und MgO-Substraten zu sehen. Die Schichtmorphologien auf den verschiedenen Substraten sind sehr unterschiedlich. Auf MgO-Substraten zeigen die Schichten (nach Optimierung der Depositionsparameter) Terrassen zwischen denen die Stufenhöhen nur ganze Vielfache von 0,4 nm, der Höhe einer Einheitszelle, sind. Entsprechend sind diese Schichten viel glatter als die polykristallinen $\mathrm{LSMO} / \mathrm{Al}_{2} \mathrm{O}_{3}$-Schichten. Für auf $\mathrm{MgO}$ gewachsene Schichten beträgt die Schichtrauigkeit RMS $=0,76 \mathrm{~nm}$, während für $\mathrm{LSMO} / \mathrm{Al}_{2} \mathrm{O}_{3} \mathrm{RMS}=2,59 \mathrm{~nm}$ ist. Hierbei wurde der RMS auf einer quadratischen Fläche mit einer Kantenlänge von $3 \mu \mathrm{m}$ bestimmt.

In den STM-Messungen der LSMO/ $\mathrm{Al}_{2} \mathrm{O}_{3}$-Schichten zeigt sich in der Schichtrauigkeit der polykristalline Charakter. Es können insbesondere dreieckige Körner beobachtet werden, die der (111)-Orientierung zugeordnet werden können. Ein Linienprofil über mehrere Körner befindet sich in Abb. 5.4. Zur Bestimmung der durchschnittlichen Korngröße $k$ wird
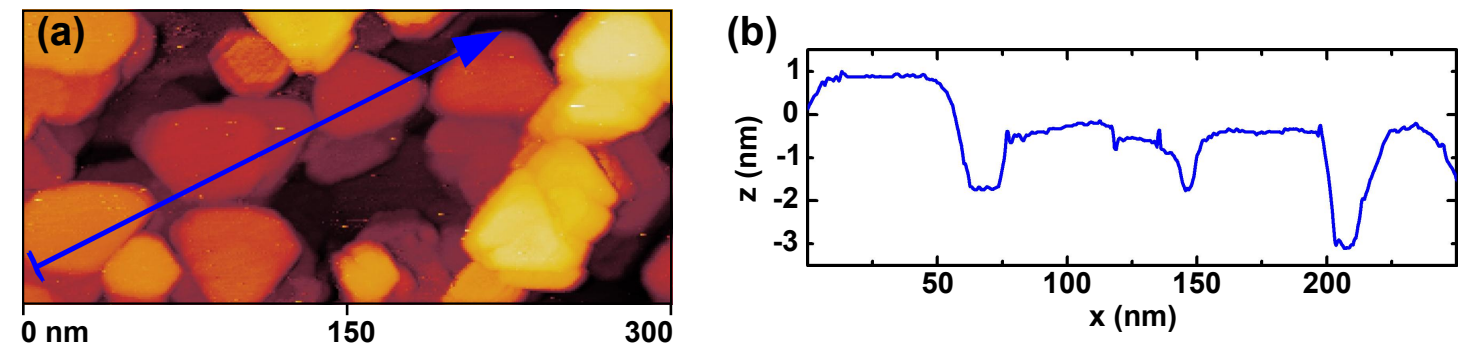

Abb. 5.4: STM-Messung: (a) Topographie einer LSMO/ $\mathrm{Al}_{2} \mathrm{O}_{3}$-Schicht, (b) zugehöriges Höhenprofil. 
die Länge der Körner durch Linienprofile ausgemessen (bei Dreiecken wurde die Höhe bestimmt). Für die in Abb. 5.3(a) gezeigte $53 \mathrm{~nm}$ dicke Schicht wird eine durchschnittliche Korngröße von $k=66 \pm 20 \mathrm{~nm}$ gemessen, wobei der angegebene Fehler der Standardabweichung entspricht. Es ist zu beachten, dass die durchschnittliche Korngröße abhängig von der Schichtdicke ist, wie im Abschnitt 5.4 gezeigt wird.

\subsection{Elektronischer Transport und Magnetisierung}

Die im folgenden Abschnitt gezeigten elektronischen Transport- und Magnetisierungsmessungen stammen von zwei Schichten, die gleichzeitig im selben MAD-Prozess hergestellt wurden. Dazu wurde ein $\mathrm{Al}_{2} \mathrm{O}_{3}$ - und $\mathrm{MgO}$-Substrat nebeneinander auf dem Heizer befestigt. Entsprechend haben beide Proben die gleiche Schichtdicke von $42 \ldots 43 \mathrm{~nm}$ (bestimmt aus Kleinwinkelröntgenreflektometrie). In Abb. 5.5 sind die Temperaturabhängigkeit des Widerstandes und der Magnetisierung dieser Schichten zu sehen.

Das elektronische Transportverhalten von LSMO/ $\mathrm{Al}_{2} \mathrm{O}_{3}$ und LSMO/MgO ist deutlich unterschiedlich: Die Metall-Isolator-Übergangstemperatur liegt für LSMO $/ \mathrm{Al}_{2} \mathrm{O}_{3}$ bei $T_{\mathrm{MI}}=$ $253 \pm 1 \mathrm{~K}$ und für $\mathrm{LSMO} / \mathrm{MgO}$ bei $T_{\mathrm{MI}}=382 \pm 1 \mathrm{~K}$. Hierbei wurde $T_{\mathrm{MI}}$ als Temperatur des globalen Widerstandmaximums definiert. Des Weiteren findet bei der polykristallinen Probe unterhalb von $T_{\mathrm{MI}}$ ein zusätzlicher Vorzeichenwechsel in $d R / d T$ statt. Dieser wird bei der LSMO/MgO-Probe nicht beobachtet. Der Vorzeichenwechsel bzw. das lokale Minimum des Widerstands liegt bei $T_{\min }=29 \pm 1 \mathrm{~K}$. Auch die spezifischen Widerstände weichen deutlich voneinander ab. Während für die auf $\mathrm{MgO}$ deponierte Schicht $\rho(T=50 \mathrm{~K})=8 \cdot 10^{-5} \Omega \mathrm{cm}$ ist, hat die auf $\mathrm{Al}_{2} \mathrm{O}_{3}$ gewachsene Schicht einen spezifischen Widerstand von $\rho(T=50 \mathrm{~K})=3 \cdot 10^{-2} \Omega \mathrm{cm}$. Die Curie-Temperaturen, die über das Minimum der normierten Ableitung $(1 / M) \cdot(\partial M / \partial T)$ bestimmt werden, liegen bei $T_{\mathrm{C}}\left(\mathrm{LSMO} / \mathrm{Al}_{2} \mathrm{O}_{3}\right)=357 \pm 2 \mathrm{~K}$ und $T_{\mathrm{C}}(\mathrm{LSMO} / \mathrm{MgO})=352 \pm 2 \mathrm{~K}$.

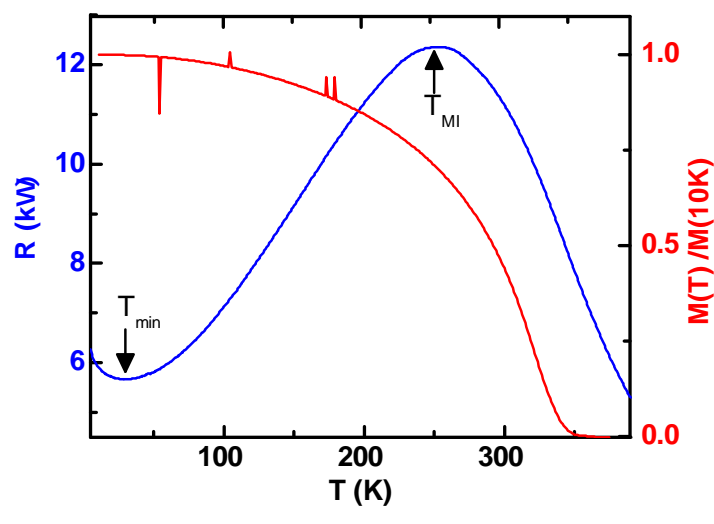

(a) $\mathrm{LSMO} / \mathrm{Al}_{2} \mathrm{O}_{3}$

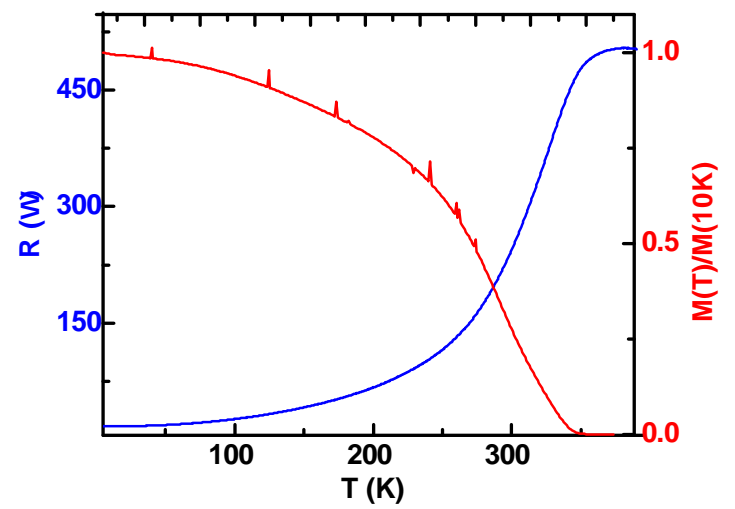

(b) $\mathrm{LSMO} / \mathrm{MgO}$

Abb. 5.5: Widerstand und Magnetisierung von LSMO/ $\mathrm{Al}_{2} \mathrm{O}_{3}$ und LSMO/MgO als Funktion der Temperatur. Die Magnetisierung wurde in einem Feld von 100 Oe (field in plane) gemessen. Für die Metall-Isolator-Übergangstemperatur ergibt sich $T_{\mathrm{MI}}\left(\mathrm{LSMO} / \mathrm{Al}_{2} \mathrm{O}_{3}\right)=253 \pm 1 \mathrm{~K}$ und $T_{\mathrm{MI}}(\mathrm{LSMO} / \mathrm{MgO})=382 \pm 1 \mathrm{~K}$. Die CurieTemperaturen liegen bei $T_{\mathrm{C}}\left(\mathrm{LSMO} / \mathrm{Al}_{2} \mathrm{O}_{3}\right)=357 \pm 2 \mathrm{~K}$ und $T_{\mathrm{C}}(\mathrm{LSMO} / \mathrm{MgO})=$ $352 \pm 2 \mathrm{~K}$. 


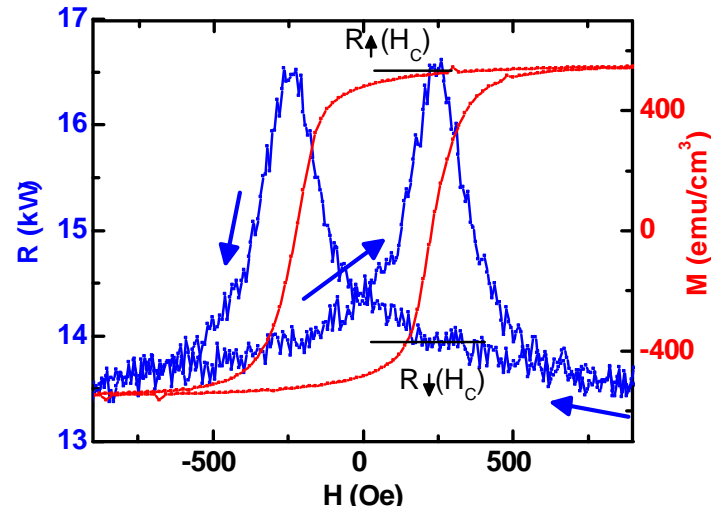

(a) $\mathrm{LSMO} / \mathrm{Al}_{2} \mathrm{O}_{3}$

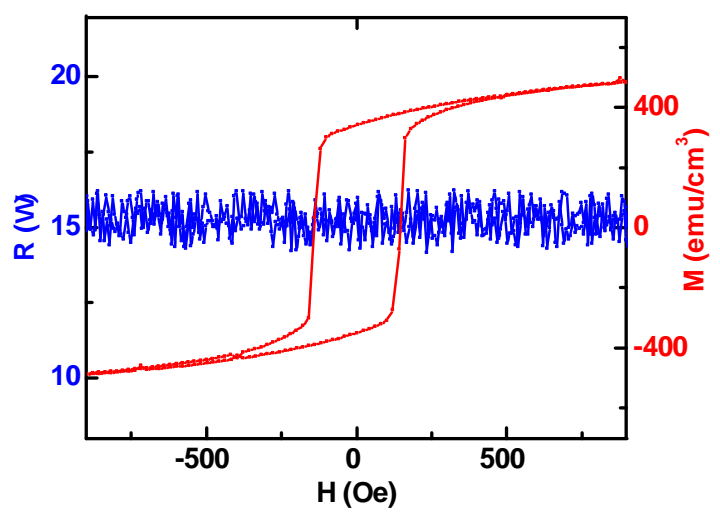

(b) $\mathrm{LSMO} / \mathrm{MgO}$

Abb. 5.6: Magnetowiderstand und magnetische Hysterese von LSMO auf $\mathrm{Al}_{2} \mathrm{O}_{3}$ und $\mathrm{MgO}$ ( $T=10 \mathrm{~K}$, field in plane). Für $\mathrm{LSMO} / \mathrm{Al}_{2} \mathrm{O}_{3}$ wird ein deutlicher Kleinfeldmagnetowiderstand beobachtet $(\mathrm{LFMR}=16,9 \%)$. Die Koerzitivfelder liegen bei $H_{\mathrm{C}}\left(\mathrm{LSMO} / \mathrm{Al}_{2} \mathrm{O}_{3}\right)=230 \pm 10$ Oe und $H_{\mathrm{C}}(\mathrm{LSMO} / \mathrm{MgO})=140 \pm 10$ Oe.

In Abb. 5.6 sind der Magnetowiderstand und die magnetische Hysteresekurve der Manganatschichen auf $\mathrm{Al}_{2} \mathrm{O}_{3}$ und $\mathrm{MgO}$ gezeigt. Messungen des Magnetowiderstands zeigen bei den LSMO $/ \mathrm{Al}_{2} \mathrm{O}_{3}$-Schichten eine deutliche Abhängigkeit von der magnetischen Vorgeschichte: In Abhängigkeit des zuvor angelegten Magnetfeldes treten Maxima im Widerstandsverlauf auf (Kleinfeldmagnetowiderstand, LFMR: low field magnetoresistance). Dieses wird für die LSMO/MgO-Proben nicht beobachtet. Die Widerstandsmaxima stimmen mit den Koerzitivfeldern $H_{\mathrm{C}}$, bestimmt aus den magnetischen Hysteresen, überein. Für LSMO $/ \mathrm{Al}_{2} \mathrm{O}_{3}$ ist $H_{\mathrm{C}}=230 \pm 10 \mathrm{Oe}$, während für LSMO $/ \mathrm{MgO} H_{\mathrm{C}}=140 \pm 10$ Oe ist. Als Maßstab für die Größe des Kleinfeldmagnetowiderstands bzw. für das Verhältnis der verschiedenen Widerstände beim Koerzitivfeld $\left(R \uparrow\left(H_{\mathrm{C}}\right), R \downarrow\left(H_{\mathrm{C}}\right)\right.$, siehe Abb. 5.6(a)) wird der LFMR eingeführt, der über

$$
\mathrm{LFMR}=\frac{R \uparrow\left(H_{\mathrm{C}}\right)-R \downarrow\left(H_{\mathrm{C}}\right)}{R \downarrow\left(H_{\mathrm{C}}\right)} \times 100 \%
$$

definiert ist. Für die in Abb. 5.6 gezeigte, $42 \ldots 43 \mathrm{~nm}$ dünne Schicht ist LFMR =16,9\%. Eine genaue Bestimmung der Sättigungsmagnetisierung ist nicht möglich, da das Probenvolumen nicht genau bestimmt werden konnte: Die Proben wurden zur Befestigung auf dem MAD-Heizer teilweise abgedeckt, daher konnte aufgrund von Abschattungseffekten keine klare Schichtgrenze bestimmt werden.

\subsection{Einfluss der Schichtdicke}

Um den Einfluss der Schichtdicke auf die LSMO/ $\mathrm{Al}_{2} \mathrm{O}_{3}$-Proben zu untersuchen, wurde eine Schichtserie mit Schichtdicken zwischen $10 \ldots 300 \mathrm{~nm}$ hergestellt. Hierbei wurde bei der Präparation der Schichten lediglich die Menge der deponierten Lösung verändert; alle anderen Depositionsparameter wurden konstant gehalten. Es muss allerdings beachtet werden, dass durch die unterschiedliche Menge an Lösung die Depositionszeit und die Depositionstemperatur während der Schichtherstellung verändert werden. 

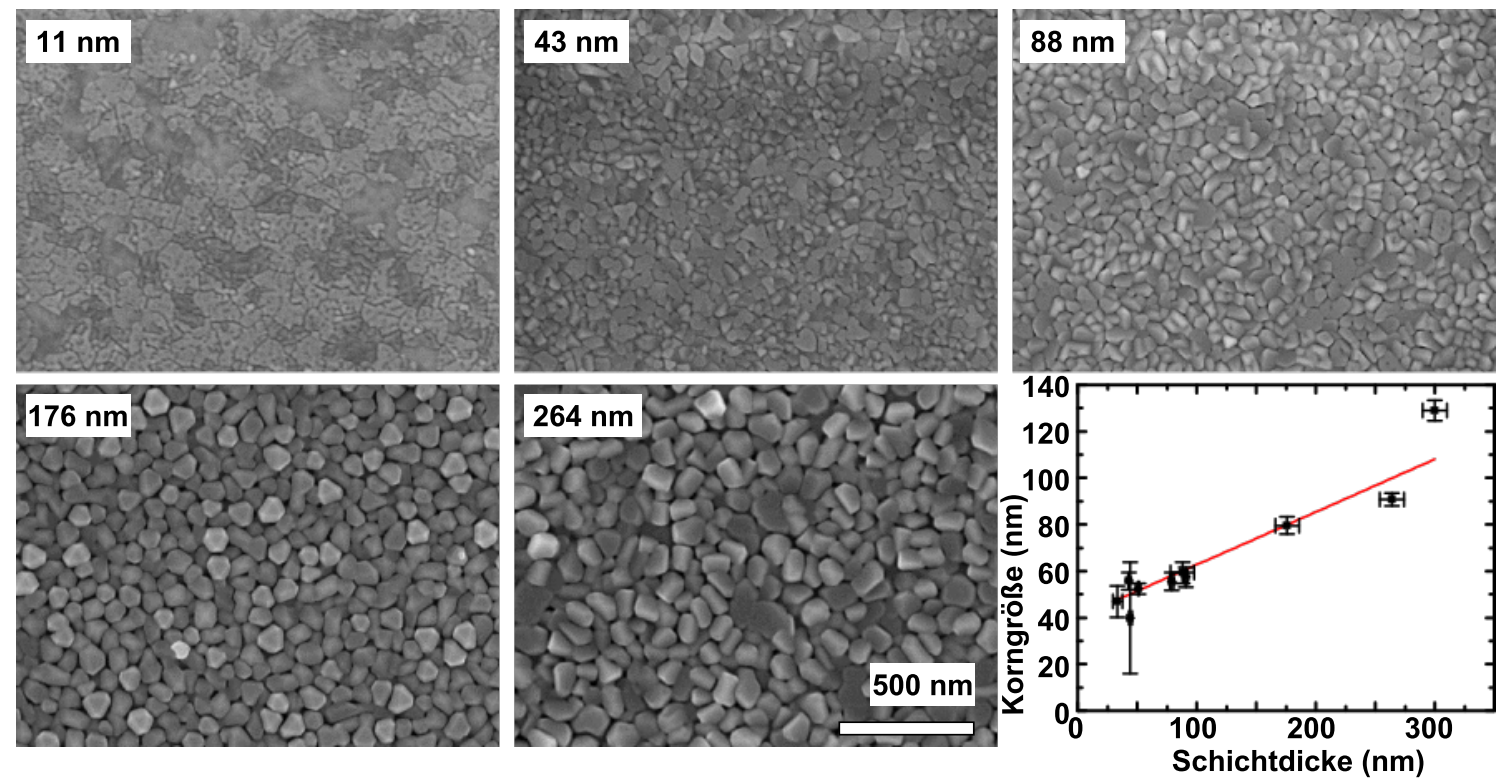

Abb. 5.7: Rasterelektronenmikroskopie-Messungen an LSMO/ $\mathrm{Al}_{2} \mathrm{O}_{3}$-Schichten unterschiedlicher Schichtdicke (Inlens-Detektor, $200 \mathrm{kx}$-Vergrößerung). Der Graph zeigt die durchschnittliche Korngröße $k^{\prime}$ als Funktion der Schichtdicke $d$ mit einem linearen Fit der Form $k^{\prime}(d)=(40 \pm 4 \mathrm{~nm})+(0,23 \pm 0,03) \cdot d$.

Abb. 5.7 zeigt Rasterelektronenmikroskop-Aufnahmen einiger Schichten unterschiedlicher Dicke. Mit steigender Schichtdicke wächst die laterale Ausdehnung der einzelnen Körner. Dieses wird in dem in der Abb. 5.7 gezeigten Graphen verdeutlicht, in dem die mittlere Korngröße (bzw. die durchschnittliche, laterale Ausdehnung) als Funktion der Schichtdicke dargestellt ist. Im Gegensatz zu Abschnitt 5.2 wurde hierbei die Korngröße bestimmt, indem die Zahl der Körner $z$ innerhalb einer bestimmten Fläche $A$ (der REM-Aufnahmen) ausgezählt wurde. Die Korngröße wird daraus über $k^{\prime}=2 \cdot \sqrt{(A /(z \pi)}$ berechnet. ${ }^{1}$ Der in den Abb. 5.7 eingezeichnete Fehler in der Schichtdicke (insbesondere bei dickeren Schichten) resultiert daraus, dass für diese Schichten die Schichtdicke nicht mehr mittels Kleinwinkelröntgenreflektometrie bestimmt werden konnte. Die angegebenen Schichtdicken wurden aus der Menge der deponierten Lösung berechnet und aus den Ergebnissen der dünneren Schichten extrapoliert. Für die Schichten, in denen die Schichtdickenbestimmung nicht aus den Kleinwinkelröntgenreflektometrie möglich ist, wird ein Fehler von $d= \pm 10 \mathrm{~nm}$ angenommen.

Dickere Schichten zeigen eine größere, laterale Ausdehnung der einzelnen Körner. Der Einfluss dieser vergrößerten Körner auf den elektronischen Transport wird in Abb. 5.8 gezeigt. Bei steigender Schichtdicke bzw. größeren Korndurchmessern verschiebt sich die Metall-Isolator-Übergangstemperatur zu höheren Temperaturen. Auch $T_{\min }$ verändert sich als Funktion der Schichtdicke, je dicker die Schichten werden, desto kleiner wird $T_{\min }$.

\footnotetext{
${ }^{1}$ Die verschiedenen Körner zeigen sehr unterschiedliche geometrische Formen. Um die mittlere Ausdehnung eines Kornes bzw. eine „Korngröße“ angeben zu können, wird die geometrische Form durch einen Kreis genähert, wovon der Durchmesser als „Korngröße“ angegeben wird. Zusätzlich zu dem großen Fehler, der durch die Approximation einer Kreisform entsteht, wird eine vollständige Bedeckung der Fläche durch diese Kreise angenommen. Die angegebenen Korngrößen dienen daher lediglich dazu, das laterale Wachstum der Körner mit steigender Schichtdicke zu beschreiben.
} 


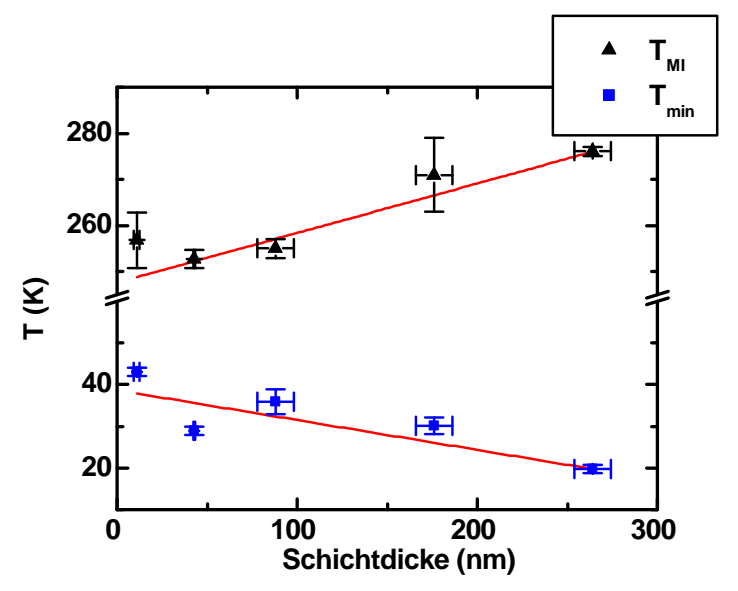

(a) $T_{\min }$ und $T_{\mathrm{MI}}$

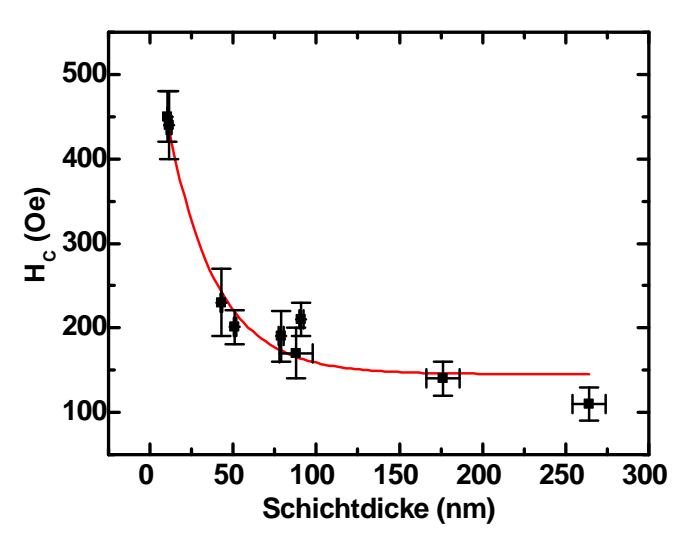

(b) Koerzitivfeld $H_{\mathrm{C}}$

Abb. 5.8: Einfluss der Schichtdicke in LSMO $/ \mathrm{Al}_{2} \mathrm{O}_{3}$ auf $T_{\mathrm{MI}}, T_{\min }$ und $H_{\mathrm{C}}$. (a) Bei steigender Schichtdicke nimmt $T_{\mathrm{MI}} \mathrm{zu}$ und $T_{\min }$ ab. Lineare Fits ergeben dabei $T_{\mathrm{MI}}(d)=(248 \pm 2 \mathrm{~K})+(0,11 \pm 0,01 \mathrm{~K} / \mathrm{nm}) \cdot d$ und $T_{\min }(d)=(38,7 \pm 4 \mathrm{~K})-(0,07 \pm$ $0,03 \mathrm{~K} / \mathrm{nm}) \cdot d$. (b) Das Koerzitivfeld nimmt als Funktion der Schichtdicke ab $\left(H_{\mathrm{C}}\right.$ bestimmt aus Magnetowiderstandsmessungen, $\left.T=10 \mathrm{~K}\right)$. Hier liefert ein exponentieller Fit $H_{\mathrm{C}}(d)=(430 \pm 54 \mathrm{Oe}) \cdot \exp [-d /(29 \pm 6 \mathrm{~nm})]+(145 \pm 15 \mathrm{Oe})$.

Zusätzlich sind in der Abb. 5.8(a) lineare Fits für die Funktionen $T_{\mathrm{MI}}(d)$ und $T_{\min }(d)$ eingetragen. In Abb. 5.8(b) ist die Abhängigkeit des Koerzitivfelds von der Schichtdicke mit einem exponentiellen Fit dargestellt, je größer die Schichtdicken werden, desto mehr nimmt das Koerzitivfeld ab.

\subsection{Diskussion}

Im nachfolgenden Abschnitt werden die Röntgen-, Rastertunnelmikroskopie-, elektronischen Transport- und Magnetisierungsmessungen der polykristallinen Manganatschichten diskutiert. Besondere Relevanz bei dieser Diskussion erhalten die Arbeiten von Esseling und Köster, da hier ebenfalls mittels MAD-Technik hergestellte LSMO/ $\mathrm{Al}_{2} \mathrm{O}_{3}$-Schichten untersucht wurden. [88, 89, 90]

\section{Kristallstruktur und Oberflächenmorphologie}

Die Ergebnisse der Röntgenmessungen wurden in Abschnitt 5.1 vorgestellt. Auf den MgOSubstraten findet sich nur die (001)-Orientierung der LSMO-Schicht, deren Gitterkonstante bei $c=3,877 \pm 0,006 \AA$ liegt. In Vorgängerarbeiten von Moshnyaga et al. und Raabe wurden mittels der MAD-Technik hergestellte LSMO/MgO Schichten charakterisiert, deren Gitterkonstanten mit $c=3,888 \AA$ bzw. $c=3,877 \AA$ angegeben werden. [71, 87] In diesen Arbeiten wurde das epitaktische Wachstum der Manganatschichten auf MgO-Substraten mittels TEM-Messungen bestätigt. Die erheblich unterschiedlichen Gitterkonstanten des MgO-Substrates $(c=4,211 \AA$ [91]) und der Manganatschichten werden durch Gitterfehlanpassungen innerhalb weniger Einheitszellen abgebaut. Dadurch wachsen die Schichten (nach diesen ersten Lagen) weitestgehend unverspannt auf. [71, 87] Entsprechend wird für die hier untersuchten LSMO/MgO-Proben ebenfalls von epitaktischem, unverspannten Wachstum ausgegangen. Dieses wird durch die Gitterkonstante bestätigt, die mit der von 


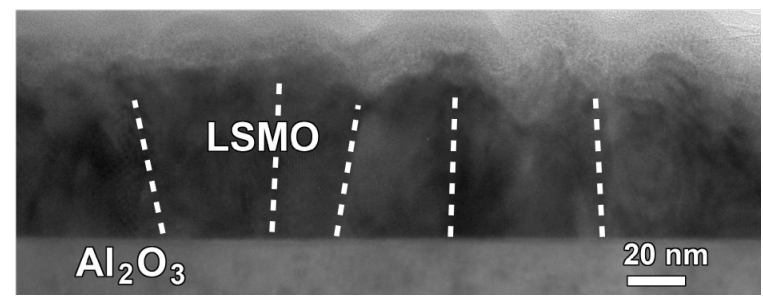

(a) cross section

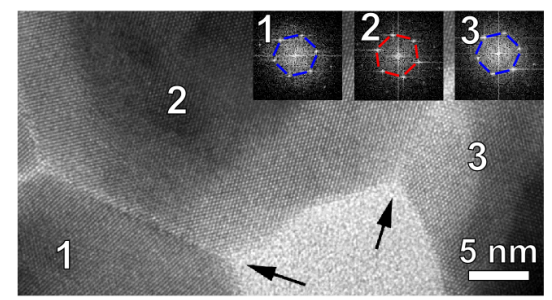

(b) plan view

Abb. 5.9: TEM-Messungen an LSMO/ $\mathrm{Al}_{2} \mathrm{O}_{3}$-Schichten von Lebedev, teilweise nach Ref. [93]. In (a) ist eine Querschnittsaufnahme (cross section) durch die Schicht und das Substrat gezeigt. In (b) ist eine hochauflösende Messung innerhalb der Schichtebene (plan view) abgebildet, in der zusätzlich die Beugungsbilder der einzelnen Körner eingezeichnet sind. Zu sehen ist das nanokolumnare Schichtwachstum in (a) und die Korngrenzen zwischen den um $30^{\circ}$ verdrehten (111)orientierten Körnern in (b).

Raabe und der von Haghiri-Gosnet et al. für Volumenproben angegebenen Gitterkonstante $(c=3,873 \AA$ [92]) übereinstimmt. Weiterhin wird das epitaktische Wachstum durch die STM-Aufnahmen (Abb. 5.3(b)) untermauert, die eine glatte Schichtoberfläche (RMS = $0,76 \mathrm{~nm})$ zeigen.

Während in den LSMO/MgO-Proben lediglich die (001)-Orientierung (in c-Richtung) beobachtet wird, zeigt das Röntgendiffraktogramm (Abb. 5.1(a)) für LSMO/ $\mathrm{Al}_{2} \mathrm{O}_{3}$-Schichten Intensitätsmaxima für die (001)-, (011)- und (111)-Orientierungen; die Schichten sind polykristallin. Für LSMO $/ \mathrm{Al}_{2} \mathrm{O}_{3}$ wird eine mittlere Gitterkonstante von $c=3,863 \pm 0,007 \AA$ ermittelt. Diese ist (im Rahmen der Fehlers) nur geringfügig kleiner $(0,4 \%)$ als die der epitaktischen Schicht und stimmt mit den Ergebnissen von Esseling überein $(c=3,86 \AA)$. [88]

TEM-Messungen der von Köster mittels MAD hergestellten LSMO $/ \mathrm{Al}_{2} \mathrm{O}_{3}$-Proben wurden von Lebedev (EMAT, University of Antwerp) durchgeführt, einige Ergebnisse dieser Studien sind in Abb. 5.9 zu finden (siehe auch Ref. [89, 90, 93]). Es wird ein nanokolumnares Schichtwachstum beobachtet, bei dem die Manganatkörner als Säulen von dem Substrat zur Schichtoberfläche wachsen (Abb. 5.9(a)) . Hochauflösende TEM-Messungen innerhalb der Schichtebene (plan view) sind in Abb. $5.9(\mathrm{~b})$ zu finden. In dieser Messung sind (111)orientierte Körner, die in der Schichtebene um $30^{\circ}$ zueinander verdreht sind, gezeigt. Durch die $\varphi$-Scans werden in dieser Arbeit ebenfalls um $30^{\circ}$ zueinander verdrehte, (111)-orientierte Körner nachgewiesen (Abb. 5.2). Dementsprechend wird ebenfalls nanokolumnares Wachstum angenommen. Dieses wird durch die Morphologieaufnahmen aus REM-, STMund AFM-Messungen, welche die gleiche Morphologie wie die von Esseling und Köster präparierten und mittels TEM untersuchten Schichten zeigen, bestätigt. [88, 89] Ebenso wie in den Ergebnissen von Esseling sind in Abb. 5.3(a) die (111)-orientierten Körner als Dreiecke zu erkennen.

\section{Elektronische und magnetische Struktur}

Der Einfluss der Mikrostruktur in den LSMO $/ \mathrm{Al}_{2} \mathrm{O}_{3}$-Schichten auf die elektronischen und magnetischen Eigenschaften wird durch einen Vergleich mit den epitaktischen LSMO/MgOSchichten deutlich. In Abb. 5.5 ist die Temperaturabhängigkeit des elektrischen Widerstands und der Magnetisierung der Schichten gezeigt. In dieser Abbildung werden zwei 
Schichten auf unterschiedlichen Substraten gegenübergestellt, die während des gleichen Depositionsprozesses hergestellt wurden. Die Schichtdicken (bestimmt aus den Kleinwinkelröntgenreflektometrie) sind im Rahmen der Messgenauigkeit gleich $(d=42 \ldots 43 \mathrm{~nm})$. Es kann entsprechend von einer identischen Dotierung ausgegangen werden. Unterschiede der elektronischen und magnetischen Eigenschaften sind daher auf den Einfluss der Mikrostruktur zurückzuführen.

Die Curie-Temperatur der zwei Schichten verändert sich wesentlich weniger als die Metall-Isolator-Übergangstemperatur: Für die LSMO/ $/ \mathrm{Al}_{2} \mathrm{O}_{3}$-Probe wird $T_{\mathrm{C}}=357 \pm 2 \mathrm{~K}$ und $T_{\mathrm{MI}}=253 \pm 1 \mathrm{~K}$, für LSMO $/ \mathrm{MgO} T_{\mathrm{C}}=352 \pm 2 \mathrm{~K}$ und $T_{\mathrm{MI}}=382 \pm 1 \mathrm{~K}$ ermittelt. Während Magnetisierungsmessungen das Schichtvolumen untersuchen, wird das elektronische Transportverhalten von den (hochohmigen) Korngrenzen dominiert. Die nur leicht veränderte Curie-Temperatur bedeutet entsprechend, dass der größte Volumenanteil der Schicht durch die Grenzflächen nur geringfügig beeinflusst wird. Dies spiegelt sich auch in der nur leicht veränderten Gitterkonstante wieder. Der elektronische Transport dagegen ist in den polykristallinen Schichten durch die Korngrenzen stark verändert. Neben der MetallIsolator-Übergangstemperatur unterscheidet sich auch der spezifische Widerstand stark: Zwischen dem spezifischen Widerstand der epitaktischen und polykristallinen Schichten liegen fast drei Größenordnungen. Zusätzlich findet sich in den polykristallinen Schichten ein Widerstandsanstieg bei tieferen Temperaturen.

In den polykristallinen Schichten wird zudem ein Kleinfeldmagnetowiderstand (LFMR) beobachtet, der in den epitaktischen Schichten nicht auftritt (Abb. 5.6). Der LFMR wird in der Fachliteratur durch das spinpolarisierte Tunneln von Ladungsträgern zwischen den verschiedenen Körnern (über die isolierenden Korngrenzen) erklärt. [94, 95, 96, 97] Für den Widerstand ist die relative Orientierung der Magnetisierung von benachbarten Körnern entscheidend. Entsprechend zeigt ein Vergleich der $\mathrm{R}(\mathrm{H})$-Kurven mit den magnetischen Hysteresekurven (ebenfalls in Abb. 5.6), dass die Widerstandsmaxima bei $M=0$ und $H=$ $H_{\mathrm{C}}$ liegen, also in dem Bereich, in welchem keine parallele Ausrichtung der Orientierung der Magnetisierung benachbarter Körner vorhanden ist.

Für LSMO $/ \mathrm{Al}_{2} \mathrm{O}_{3}$ wird ein Koerzitivfeld von $H_{\mathrm{C}}=230 \pm 10$ Oe und für LSMO/MgO von $H_{\mathrm{C}}=140 \pm 10$ Oe ermittelt. Des Weiteren nimmt das Koerzitivfeld als Funktion der Schichtdicke bzw. des mittleren Korndurchmessers ab (Abb. 5.8(b)). Hierbei wird sogar $H_{\mathrm{C}}=110 \pm 20$ Oe für eine $264 \pm 10 \mathrm{~nm}$ dicke Schicht erreicht. Fontcuberta und Balcells et al. beobachten ebenfalls eine Reduzierung von $H_{\mathrm{C}}$ als Funktion des mittleren Korndurchmessers für $20 \mathrm{~nm} \ldots 75 \mu \mathrm{m}$ große $\mathrm{La}_{2 / 3} \mathrm{Sr}_{1 / 3} \mathrm{MnO}_{3}$-Körner. Sie schließen hieraus, dass in den Körnern nur eine Domäne vorhanden ist. [95, 98] Auch Untersuchungen von Steren et al. an $\mathrm{La}_{0,6} \mathrm{Sr}_{0,4} \mathrm{MnO}_{3}$-Schichten auf $\mathrm{MgO}$ - und $\mathrm{SrTiO}_{3}$-Substraten mit unterschiedlichen Schichtdicken $d=5 \ldots 500 \mathrm{~nm}$ zeigen eine systematische Abnahme des Koerzitivfeldes und werden aufgrund einer veränderten Domänenstruktur durch vergrößerte Pinning-Effekte in den dünneren Schichten erklärt. [99]

Um das veränderte elektronische Transportverhalten zu verstehen, wurden vielfach Untersuchungen an polykristallinen Manganatproben mit unterschiedlichen Korngrößen durchgeführt. [95, 100] Hierbei wird ebenfalls (entsprechend der Messungen in Abb. 5.8(a)) eine systematische Veränderung von $T_{\mathrm{MI}}$ als Funktion der mittleren Korngröße beobachtet: Je größer die Korndurchmesser werden, desto höher wird $T_{\mathrm{MI}}$, wobei sich $T_{\mathrm{C}}$ allerdings kaum verändert. [101, 102, 103 ]

Zur Erklärung dieser Ergebnisse wurde das core shell-Modell eingeführt. [98, 100, 101, 104 ] Im Rahmen dieses Modells werden der Kornhülle (shell) und dem Korninneren (core) un- 
terschiedliche physikalische Eigenschaften zugeschrieben. Das Korninnere verhält sich dabei wie bulk-Proben oder im hier untersuchten Fall wie die epitaktische Schicht. Dieses wird zum Beispiel in der nur kaum veränderten Curie-Temperatur deutlich. Die Oberfläche der Körner dagegen zeigt veränderte magnetische und elektronische Eigenschaften, was in den veränderten elektronischen Transporteigenschaften deutlich wird. ${ }^{2}$ Zudem wird berichtet, dass die Kornhülle eine Funktion des mittleren Korndurchmessers ist und bei steigendem Korndurchmesser dünner wird. [98, 104, 105]

Esseling findet bei einer $70 \mathrm{~nm}$ dicken $\mathrm{LSMO} / \mathrm{Al}_{2} \mathrm{O}_{3}$-Schicht $T_{\mathrm{C}}=375 \mathrm{~K}$ und $T_{\mathrm{MI}}=$ $365 \mathrm{~K}$. [88] In Abb. 5.8 ist die Metall-Isolator-Übergangstemperatur als Funktion der Schichtdicke gezeigt, selbst für eine $264 \pm 10 \mathrm{~nm}$ dicke Schicht erhöht sich $T_{\mathrm{MI}}$ nur auf $276 \pm 1 \mathrm{~K}$. Durch eine unterschiedliche Schichtdicke kann daher die höhere Metall-Isolator-Übergangstemperatur von Esseling nicht erklärt werden. Die Unterschiede der Proben sind vermutlich durch unterschiedliche Depositionsbedingungen zu erklären: Insbesondere die Depositionsrate war für die Herstellung der Schichten sehr unterschiedlich. Während Esseling eine Rate von etwa $1 \mathrm{~nm} / \mathrm{s}$ verwendet hat, wurden die Schichten in dieser Arbeit mit einer deutlich geringeren Rate hergestellt $(\approx 0,4 \mathrm{~nm} / \mathrm{s})$.

In den nanokolumnaren Schichten wird zusätzlich bei tiefen Temperaturen ein Widerstandsanstieg $\left(T_{\min }\right)$ beobachtet, ${ }^{3}$ siehe Abb. 5.5(a) und 5.8(a), der bei den epitaktischen Proben (bei optimalen Depositionsbedingungen) nicht beobachtet wird. Bei größeren Schichtdicken bzw. Korngrößen verändert sich $T_{\min }$ zu höheren Temperaturen, siehe Abb. 5.8(a). Dieses Verhalten wird ebenfalls vielfach in der Literatur beobachtet und einer Coulomb-Blockade zugeschrieben. [95, 98, 101]

\subsection{Zusammenfassung}

In diesem Kapitel wurde eine Charakterisierung von $\mathrm{La}_{0,7} \mathrm{Sr}_{0,3} \mathrm{MnO}_{3} / \mathrm{Al}_{2} \mathrm{O}_{3}$-Proben aufgrund ihrer strukturellen, elektronischen und magnetischen Eigenschaften vorgestellt. Diese Schichten zeigen polykristallines, nanokolumnares Wachstum, wobei unterschiedliche Orientierungen auftreten. Ein Vergleich zu den epitaktischen LSMO/MgO-Schichten zeigt, dass die Gitterkonstante kaum verändert ist. Ebenso ist die Curie-Temperatur bei zwei gleichzeitig deponierten Schichten nur geringfügig verändert. Im Gegensatz dazu zeigen die Korngrenzen einen großen Einfluss auf den elektronischen Transport: Die Metall-Isolator-Übergangstemperatur ist in den polykristallinen Schichten erniedrigt, der spezifische Widerstand erhöht. Zusätzlich tritt ein Widerstandsminimum bei tiefen Temperaturen auf und es wird ein LFMR beobachtet. Die Ergebnisse der elektronischen und magnetischen Messungen können gut im Rahmen von spinpolariziertem Tunneln zwischen den Körnern erklärt werden, wobei ein Unterschied der physikalischen Eigenschaften zwischen der Kornhülle und dem Korninneren angenommen wird (core shell-Modell). Auch die Ergebnisse der Schichtdickenserie stimmen gut mit der Literatur zu diesem Modell überein.

\footnotetext{
${ }^{2}$ Balcells et al. geben für die Hülle eine Dicke von 1,2 $\mathrm{nm}$ an (Korndurchmesser $20 \mathrm{~nm}$ ). 105

${ }^{3}$ Dieser Widerstandsanstieg wird von Esseling in einer $70 \mathrm{~nm}$ dicken $\mathrm{LSMO} / \mathrm{Al}_{2} \mathrm{O}_{3}$-Schicht bei $T_{\min }=40 \mathrm{~K}$ gefunden. [88 Im Vergleich dazu wird in dieser Arbeit für eine $88 \pm 10 \mathrm{~nm}$ dicke Schicht $T_{\min }=36 \pm 3 \mathrm{~K}$ gemessen.
} 


\section{Elektrisches Widerstandsschalten in nanokolumnaren Manganatschichten}

Bei dem bipolaren Widerstandsschalten können unterschiedliche remanente Widerstandszustände durch elektrische Felder variierender Polarität eingestellt werden. In diesem Kapitel wird das Widerstandsschalten in nanokolumnaren Manganatschichten untersucht. Dabei handelt es sich um $\mathrm{La}_{0,7} \mathrm{Sr}_{0,3} \mathrm{MnO}_{3}$-Schichten (LSMO), die auf $\mathrm{Al}_{2} \mathrm{O}_{3}$-Substraten deponiert wurden, wie im Kap. 5 beschrieben wird.

Die in diesem Kapitel vorgestellten Messergebnisse sind nach den verwendeten Methoden gegliedert: Als erstes werden die Schaltexperimente mittels C-AFM vorgestellt. Mit dem C-AFM kann das Widerstandsschalten auf der Nanometerskala - und damit auf der Längenskala der nanokolumnaren Manganatsäulen - analysiert werden. Im Abschnitt 6.2 folgt die Untersuchung des Effekts in den mittels Elektronenstrahl-Lithographie strukturierten Manganatproben. In diesen wird das globale bzw. gemittelte Verhalten vieler Manganatsäulen charakterisiert, da die Strukturgrößen im $\mu \mathrm{m}$-Bereich liegen, während die Korndurchmesser zwischen $40 \ldots 130 \mathrm{~nm}$ variieren.

Abschnitt 6.3 erörtert anschließend diese Messergebnisse. Hierbei wird der Fokus auf den Einfluss der Grenzflächen (hier Manganatoberflächen und Korngrenzen) gelegt. Das elektrisch induzierte Schalten wird zunächst anhand einer verallgemeinerten Energielandschaft eingeordnet. Die Mikrostrukturmessungen werden im Rahmen eines Widerstandsnetzwerkes simuliert, um schließlich den zugrundliegenden mikroskopischen Mechanismus zu diskutieren.

\subsection{C-AFM Messungen}

Die gezeigten C-AFM Schaltexperimente wurden in Zusammenarbeit mit J.-O. Krisponeit durchgeführt, der im Rahmen seiner Doktorarbeit [60] eine Charakterisierung des bipolaren Widerstandsschaltens an epitaktischen Manganatschichten durchgeführt hat. Die C-AFM Messergebnisse von epitaktischen und nanokolumnaren Manganatschichten zeigen viele Parallelen, wobei in dieser Arbeit der Fokus auf die Besonderheiten der nanokolumnaren Schichten gelegt wird.

Für die epitaktischen Manganatschichten wurde gezeigt, dass der Anfangs- bzw. Ausgangszustand stets hochohmig ist (HRS: high resistive state). [60] Dieser Anfangszustand kann 
lokal durch das Anlegen einer positiven Schaltspannung von mindestens $U_{\mathrm{c}}=+3 \mathrm{~V}$ in einen niederohmigeren Zustand (LRS: low resistive state) geschaltet werden. ${ }^{1}$ Wird eine (ausreichend große) negative Spannung angelegt, so kann der HRS wieder hergestellt werden. Dieser Effekt ist reversibel, das bedeutet, dass wiederholt zwischen HRS und LRS geschaltet werden kann.

In den C-AFM Messungen der nanokolumnaren Schichten wird ebenfalls dieses Verhalten beobachtet: Bei einer ausreichend großen positiven Spannung kann der zu Anfang vorliegende HRS in den LRS geschaltet werden, entsprechend wird bei einer negativen Schaltspannung der HRS wiederhergestellt. Die kritische Schaltspannung $U_{\mathrm{c}}$ ist in den nanokolumnaren Schichten allerdings niedriger als in den epitaktischen Proben, dieses wird im Abschnitt 6.1.2 demonstriert.

Die Widerstandsänderungen zwischen HRS und LRS können über

$$
\mathrm{EPIR}=\left(\frac{R_{\mathrm{HRS}}-R_{\mathrm{LRS}}}{R_{\mathrm{LRS}}}\right) \cdot 100 \%
$$

quantifiziert werden. EPIR steht hier für die englische Abkürzung von electric pulse induced resistance, wie dieser Effekt in der Literatur oft bezeichnet wird. Für die nanokolumnaren Schichten ergeben sich Widerstandsänderungen von EPIR $\approx 26000 \%$. Dieser Wert wird durch einen Fit in dem Bereich kleiner Spannungen der I(U)-Kennlinien bestimmt, wobei sich die Widerstandswerte $R_{\mathrm{HRS}}=615 \mathrm{M} \Omega$ und $R_{\mathrm{LRS}}=2,34 \mathrm{M} \Omega$ ergeben. Die I(U)Kennlinien von HRS und LRS werden an dieser Stelle nicht gezeigt, da kein qualitativer Unterschied zu den Ergebnissen von Ref. [60] beobachtet wird.

In den nachfolgenden Abschnitten 6.1.1 und 6.1.2 wird die Verteilung der mittels C-AFM induzierten LRS-Bereiche mit der Topographie verglichen. Die Untersuchungen stammen dabei von Messungen an einer $44 \mathrm{~nm}$ dünnen LSMO-Schicht. Im Unterkapitel 6.1.3 werden die Messergebnisse an einer dickeren Schicht $(d=300 \mathrm{~nm})$ vorgestellt.

In den C-AFM Experimenten wurden viele wichtige Messparameter im Laufe der Messungen nicht verändert. Dazu zählt, dass alle gezeigten C-AFM Messungen bei Raumtemperatur in dem im Abschnitt 4.7 beschriebenen UHV-Mikroskop aufgenommen wurden. Des Weiteren werden die Messungen im Kontakt-Modus durchgeführt. Dabei ist die langsame Scanrichtung in den Abbildungen stets von unten nach oben gerichtet, wie der Pfeil in Abb. 6.1(a) verdeutlicht. Zum Verständnis der Ergebnisse ist es auch wichtig zu beachten, dass das Substrat der untersuchten Schichten isolierend ist $\left(\mathrm{Al}_{2} \mathrm{O}_{3}\right)$. Die Proben sind an den Rändern großflächig mit Leitsilber kontaktiert; der Strom fließt also von der Cantileverspitze durch die Manganatschicht zu diesen äußeren, makroskopischen Kontakten. Das bedeutet, dass ein planarer Messaufbau vorliegt, wie er im Kap. 3 in Abb. 3.1(b) beschrieben wird.

\subsubsection{Räumliche Anordnung von HRS und LRS}

In Abb. 6.1 sind C-AFM Messungen gezeigt, in denen der zu Anfang vorliegende, hochohmige Zustand in einigen Bereichen in den LRS geschaltet wird. Bei der folgenden Beschreibung der Ergebnisse wird zwischen einer Messspannung $U_{\text {mess }}$ und einer Schaltspannung $U_{\text {schalt }}$ unterschieden: $U_{\text {mess }}$ entspricht dabei einer kleinen Spannung $\left(U_{\text {mess }}<1 \mathrm{~V}\right)$. Die Einteilung der Schicht in HRS und LRS erfolgt nach der Größe des Stromflusses bei anliegender Messspannung. Die Schaltspannung $U_{\text {schalt }}$ ist typischerweise einige Volt groß

\footnotetext{
${ }^{1}$ Die angegebenen Polaritäten beziehen sich auf die an der Cantileverspitze anliegende Spannungspolarität.
} 


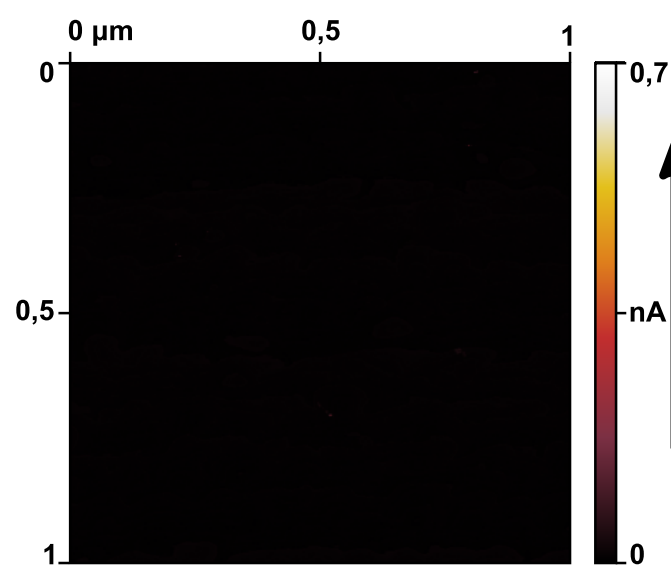

(a) Stromkarte I

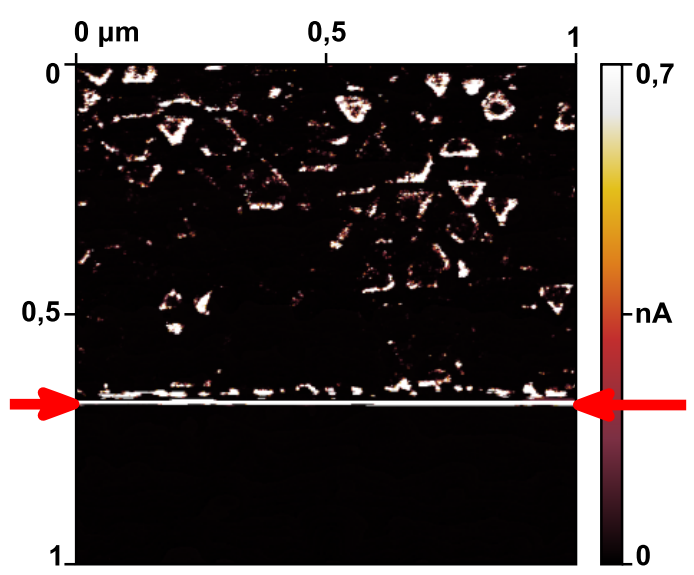

(c) Stromkarte II

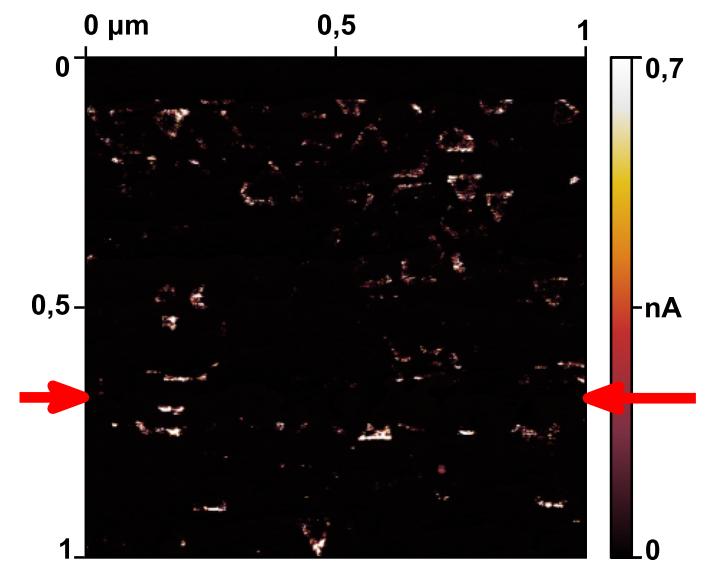

(e) Stromkarte III

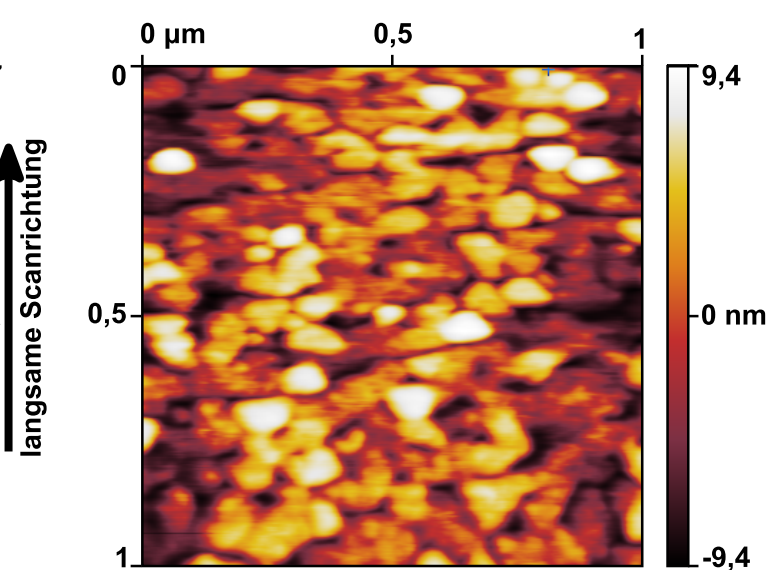

(b) Topographie I

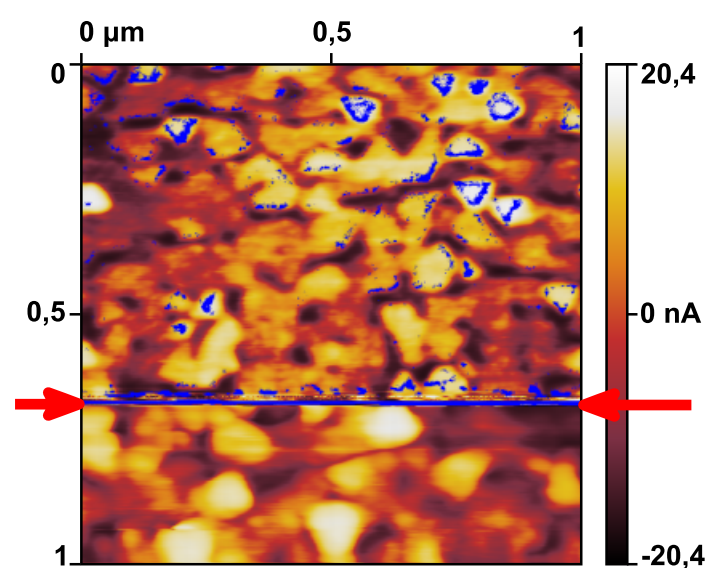

(d) Topographie II (in Blau: Stromkarte)

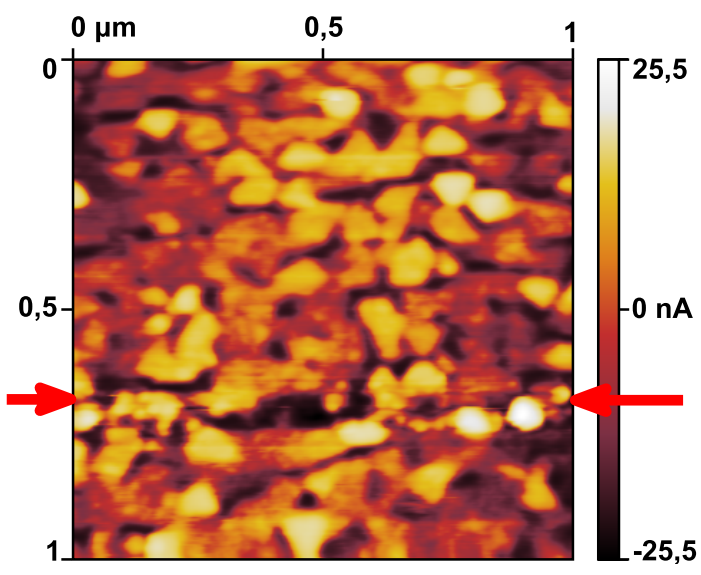

(f) Topographie III

Abb. 6.1: Räumliche Anordnung von HRS und LRS: Messung I zeigt den Ausgangszustand der Schicht, in (a) die Stromkarte und in (b) die simultan aufgenommene Topographie $\left(U_{\text {mess }}=0,1 \mathrm{~V}\right)$. In Messung II $((\mathrm{c})$ und $(\mathrm{d}))$ wird die Spannung in dem mit den roten Pfeilen gekennzeichneten Bereich während einiger Scanlinien auf $U_{\text {schalt }}=5 \mathrm{~V}$ erhöht. Danach beträgt $U_{\text {mess }}=0,5 \mathrm{~V}$. Nach dem Anlegen von $U_{\text {schalt }}$ sind einige Probenbereiche in den LRS geschaltet, wie in Messung II (c) und (d) (oberhalb der roten Pfeile) und in Messung III (e) und (f) zu sehen ist. 
und wird für eine begrenzte Zeitdauer (hier $\tau=$ ms...s) angelegt, um das Widerstandsschalten zu initiieren.

Der Ausgangszustand ${ }^{2}$ der nanokolumnaren Manganatschicht ist in Abb. 6.1(a) gezeigt. Bei einer Spannung von $U_{\text {mess }}=0,1 \mathrm{~V}$ ist der Stromfluss kleiner als $0,1 \mathrm{nA}$ (dieses gilt für 99,998\% der Messpunkte). Dieser Ausgangszustand wird dementsprechend als homogen isolierend (HRS) charakterisiert.

In Abb. 6.1(c) (Messung II) handelt es sich um eine C-AFM Messung auf dem selben Bereich auf der Probe. Dieses ist auch in den jeweiligen Topographien deutlich zu erkennen (Abb. 6.1(b) und 6.1(d)). Während der Aufnahme von Messung II wird die Spannung für einige Scanlinien (diese sind mit roten Pfeilen gekennzeichnet) auf $U_{\text {schalt }}=5 \mathrm{~V}$ erhöht. Danach wird auf eine Messspannung von $U_{\text {mess }}=0,5 \mathrm{~V}$ reduziert. Da die langsame Scanrichtung von unten nach oben gerichtet ist (wie der Pfeil in Abb. 6.1(a) verdeutlicht), wurden die Linien unterhalb der roten Pfeile vor dem Anlegen von $U_{\text {schalt }}$ aufgenommen (also im Ausgangszustand), der Bereich oberhalb danach. Die Stromkarte zeigt, dass nach dem Anlegen der Schaltspannung bzw. oberhalb der roten Pfeile einige Probenbereiche in den LRS geschaltet sind (bei $U_{\text {mess }}=0,5 \mathrm{~V}$ ist $I>0,7 \mathrm{nA}$ ).

In Abb. 6.1(d) ist die zu der Stromkarte II zugehörige Topographie dargestellt. Um die Korrelation zwischen der Topographie und der Verteilung der LRS-Bereiche zu untersuchen, ist die Topographie mit der Stromkarte überlagert. ${ }^{3}$ In dieser Abbildung ist zu erkennen, dass in unmittelbarer Nähe der mit $U_{\text {schalt }}$ gescannten Linien (bis zu einem Abstand von $\approx 40 \mathrm{~nm}$ ) ein großer Anteil an LRS zu finden ist. Hier kann kein direkter Zusammenhang zwischen der lokalen Topographie und der räumlichen Anordnung des LRS beobachtet werden. In einem größeren Abstand zeigt sich eine eindeutige Korrelation zwischen der Topographie und der Verteilung der LRS-Bereiche: Der LRS-Zustand findet sich bevorzugt in den Randbereichen der nanokolumnaren Säulen. In der Stromkarte in Abb. 6.1(c) sind insbesondere LRS-Dreiecke und -Sechsecke zu beobachten. In diesen ist allerdings der mittlere Bereich unverändert isolierend. Der maximale Abstand zwischen den mit $U_{\text {schalt }}$ gerasterten Linien und der oberen Kante des Scanbereiches, in dem es LRS-Bereiche gibt, beträgt $650 \mathrm{~nm}$.

Zur quantitativen Analyse der Übereinstimmung der LRS-Bereiche mit dem Bereich der Korngrenzen wurde wie folgt vorgegangen. Aus der Topographie wird mittels des SobelFilters ein Gradientenbild erzeugt, wie in Abb. 6.2(b) gezeigt wird. In diesem Gradientenbild werden alle Bereiche oberhalb einer Schwelle $S$ markiert (siehe Abb. 6.2(c)). Die Übereinstimmung der markierten Bereiche mit der Stromkarte wird als quantitativer Massstab für die Übereinstimmung der LRS-Bereiche mit den Korngrenzen (Gebieten großer Steigung) genommen. Dabei wird der prozentuale Anteil der LRS-Bereiche, die innerhalb der Schwellwert markierten Gebiete liegen, bestimmt. Die erhaltenen Werte sind sehr stark abhängig von der Wahl der Schwellwertmarkierung $S$ in dem Gradientenbild und der Zuordnung zu einem LRS-Bereich $\left(I>I_{k}\right)$.

Diese Auswertung wird getrennt für die in Abb. 6.2(e) gekennzeichneten Bildausschnitte I und II durchgeführt. In Bildausschnitt I ergibt sich für eine Schwellwertmarkierung von $S=33 \%$ der Fläche und einer LRS-Zuordnung der Bereiche für $I>0,2 \mathrm{nA}$ eine Übereinstimmung von $60 \%$. Wird $I>40 \mathrm{nA}$ gewählt, so liegt die Übereinstimmung sogar bei

\footnotetext{
${ }^{2}$ Wenn innerhalb eines Messbereichs noch keine größere Spannung als die Messspannung angelegt wurde, wird dieser Probenbereich als „im Ausgangszustand“ bezeichnet.

${ }^{3}$ In Abb. 6.1(d) ist gleichzeitig die Topographie und zugehörige Stromkarte gezeigt. Für diese Darstellung wird die Stromkarte (Abb. 6.1(c) in HRS und LRS eingeteilt: Alle Bereiche mit einem Stromfluss $I>0,7 \mathrm{nA}$ werden dem LRS zugeordnet und in der Topographie in Blau eingetragen.
} 
(a) Topographie

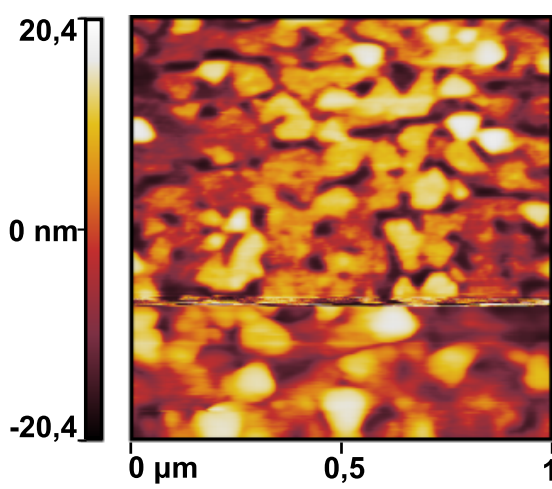

(d) Stromkarte

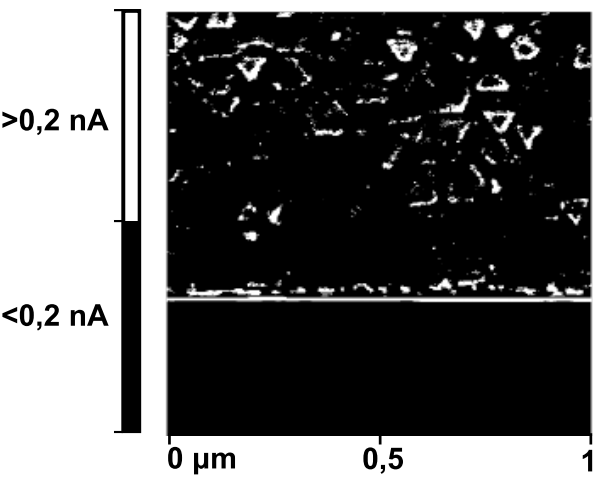

(b) Gradientenbild (Sobel)

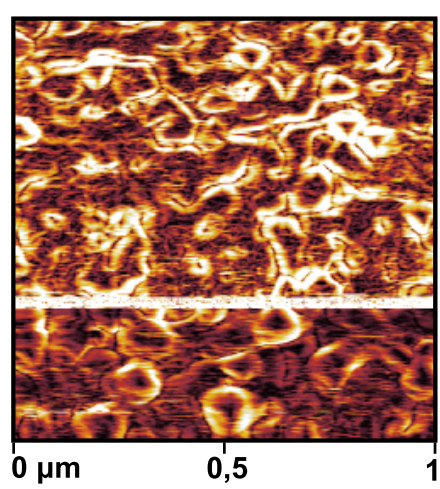

(c) Gradientenbild, Schwellwert in Blau markiert

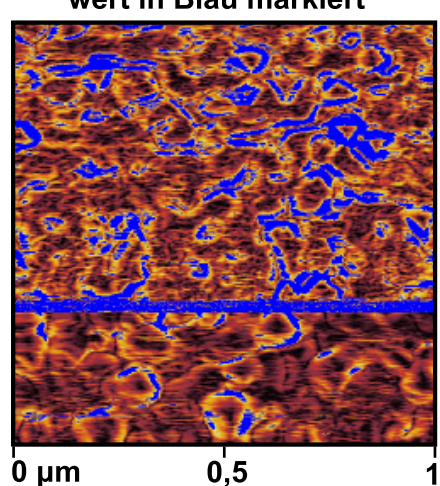

(e) Stromkarte und Sobel

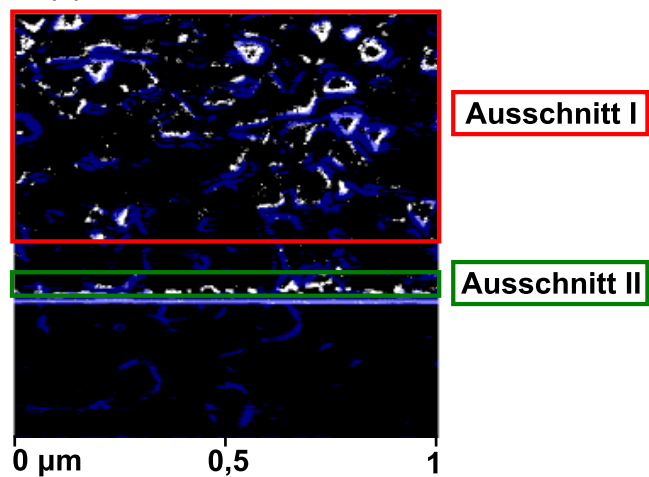

Abb. 6.2: Quantitative Analyse der Übereinstimmung zwischen Topographie und Stromkarte. Mittels eines Sobel-Filters wird ein Gradientenbild (b) der Topographie (a) erzeugt und anschließend alle Bereiche des Gradientenbildes oberhalb eines Schwellwertes markiert (c). Die Übereinstimmung der markierten Bereiche mit den LRS-Bereichen aus der Stromkarte wird als Massstab für die Übereinstimmung genommen.

66,7\%. Im Bildausschnitt II (in unmittelbarer Nähe der geschalteten Linie) dagegen ist die Übereinstimmung bei $S=27 \%$ für $I>0,2 \mathrm{nA}$ nur $40,6 \%$ und bei $I>40 \mathrm{nA}$ ist sie $50 \%$. Die Übereinstimmung der Bereiche großer Steigung ist also weiter entfernt von den mit $U_{\text {schalt }}$ gerasterten Linien wesentlich größer als in unmittelbarer Nähe dazu.

In Abb. 6.3(a) ist ein vergrößerter Bildausschnitt aus Abb. 6.1(d) abgebildet. Aus diesem Bereich ist in Abb. 6.3(b) ein Linienprofil der Stromkarte und der Topographie gezeigt. In dem Höhenprofil ist ebenfalls zu sehen, dass die LRS-Bereiche (meist) an den Flanken von Erhebungen, also im Bereich der Korngrenzen, zu finden sind. Die Breite der LRSBereiche wird bestimmt, in dem im $90^{\circ}$-Winkel entlang der Kanten der Körner die Länge ausgemessen wird. Es ergibt sich eine mittlere Breite von $d=11,2 \pm 0,5 \mathrm{~nm}$.

Bei der Interpretation dieser Messungen muss allerdings beachtet werden, dass die Spitze des Cantilevers einen sphärischen Spitzenradius von $30 \ldots 40 \mathrm{~nm}$ hat. Damit liegt sie in der Größenordnung der zu untersuchenden Manganatsäulen. Die Aufösung der Messung ist daher nicht ausreichend, um die LRS-Bereiche genau zu analysieren: Befinden sich zwischen den (in der Topographie dargestellten) großen Körnern kleinere, so können diese möglicherweise nicht abgebildet werden. Daher kann nicht unterschieden werden, ob die Korngrenze selbst hochohmig ist und von zwei LRS-Bereichen umgeben ist, oder ob die 


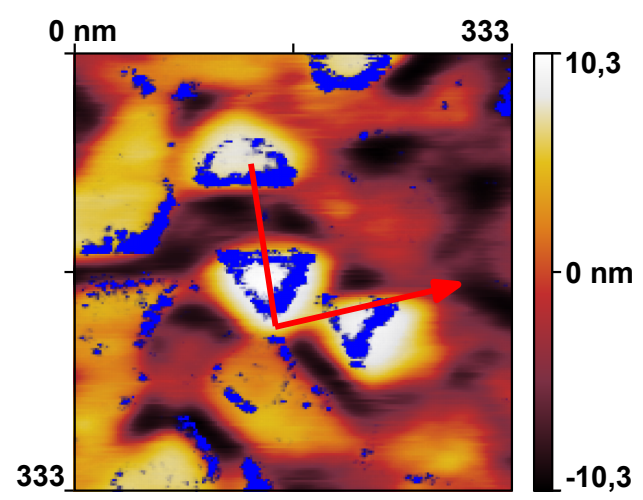

(a) Topographie mit überlagerter Stromkarte

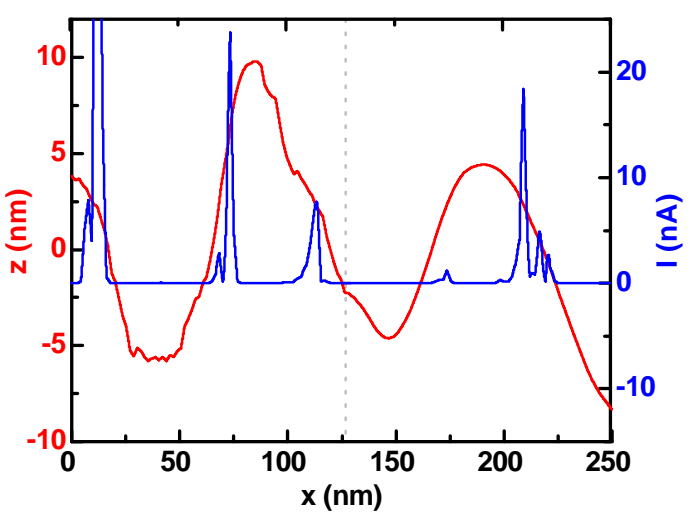

(b) Linienprofil aus Topographie und Stromkarte

Abb. 6.3: Linienprofil aus Topographie und Stromkarte: In (a) ist ein vergrößerter Ausschnitt aus Abb. 6.1(d) gezeigt. Der rote Pfeil zeigt die Position der Linienprofile in (b) an.

Korngrenze auch in den LRS schaltet. Als Ergebnis der C-AFM Messungen kann daher lediglich festgehalten werden, dass das Widerstandsschalten im Bereich der Korngrenzen stattfindet; eine genauere Unterscheidung ist aufgrund der Größe der Cantileverspitze nicht möglich.

Messung III (Abb. 6.1(e) und (f)) wurde im Anschluss an Messung II aufgenommen. Die Messspannung beträgt weiterhin $U_{\text {mess }}=0,5 \mathrm{~V}$. In der Stromkarte ist zu sehen, dass auch Bereiche unterhalb der durch die roten Pfeile gekennzeichneten Linien im LRS-Zustand sind. Daher kann - auf dieser Längenskala - kein eindeutiger Zusammenhang zwischen der Lage der makroskopischen Kontakte und der Ausrichtung der geschalteten Bereiche beobachtet werden. Ein Vergleich mit Messung II zeigt, dass einige LRS-Bereiche (oberhalb der roten Pfeile) in Messung III kleiner als in Messung II sind. Dieses Schrumpfen der leitfähigen Bereiche wird auch bei den epitaktischen Proben beobachtet [60] und wird daher an dieser Stelle nicht weiter analysiert. In der Topographie III ist weiterhin zu erkennen, dass sich die lokale Morphologie im Bereich der Linien, die mit der Schaltspannung aufgenommen worden sind, verändert hat. Auch diese Beobachtung wird in der Arbeit von Krisponeit [60] charakterisiert und deswegen hier nicht untersucht.

\subsubsection{Wachstum der LRS-Bereiche}

Um Bereiche der Probe in den LRS zu schalten, müssen nicht zwingend mehrere Linien mit der erhöhten Schaltspannung abgerastert werden. Es ist ausreichend, einzelne Spannungspulse anzulegen. Dadurch können Zwischenstufen zwischen dem HRS-Ausgangszustand und den komplett umgeschalteten LRS-Korngrenzen untersucht werden. Dazu wird zwischen einer Serie von Spannungspulsen immer wieder die Stromkarte und die Topographie aufgenommen. Die Entstehung der leitfähigen Bereiche wird somit als Funktion der Anzahl von Spannungspulsen charakterisiert. Hierzu gibt es auch Untersuchungen an epitaktischen Manganatschichten von Krisponeit. [60]

Eine Messserie, die Stromkarten nach einer unterschiedlichen Anzahl von positiven Spannungspulsen zeigt, ist in Abb. 6.4 abgebildet. Schon in der ersten Messung (vor dem ersten 

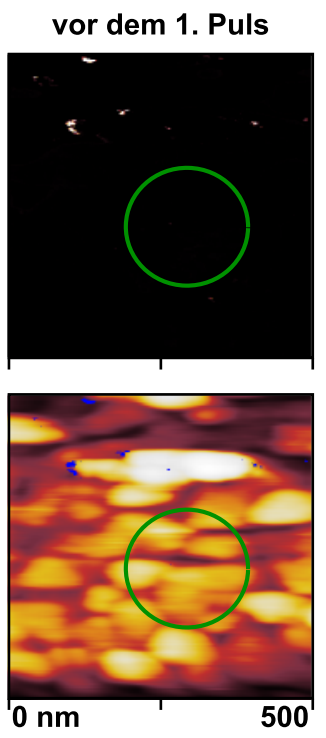

1. Puls
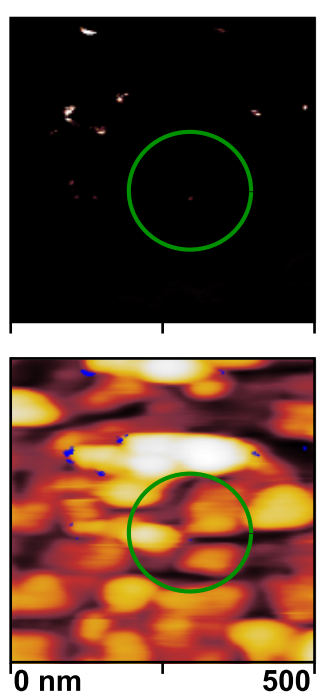

2. Puls
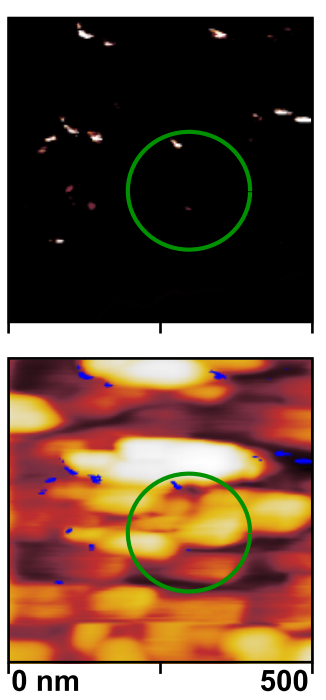

7. Puls
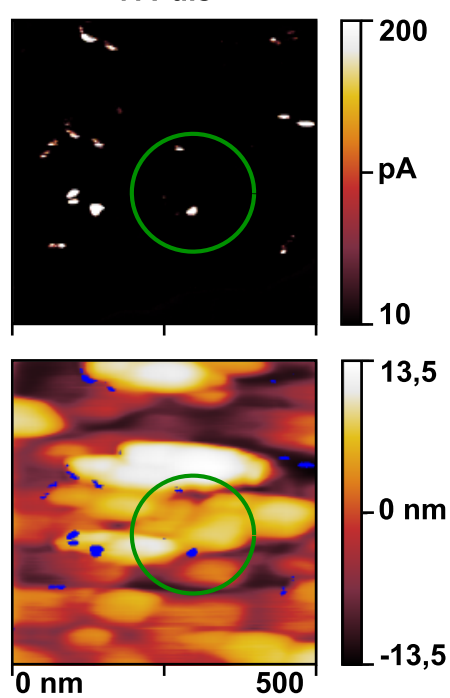

Abb. 6.4: C-AFM Messungen nach einer unterschiedlichen Anzahl von Spannungspulsen $\left(U_{\text {schalt }}=8 \mathrm{~V}\right.$, Pulsdauer $\left.\tau=10 \mathrm{~ms}, U_{\text {mess }}=0,1 \mathrm{~V}\right)$. Der grüne Kreis markiert den Bereich, in dem die Pulse gesetzt worden sind. In der oberen Zeile sind die Stromkarten gezeigt, in der unteren die zugehörigen Topographien. Durch die Pulse verändert sich die Größe und Anzahl der LRS-Gebiete im Bereich der Korngrenzen.

Puls) finden sich einige wenige Bereiche, in denen ein tiefohmiger Zustand existiert. Für die in Abb. 6.4 gezeigten Stromkarten wird allerdings eine veränderte Skala, die nur bis 200 pA reicht, verwendet. Für die gemeinsame Darstellung der Topographie und Stromkarte werden alle Werte oberhalb dieser Schwelle dem LRS zugeordnet $\left(U_{\text {mess }}=0,1 \mathrm{~V}\right)$.

Die zu Anfang vorliegenden LRS-Bereiche befinden sich an den Rändern der Körner, wie ein Vergleich zu der Topographie zeigt. Diese LRS-Bereiche wurden möglicherweise durch Schaltspannungen aus vorherigen Messungen verursacht. Nach dem ersten Puls $\left(U_{\text {schalt }}=\right.$ $8 \mathrm{~V}$, Pulsdauer $\tau=10 \mathrm{~ms}$ ) hat die Zahl der leitfähigen Bereiche zugenommen bzw. die LRS-Bereiche haben sich vergrößert. Auch weitere Pulse vergrößern die Anzahl und Größe der LRS-Bereiche. Wie in den Topographien zu erkennen, sind diese weiterhin bevorzugt an den Rändern der Körner lokalisiert.

Eine weitere Messserie, in der das Wachstum von LRS-Bereichen beobachtet werden kann, ist in Abb. 6.5 gezeigt. Aufgrund der langen Messdauer zur Aufnahme dieser Messserie ist sie mit Drift behaftet; daher ist der Bereich, der in allen Messungen zu sehen ist, durch ein grünes Rechteck markiert. Die Messspannung liegt in dieser Serie konstant bei $U_{\text {mess }}=0,8 \mathrm{~V}$. Diese Spannung ist niedriger als die in epitaktischen Manganatproben beobachtete kritische Schaltspannung von $U_{c}=3 \mathrm{~V},[60$ aber größer als in den Messungen in Abb. 6.1 und 6.4. Zu Beginn dieser Messserie (Abb. 6.5) sind innerhalb des gezeigten Probenbereichs wiederum einige wenige Stellen im LRS. Auch hier können diese LRSBereiche durch Schaltspannungen in vorherigen Messserien verursacht sein. Nach einer Zeit von 41 Minuten (in dieser Zeit wurde der Messbereich zehnmal gescannt) ist zu sehen, dass die Anzahl der LRS-Bereiche deutlich zugenommen hat. Dieser Trend setzt sich auch für die weiteren Messungen fort; dabei wurde nach 82 Minuten der Bereich 20 Mal seit der 

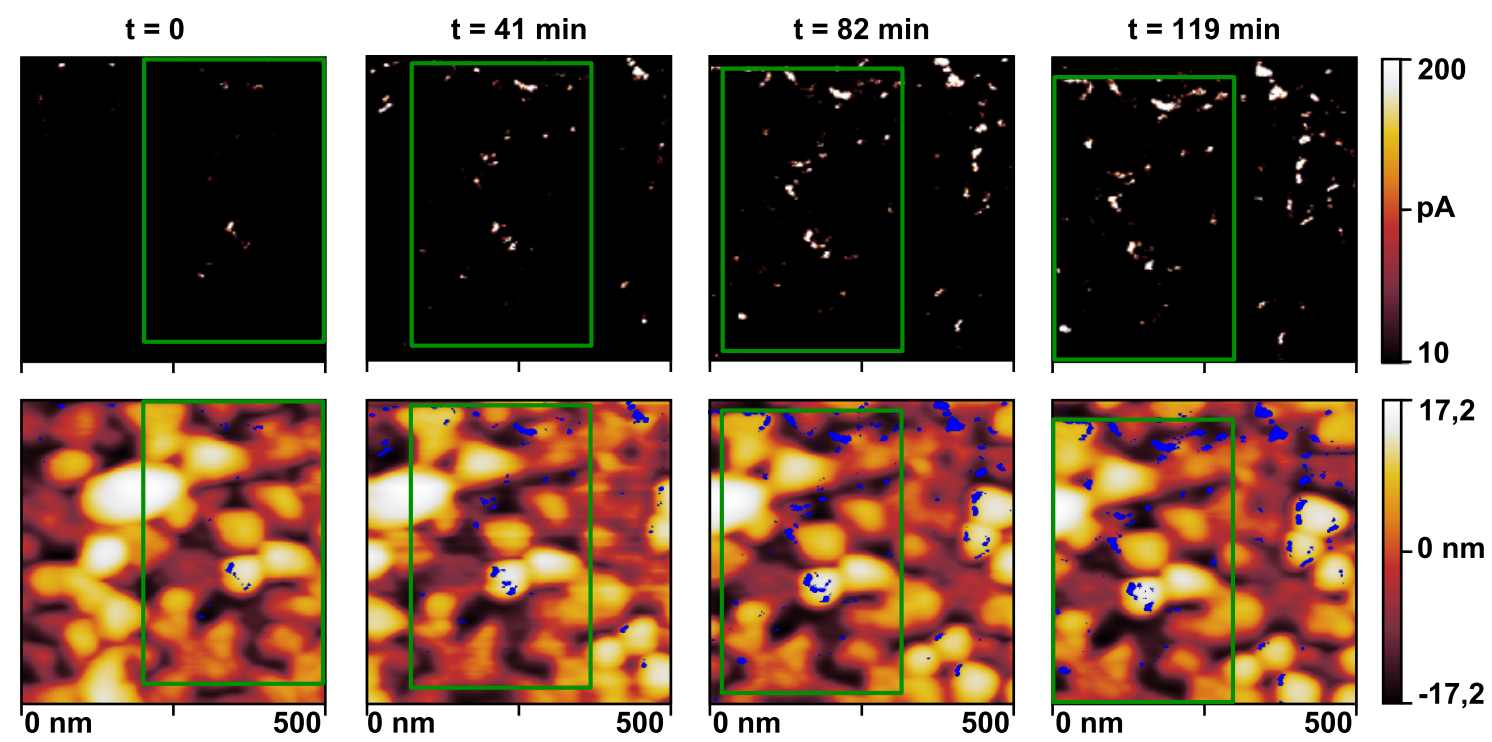

Abb. 6.5: C-AFM Messung: Der gezeigte Messbereich wurde wiederholt mit $U_{\text {mess }}=0,8 \mathrm{~V}$ aufgenommen. Aufgrund der Drift verändert sich der Messausschnitt, das grüne Rechteck markiert einen Probenbereich, der allen Messungen gemeinsam ist. In der oberen Reihe sind die Stromkarten gezeigt, in der unteren die jeweiligen Topographien. Im Laufe der Messungen vergrößert sich die Zahl und Fläche der LRS-Bereiche, insbesondere im Bereich der Korngrenzen.

ersten gezeigten Messung gescannt und nach 119 Minuten 29-fach. Mit einer Spannung von 0,8 V kann die Probe also sukzessive in den LRS geschaltet werden. Die untere Zeile von Abb. 6.5 zeigt die zu den Stromkarten zugehörigen Topographien. In diesen Abbildungen ist wiederum klar erkennbar, dass der LRS bevorzugt an den Rändern der Körner zu finden ist.

Ein gemeinsames Ergebnis der Messungen in Abb. 6.4 und 6.5 ist das Wachstum der LRSBereiche entlang von Korngrenzen. Durch wiederholtes Scannen mit $U_{\text {mess }}=0,8 \mathrm{~V}$ oder durch Spannungspulse mit $U_{\text {schalt }}=8 \mathrm{~V}$ entstehen immer mehr Bereiche mit einer erhöhten Leitfähigkeit, die sich entlang der Korngrenzen ausdehnen.

\subsubsection{Einfluss der Schichtdicke}

Im folgenden Abschnitt werden die Ergebnisse von C-AFM Messungen an einer $300 \mathrm{~nm}$ dicken LSMO $/ \mathrm{Al}_{2} \mathrm{O}_{3}$ Probe vorgestellt. Die veränderte Schichtdicke ist in der Topographie (siehe Abb. 6.6(a)) anhand der größeren Kornausdehnungen zu erkennen, wie in Abschnitt 5.4 beschrieben wird. Die laterale Ausdehnung der Körner beträgt etwa $90 \mathrm{~nm}$. Zusätzlich zeigt die Topographie einen erhöhten Anteil an Dreiecken, welches für viele (111)-orientierte Körner in diesem Probenbereich spricht.

In Abb. 6.6(b) ist die zur Topographie zugehörige Stromkarte gezeigt. Es ist bemerkenswert, dass - obwohl die erste Messung an der Schicht gezeigt ist - schon ein großer Anteil an LRS vorhanden ist. Die Spannung während dieser ersten Messung betrug lediglich $U_{\text {mess }}=0,1 \mathrm{~V}$. Dennoch fließt bei $15 \%$ der Messpunkte ein größerer Strom als $1 \mathrm{nA}$. Selbst für Ströme höher als $10 \mathrm{nA}$ sind es noch $8 \%$ der Messpunkte. Für eine Stromschwelle von $40 \mathrm{nA}$ finden sich noch $5 \%$ der Messpunkte, die oberhalb dieser Schwelle liegen. Dieser 


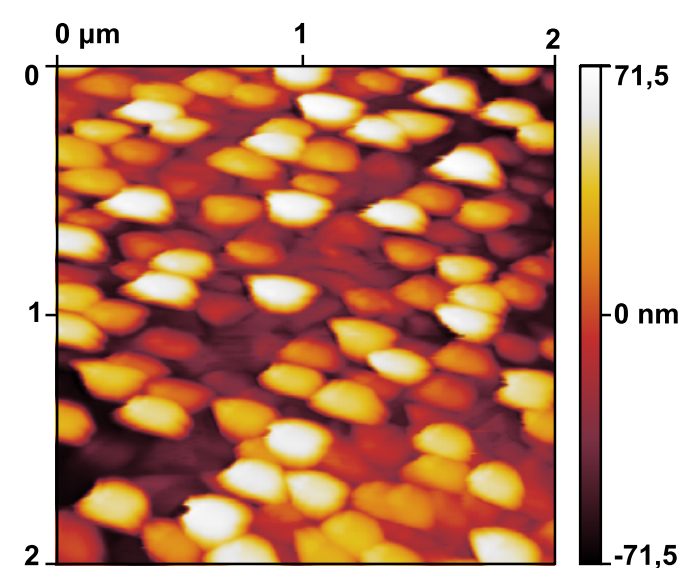

(a) Topographie

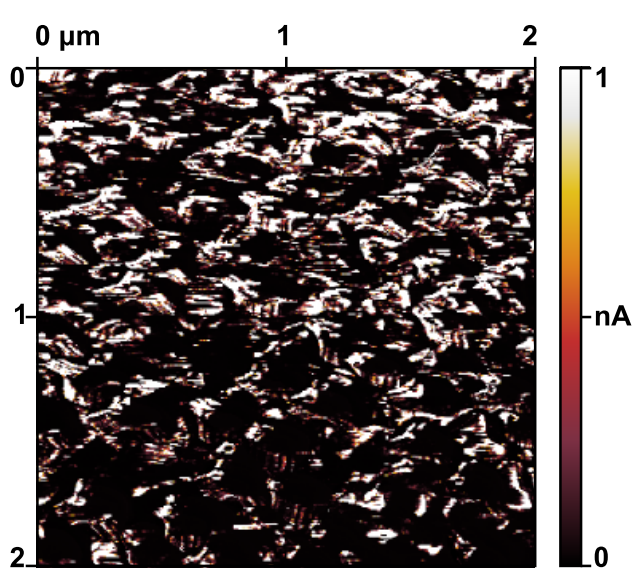

(b) Stromkarte

Abb. 6.6: Topographie und Stromkarte für eine $300 \mathrm{~nm}$ dicke LSMO-Schicht: Im Ausgangszustand (während der ersten Messung an dieser Probe) findet sich in der Stromkarte schon ein signifikanter Anteil an LRS $\left(U_{\text {mess }}=0,1 \mathrm{~V}\right)$.

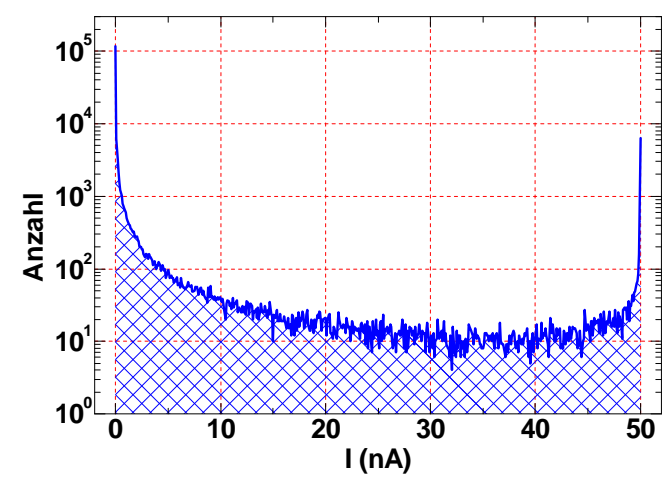

(a) Häufigkeitsverteilung der Stromkarte

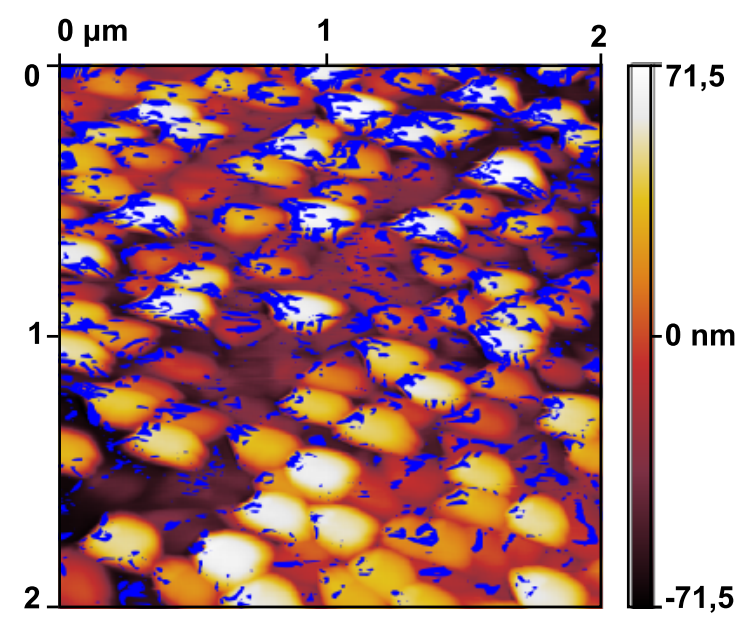

(b) Topographie (in Blau: Stromkarte)

Abb. 6.7: In (a) ist die Häufigkeitsverteilung der bei $U_{\text {mess }}=0,1 \mathrm{~V}$ aufgezeichneten Stromkarte aus Abb. 6.6(b) gezeigt. In (b) ist die mit der Stromkarte überlagerte Topographie der $300 \mathrm{~nm}$ dicken LSMO-Schicht im Ausgangszustand zu sehen.

Sachverhalt wird auch in der Häufigkeitsverteilung der Stromkarte (Abb. 6.7(a)) verdeutlicht. Ströme größer als $50 \mathrm{nA}$ werden in dem vorhanden Messaufbau durch den Vorverstärker abgeschnitten, daraus resultiert die große Zahl von Messpunkten bei $50 \mathrm{nA}$.

Um die Verteilung der LRS-Bereiche im Bezug auf die Morphologie zu untersuchen, ist in Abb. 6.7(b) die Topographie mit der Stromkarte überlagert. Hierfür werden alle Bereiche, die einen größeren Stromfluss als $1 \mathrm{nA}$ zeigen, als LRS in Blau eingezeichnet. Es zeigt sich, dass auch für die $300 \mathrm{~nm}$ dicke LSMO-Schicht die LRS-Bereiche bevorzugt an den Korngrenzen zu finden sind. Bei einer quantitativen Analyse der Übereinstimmung der Gradientenkarte der Topographie und der Stromkarte ergeben sich geringere Werte als bei der $44 \mathrm{~nm}$ dicken Schicht: Bei einer Schwellwertmarkierung von $S=25 \%$ und einer Zuordnung zum LRS für Bereiche mit Strömen von $I>40 \mathrm{nA}\left(U_{\text {mess }}=0,1 \mathrm{~V}\right)$ liegt die Übereinstimmung bei $49 \%$. Wird $S=41 \%$ gewählt, so sind es $68 \%(I>40 \mathrm{nA})$. 


\subsection{Schaltexperimente an Mikrostrukturen}

Während in Abschnitt 6.1 das elektrische Widerstandsschalten mittels C-AFM Messungen untersucht wird, folgt nun eine Charakterisierung der Schalteffekte in strukturierten Proben. Die Mikrostrukturen werden mittels Elektronenstrahl-Lithographie präpariert, wie in Abschnitt 4.4 beschrieben wird. Erste Messungen des elektrisch induzierten Widerstandsschaltens in mikrostrukturierten, nanokolumnaren Manganatschichten wurden im Rahmen der Doktorarbeit von Esseling durchgeführt. [88]

Bei den Widerstandsmessungen an den Mikrostrukturen trägt eine Vielzahl von Manganatsäulen zum elektronischen Transportverhalten bei. Die Strukturgrößen sind typischerweise im Bereich von wenigen Mikrometern, während die Korngrößen der einzelnen Manganatsäulen bei $30 \ldots 90 \mathrm{~nm}$ liegen. Obwohl dieses Größenverhältnis nur die Untersuchung des gemittelten Verhaltens vieler Manganatsäulen zulässt, werden dennoch starke Unterschiede in den Ergebnissen der Schaltexperimente an verschiedenen Mikrostrukturen beobachtet.

Die nachfolgenden Experimente wurden an Manganatproben mit Schichtdicken zwischen $40 \ldots 60 \mathrm{~nm}$ durchgeführt, die auf (0001)-orientierten $\mathrm{Al}_{2} \mathrm{O}_{3}$-Substraten deponiert wurden. Messungen an Schichten auf anderen Substratorientierungen sind gekennzeichnet.

\subsubsection{Typische Schaltcharakteristiken der mikrosktrukturierten Proben}

In Abb. 6.8(a) ist das Messschema der Widerstandsschaltkennlinien, wie sie in den Experimenten an den Mikrostrukturen durchgeführt werden, skizziert. Zur Messung der Schaltcharakteristiken werden Spannungspulse (Pulsdauer $t=1 \mathrm{~s}$ ) mit variierender Amplitude angelegt. Der Stromfluss wird während dieser Spannungspulse gemessen. Dabei wird jeweils ein Puls mit unveränderter Amplitude (Messpuls, $U_{\text {mess }}$ ) mit einem Puls variabler Größe (Schaltpuls, $U_{\text {schalt }}$ ) abgewechselt. Der Schaltpuls wird von $U_{\text {schalt }}=0$ bis zu einer maximalen, positiven Spannung von $+U_{\max }$ geregelt, danach wird die Schaltspannung von $+U_{\max }$ bis $-U_{\max }$ und schließlich wieder auf $U_{\text {schalt }}=0$ gefahren. In Analogie zu magnetischen Hysteresekurven wird im Folgenden ein solcher Messablauf als Hysterese bezeichnet. Eine vollständige Hysteresekurve bedeutet entsprechend, dass $U_{\text {schalt }}$ von $0 \rightarrow+U_{\max } \rightarrow-U_{\max } \rightarrow 0$ verändert wird. Hierbei wird zwischen den einzelnen Schaltpulsen immer wieder ein Messpuls angelegt. Dieses erlaubt eine gleichzeitige Untersuchung des Widerstands in variierenden, elektrischen Feldern bzw. Stromdichten $\left(R_{\text {schalt }}=U_{\text {schalt }} / I_{\text {schalt }}\right)$ und bei einer konstanten Spannung $\left(R_{\text {mess }}=U_{\text {mess }} / I_{\text {mess }}\right)$ in Abhängigkeit von der elektrischen Vorgeschichte.

In Abb. 6.8 findet sich eine typische Widerstandsschaltmessung, die an einer mikrostrukturierten, nanokolumnaren LSMO-Schicht aufgenommen wurde. Die Mikrostruktur hat einen Elektrodenabstand von $a=1 \mu \mathrm{m}$ und eine Stegbreite von $b=6,5 \mu \mathrm{m}$. Zur Verdeutlichung der Bezeichnungen $a$ und $b$ ist in Abb. 6.8(a) eine beispielhafte REM-Aufnahme von einer Mikrostruktur gezeigt. In Abb. 6.8(b) ist der Schaltstrom als Funktion der Schaltspannung aufgetragen. Die zugehörigen Widerstände $R_{\text {mess }}$ und $R_{\text {schalt }}$ sind in Abb. 6.8(c) und (d) ebenfalls als Funktion von $U_{\text {schalt }}$ abgebildet.

Am Anfang der Hysteresemessung in Abb. 6.8 ist die Probe in einem tiefohmigen Zustand (Pfeil 1). Die Schaltspannung wird schrittweise erhöht, wodurch sich der Widerstand $\left(R_{\text {mess }}\right.$ und $\left.R_{\text {schalt }}\right)$ ab einer kritischen Spannung von $U_{\mathrm{c} \text {, schalt }}=+2 \mathrm{~V}$ vergrößert. Nach einigen Schaltpulsen ist ein hochohmiger Widerstandszustand (HRS) eingestellt. $R_{\text {mess }}$ verändert sich mit zunehmender Schaltspannung nicht weiter (Pfeil 2). Im nächsten Schritt der Hysteresekurve wird die Schaltspannung von $+U_{\max } \rightarrow-U_{\max }$ reduziert. Dabei verändert 


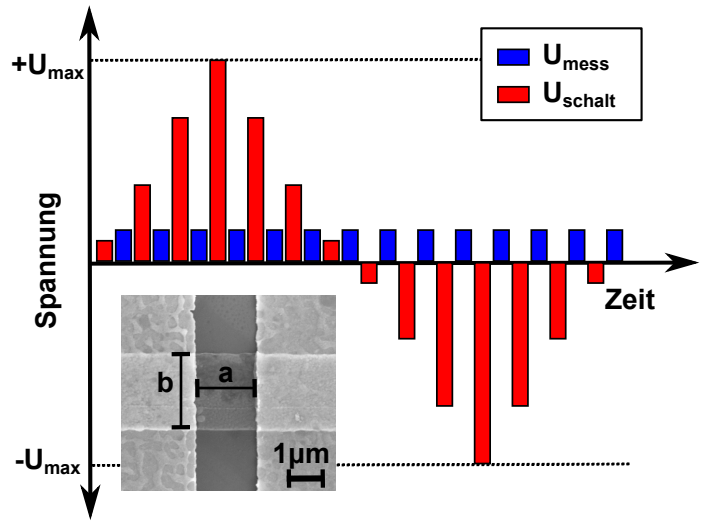

(a) schematische Messabfolge

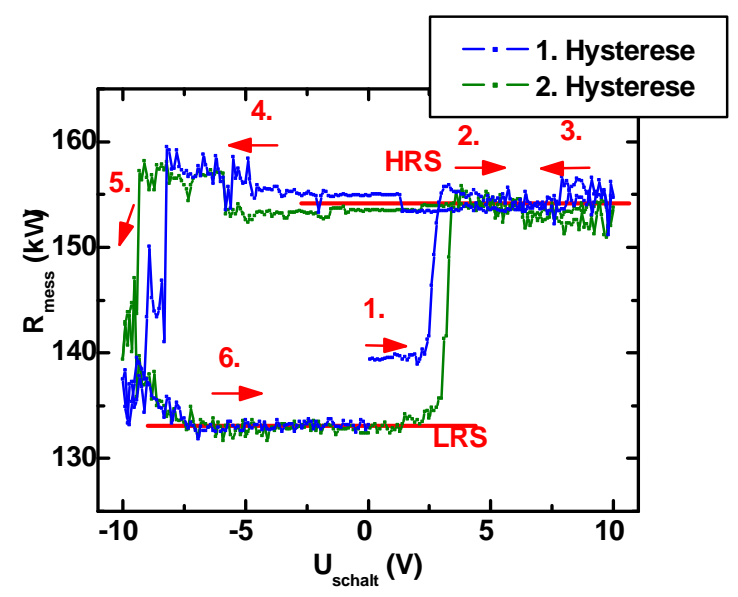

(c) $R_{\text {mess }}\left(U_{\text {schalt }}\right)$ mit $U_{\text {mess }}=1 \mathrm{~V}$

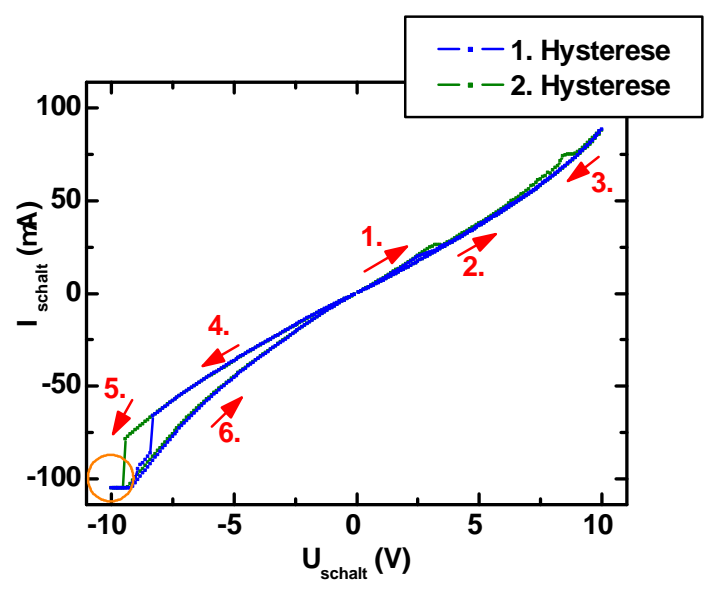

(b) $I_{\text {schalt }}\left(U_{\text {schalt }}\right)$

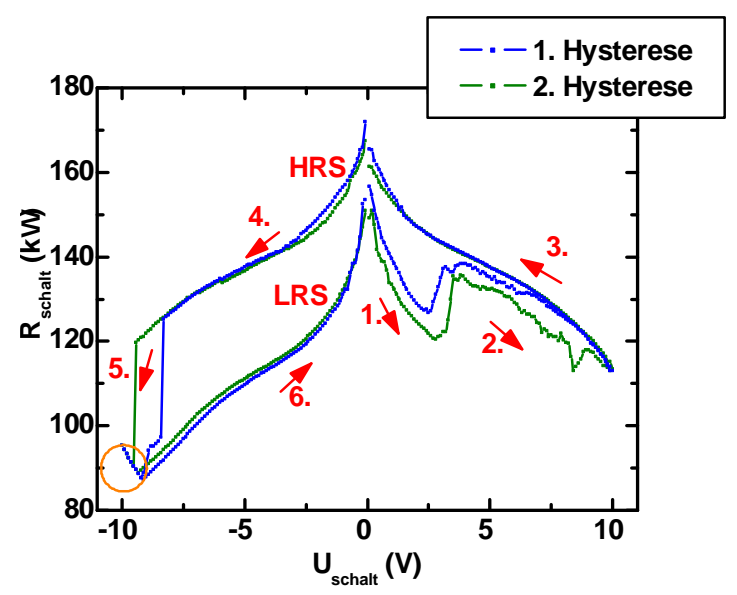

(d) $R_{\text {schalt }}\left(U_{\text {schalt }}\right)$

Abb. 6.8: Schalt-Kennlinien einer nanokolumnaren LSMO-Mikrostruktur ( $T=100 \mathrm{~K}$, Elektrodenabstand $a=1 \mu \mathrm{m}$, Stegbreite $b=6,5 \mu \mathrm{m}$ (für die Bedeutung von $a$ und $b$ siehe die schematische REM-Aufnahme in (a)), $\mathrm{Al}_{2} \mathrm{O}_{3}$ (012)-orientiert). Die rote Nummerierung gibt die Messreihenfolge an. Bereiche, die mit einem orangen Kreis gekennzeichnet sind, wurden aufgrund eines Strom-compliance nicht aufgenommen.

sich der Messwiderstand bis zu einer kritischen Schaltspannung von $U_{\mathrm{c} \text {, schalt }}=-8 \mathrm{~V}$ nur geringfügig (Pfeil 3, 4). Ab $U_{\mathrm{c} \text {, schalt }}=-8 \mathrm{~V}$ (Pfeil 5) schaltet der Widerstand $\left(R_{\text {mess }}\right.$ und $\left.R_{\text {schalt }}\right)$ wieder in einen tiefohmigen Zustand (LRS). Im LRS hat die Struktur einen kleineren Widerstand als zu Beginn der Messung.

Im zweiten Durchlauf der Hysterese schaltet der Widerstand wiederum bei einer positiven Schaltspannung von $U_{\mathrm{c} \text {, schalt }} \approx+2 \mathrm{~V}$ in den HRS. Bei einer negativen Schaltspannung von $U_{\mathrm{c}, \text { schalt }} \approx-8 \mathrm{~V}$ wird der LRS eingestellt. Der am Anfang der 1. Hysterese vorliegende, tiefohmige Widerstandswert (Pfeil 1) ist auf die Vorgeschichte der Probe zurückzuführen, wie in der in Abschnitt 6.2.2 vorgestellten Messserie demonstriert wird.

In den Mikrostrukturmessungen werden oft mehr als nur zwei remanente Widerstandswerte beobachtet, wie auch in der Messung in Abb. $6.8 \mathrm{zu}$ sehen ist. Hierbei befindet sich 


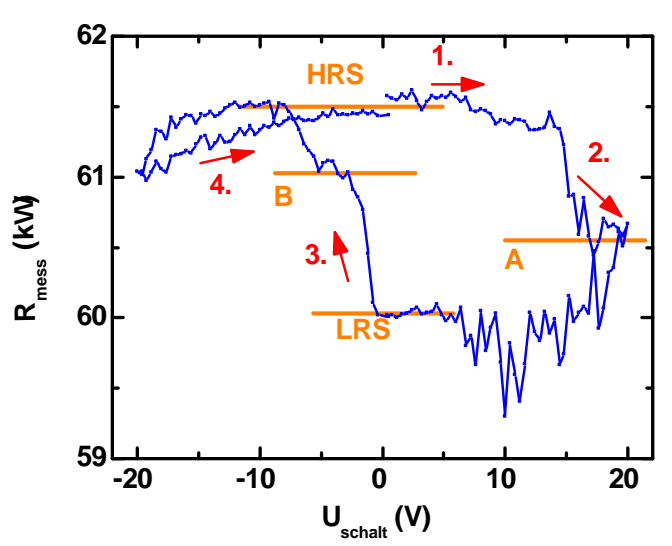

(a) 1. Hysterese

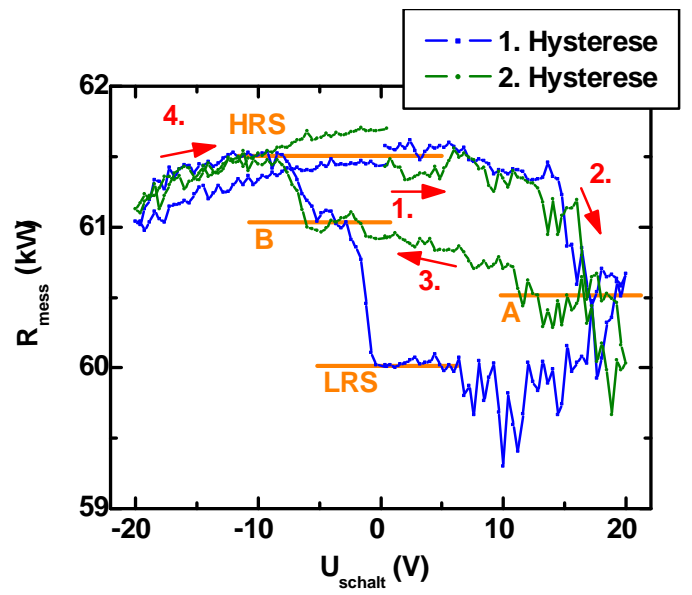

(b) 1. und 2. Hysterese

Abb. 6.9: Widerstandsstufen zwischen HRS und LRS $\left(T=10 \mathrm{~K}, U_{\text {mess }}=0,5 \mathrm{~V}\right)$. In diesen Messungen wurden die Al-Bonds ohne Mikrostrukturierung direkt auf der Probenoberfläche angebracht.

die Struktur zu Beginn in einem Widerstandszustand, der zwischen dem HRS und LRS liegt. In diesem Zusammenhang wird der HRS als der größte, der LRS als der kleinste Widerstandszustand definiert. In Abb. 6.9 ist ein weiteres Beispiel für die Messung einer Mikrostruktur gezeigt, in der mehrere Widerstandszustände beobachtet werden. Am Anfang der ersten Hysterese ist die Struktur im HRS (Abb. 6.9(a), Pfeil 1). Ab einer kritischen Schaltspannung von $U_{\mathrm{c} \text {, schalt }} \approx+14 \mathrm{~V}$ verändert sich der Widerstand in den Zustand A (orange Linie, Pfeil 2). Während die Schaltspannung von $+U_{\max } \rightarrow 0$ reduziert wird, verringert sich der Widerstand in den LRS. Ab einer Spannung von $U_{\mathrm{c} \text {, schalt }}=-0,4 \mathrm{~V}$ schaltet der Widerstand wieder in den HRS, wobei auch hier eine Zwischenstufe (B) zwischen HRS und LRS beobachtet wird (Pfeil 3). Beim zweiten Spannungs-Durchlauf, siehe Abb. 6.9(b), schaltet der Widerstand auf dem Weg von $0 \rightarrow U_{\max }$ ab $U_{\mathrm{c} \text {, schalt }}=+16 \mathrm{~V}$ zunächst wieder in den Widerstandswert A und dann $\left(a b U_{\mathrm{c} \text {, schalt }}=+19,2 \mathrm{~V}\right)$ in den LRS. Auf dem Rückweg $\left(U_{\max } \rightarrow-U_{\max }\right)$ verändert sich der Widerstand über die Stufen A und B wieder in den HRS (bei negativer Schaltspannung). Auch weitere Messungen dieser Struktur, die bei Temperaturen zwischen $10 \ldots 100 \mathrm{~K}$ aufgenommen wurden, zeigen immer wieder die Widerstandsstufen A, B, HRS und LRS.

Insgesamt zeigen die meisten Schaltexperimente, dass die Widerstandswerte im HRS wesentlich weniger als im LRS streuen; entsprechend kann der HRS stabiler als der LRS eingestellt werden. Ein Extremfall hiervon ist in Abb. 6.10(a) demonstriert. In dieser Messung wurden vielfach wiederholte Hystersen $\left(0 \rightarrow+U_{\max }=+20 \mathrm{~V} \rightarrow-U_{\max }=-20 \mathrm{~V} \rightarrow 0\right)$ an derselben Struktur - ohne Veränderung sonstiger Messparameter wie beispielsweise der Temperatur - aufgenommen. Dabei fällt auf, dass der LRS in den unterschiedlichen Messzyklen stark verschieden sein kann, der HRS-Wert dagegen kaum variiert. Die Messung zeigt, dass der LRS in dieser Struktur nicht reproduzierbar eingestellt werden kann. Die Reproduzierbarkeit von Schaltcharakteristiken ist für unterschiedliche Mikrostrukturen sehr verschieden: Während in Abb. 6.10(a) fast alle Durchläufe (mit gleicher, maximaler Schaltspannung) unterschiedliche tiefohmige Zustände zeigen, ist dieses beispielsweise für die in Abb. 6.8 gezeigte Messung (an einer anderen Mikrostruktur) nicht der Fall. 


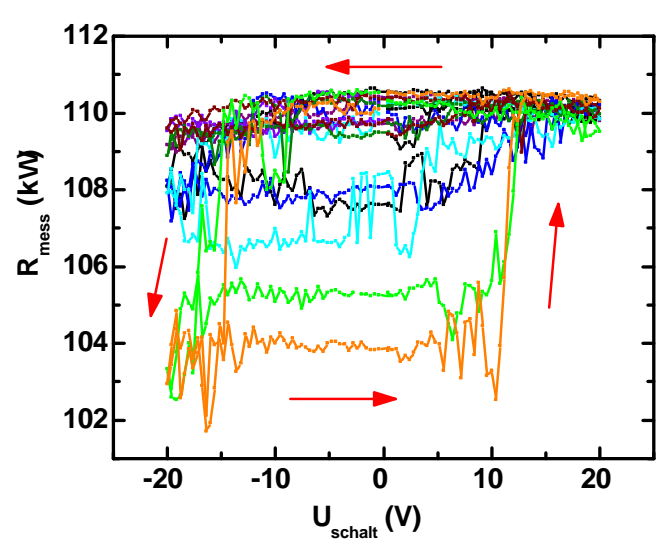

(a) Struktur I

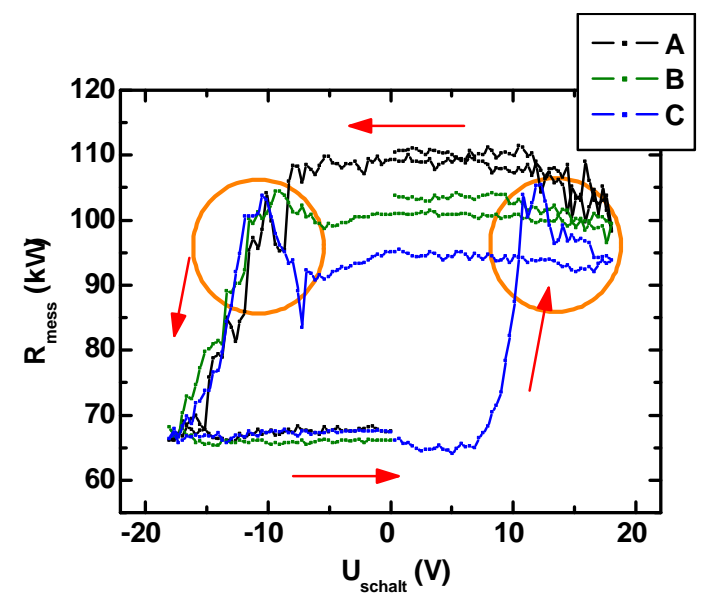

(b) Struktur II

Abb. 6.10: Reproduzierbarkeit der Mikrostruktur-Kennlinien: In den gezeigten Messungen wurden wiederholt Hysteresen mit unveränderter maximaler Schaltspannung aufgenommen, wobei in den Strukturen I (a) und II (b) deutliche Unterschiede in den einzelnen Messdurchläufen beobachtet werden $(T=10 \mathrm{~K}$, $U_{\text {mess }}=0,5 \mathrm{~V}$ ). Bei den Messungen der Struktur II (b) wurden zwischen der Hysterese A und B weitere, hier nicht gezeigte Messungen durchgeführt.

Weiterhin wird beobachtet, dass manche Schaltprozesse erst im Laufe von wiederholten Messzyklen auftreten. Ein Beispiel hierfür ist in Abb. 6.10(b) gezeigt. Auch in dieser Struktur wurde wiederholt die gleiche Hysterese $\left(0 \rightarrow+U_{\max }=+20 \mathrm{~V} \rightarrow-U_{\max }=-20 \mathrm{~V} \rightarrow 0\right)$ gemessen. Erst nach einigen Durchläufen wird ein weiterer Schaltprozess beobachtet, dieser ist in der Abb. 6.10(b) mit orangen Kreisen gekennzeichnet.

\section{Kritische Schaltparameter}

In den Abbildungen 6.8 bis 6.10 werden typische Schaltcharakteristiken für eine Reihe von Mikrostrukturen präsentiert. An dieser Stelle werden die Gemeinsamkeiten aller Mikrostrukturmessungen zur besseren Übersicht in der folgenden Liste zusammengefasst.

\section{- EPIR-Betrag}

Die Größe der Widerstandsänderung zwischen HRS und LRS wird für die strukturierten Proben ebenfalls nach Gleichung 6.1 berechnet. Für die Mikrostruktur in Abb. 6.8 ergibt sich

$$
\mathrm{EPIR}=\left(\frac{R_{\text {mess, HRS }}-R_{\text {mess, LRS }}}{R_{\text {mess, } \text { LRS }}}\right) \cdot 100 \%=16 \% .
$$

Insgesamt variiert dieser Wert sehr stark für unterschiedliche Strukturen und Proben. Es werden EPIR-Beträge zwischen $=5 \ldots 240 \%$ beobachtet. Hierbei ist zu berücksichtigen, dass durch den unterschiedlichen Verlauf der I(U)-Kennlinien in HRS und LRS der EPIR eine Funktion der Spannung ist. Im Vergleich zu den Mikrostrukturmessungen findet sich in den C-AFM Messungen EPIR C-AFM $_{2} \approx 26000 \%$.

- Verhältnis der kritischen Schaltspannungen $\left|\frac{U_{\mathbf{c}, \text { HRS } \rightarrow \text { LRS }}}{U_{\mathbf{c}, \text { LRS } \rightarrow \text { HRS }}}\right|$

Die kritische Schaltspannung $U_{\mathrm{c}, \text { HRS } \rightarrow \text { LRS }}$, die den Widerstand von HRS nach LRS 


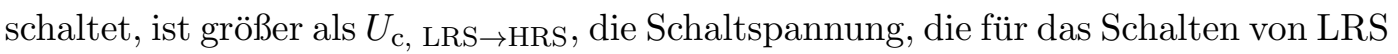
nach HRS verantwortlich ist. Für das Verhältnis der kritischen Schaltspannungen wird

$$
U_{\mathrm{c}}^{*}=\left|\frac{U_{\mathrm{c}, \mathrm{HRS} \rightarrow \mathrm{LRS}}}{U_{\mathrm{c}, \mathrm{LRS} \rightarrow \mathrm{HRS}}}\right|=1,1 \ldots 5,5
$$

ermittelt, wobei der Mittelwert $\overline{U_{\mathrm{c}}^{*}}=2,4 \pm 1,5$ ist. Allerdings ist die Bestimmung der kritischen Schaltspannungen nicht in allen Fällen eindeutig, da oft Zwischenstufen zwischen HRS und LRS beobachtet werden (siehe zum Beispiel Abb. 6.9). In diesen Fällen wurde die Spannung, bei welcher der größte Schaltprozess (EPIR-Betrag) initiiert wird, für $U_{\mathrm{c}}$ verwendet.

\section{- Stromdichten und elektrische Feldstärken}

Die Strukturgrößen der verwendeten Proben wurden stark variiert. Es wurden Mikrostrukturen mit Stegbreiten zwischen $b=1 \ldots 145 \mu \mathrm{m}$ und Elektrodenabständen zwischen $a=0,8 \ldots 33 \mu \mathrm{m}$ präpariert. In allen diesen Strukturen wird bipolares Widerstandsschalten beobachtet. Es zeigt sich sogar, dass auch ohne Mikrostrukturierung bipolares Schalten beobachtet werden kann. Ein Beispiel hierfür ist in Abb. 6.9 gezeigt, in dieser Messung wurden die Bonds direkt auf der Probe befestigt.

Die Berechnung der Stromdichte und der elektrischen Feldstärke ist in den nanokolumnaren Manganatschichten problematisch, da diese Schichten eine sehr inhomogene Widerstandsverteilung zeigen. Dadurch sind lokal sehr unterschiedliche elektrische Feldstärken und Stromdichten zu erwarten. Wird trotz dieser Problematik die Stromdichte $J$ aus den Abmessungen der Mikrostrukturen bestimmt, ergibt sich

$$
J=\frac{I}{d \cdot b}=10^{7} \ldots 10^{9} \mathrm{~A} / \mathrm{m}^{2} .
$$

Dabei entspricht $d$ der Schichtdicke. In der gleichen Weise kann auch die elektrische Feldstärke $E$ berechnet werden. Diese ist gegeben durch

$$
E=\frac{U}{a}=10^{5} \ldots 10^{7} \mathrm{~V} / \mathrm{m}
$$

\section{- Verwendete Elektrodenmaterialien}

Als Elektrodenmaterial wird in den mittels Elektronenstrahl-Lithographie präparierten Strukturen zunächst eine $5 \mathrm{~nm}$ dicke Chromschicht, gefolgt von einer $50 \ldots 100 \mathrm{~nm}$ dicken Goldschicht, verwendet. Widerstandsschalteffekte werden auch beobachtet, wenn Aluminium-Bonddrähte direkt auf der Probe befestigt werden. In den C-AFM Messungen ist die mobile Elektrode ein mit Platin beschichteter Cantilever aus Silizium. Für alle diese Elektrodenmaterialien wird bipolares Widerstandsschalten beobachtet.

\section{- Einfluss der Orientierungen der Manganatkörner}

Durch die Verwendung von unterschiedlich orientierten Saphir-Substraten wird das Verhältnis der Orientierungen der Manganatkörner zueinander modifiziert. Die Unterschiede in den Schaltparametern der Mikrostrukturen auf einer Probe sind allerdings genauso groß, wie zwischen den Strukturen auf unterschiedlich orientierten Saphir-Substraten. Es kann also keine Abhängigkeit der Schaltcharakteristiken von der Orientierung des Substrates beobachtet werden.

Diese Auflistung gibt eine Übersicht über die kritischen Schaltparameter der Mikrostrukturmessungen. Im Folgenden wird eine Messserie mit einer Variation der maximalen Schaltspannung $U_{\max }$ in sukzessiven Messungen der gleichen Struktur und der Einfluss eines externen Magnetfeldes und der Temperatur vorgestellt. 


\subsubsection{Variation der maximalen Schaltspannung $U_{\max }$}

Eine Variation der maximalen Schaltspannung $U_{\max }$ in aufeinanderfolgenden Hysteresen ist in Abb. 6.11 gezeigt. Liegt $U_{\max }$ im Bereich der kritischen Schaltspannungen, kann die Stabilität der Widerstandszustände zwischen HRS und LRS untersucht werden.

Die erste Hysterese $\left(U_{\text {schalt }}: 0 \rightarrow+U_{\max } \rightarrow-U_{\max } \rightarrow 0\right)$ dieser Messserie ist in Abb. 6.11(a) und (b) zu finden, in dieser wird $U_{\max }= \pm 20 \mathrm{~V}$ gewählt. Am Anfang der Messung ist die Probe in einem Widerstandszustand, der etwas kleiner als der HRS ist und aus vorherigen Messungen resultiert. Ab einer Spannung von $U_{\mathrm{c} \text {, schalt }}=+10 \mathrm{~V}$ wird der Widerstand der Struktur in den LRS geschaltet. Der Schaltprozess aus dem HRS in den LRS erstreckt sich dabei über zahlreiche, immer größer werdende Schaltpulse. Der LRS ist ab einer Schaltspannung von $U_{\text {schalt }}=+14 \mathrm{~V}$ stabil. Die Struktur bleibt in diesem Zustand, bis die Schaltspannung einen kritischen Betrag von $U_{c, \text { schalt }}=-7,2 \mathrm{~V}$ erreicht. Ab dieser Spannung wird der Widerstand der Probe wieder größer, bis er schließlich im HRS ankommt. Im weiteren Verlauf der Hysterese $\left(U_{\text {schalt }}\right.$ wird von $-12,5 \mathrm{~V} \rightarrow-20 \mathrm{~V}$ variiert) reduziert sich der Widerstand auf den Wert A, der zwischen HRS und LRS liegt.

In der zweiten Messung (siehe Abb. 6.11(c) ) beträgt die maximale Schaltspannung $U_{\max }=$ \pm 11 V. Dieses entspricht in etwa der kritischen Schaltspannung, ab der im ersten Messzyklus (Abb. 6.11(b) der Widerstand aus dem HRS in den LRS schaltet. Der zweite Messzyklus startet im Widerstandszustand A. Während $U_{\text {schalt }}$ von $0 \rightarrow+U_{\max }=+11 \mathrm{~V}$ vergrößert wird, beginnt der Widerstand ab $U_{\text {schalt }}=+6 \mathrm{~V}$ in den HRS zu schalten. Auch hier sind mehrere Schaltpulse notwendig, um den HRS einzustellen. Für Spannungen zwischen $U_{\max }= \pm 11 \mathrm{~V}$ verändert sich der Widerstand nicht weiter und bleibt im HRS.

In der nächsten Messung (Abb. 6.11(d)) wird $U_{\max }= \pm 17 \mathrm{~V}$ gewählt. Der Widerstand schaltet auf dem Weg von $0 \rightarrow+U_{\max }$ aus dem HRS in einen Zustand zwischen A und LRS. Dieser Widerstandszustand ist stabil, solange $U_{\text {schalt }}$ zwischen $+U_{\max }$ und $U_{\text {schalt }}=-6 \mathrm{~V}$ bleibt. Ab dieser Spannung schaltet der Widerstand wie in den vorherigen Messzyklen über den HRS in einen tiefohmigeren Wert. Allerdings wird zwischen $-U_{\max }=-17 \mathrm{~V}$ und $U_{\text {schalt }}=0$ ein kleinerer Widerstand als A eingestellt.

In der vierten Messung (Abb. 6.11(e) $)$ wird schließlich wieder eine maximale Schaltspannung von $U_{\max }= \pm 20 \mathrm{~V}$ verwendet. Für $U_{\text {schalt }}: 0 \rightarrow+U_{\max }$ wird wie zuvor über den HRS ein tiefohmiger Widerstandszustand erreicht. Dieser liegt allerdings etwas über dem urspünglichen LRS-Wert. Auf dem Abschnitt von $+U_{\max } \rightarrow-U_{\max }$ schaltet der Widerstand wieder über den HRS in den Zustand A.

Die fünfte Messung (Abb. 6.11(f) $)$ ist ähnlich zu der vorherigen. In diesen beiden Messungen wird $U_{\max }= \pm 20 \mathrm{~V}$ gewählt. Am Anfang der fünften Messung $\left(U_{\text {schalt }}: 0 \rightarrow+U_{\max }\right)$ schaltet der Widerstand von A über den HRS in den gleichen (modifizierten) LRS wie in der vierten Messung. Aus diesem verändert sich der Widerstand wiederum auf dem Weg von $+U_{\max } \rightarrow-U_{\max }$ über den HRS in den gleichen Widerstandszustand wie am Ende der Messung drei (in der allerdings $U_{\max }= \pm 17 \mathrm{~V}$ beträgt).

Ein wichtiger Aspekt dieser Messungen ist, dass die Zwischenzustände zwischen den Widerständen HRS und LRS remanent sind. Diese können systematisch durch die entsprechenden Schaltspannungen eingestellt werden. 


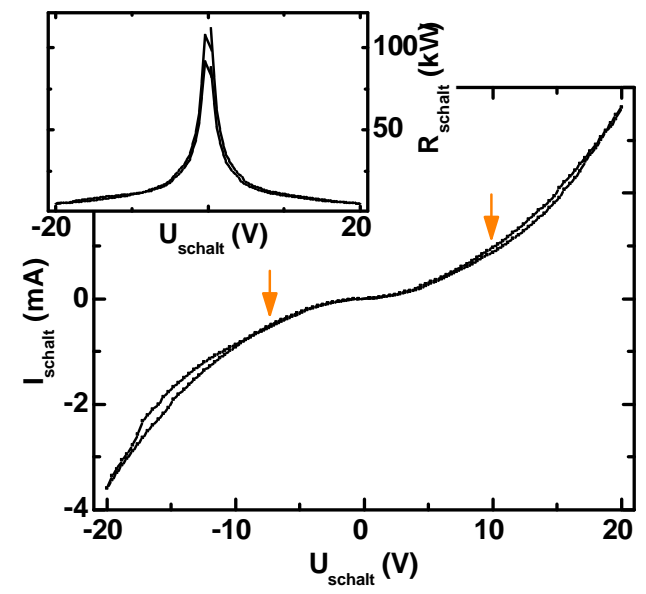

(a) 1. Hysterese, $U_{\max }= \pm 20 \mathrm{~V}$

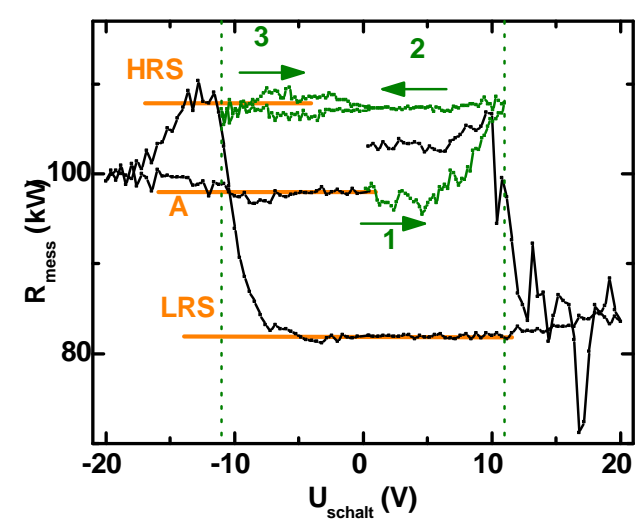

(c) 2. Hysterese, $U_{\max }= \pm 11 \mathrm{~V}$

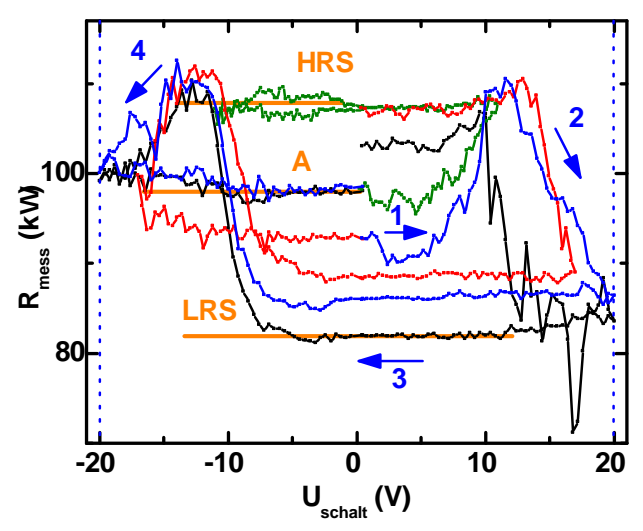

(e) 4. Hysterese, $U_{\max }= \pm 20 \mathrm{~V}$

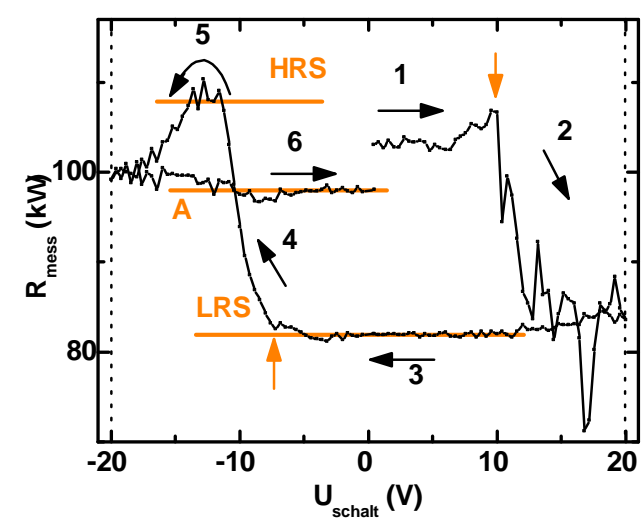

(b) 1 . Hysterese, $U_{\max }= \pm 20 \mathrm{~V}$

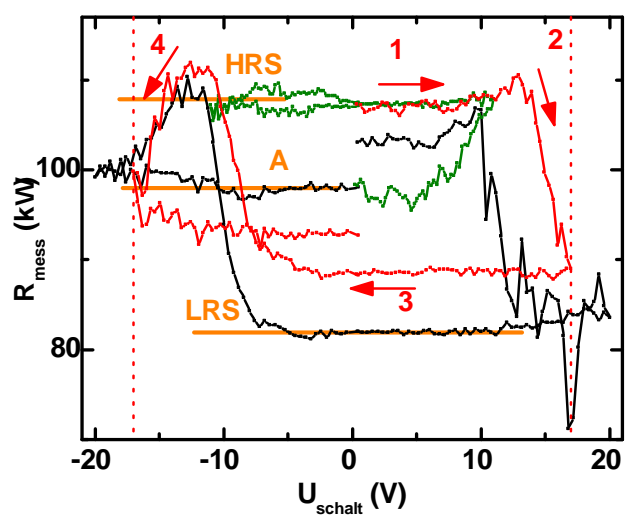

(d) 3. Hysterese, $U_{\max }= \pm 17 \mathrm{~V}$

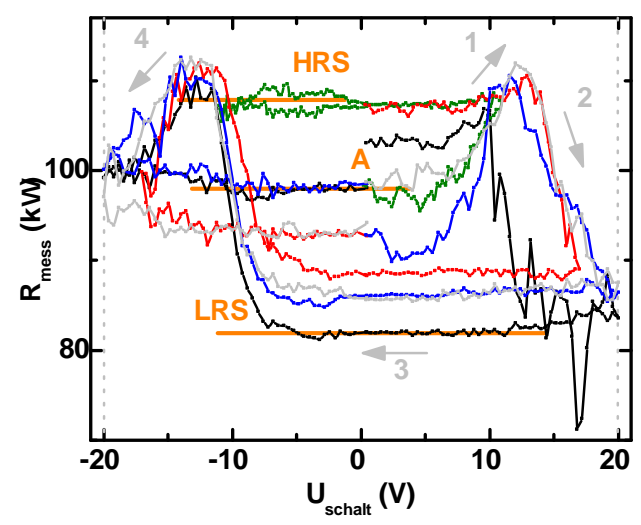

(f) 5 . Hysterese, $U_{\max }= \pm 20 \mathrm{~V}$

Abb. 6.11: Eine Variation der maximalen Schaltspannung $U_{\max }$ zeigt remanente Widerstandszustände zwischen HRS und LRS $\left(T=95 \mathrm{~K}, U_{\text {mess }}=0,5 \mathrm{~V}\right.$, Elektrodenabstand $a=20 \mu \mathrm{m}$, Stegbreite $b=135 \mu \mathrm{m})$. 


\subsubsection{Widerstandsschalten und Magnetowiderstand}

In Kap. 5.3 wird das magnetoresistive Verhalten der nanokolumnaren Manganatschichten gezeigt, in denen ein Kleinfeld-Magnetowiderstand (LFMR) beobachtet wird. Die wechselseitige Kombination dieses LFMR und des elektrischen Widerstandsschaltens wird in diesem Abschnitt untersucht. Dabei werden $R(H)$-Kurven im HRS und LRS gemessen, wodurch sich insgesamt vier verschiedene Widerstandswerte ergeben. Die systematische Variationsgröße der nachfolgenden Messungen ist der Schaltstrom (in den vorherigen Messungen wurde die Schaltspannung systematisch variiert).

In Abb. 6.12(a) ist der Schaltwiderstand als Funktion des Schaltstroms aufgetragen. Die Messung dieser Kurve wurde im HRS und im LRS unterbrochen, um $R(H)$-Kurven aufzunehmen. Diese $R(H)$-Kuven sind in Abb. 6.12(b) abgebildet. Sowohl im HRS als auch im LRS wird die, auch an den unstrukturierten Proben beobachtete, magnetoresistive Abhängigkeit gefunden (Kap. 5.3). Es ergeben sich jeweils zwei verschiedene Widerstandswerte beim Koerzitivfeld in Abhängigkeit von der magnetischen Vorgeschichte. Durch die wechselseitige Kombination des elektrischen und magnetischen Feldes können daher insgesamt vier remanente Widerstandswerte eingestellt werden $\left(R_{\mathrm{HRS}}\right.$, HMS,$R_{\mathrm{HRS}}$, LMS,$R_{\mathrm{LRS}}$, HMS und $R_{\text {LRS, LMS }}$ ). Die $R(H)$-Kurven im HRS und LRS unterscheiden sich im Rahmen der Messgenauigkeit lediglich um einen konstanten Widerstandsbetrag. Für den Magnetowiderstand $\operatorname{LFMR}\left(H=H_{C}\right)=\left(R_{\mathrm{HMS}}-R_{\mathrm{LMS}}\right) / R_{\mathrm{LMS}}$ ergibt sich im hochohmigen Zustand $\mathrm{LFMR}_{\mathrm{HRS}}=8 \%$ und im tiefohmigen Zustand $\mathrm{LFMR}_{\mathrm{LRS}}=9,5 \%$. Für das elektrische Widerstandsschalten in Abb. 6.12(a) wird EPIR $=\left(R_{\mathrm{HRS}}-R_{\mathrm{LRS}}\right) / R_{\mathrm{LRS}}=15,6 \%$ berechnet $(I=10 \mu \mathrm{A}, H=0 \mathrm{~T})$.

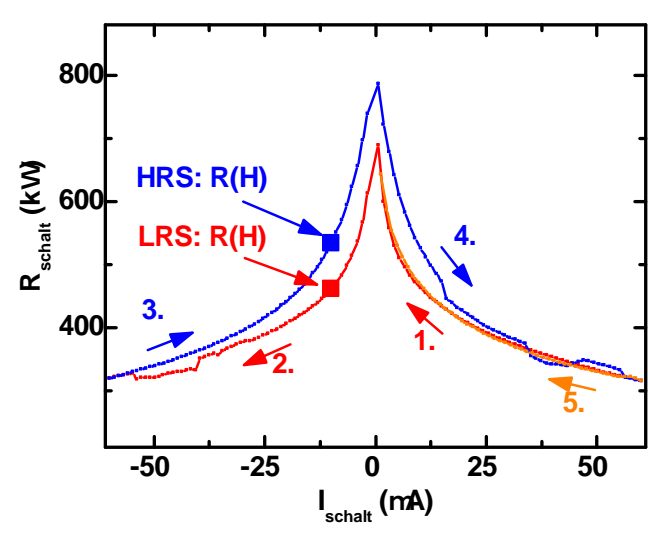

(a) elektrisches Widerstandsschalten

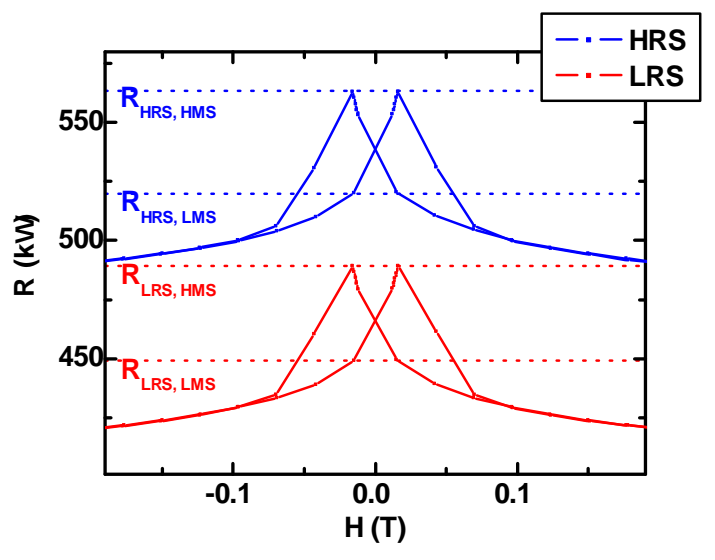

(b) LFMR in HRS und LRS

Abb. 6.12: Kombination von Magnetowiderstands- und elektrischen Widerstandsschaltmessungen $(T=10 \mathrm{~K}$, Elektrodenabstand $a=1 \mu \mathrm{m}$, Stegbreite $b=5 \mu \mathrm{m}$, $\mathrm{LSMO} / \mathrm{Al}_{2} \mathrm{O}_{3}$, (012)-orientiert). Sowohl im HRS als auch im LRS wird ein LFMR beobachtet (b). Daraus resultieren vier verschiedene Widerstandswerte $\left(R_{\mathrm{HRS}, \mathrm{HMS}}, R_{\mathrm{HRS}}\right.$, LMS,$R_{\mathrm{LRS}}$, HMS und $R_{\mathrm{LRS}}$, LMS $)$, die durch eine Kombination aus elektrischen und magnetischen Feldern remanent eingestellt werden können. 


\subsubsection{Einfluss der Temperatur}

Im nachfolgenden Abschnitt wird der Einfluss der Temperatur auf das Widerstandsschalten in den Mikrostrukturen untersucht. In Abb. 6.13 sind Widerstandsschaltcharakteristiken, die bei verschiedenen Temperaturen aufgenommen wurden, abgebildet. Auch in diesen Messungen ist die systematische Variationsgröße der Schaltstrom. In Abb. 6.13 sind die einzelnen Messabschnitte einer Hysterese $\left(I_{\text {schalt }}: 0 \rightarrow+I_{\max } \rightarrow-I_{\max } \rightarrow+I_{\max } \rightarrow 0\right)$ farblich gekennzeichnet: Für die Kennlinie von $0 \rightarrow+I_{\max }$ wird Schwarz, für $+I_{\max } \rightarrow$ $-I_{\max }$ Rot, für $-I_{\max } \rightarrow+I_{\max }$ Grün und für $+I_{\max } \rightarrow 0$ Blau verwendet.

In Abb. 6.13(a) ist die Schaltkennlinie bei $T=10 \mathrm{~K}$ gezeigt. Der Widerstand schaltet bei positiven Strömen von einem hochohmigen in einen tiefohmigen Zustand, bei negativen Strömen ist der (Haupt)-Schaltvorgang von LRS nach HRS. Diese Charakteristik wird auch in den Messungen in Abb. 6.13(a) bis (d) beobachtet, die bei $T=10 \ldots 180 \mathrm{~K}$ aufgenommen wurden. Die kritischen Schaltströme bzw. -spannungen sind jeweils mit einem roten Pfeil gekennzeichnet. Im Bereich negativer Schaltströme ist in einigen Messungen ((a), (b), (d) und (h)) ein zweiter Schaltprozess zu sehen. Dieser ist mit blauen Pfeilen markiert. Insgesamt ändern sich die Schaltcharakteristiken nicht wesentlich in den Messungen (a) bis (d), in denen die Temperatur von $T=10 \mathrm{~K}$ auf $180 \mathrm{~K}$ angehoben wurde. Für die kritischen Schaltströme bzw. die kritischen Schaltspannungen (entsprechend der roten Pfeile in Abb. 6.13) ergibt sich als Mittelwert $\bar{U}_{\mathrm{c}, \mathrm{HRS} \rightarrow \mathrm{LRS}}=10,6 \mathrm{~V} \pm 8 \%$ bzw. $\bar{I}_{\mathrm{c}, \mathrm{HRS}} \rightarrow$ LRS $=22 \mu \mathrm{A} \pm 9 \%$. Für den umgekehrten Schaltvorgang wird $\bar{U}_{\mathrm{c}}$, LRS $\rightarrow$ HRS $=$ $7,5 \mathrm{~V} \pm 7 \%$ bzw. $\bar{I}_{\mathrm{c}}$, LRS $\rightarrow$ HRS $=13 \mu \mathrm{A} \pm 9 \%$ beobachtet.

Die Messungen (a),(f) und (h) wurden alle bei einer Temperatur von $T=10 \mathrm{~K}$ aufgenommen. Hier zeigt sich, dass in diesen Messungen die Variation des kritischen Schaltstroms (und auch der hieraus berechneten kritischen Schaltspannung) genauso groß ist wie in den Messungen, die bei unterschiedlichen Temperaturen aufgenommen worden sind. Daher ist keine sinnvolle Analyse der kritischen Schaltspannungen und Schaltströme als Funktion der Temperatur möglich. Es kann lediglich festgehalten werden, dass für Temperaturen zwischen $T=10 \ldots 180 \mathrm{~K}$ qualitativ gleiche Kennlinien mit den gleichen Schaltprozessen beobachtet werden.

Bei $T=275 \mathrm{~K}$ (Abb. 6.13(e)) verändert sich die Form der $R(I)$-Kennlinie drastisch: Der erste (in schwarz dargestellte) Messabschnitt $\left(I_{\text {schalt }}: 0 \rightarrow+I_{\text {max }}\right)$ zeigt zu Beginn einen hochohmigen Widerstandszustand. Im Laufe dieses Abschnitts wird der Strom immer weiter erhöht, bis der Widerstand schließlich aufgrund eines Strom-compliances nicht mehr gemessen werden kann. Im nächsten Messpunkt ist der Widerstand in einem sehr tiefohmigen Zustand, dieser ist durch den schwarzen Pfeil 2 markiert. Dieser erste, drastische Schaltprozess wird nur einmal beobachtet. Auf dem Weg von $+I_{\max } \rightarrow-I_{\max }$ (rote Messdaten) ist keine sprunghafte Veränderung des Widerstandes zu sehen, allerdings ist die Kennlinie stark asymmetrisch. Die asymmetrische Kennlinie bleibt auch für $-I_{\max } \rightarrow+I_{\max }$ (grüne Datenpunkte) erhalten. Es ist allerdings ein (kleiner) Unterschied zwischen den roten und grünen Messpunkten zu sehen, der bei größeren Strömen geringer ist. In dem Messabschnitt von $+I_{\max } \rightarrow 0$ liegen die Messdaten (blau) wieder weitestgehend auf der roten Kurve.

Um zu überprüfen, ob eine irreversible Änderung der Struktur stattgefunden hat, wurde daraufhin die Probe wieder auf $T=10 \mathrm{~K}$ abgekühlt. In der in Abb. 6.13(f) gezeigten Kennlinie ist die starke Asymmetrie der in (e) abgebildeten Messung nicht mehr vorhanden, die Kurve stimmt mit den vorherigen Messungen bei Temperaturen kleiner als $275 \mathrm{~K}$ überein. Nach der Kurve in (f) bei $T=10 \mathrm{~K}$ wird die Probe wieder auf eine Temperatur von $210 \mathrm{~K}$ 


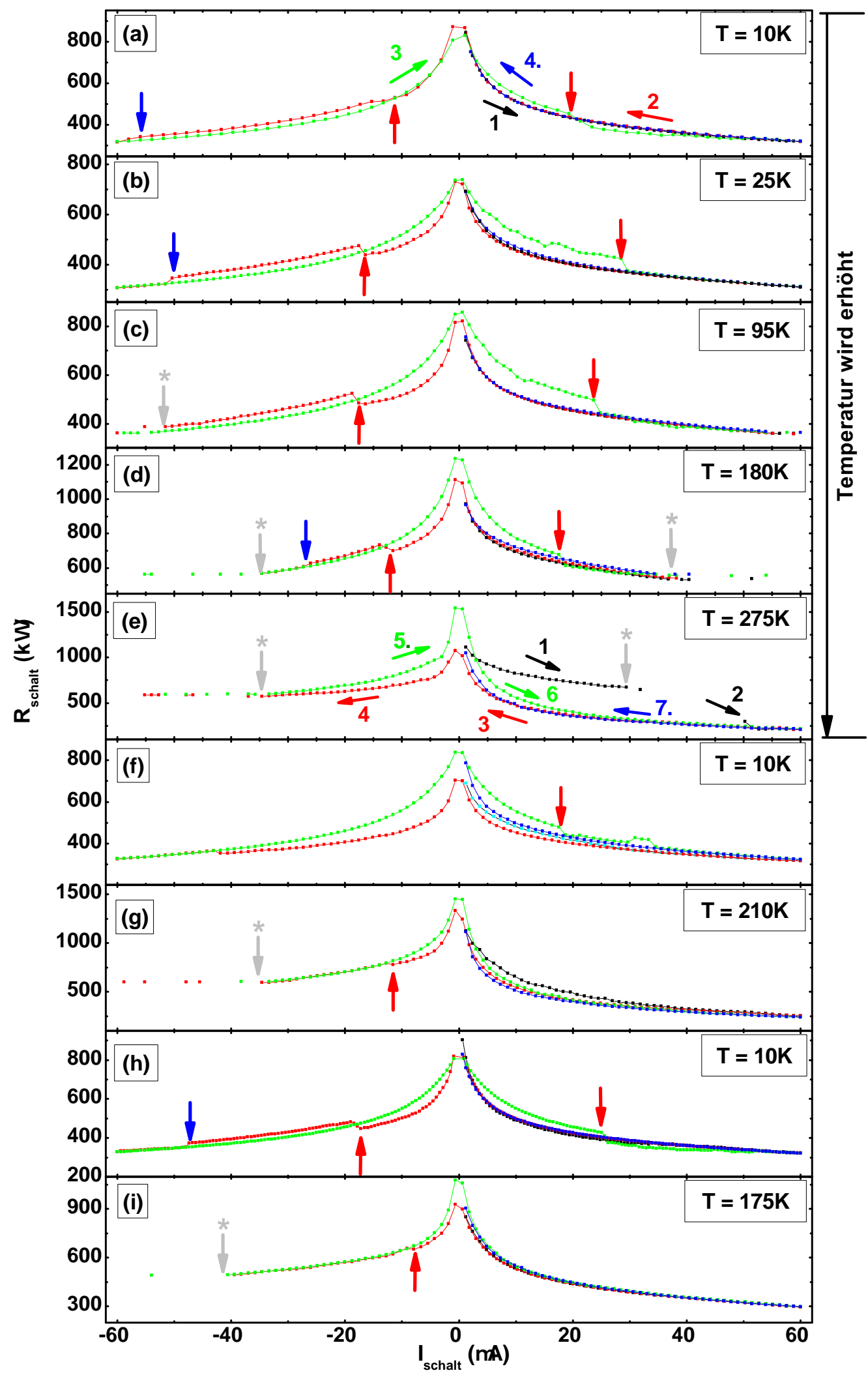

Abb. 6.13: Einfluss der Temperatur auf das Widerstandsschalten $(d=1 \mu \mathrm{m}, b=5 \mu \mathrm{m}$, $\mathrm{LSMO} / \mathrm{Al}_{2} \mathrm{O}_{3}$ (012) orientiert). Die Messungen wurden nacheinander von (a) nach (i) aufgenommen. Die roten und blauen Pfeile zeigen die kritischen Schaltströme bzw. -spannungen. In Grau sind die Bereiche, die aufgrund eines Stromcompliances nicht angegeben werden können, markiert. 


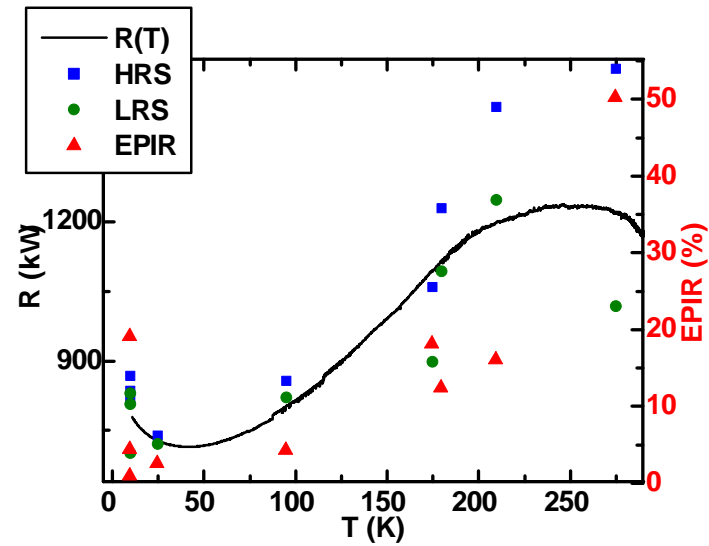

(a) $R(T)$ mit HRS, LRS und EPIR

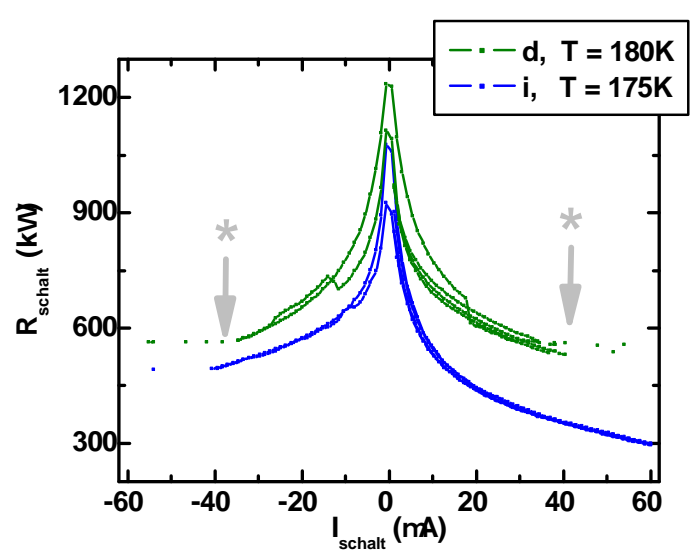

(b) Vergleich der Messungen (d) und (i)

Abb. 6.14: (a) Temperaturabhängigkeit des Widerstands (im LRS, $I=1 \mu \mathrm{A}$ ) mit HRS, LRS und EPIR aus Abb. $6.13(I=1 \mu \mathrm{A})$. (b) Vergleich der Messungen (d) und (i) aus Abb. 6.13.

aufgeheizt. Dieses ist in Abb. 6.13(g) gezeigt. Die Schaltcharakteristik ist in dieser Messung von Anfang an asymmetrisch, der drastische Abfall, der in Messung (e) beobachtet wird, fehlt. Ein weiteres Abkühlen auf $10 \mathrm{~K}$ zeigt wieder die für tiefe Temperaturen typischen, symmetrischen Kurven. In der nachfolgenden Messung (Abb. 6.13(i)) wird die Asymmetrie bei $175 \mathrm{~K}$ beobachtet, also bei einer Temperatur, bei der zuvor (Abb. 6.13(d)) die Kurve symmetrisch war. Ein Vergleich der Messungen (d) und (i) ist in Abb. 6.14(b) gezeigt.

Asymmetrische Kennlinien wurden in einigen Proben und Mikrostrukturen bei höheren Temperaturen beobachtet. Dabei ist die Temperatur, ab der dieses Verhalten auftritt, unterschiedlich für verschiedene Strukturen und Proben.

In Abb. 6.14(a) ist die Temperaturabhängigkeit des Widerstands zu sehen (der Widerstand wurde mit $I=1 \mu \mathrm{A}$ gemessen). R(I)-Kennlinien vor und nach dieser Messungen zeigten, dass die Struktur im LRS war. Zusätzlich sind in Abb. 6.14(a) die verschiedenen Widerstandswerte (HRS und LRS) aus den Schaltcharakteristiken der Abb. 6.13 eingetragen. HRS und LRS wurden dabei für die Messungen (a) bis (h) bei $I=1 \mu \mathrm{A}$ bestimmt. Des Weiteren sind in der Abb. 6.14(a) die zugehörigen EPIR-Beträge als Funktion der Temperatur eingezeichnet. Alle diese Werte streuen stark; daher ist es schwierig, eine systematische Temperaturabhängigkeit zu erkennen. In anderen Worten: Der Einfluss der Temperatur auf die Schaltcharakteristiken ist kleiner als die Schwankungen aufeinanderfolgender Messungen bei konstanter Temperatur (hierbei wird die Asymmetrie nicht berücksichtigt).

Die temperaturabhängigen Messungen demonstrieren, dass zwischen 10 . . $180 \mathrm{~K}$ das Widerstandsschalten im Wesentlichen unverändert ist (Abb. 6.13(a) bis (d)). Weitere Messungen (an einer anderen Mikrostruktur) zeigen, dass sich auch zwischen $T=2 \ldots 10 \mathrm{~K}$ die Schaltcharakteristiken qualitativ nicht verändern (daher wird hier auf eine Abbildung verzichtet). Bei hohen Temperaturen treten starke Asymmetrien auf, die bei verschiedenen Proben ab einer unterschiedlichen Temperatur beobachtet werden. Trotz der Assymetrie können in den Kennlinien ähnliche Schaltcharakteristiken beobachtet werden, wie sie in den Messungen bei tiefen Temperaturen gefunden werden. 


\subsection{Diskussion}

Die Abschnitte 6.1 und 6.2 stellen die Messergebnisse der Widerstandsschaltexperimente vor. Ein wichtiges Ergebnis ist die bevorzugte Lokalisierung des Widerstandsschaltens im Bereich der Korngrenzen. Dieser Einfluss der Korngrenzen auf das elektrische Widerstandsschalten wird im Rahmen der nachfolgenden Diskussion analysiert.

Als erstes werden im Abschnitt 6.3.1 einige energetische Betrachtungen vorgestellt, wobei die Energieniveaus des hoch- und niederohmigen Zustands in einem Doppelmuldenpotential beschrieben werden. Anschließend (Abschnitt 6.3.2) werden Literaturergebnisse an sehr unterschiedlichen Materialsystemen vorgestellt, in denen ebenfalls der Einfluss von höherdimensionalen Defekten auf das Widerstandsschalten untersucht wurde. Hierbei werden die Gemeinsamkeiten und Unterschiede zu den C-AFM Messungen des Abschnitts 6.1 aufgezeigt.

Im Abschnitt 6.3.3 folgt eine Beschreibung der Mikrostrukturmessungen in Form eines Widerstandsnetzwerks aus Manganatsäulen. Im Rahmen dieser Beschreibung können viele Messergebnisse qualitativ simuliert werden. Anschließend werden die temperatur- und magnetfeldabhängigen Mikrostrukturmessungen diskutiert.

Auf diesen Grundlagen werden schließlich die Ergebnisse in Bezug auf den mikroskopischen Mechanismus, der dem Widerstandsschalten zugrunde liegt, im Abschnitt 6.3.4 zusammengefasst und ausgewertet. Dabei wird das Modell eines lokalen strukturellen Übergangs dem der Sauerstoffleerstellen-Diffusion gegenübergestellt.

\subsubsection{Energetische Betrachtungen}

Zunächst werden die vorgestellten Messergebnisse im Rahmen eines Doppelmuldenpotentials in einer verallgemeinerten Energielandschaft zusammengefasst. Dabei wird noch nicht auf den zugrunde liegenden mikroskopischen Schaltmechanismus eingegangen. Das bipolare Widerstandsschalten ist remanent und reversibel, entsprechend müssen in der Energielandschaft zwei lokale Minima existieren, die dem HRS und LRS zugeordnet werden können. Durch ein genügend hohes elektrisches Feld kann die Potentialbarriere zwischen den Widerstandszuständen überwunden werden. Entsprechende Doppelmuldenpotentiale sind in Abb. 6.15 dargestellt. Diese wurden in den Ref. [60] und [88] eingeführt und werden im Folgenden erweitert.

Ein Ergebnis der Mikrostrukturmessungen ist es, dass zwischen dem HRS und LRS weitere Widerstandszustände liegen können. Da diese Zustände remanent sind, müssen entsprechende Minima in der Energielandschaft vorhanden sein. ${ }^{4}$ Diese werden in Abb. 6.15 nicht berücksichtigt, da die Zwischenzustände stark abhängig beispielsweise von der jeweiligen Mikrostruktur sind. Daher sind keine allgemeinen Aussagen zu den Zwischenzuständen möglich. In Abb. 6.15 werden für die Oberflächen (Abb. 6.15(a)) und die Korngrenzen (Abb. 6.15(b)) unterschiedliche Potentiale gezeigt, wie nachfolgend erläutert wird. Auf die veränderte Energielandschaft an einer Korngrenze bei der $300 \mathrm{~nm}$ dicken, nanokolumnaren Schicht (Abb. 6.15(c)) wird am Ende dieses Abschnittes eingegangen.

Nachfolgend werden die vorgestellten Messergebnisse in Bezug auf die in Abb. 6.15 gezeigten Energiebarrieren und Energieniveaus für das Widerstandsschalten an der Oberfläche

\footnotetext{
${ }^{4}$ Die Zwischenzustände können dabei durch das Schalten an den unterschiedlichen Körnern und auch durch das Wachstum von LRS-Bereichen entlang der Korngrenzen von einzelnen Säulen verursacht sein, wie in den C-AFM Messungen des Abschnitts 6.1.2 zu sehen ist.
} 
(a) Oberfläche

(b) Korngrenze

(c) Korngrenze
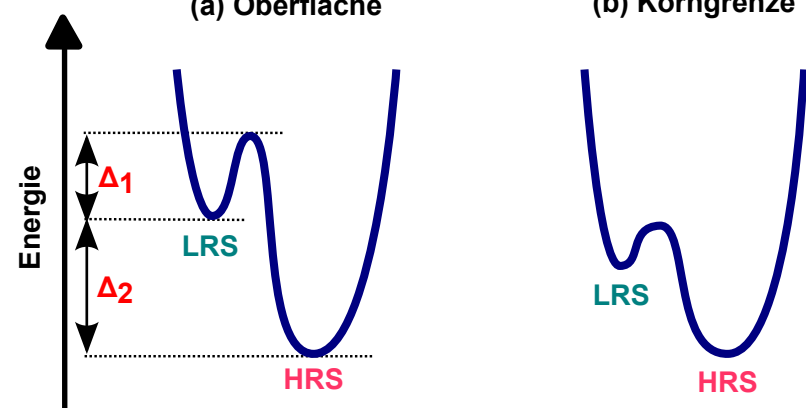

(dicke Schicht)

verallgemeinerte Koordinate

Abb. 6.15: Doppelmuldenpotentiale zur Darstellung der Energieniveaus von HRS und LRS. Gezeigt ist eine Potentiallandschaft für eine Manganatoberfläche (a), für Korngrenzen von $40 \ldots 60 \mathrm{~nm}$ (b) und einer $300 \mathrm{~nm}$ dicken, nanokolumnaren Schicht (c). Die absoluten Energieniveaus der HRS- und LRS-Zustände können nicht angegeben werden, im Text finden sich aber quantitative Aussagen für die relative Größe der Energiebarrieren $\Delta_{1}$ und $\Delta_{2}$.

und im Bereich der Korngrenze untersucht. Hierdurch ist es möglich, qualitative und quantitative Aussagen zu der relativen Lage der Energieniveaus und -barrieren zu treffen. Wenn nicht anders gekennzeichnet, beziehen sich die folgenden Aussagen auf die nanokolumnaren Proben mit Schichtdicken zwischen $40 \ldots 60 \mathrm{~nm}$. Dabei ist zu beachten, dass die C-AFM Messungen bei Raumtemperatur aufgenommen wurden, die Mikrostrukturmessungen bei Temperaturen meist unterhalb von $T=100 \mathrm{~K}$.

\section{- HRS energetisch günstiger als LRS}

Der Ausgangszustand der C-AFM Messungen zeigt eine homogene Verteilung des HRS. Unter der Annahme, dass der Ausgangszustand des Systems dem Gleichgewichtszustand entspricht, ist der HRS in den vorgestellten Messungen energetisch günstiger als der LRS. Dieses gilt sowohl für die Manganatoberfläche als auch für den Bereich der Korngrenzen (Schichtdicken zwischen $40 \ldots 60 \mathrm{~nm}$ ).

Des Weiteren zeigen die Mikrostrukturmessungen, dass die Schaltspannungen für das Schalten von HRS nach LRS (fast) immer größer als für das Schalten von LRS nach HRS sind. Für das Verhältnis zwischen den kritischen Schaltspannungen $U_{\mathrm{c}}^{*}=$

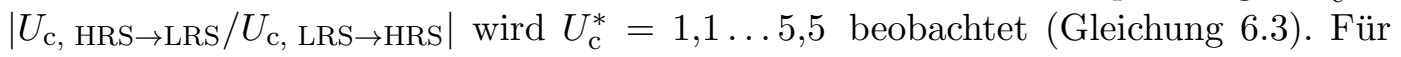
die Energiebarrieren $\Delta_{1}$ und $\Delta_{2}$ (Abb. 6.15) ergibt sich daher

$$
\frac{\Delta_{1}+\Delta_{2}}{\Delta_{1}} \triangleq\left|\frac{U_{\mathrm{c}, \mathrm{HRS} \rightarrow \mathrm{LRS}}}{U_{\mathrm{c}, \mathrm{LRS} \rightarrow \mathrm{HRS}}}\right|=1,1 \ldots 5,5
$$

Dieses Ergebnis gilt für nanokolumnare Proben mit Schichtdicken von $40 \ldots 60 \mathrm{~nm}$. Da $U_{\mathrm{c}}^{*}$ aus den Mikrostrukturmessungen bestimmt wurde, kann nicht unterschieden werden, ob es sich um die Manganatoberfläche oder den Bereich der Korngrenzen handelt.

\section{- Kritische elektrische Feldstärken}

Die C-AFM Messungen (Abb. 6.1) zeigen in unmittelbarer Nähe (bis zu einem Abstand von etwa $40 \mathrm{~nm}$ ) der mit $U_{\text {schalt }}$ gerasterten Linien keine große Korrelation mit der Topographie. Hier schalten sowohl die Korngrenzen als auch die Oberflächen der 
einzelnen Körner. Diese Beobachtung ist konsistent mit den Messungen an epitaktischen Proben, in denen das Widerstandsschalten an der Manganatoberfläche direkt unterhalb der Cantileverspitze stattfindet. [60] Im Gegensatz dazu findet sich in größerer Entfernung der LRS bevorzugt im Bereich der Korngrenzen. Der maximale Abstand $r$ (zwischen den in den LRS geschalteten Bereichen der Korngrenzen und der Stelle, in der $U_{\text {schalt }}$ angelegt wurde) liegt bei $650 \mathrm{~nm}$ und resultiert aus der Größe des Bildausschnitts der C-AFM Messungen. Dieses unterschiedliche Schaltverhalten kann durch eine von dem Abstand $r$ abhängige, elektrische Feldstärke verstanden werden.

Wird angenommen, dass das elektrische Feld in der Nähe der Cantileverspitze eine radiale Feldverteilung zeigt, so ist die elektrische Feldstärke $E \propto \frac{U}{r}$. Eine radiale Feldverteilung scheint plausibel, da die LRS-Bereiche in alle Richtungen von der mit $U_{\text {schalt }}$ gemessenen Stelle zu finden sind. Es wird zumindest innerhalb des Messausschnittes $^{5}$ zu der erhöhten Spannung kein einzelner LRS-Pfad beobachtet.

Allerdings ist zu beachten, dass für die nanokolumnaren Manganatschichten eigentlich keine homogene Feldverteilung zu erwarten ist. Die Annahme einer homogenen radialen Feldverteilung dient lediglich dazu, eine grobe Abschätzung der elektrischen Feldstärke in Abhängigkeit des Abstands $r$ zu ermöglichen. Die Oberfläche der Manganatschicht schaltet in einem maximalen Abstand von $r \approx 40 \mathrm{~nm}$, im Gegensatz dazu finden sich LRS-Bereiche in den Korngrenzen in über $500 \mathrm{~nm}$ Entfernung.

Im Rahmen dieser groben Abschätzung kann das Verhältnis der elektrischen Feldstärken, die ein Schalten induzieren, mit

$$
\frac{E_{\text {Oberfläche }}}{E_{\text {Korngrenze }}} \approx \frac{500 \mathrm{~nm}}{40 \mathrm{~nm}}=12,5 \approx \frac{\left(\Delta_{1}+\Delta_{2}\right)_{\text {Oberfläche }}}{\left(\Delta_{1}+\Delta_{2}\right)_{\text {Korngrenze }}}
$$

angegeben werden. Hier ist also das elektrische Feld, das benötigt wird, um eine Manganatoberfläche zu schalten, um mindestens einen Faktor von 12,5 größer als im Bereich einer Korngrenze. Dieses entspricht dem Verhältnis der jeweiligen Energiebarrieren $\frac{\left(\Delta_{1}+\Delta_{2}\right)_{\text {Oberfläche }}}{\left(\Delta_{1}+\Delta_{2}\right)_{\text {Korngrenze }}}$.

\section{- Kritische Schaltspannungen}

Ein weiterer wichtiger Aspekt bei dem Vergleich der Schalteigenschaften von Manganatoberflächen und Korngrenzen betrifft die kritischen Schaltspannungen. Bei den epitaktischen Schichten ist eine Schaltspannung von mindestens $3 \mathrm{~V}$ nötig, um das Widerstandsschalten an den Oberflächen zu initiieren. [60]

Diese kritische Schaltspannung ist für die nanokolumnaren Schichten niedriger. In der in Abb. 6.5 gezeigten Messung wurde wiederholt derselbe Probenbereich mit einer Spannung von $U_{\text {mess }}=0,8 \mathrm{~V}$ abgerastert. Im Laufe der Messungen nimmt die Größe und Anzahl der LRS-Bereiche (entlang der Korngrenzen) zu. Demnach ist die kritische Schaltspannung in den epitaktischen Proben mindestens um einen Faktor von etwa 3,75 größer als in den nanokolumnaren Schichten.

Aus dieser Beobachtung ergibt sich für das Verhältnis der Energiebarrieren in der Oberfläche und in den Korngrenzen

$$
\frac{\left(\Delta_{1}+\Delta_{2}\right)_{\text {Oberfläche }}}{\left(\Delta_{1}+\Delta_{2}\right)_{\text {Korngrenze }}} \geq 3,75
$$

\footnotetext{
${ }^{5}$ Der Messausschnitt hat eine Kantenlänge von $1 \mu \mathrm{m}$; in diesem Bereich wird kein bevorzugtes Schalten
} in einer Richtung beobachtet. 
Dieses stimmt mit Gleichung 6.7 überein. Dabei ist zu beachten, dass in Gleichung 6.7 die Oberfläche und Korngrenze einer polykristallinen Schicht und in Gleichung 6.8 der Unterschied zwischen einer epitaktischen und nanokolumnaren Schicht (unterschiedlicher Dotierung) verglichen werden.

\section{- Abhängigkeit von der Schichtdicke}

C-AFM Messungen (Abb. 6.7) an einer dickeren Schicht (300 nm) zeigen, dass schon bei der ersten Aufnahme mit einer Messspannung von lediglich $U_{\text {mess }}=0,1 \mathrm{~V}$ wesentliche Bereiche der Korngrenzen im LRS sind. Wird angenommen, dass dieses dem Ausgangszustand entspricht, ist hier der LRS energetisch günstiger als der HRS. Dieses wird in Abb. 6.15(c) durch eine zusätzliche Potentiallandschaft für die $300 \mathrm{~nm}$ dicke, nanokolumnare Schicht verdeutlicht. Ein weiterer Erklärungsansatz ist, dass die Energiebarriere $\left(\Delta_{1}+\Delta_{2}\right)$ in dieser Schicht so weit reduziert ist, dass mit einer Messspannung von nur $U_{\text {mess }}=0,1 \mathrm{~V}$ schon in den LRS-Zustand geschaltet wird. Auf Grundlage der vorhandenen Messdaten können diese beiden Szenarien nicht unterschieden werden. Eindeutig ist allerdings, dass der LRS im Bereich der Korngrenzen für die dickere Schicht energetisch wesentlich günstiger als für die dünne Schicht ist.

Es ist zu beachten, dass die vorhandenen Messungen keine Aussage über die Absolutwerte der Energieniveaus von HRS und LRS weder an der Schichtoberfläche noch an den Korngrenzen erlauben.

Eine weitere interessante Ergänzung zu den in Abb. 6.15 gezeigten Potentiallandschaften betrifft die C-AFM Untersuchungen von Krisponeit an einer epitaktischen $\mathrm{La}_{0,7} \mathrm{Ca}_{0,3} \mathrm{MnO}_{3}$ Schicht, die eine etwa zwei Monolagen dicke $\mathrm{LaMnO}_{3}$-Decklage hat. [60] Diese Probe zeigt im Ausgangszustand beinahe flächendeckend eine metallische Leitfähigkeit. Entsprechend ist dort ähnlich wie bei der $300 \mathrm{~nm}$ dicken $\mathrm{LSMO} / \mathrm{Al}_{2} \mathrm{O}_{3}$-Probe der LRS an der Oberfläche energetisch begünstigt.

\subsubsection{Literaturvergleich: Einfluss von höherdimensionalen Defekten}

Der Einfluss von höherdimensionalen Defekten wie Versetzungen und Korngrenzen auf das bipolare Widerstandsschalten wird in einer zunehmenden Anzahl von aktuellen Veröffentlichungen an unterschiedlichen Oxiden untersucht. Eine herausragende Rolle bei diesen Studien spielen C-AFM Experimente, in denen der Einfluss der lokalen Defektstruktur auf die Verteilung der unterschiedlich leitfähigen Bereiche untersucht werden kann. In den meisten Arbeiten wird allerdings eine andere Messgeometrie als die hier benutzte verwendet. Die oxidischen Schichten werden auf leitfähigen Substraten bzw. Schichten deponiert; diese dienen (neben der Cantileverspitze) als zweite Elektrode (Sandwich-Geometrie, siehe Abb. 3.1(a)D. Da die zweite Elektrode sich also unter der oxidischen Schicht befindet, erlaubt diese Geometrie weniger Rückschluss auf die Verteilung der schaltenden Bereiche in der oxidischen Schicht. So bedeutet etwa eine variierende Schichtdicke eine unterschiedlich dicke Barriere zwischen den Elektroden. Dieses kann insbesondere bei sehr dünnen Schichten einen Einfluss auf die lokalen Schaltparameter haben.

Lanza et al. stellen C-AFM Messungen an polykristallinem $\mathrm{HfO}_{2}$ in Sandwich-Geometrie vor. [106] Sie beobachten, dass das Widerstandsschalten von Filamenten, die hauptsächlich in den Korngrenzen gebildet werden, getragen wird. C-AFM Untersuchungen an polykristallinen NiO-Filmen, wiederum in Sandwich-Geometrie, werden von Son et al. vorgestellt. [55] In diesen Schichten finden sich schon im Ausgangszustand leitfähige Filamente, welche im Bereich der Korngrenzen lokalisiert sind. Nachdem die Probe in den LRS 
geschaltet wurde, findet sich eine erhöhte Anzahl von leitfähigen Filamenten, die sowohl innerhalb der Korngrenzen als auch unmittelbar auf den Körnern zu finden sind.

Des Weiteren gibt es interessante C-AFM Untersuchungen an dem perowskitischen $\mathrm{SrTiO}_{3}$ System (in Sandwich-Geometrie): Szot el al. beobachten, dass das Widerstandsschalten im Bereich von Versetzungen stattfindet. [19] Die mikroskopische Ursache für das Schalten wird in einer Sauerstoffleerstellen-Diffusion gesehen, wobei die Sauerstoffvakanzen entlang der Versetzungen diffundieren bzw. in diesen ein erhöhter Massentransport möglich ist (Stichwort: pipe like diffusion). [19] Eine weitere C-AFM Arbeit an $\mathrm{SrTiO}_{3}$-Schichten (ebenfalls in Sandwich-Geometrie) untersucht den Einfluss von unterschiedlichen Defektstrukturen. [107] In lagenweise gewachsenen Schichten (layer-by-layer growth) sind die Bereiche, in denen der Widerstand geschaltet wird, unregelmäßig verteilt und bestehen aus kleinen Punkten (mit einem Durchmesser von $1 \ldots 2 \mathrm{~nm}$ ). Diese werden wie in der Arbeit von Szot et al. mit Versetzungen in Verbindung gebracht. TEM Messungen an weiteren Schichten fanden eine ähnliche Defektstruktur wie die in dieser Arbeit untersuchten, nanokolumnaren Manganatschichten. Auch in diesen $\mathrm{SrTiO}_{3}$-Schichten zeigten C-AFM Messungen, dass das Widerstandsschalten bevorzugt an den Korngrenzen zwischen den einzelnen Körnern lokalisiert ist. Die resultierenden Stromkarten haben eine große Ähnlichkeit zu den Stromkarten in Abb. 6.1(c). Muenstermann et al. erklären ihre Ergebnisse ebenfalls im Rahmen einer Sauerstoffleerstellen-Diffusion. [107] Dabei fungieren die defektreichen Probenbereiche also insbesondere die Korngrenzen als Kanäle für eine erhöhte Diffusion.

Auch zu den Manganaten gibt es einige wenige Untersuchungen: Eine der ersten C-AFM Messungen wurde von Chen et al. [108] an polykristallinen $\mathrm{La}_{0,8} \mathrm{Sr}_{0,2} \mathrm{MnO}_{3}$-Schichten durchgeführt. Dabei wurde eine Iridium-Schicht als untere Elektrode verwendet. Ein Ergebnis dieser Messungen ist, dass die LRS-Bereiche eine granulare Verteilung zeigen. Diese Verteilung korreliert mit der Topographie, die submikrometer große Inseln an der Probenoberfläche zeigt. Daher ergibt sich eine gute Übereinstimmung mit den Ergebnissen der vorliegenden Arbeit. Es ist allerdings zu beachten, dass Chen et al. in Sandwich-Geometrie messen.

C-AFM Messungen an epitaktischen $\mathrm{La}_{0,7} \mathrm{Sr}_{0,3} \mathrm{MnO}_{3}$-Schichten auf $\mathrm{SrTiO}_{3}$-Substraten werden von Moreno et al. vorgestellt. [109] In ihrer Untersuchung wurde ein planarer Messaufbau verwendet (isolierendes Substrat und zweite Elektrode am Probenrand). Auch hier wird im C-AFM bipolares Schalten initiiert. Es gibt allerdings zwischen der Arbeit von Moreno et al. und den hier vorgestellten Ergebnissen einige Unterschiede: Der Ausgangszustand der $\mathrm{La}_{0,7} \mathrm{Sr}_{0,3} \mathrm{MnO}_{3} / \mathrm{SrTiO}_{3}$-Schicht ist der LRS, dieser schaltet bei einer positiven Spannung $(+4 \mathrm{~V}$, tip voltage $)$ in den HRS. Bei einer negativen Spannung kann der LRS-Zustand wiederhergestellt werden, wobei die negative Schaltspannung (HRS $\rightarrow$ LRS) größer als die positive Schaltspannung (LRS $\rightarrow$ HRS) ist. Der Ausgangszustand der Probe und die Polarität der Schaltspannung sind damit genau umgekehrt zu den Ergebnissen von Chen et al. [108] und den hier vorgestellten Messungen. Eine Ursache für diese unterschiedlichen Messresultate kann an dieser Stelle nicht gefunden werden. Eine kleine Übereinstimmung zwischen den Ergebnissen von Moreno et al. [109] und dieser Arbeit gibt es in Bezug auf das veränderte Schaltverhalten in der Nähe von höherdimensionalen Defekten. In den Proben von Moreno et al. finden sich an der Oberfläche Nanometer kleine Ausscheidungen von La-Sr-Oxiden. [109] In der Nähe dieser Ausscheidungen wird zum Wiederherstellen des LRS eine etwas geringere (negative) Spannung als im Rest der Probe benötigt. In anderen Worten: die Defektstruktur erniedrigt die kritische Schaltspannung. 
Höherdimensionale Defekte zeigen also in sehr unterschiedlichen Probensystemen einen ähnlichen Einfluss auf das Schaltverhalten wie es in der hier vorgestellten Untersuchung für nanokolumnare Manganatschichten beobachtet wird. In vielen Probensystemen wird eine niedrigere Schaltspannung im Bereich der Korngrenzen gefunden. Es wird beschrieben, dass das Schalten bevorzugt im Bereich der Korngrenzen und Versetzungen stattfindet, entsprechend der Überlegungen im Abschnitt 6.3.1. Nach diesem Literaturvergleich der CAFM Messungen werden im nachfolgenden Abschnitt die Mikrostrukturmessungen diskutiert. Auf den zugrunde liegenden mikroskopischen Mechanismus des Widerstandsschaltens wird im Abschnitt 6.3.4 eingegangen.

\subsubsection{Mikrostrukturen: Widerstandsnetzwerke aus Manganatsäulen}

Im Folgenden werden die Widerstandskennlinien der Mikrostrukturen im Rahmen eines Widerstandsnetzwerkes diskutiert. Bei der Interpretation der Schaltkennlinien muss eine ganze Reihe von Widerstandsbeiträgen beachtet werden. Alle Elemente, die zum Gesamtwiderstand in den Mikrostrukturmessungen beitragen, werden zur besseren Übersicht an dieser Stelle kurz aufgelistet und sind in Abb. 6.16(a) in Form eines schematischen Widerstandsnetzwerks gezeigt.

\section{- Die Manganatsäulen}

Die strukturierte Manganatbrücke ist typischerweise einige $\mu \mathrm{m}^{2}$ groß, während die Korndurchmesser der Manganatsäulen (in Abhängigkeit von der Schichtdicke) zwischen $40 \ldots 130 \mathrm{~nm}$ variieren. Bei der kleinsten untersuchten Strukturgröße $(0,8 \times$ $1,3 \mu \mathrm{m})$ ergibt sich daraus ein Widerstandsnetzwerk aus etwa $16 \times 26$-Manganatsäulen. Die Manganatsäulen sind sowohl parallel als auch in Reihe geschaltet. Da die C-AFM Messungen zeigen, dass das Widerstandsschalten bevorzugt an den Korngrenzen stattfindet, bietet es sich an, den Widerstand einer Manganatsäule aus zwei Komponenten zusammenzusetzen: Zum einen dem Widerstand des Korninneren

$\mathrm{R}_{\text {Säule }}$ und zum zweiten einem Beitrag der Korngrenze $\mathrm{R}_{\text {Korngrenze }}$, dem ein bipolares Widerstandsschalten zugeordnet wird. Diese Einteilung in den Bereich der Korngrenzen bzw. -hülle und des Korninneren entspricht dem in Abschnitt 5 zur Erklärung der elektronischen und magnetischen Transporteigenschaften der $\mathrm{La}_{0,7} \mathrm{Sr}_{0,3} \mathrm{MnO}_{3} / \mathrm{Al}_{2} \mathrm{O}_{3^{-}}$ Proben eingeführten core shell-Modell.

\section{- Kontaktwiderstand zwischen Elektroden und Manganatschicht}

Auch die Kontaktwiderstände zwischen der Manganatschicht und den Elektroden tragen zum Gesamtwiderstand der Struktur bei. Wie in Kap. 3 beschrieben wird, zeigen viele Veröffentlichungen, dass das Widerstandsschalten hier lokalisiert ist bzw. initiiert wird. In den obigen energetischen Betrachtungen (Abschnitt 6.3.1) wird für die nanokolumnaren Schichten gezeigt, dass für das Widerstandsschalten an den Korngrenzen kleinere elektrische Felder als an der Schichtoberfläche notwendig sind.

Esseling führte Vergleichsmessungen zwischen nanokolumnaren und epitaktischen Manganatschichten durch und fand ausschließlich in den nanokolumnaren LSMOProben Widerstandsschalten. Hiermit wurde die Bedeutung der Korngrenzen für den Schaltprozess aufgezeigt. [88] Auch Untersuchungen in $\mathrm{La}_{0,67} \mathrm{Sr}_{0,33} \mathrm{MnO}_{3} / \mathrm{LaAlO}_{3}$ von Xie et al. zeigen, dass eine hohe Schichtrauigkeit das Widerstandsschalten begünstigt. [110] Für LSMO $/ \mathrm{Al}_{2} \mathrm{O}_{3}$ wird in dieser Arbeit $\mathrm{RMS}=2,59 \mathrm{~nm}$ ermittelt, während für die epitaktischen LSMO/MgO-Proben der RMS $=0,76 \mathrm{~nm}$ ist. Die nanokolumnaren Schichten sind aufgrund der Defektstruktur rauer; entsprechend ist hier das Widerstandsschalten begünstigt. 


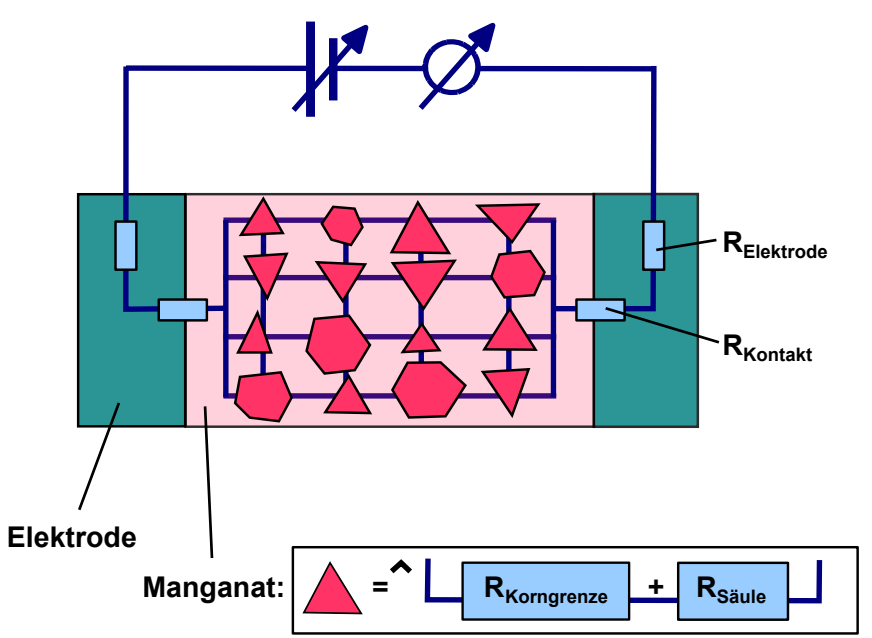

(a) Schema des Widerstandsnetzwerkes

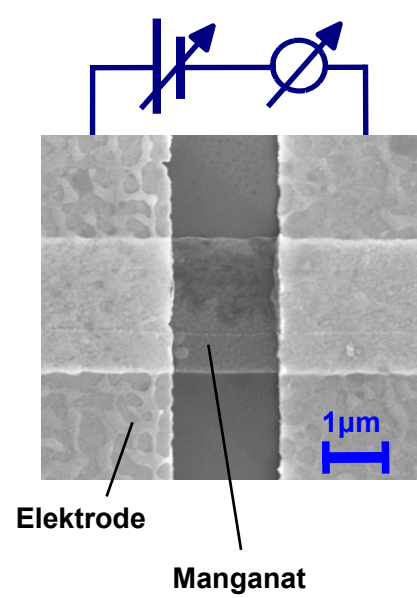

(b) REM-Aufnahme einer Mikrostruktur

Abb. 6.16: Schaltbild der Mikrostrukturmessungen. Die Manganatschicht wird durch ein Widerstandsnetzwerk aus in Reihe und parallel geschalteten Widerständen erfasst (a). Dabei setzt sich der Widerstand von einer Manganatsäule aus dem Widerstand der Korngrenze $\mathrm{R}_{\text {Korngrenze }}$ und des Korninneren $\mathrm{R}_{\text {Säule }}$ zusammen. Dieses Widerstandsnetzwerk dient zur Modellierung der mikrostrukturierten Manganatschicht (b).

In den C-AFM Messungen werden allerdings auch Schalteffekte direkt an der Schichtoberfläche beobachtet. Demnach findet wahrscheinlich ein Teil des Widerstandsschaltens auch bei den Mikrostrukturmessungen in der Oberfläche der Manganatschicht direkt unterhalb der Elektroden statt.

\section{- Elektrodenwiderstand und Zuleitungen}

Schließlich ist in dem Schaltbild des Widerstandsnetzwerkes (Abb. 6.16(a)) noch der Elektrodenwiderstand eingezeichnet. Dieser ist allerdings im Vergleich zu den anderen Widerständen vernachlässigbar klein, da als Elektrodenmaterialien $50 \ldots 100 \mathrm{~nm}$ dicke Goldschichten verwendet wurden. Auch die Zuleitungswiderstände können bei den großen Widerständen der Mikrostrukturen (teilweise einige hundert $k \Omega$ ) vernachlässigt werden.

Nachdem die unterschiedlichen Widerstände, die zu den Strom-Spannungs-Kennlinien beitragen, aufgelistet wurden, wird auf dieser Grundlage eine Simulation für das bipolare Widerstandsschalten in den Mikrostrukturen vorgestellt.

\section{Simulation der Mikrostrukturmessungen}

Im Rahmen eines Widerstandsnetzwerkes können die Kennlinien der Widerstände qualitativ gut verstanden werden. Insbesondere die Messungen der Pulsserien, die in Abb. 6.11 zu sehen sind und in denen viele verschiedene, remanente Zwischenstufen der Messwiderstände zwischen HRS und LRS beobachtet werden, können qualitativ simuliert werden, wie im Weiteren beschrieben wird.

Basis für die nachfolgende Simulation sind die Arbeiten von Chen et al. [111] und Rozenberg et al. [112]. Chen et al. simulierten zur Erklärung ihrer experimentellen Ergeb- 
nisse an $\mathrm{Pr}_{0,7} \mathrm{Ca}_{0,3} \mathrm{MnO}_{3}$ eine Reihenschaltung mit zwei Widerstandselementen. [111] Den beiden Widerstandselementen wird eine bipolare Schaltcharakteristik zugeordnet, wobei die Kennlinie der zwei Elemente spiegelsymmetrisch ist: Ein Widerstandselement schaltet bei positiven Spannungen von HRS nach LRS, während das zweite Element bei einer negativen Spannung in den tiefohmigen Zustand schaltet. Die Ergebnisse zeigen eine interessante Schaltcharakteristik, die als "table with legs" bezeichnet wird. Hierbei schaltet der Widerstand bei unterschiedlich großen Spannungen der gleichen Polarität zuerst in einen tiefohmigen Zustand und dann bei einer höheren Spannung wieder hoch in den Ausgangszustand. Bei der anschließend Verringerung der Schaltspannung bleibt der Widerstand im hochohmigen Zustand. Erst bei der entgegengesetzten Polarität wird wieder das gleiche Schaltverhalten beobachtet. Dieses Verhalten hat einige Gemeinsamkeiten mit den Ergebnissen der in Abb. 6.11 abgebildeten Messung. Auch hier wird beobachtet, dass bei einer Polarität der Gesamtwiderstand sowohl ansteigen als auch verkleinert werden kann. Ein ähnliches Verhalten wird auch in der Arbeit von Esseling beschrieben (major loop). [88]

Rozenberg et al. simulieren eine Reihenschaltung aus N Elementen $(N=100)$. [112] In jedem Spannungsschritt wird anhand der lokalen Feldstärke in einem Widerstandselement mittels der Diffusionsgleichung die lokale Sauerstoffleerstellen-Konzentration neu berechnet. Hieraus ergibt sich der lokale Widerstand und damit der Gesamtwiderstand als Funktion der angelegten Spannung. Simuliert wird ein symmetrischer Aufbau, auch hier wird eine „table with legs" Schaltcharakteristik beobachtet.

In der vorliegenden Arbeit wird eine Reihenschaltung aus $N=40$ Elementen simuliert, wie sie in Abb. 6.17(a) skizziert ist. Jedem der $N$ Widerstände $\rho_{i}$ wird eine bipolare Schaltcharakteristik zugeordnet. Diese Elemente repräsentieren die verschiedenen Manganatsäulen: Es wird dabei kein Unterschied zwischen den Elementen, die das Schalten der Korngrenzen simulieren, und denjenigen, die Schalteffekte an der Manganatoberfläche in Elektrodennähe nachbilden, gemacht.

Die Widerstandselemente werden in zwei Typen aufgeteilt: Zum einen Widerstände (Typ

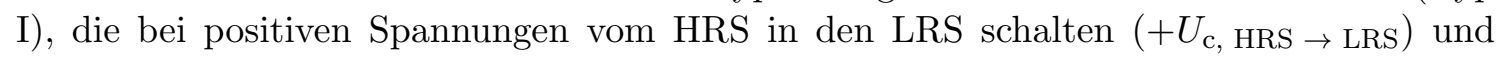
bei negativen Spannungen vom LRS in den HRS $\left(-U_{c}\right.$, LRS $\rightarrow$ HRS $)$. Der zweite Typ schal-

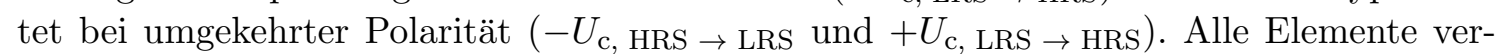
ändern beim Überschreiten der kritischen Schaltspannung den Widerstand um den konstanten Betrag $\Delta$. Folglich ergibt sich der Widerstand des LRS über $\rho_{\text {LRS }}=\rho_{\text {HRS }}-\Delta$. Die bipolaren Schaltkennlinien sind in Abb. 6.17(b) exemplarisch für je einen Widerstand vom Typ I und II dargestellt. Für das Verhältnis der kritischen Schaltspannungen wird $\left|U_{\mathrm{c}, \text { HRS } \rightarrow \text { LRS }} / U_{\mathrm{c}, \text { LRS } \rightarrow \text { HRS }}\right|=2,6$ gewählt; damit liegt das Verhältnis in der Größenordnung der Messergebnisse. ${ }^{6}$ Die Widerstände werden zufällig auf die Typen I und II verteilt. Hierfür wird die Standard C-Funktion rand() verwendet, die gleichverteilte Werte innerhalb eines vorgegebenen Wertebereiches zurückgibt (hier 0 oder 1 für Typ I und II).

Entsprechend der Ergebnisse der C-AFM und Mikrostrukturmessungen befinden sich zu Beginn der Simulation alle Widerstände im HRS. Um lokale Unterschiede zwischen den einzelnen Manganatsäulen wie Variationen der Korngrößen und Korngrenzen zu berücksichtigen, wird der Widerstand der Elemente im HRS zu Beginn der Simulation innerhalb eines fest vorgegebenen Wertebereiches variiert. Hierzu wird wiederum die rand()-Funktion verwendet.

\footnotetext{
${ }^{6}$ Die Messungen der Mikrostrukturen ergeben $\left|U_{\mathrm{c}, \mathrm{HRS}} \rightarrow \mathrm{LRS} / U_{\mathrm{c}, \text { LRS } \rightarrow \text { HRS }}\right|=1,1 \ldots 5,5$.
} 
Die Simulation berechnet den Gesamtwiderstand $R$ als Funktion der variierenden Gesamtspannung $U$ (Schaltspannung). Im ersten Simulations-Schritt wird $R$ aus den Widerständen der einzelnen Elemente $\rho_{i}$ bestimmt. Da es sich um eine Reihenschaltung handelt, gilt

$$
R=\sum_{i=1}^{40} \rho_{i} .
$$

Hieraus kann der Strom $I=U / R$ ermittelt werden, aus dem wiederum die an jedem Widerstand lokal anliegende Spannung $u_{i}$ über

$$
u_{i}=\rho_{i} \cdot I
$$

berechnet wird. Nun muss untersucht werden, welche der Widerstände $\rho_{i}$ schalten. Damit ein Widerstand schaltet, muss die vom Widerstandstyp (I oder II) und dem (aktuellen) Zustand des Elements (HRS oder LRS) abhängige, kritische Schaltspannung überschritten werden. Schaltet ein Element, so berechnet sich der neue Widerstand über

$$
\rho_{\mathrm{i}, \text { neu }}=\rho_{i} \pm \Delta \text {. }
$$

Diese Abfrage wird so oft für alle N Elemente bei einer Spannung $U$ wiederholt, bis kein Schaltprozess bei dieser Spannung mehr auftritt. ${ }^{7}$ Dann werden der Gesamtwiderstand $R$ und die zugehörige Spannung $U$ als ein Wertepaar der $\mathrm{R}(\mathrm{U})$-Kennlinie ausgegeben. Die Simulation startet für die nächste Spannung erneut bis schließlich eine komplette Kennlinie $\left(0 \rightarrow \mathrm{U}_{\max } \rightarrow-\mathrm{U}_{\max } \rightarrow 0\right)$ berechnet ist.

Um die Ergebnisse der Mikrostrukturmessungen in Abb. 6.11 zu simulieren, wurde die maximale Schaltspannung entsprechend dem Experiment variiert. Die Ergebnisse der Simulation $^{8}$ sind in Abb. 6.17 gezeigt und stimmen gut mit den Messergebnissen (Abb. 6.11) überein. Der qualitative Verlauf der Kennlinien der Messung kann für die unterschiedlichen $\mathrm{U}_{\max }$ in der Simulation wiedergegeben werden: Es können je nach maximaler Schaltspannung unterschiedliche, remanente Widerstände eingestellt werden. Zu einem abweichenden Verlauf der Kennlinie kommt es im Bereich negativer Spannungen während der dritten Hysterese (Abb. 6.17(e)) . Hier zeigt die Messung einen Widerstandsbetrag unter der Stufe $A$, während die Simulation oberhalb von $A$ bleibt. Dennoch ist die Übereinstimmung zwischen den Messergebnissen und der Simulation sehr gut, insbesondere wenn die folgenden Punkte beachtet werden.

- Ein wichtiger Aspekt ist, dass lediglich eine Reihenschaltung simuliert wird, obwohl die Mikrostrukturen eher einem zweidimensionalen Widerstandsnetzwerk entsprechen. Das bedeutet, dass ein veränderter Strompfad, der sich durch lokale Schalteffekte ergeben kann, in die Simulation nicht mit einbezogen wird, da der Stromfluss immer über die gesamte Widerstandskette erfolgt.

\footnotetext{
${ }^{7}$ Die wiederholte Abfrage bei einer Spannung wird in der Simulation implementiert, um den folgenden Fall zu berücksichtigen: Wird bei einer Spannung ein Element geschaltet, so verändert sich dadurch die lokal anliegende Spannung der anderen Elemente. An diesen kann es erst aufgrund der veränderten lokalen Spannung zu Schaltprozessen kommen, wodurch ein kaskadenartiges Durchschalten mehrer Elemente entsteht.

${ }^{8}$ In der in Abb. 6.17 gezeigten Simulation wurde $\Delta=10$ b.E. (b.E. bedeutet „beliebige Einheiten“),

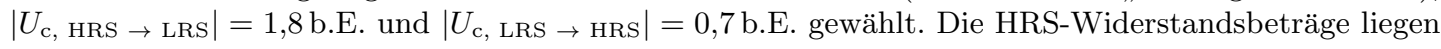
im Wertebereich von $\rho_{i}=20-29$ b.E. In der konkret gezeigten Simulation sind 23 Elemente vom Typ I (HRS $\rightarrow$ LRS bei positiven Spannungen) mit einem Gesamtwiderstand von $R_{\mathrm{I}}=556$ b.E. Für die 17 Elemente vom Typ II ist $R_{\mathrm{II}}=401 \mathrm{~b}$.E.
} 

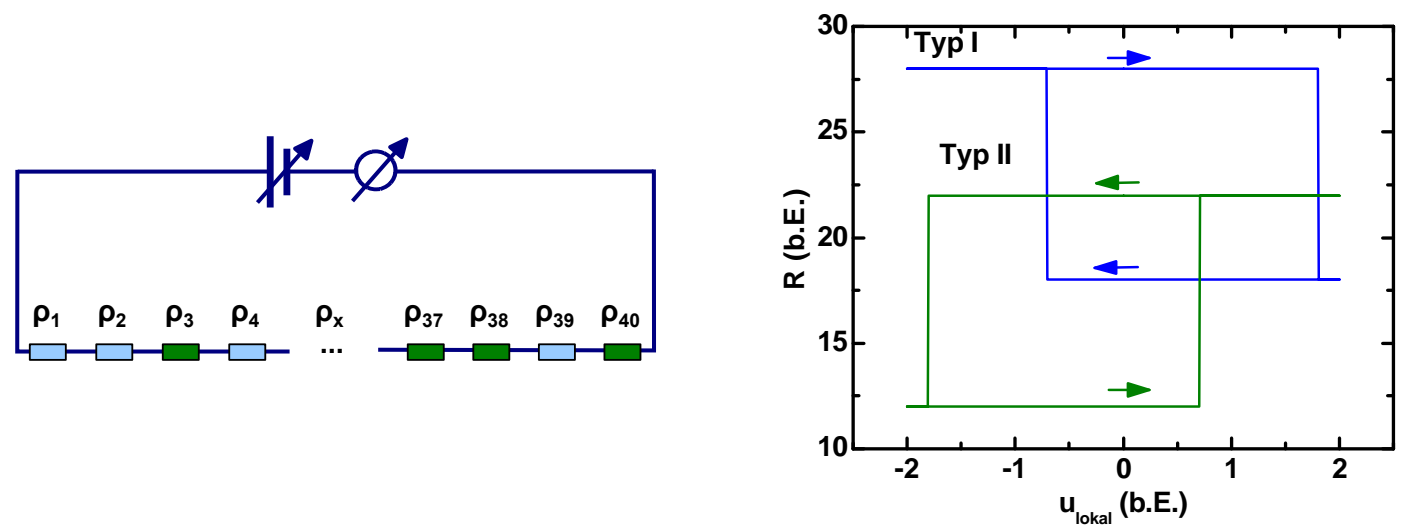

(a) Schema des simulierten Widerstandsnetzwerkes (b) Schema der Schaltcharakteristiken für Widermit zwei farblich gekennzeichneten Widerstandstypen (Typ I und II)

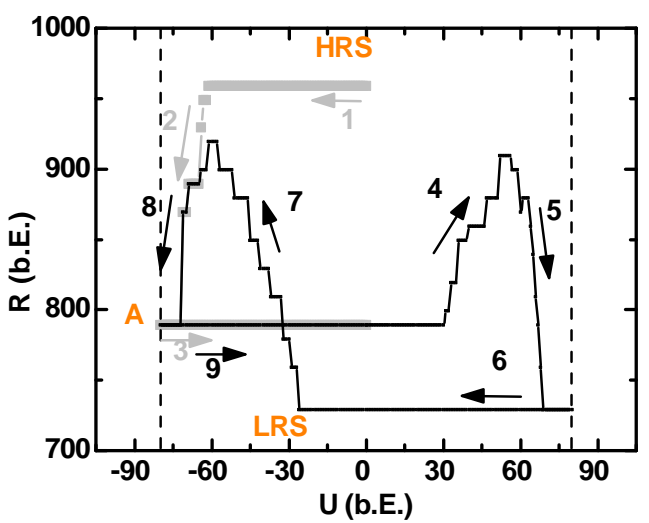

(c) 1 . Hysterese, $U_{\max }=80$ b.E.

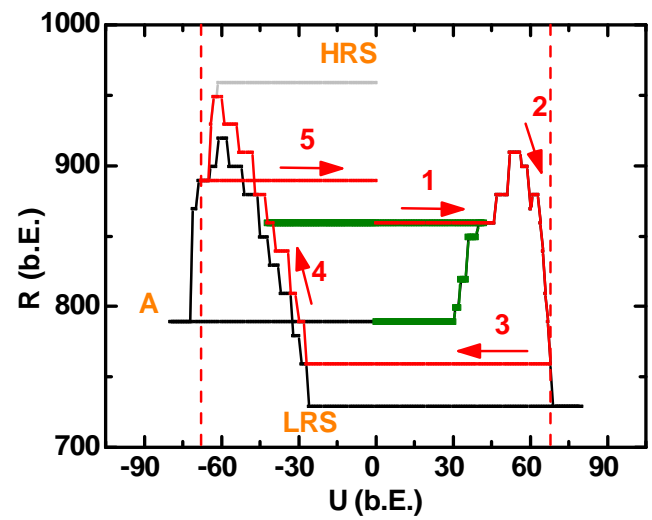

(e) 3 . Hysterese, $U_{\max }=68$ b.E.

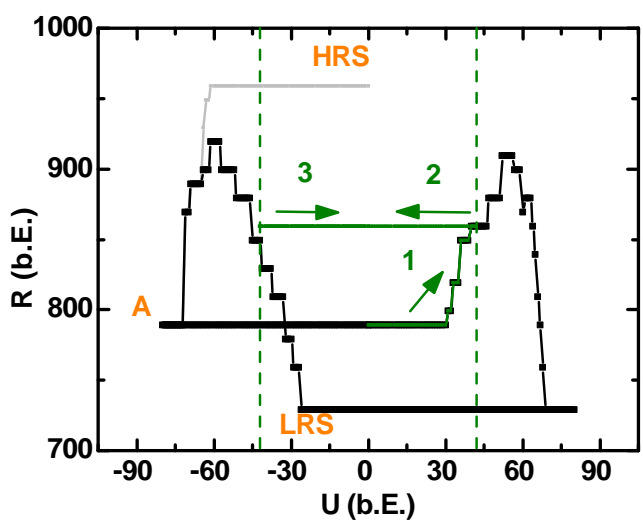

(d) 2. Hysterese, $U_{\max }=42$ b.E.

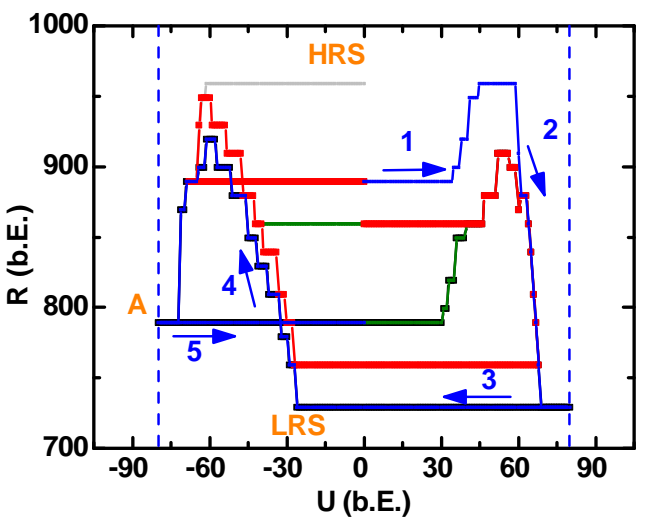

(f) 4 . Hysterese, $U_{\max }=80 \mathrm{~b} . \mathrm{E}$.

Abb. 6.17: Simulation eines Widerstandsnetzwerkes aus einer Reihenschaltung von $N=$ 40 Widerständen. Gezeigt wird eine Variation von $U_{\max }$ entsprechend der in Abb. 6.11 durchgeführten Messung. Alle Einheiten sind beliebig (b.E.). 
- Zudem wird in der Simulation für jedes Element eine bipolare Kennlinie mit einem unendlich scharfen Schaltprozess (der Schaltprozess erfolgt in einem Messschritt) angenommen. Es gibt also (für ein Element) keine Zwischenstufen zwischen HRS und LRS. Allerdings zeigen die C-AFM Messungen (Abb. 6.4), dass auch das Schalten eines einzelnen Korns über Zwischenstufen erfolgen kann.

- In der Simulation haben alle Widerstände eine gemeinsame kritische Schaltspannung. Sie unterscheiden sich aufgrund der unterschiedlichen Widerstandsbeträge. Eine Variation von $U_{C}$ für die verschiedenen Elemente führt allerdings zu ähnlichen Kennlinien wie eine Variation der Widerstände.

Ein wichtiger Bestandteil der Simulation besteht in der Annahme der zwei unterschiedlichen Elementtypen, die jeweils bei entgegengesetzten Polaritäten schalten. Diese Annahme ist zwingend notwendig, um die Schaltkennlinien zu simulieren. Aber was ist die Ursache für diese zwei verschiedenen Elementtypen?

Ein Ergebnis der C-AFM Messungen ist, dass komplette Kornhüllen der Manganatsäulen umschalten. Eine Zuordnung - etwa in die der positiven und negativen Elektrode zugewandten Bereiche der Körner - erscheint daher unwahrscheinlich. Die zwei verschiedenen Elementtypen müssen entsprechend eher in anderen lokalen Unterschieden zu finden sein. Möglicherweise spielt die Ausrichtung der Körner zwischen den Elektroden eine Rolle. Der Messaufbau von Chen et al. besteht aus einer spiegelsymmetrischen Anordnung von zwei Schaltstrukturen. [111] Hierbei ergeben sich die unterschiedlichen Typen durch die beiden spiegelsymmetrischen Elektrodengrenzflächen: In diesen ist das lokale elektrische Feld jeweils entgegengesetzt gerichtet. Die in dieser Arbeit untersuchten Mikrostrukturen sind ebenfalls symmetrisch aufgebaut. Möglicherweise wird das (entgegengesetzte) Schalten jeweils in der Nähe von einer der beiden Elektroden initiiert und schaltet in diesem Bereich die Oberflächen und Korngrenzen. Auch Scherff et al. beobachten in epitaktischen $\mathrm{Pr}_{0,7} \mathrm{Ca}_{0,3} \mathrm{MnO}_{3}$-Schichten bei der gleichen Polarität einen Anstieg und Abfall des Messwiderstands, der den zwei unterschiedlichen Manganatgrenzflächen zugeordnet werden kann. [113]

Ein Experiment zur Klärung dieses Sachverhaltes könnte aus einer kombinierten Mikrostruktur und C-AFM Messung bestehen. Wird eine Mikrostruktur, die bei einer Polarität den Widerstand vergrößert und verkleinert, während der Aufnahme der Schaltkennlinie im C-AFM untersucht, so könnte die räumliche Verteilung von HRS und LRS möglicherweise verschiedenen Domänen und Bereichen der Manganatschicht zugeordnet werden. ${ }^{9}$

\section{Widerstandsschalten und Magnetowiderstand}

Die Messungen in Abschnitt 6.2 .3 zeigen eine Kombination aus Widerstandsschalt- und Magnetowiderstandsmessungen. Hierbei können durch magnetische und elektrische Felder insgesamt vier verschiedene, remanente Widerstandswerte $\left(R_{\mathrm{HRS}}\right.$, HMS,$R_{\mathrm{HRS}}$, LMS $R_{\text {LRS, HMS }}$ und $R_{\text {LRS, LMS }}$ ) eingestellt werden. Diese Ergebnisse können gut im Rahmen des beschriebenen core shell-Modells erklärt werden, wobei sich der Widerstand einer Manganatsäule aus einem Beitrag der Kornhülle und des Korninneren zusammensetzt. Während das elektrische Widerstandsschalten im Bereich der Korngrenze stattfindet, können die Magnetowiderstandseffekte dem Korninneren der Manganatsäulen zugeordnet werden. Der Magnetowiderstand ergibt sich, wie in Abschnitt 5.5 beschrieben, durch Variationen

\footnotetext{
${ }^{9}$ Allerdings kann auch mit dieser Messung nicht die Oberfläche der Manganatschicht direkt unterhalb der makroskopischen Elektroden untersucht werden.
} 

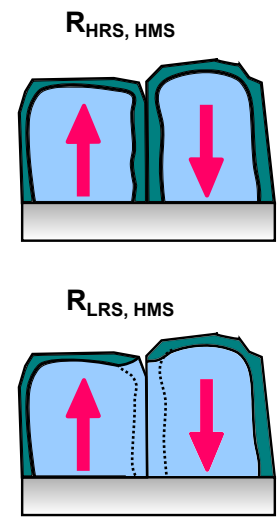

(a) Schema der verschiedenen Widerstandszustände

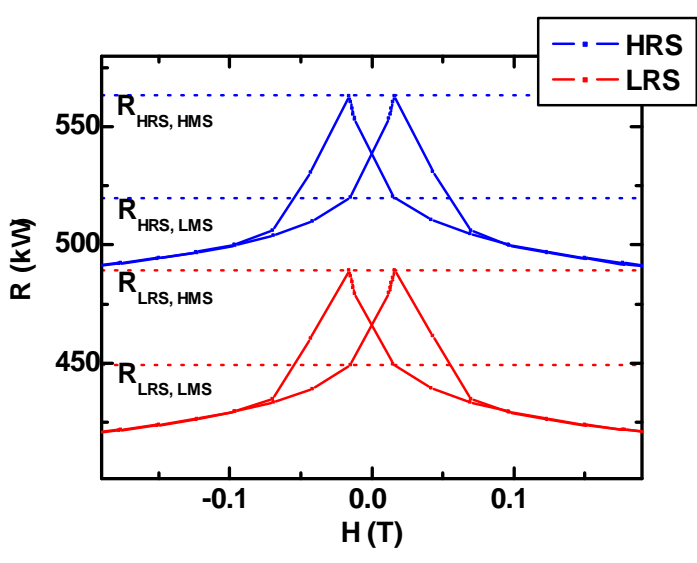

(b) Magnetowiderstand im HRS und LRS

Abb. 6.18: (a) Funktionsschema der vier verschiedenen Widerstandszustände $\left(R_{\mathrm{HRS}}\right.$ HMS $R_{\mathrm{HRS}, \mathrm{LMS}}, R_{\mathrm{LRS}}$, HMS und $R_{\mathrm{LRS}}$, LMS $)$, die durch eine Kombination von Widerstandsschalten und Magnetowiderstand eingestellt werden können. In (b) sind zum direkten Vergleich die Messergebnisse (aus Abb. 6.12) gezeigt.

der Koerzitivfelder in benachbarten Manganatsäulen. Sind dadurch die Spins benachbarter Körner parallel bzw. antiparallel ausgerichtet, ergeben sich die unterschiedlichen Widerstandswerte (HMS und LMS). Dieses Szenario ist in Abb. 6.18 schematisch zusammengefasst.

Die gezeigten Messungen wurden an mikrometergroßen Strukturen aufgenommen: Es handelt sich also um elektrische Transportmessungen an einer Vielzahl von Manganatsäulen. Daher kann keine Aussage darüber getroffen werden, ob die gleichen Körner, die das elektrische Widerstandsschalten dominieren, auch die Körner sind, die am meisten zu dem Magnetowiderstandseffekt beitragen. Für den Magnetowiderstand ist die Form der Barriere, welche die magnetischen Körner voneinander trennt, entscheidend. Sind an dem elektrischen Widerstandsschalten und an dem Tunnelmagnetowiderstand die gleichen Manganatsäulen beteiligt, müsste durch das elektrische Widerstandsschalten die Tunnelbarriere verändert werden. Dieser Einfluss scheint hier allerdings gering, es kann keine signifikante Veränderung in der Form der R(H)-Kurven im HRS und LRS beobachtet werden.

Eine Kombination von TMR mit elektrischem Widerstandsschalten wird auch in Ref. [88, 114, 115, 116] beobachtet. In diesen Arbeiten [114, 115, 116] werden magnetische Tunnelelemente mit MgO-Barrieren untersucht, wobei auf die Bedeutung einer Kombination der beiden Effekte für die gleichzeitige Datenspeicherung und als Logik-Bauelemente verwiesen wird.

\section{Einfluss der Temperatur}

In diesem Abschnitt werden die temperaturabhängigen Mikrostrukturmessungen (Messergebnisse des Abschnitts 6.2.4) diskutiert und mit den Ergebnissen anderer Forschungsgruppen verglichen. Die temperaturabhängigen Messungen zeigen, dass zwischen $10 \ldots 180 \mathrm{~K}$ keine qualitative Veränderung der Kennlinienform beobachtet wird. Die kritischen Schaltströme und Spannungen unterscheiden sich temperaturabhängig nicht mehr als bei wiederholten Messungen bei einer Temperatur $(T=10 \mathrm{~K}$, siehe Abb. 6.13). Das Gleiche gilt 
für den EPIR-Betrag $\left(\mathrm{EPIR}=\left(R_{\mathrm{HRS}}-R_{\mathrm{LRS}}\right) / R_{\mathrm{LRS}}\right)$, wie in Abb. 6.14(a) zu sehen ist. Des Weiteren wird in den temperaturabhängigen Messungen bei höheren Temperaturen eine Asymmetrie in den Kennlinien beobachtet. Im Folgenden wird zuerst die Temperaturabhängigkeit des Schalteffektes mit den Ergebnissen der Literatur verglichen, am Ende dieses Abschnittes wird auf die Asymmetrie bei höheren Temperaturen eingegangen.

In (La,Pr,Ca)-Manganat Polykristallen untersuchten Quintero et al. den Einfluss der Temperatur auf die Schaltcharakteristiken. 64] Sie finden, dass der EPIR-Effekt bei einer Temperatur $\mathrm{T}^{*}$ unterhalb der Curie-Temperatur stark erhöht ist. Auch in polykristallinen $\mathrm{Nd}_{0,7} \mathrm{Sr}_{0,3} \mathrm{MnO}_{3}$-Proben beobachten Chen et al. eine Temperaturabhängigkeit des EPIR: Der EPIR ist im Bereich des Metall-Isolator-Übergangs am größten. [117] Messungen von Liao et al. [118] zur Temperaturabhängigkeit in $\operatorname{Pr}_{0,7} \mathrm{Ca}_{0,3} \mathrm{MnO}_{3}$ dagegen zeigen, dass mit sinkender Temperatur die Hystereseeffekte in den I(V)-Kennlinien kleiner werden und für $T<140 \mathrm{~K}$ verschwinden. ${ }^{10} \mathrm{In} \mathrm{La}_{0,7} \mathrm{Ca}_{0,3} \mathrm{MnO}_{3}$ finden Shang et al. Hysteresen in den $\mathrm{I}(\mathrm{V})$ Kennlinien ausschließlich für Temperaturen oberhalb von $225 \mathrm{~K}$. [119]

Die Ergebnisse der Literatur sind entsprechend stark heterogen und weichen auch teilweise drastisch von den Ergebnissen dieser Arbeit ab. So wird im Gegensatz zu den Ergebnissen von Liao et al. und Shang et al. ein im Wesentlichen unverändertes Widerstandsschalten bis zu einer Temperatur von $2 \mathrm{~K}$ beobachtet. Möglicherweise können diese Unterschiede durch die verschiedenen Manganatsysteme und Mikrostrukturen erklärt werden. In diesem Zusammenhang wäre als weiterführende Untersuchung eine systematische Analyse der Temperaturabhängigkeit des Widerstandsschaltens in unterschiedlichen Manganatsystemen (die möglichst ähnlich präpariert werden) sehr aufschlussreich.

Welche Ergebnisse gibt es für das in dieser Arbeit untersuchte $\mathrm{La}_{1-x} \mathrm{Sr}_{x} \mathrm{MnO}_{3}$-System in der Literatur? Sawa et al. finden in $\mathrm{La}_{0,7} \mathrm{Sr}_{0,3} \mathrm{MnO}_{3}$-Schichten keine Schalteffekte, erst wenn eine Einheitszellendicke $\mathrm{Sm}_{0,7} \mathrm{Ca}_{0,3} \mathrm{MnO}_{3}$-Schicht zwischen dem $\mathrm{La}_{0,7} \mathrm{Sr}_{0,3} \mathrm{MnO}_{3}$ und der Elektrode eingebracht wird, beobachten sie Widerstandsschalten. 63] Auch Xie et al. untersuchten $\mathrm{La}_{0,67} \mathrm{Sr}_{0,33} \mathrm{MnO}_{3} / \mathrm{LaAlO}_{3}$ und finden nur in Schichten mit hoher Schichtrauigkeit Widerstandsschalten. [110] Diese Ergebnisse zeigen, dass anscheinend insbesondere in epitaktischen, glatten LSMO-Proben das Widerstandsschalten schwieriger als in anderen Manganatsystemen zu beobachten ist. ${ }^{11} \mathrm{Das} \mathrm{La}_{1-x} \mathrm{Sr}_{x} \mathrm{MnO}_{3}$-System zeigt für Manganate (bei $x \approx 0,3$ ) eine relativ große Bandbreite, die mit einer hohen Leitfähigkeit verbunden ist. Die Volumenstruktur ist die R $\overline{3}$ c-Perowskitstruktur. Diese entspricht möglicherweise schon dem LRS-Zustand, weswegen das Widerstandsschalten ungünstiger ist. Nähere Betrachtungen hierzu werden im nachfolgenden Abschnitt 6.3.4 vorgestellt.

Lau et al. berichten Widerstandsschalten in epitaktischen LSMO-Schichten bei Raumtemperatur. [120] Allerdings werden in dieser Veröffentlichung nicht viele Details über die Defektstruktur oder Oberflächenrauigkeit angegeben. Interessanterweise beobachten auch Lau et al. asymmetrische Kennlinien, obwohl ein symmetrischer Messaufbau verwendet wird. Lau et al. führen einen Elektroformierungsschritt durch, wodurch die asymmetrischen Kennlinien erklärt werden können. Auch in den Ergebnissen in Abb. 6.13(e) kommt es im Laufe der ersten Messung bei Raumtemperatur zu einem irreversiblen Schaltvorgang: Erst nach diesem sind die Kennlinien (bei hohen Temperaturen) asymmetrisch. Diese irreversible Änderung kann möglicherweise mit der Elektroformierung von Lau et al. [120]

\footnotetext{
${ }^{10}$ Diese Messergebnisse werden durch eine verringerte Sauerstoff-Diffusion bei tiefen Temperaturen erklärt.

${ }^{11}$ Mittels C-AFM Messungen wird im $\mathrm{La}_{1-x} \mathrm{Sr}_{x} \mathrm{MnO}_{3}$-System dagegen oft Widerstandsschalten berichtet. Hierbei sind die elektrischen Feldstärken und die Oberflächenbeiträge zu den Kennlinien größer. 60. 108, 109
} 
assoziiert werden. Dennoch ist das Erstaunliche an der Messung in Abb. 6.13, dass in vorherigen und anschließenden Messungen bei tiefen Temperaturen keine Veränderung des Schaltverhaltens also symmetrische Kennlinien auftreten. Der zu der Asymmetrie führende Prozess scheint unabhängig von dem Widerstandsschalten zu sein.

Was also verursacht diese Asymmetrie, die auch in weiteren Messungen bei immer tieferen Temperaturen sichtbar wird? Möglicherweise handelt es sich hier um eine Degradation, die sich durch die hohen Temperaturen und zusätzlich dem damit einhergehenden erhöhten Widerstand ergeben. Auch die Elektroden können eine wichtige Rolle spielen. Diese bestehen hier aus etwa $5 \mathrm{~nm}$ dicken Chromschichten und $50 \ldots 100 \mathrm{~nm}$ dicken Goldelektroden (Lau et al. verwenden nur Goldelektroden).

Insbesondere die asymmetrischen Kennlinien, die im Laufe der Messungen bei immer tieferen Temperaturen auftreten, können nicht abschließend verstanden werden. Dementsprechend sind weitere Messungen notwendig, um die Asymmetrie in den temperaturabhängigen Schaltkennlinien zu erklären. An dieser Stelle muss daher das beschriebene Verhalten noch unbegründet bleiben, bis weitere Versuchsreihen eine zufriedenstellende Erklärung liefern können.

\subsubsection{Mikroskopische Mechanismen des Widerstandsschaltens}

In diesem Abschnitt wird der dem Widerstandsschalten zugrunde liegende mikroskopische Mechanismus in Bezug auf die vorgestellten Messergebnisse diskutiert. Dabei wird insbesondere das Modell eines lokalen strukturellen Übergangs an den Manganatgrenzflächen der Modellvorstellung einer Diffusion und Elektromigration von Sauerstoffleerstellen gegenübergestellt. Die Grundlagen dieser Konzepte werden im Abschnitt 3.2 beschrieben. Hier wird im Folgenden zunächst auf die Bedeutung einer ausgeprägten Defektstruktur für beide Modellvorstellungen eingegangen und anschließend die Messergebnisse im Rahmen der jeweiligen Modelle diskutiert.

\section{Struktureller Übergang}

In der Arbeitsgruppe, aus welcher die vorliegende Arbeit stammt, wurde ein Modell zur Beschreibung der bipolaren Schaltcharakteristiken auf der Grundlage eines lokalen strukturellen Übergangs entwickelt.[60, 88, 93, 121] Hierbei ist insbesondere die Doktorarbeit von Krisponeit hervorzuheben, aus welcher das Funktionsschema in Abb. 3.3 für den Übergang an einer Manganatoberfläche entnommen ist. [60] Der zu Anfang vorliegende HRSZustand ist durch die Jahn-Teller verzerrten, in der Ebene der Schichtoberfläche ausgerichteten Orbitale geprägt. Durch ein (genügend hohes) elektrisches Feld (der entsprechenden Polarität) kann die Jahn-Teller-Verzerrung der $\mathrm{MnO}_{6}$-Oktaeder an der Oberfläche aufgehoben werden. Daraus resultiert ein Übergang von einer niedersymmetrischen, JahnTeller verzerrten Phase zu einer Phase höherer Symmetrie. Als Arbeitshypothese wird von Krisponeit der Übergang zwischen der Pnma- (für den HRS) und der R⿳亠丷厂c-Struktur vorgeschlagen. [60]

Vor diesem Hintergrund stellt sich die Frage, wie im Rahmen dieses Modells die in den Abschnitten 6.1 und 6.2 vorgestellten Schaltexperimente an den nanokolumnaren Manganatschichten verstanden werden können. Zur Beantwortung dieser Frage wird wiederum auf das core shell-Modell (Hülle und Kern) zurückgegriffen. [98, 100, 101, 104] Im core shell-Modell wird (bei genügend kleinen Korndurchmessern) angenommen, dass im Inneren (Kern) der Körner andere physikalische Eigenschaften als am Rand (Hülle) vorliegen. 


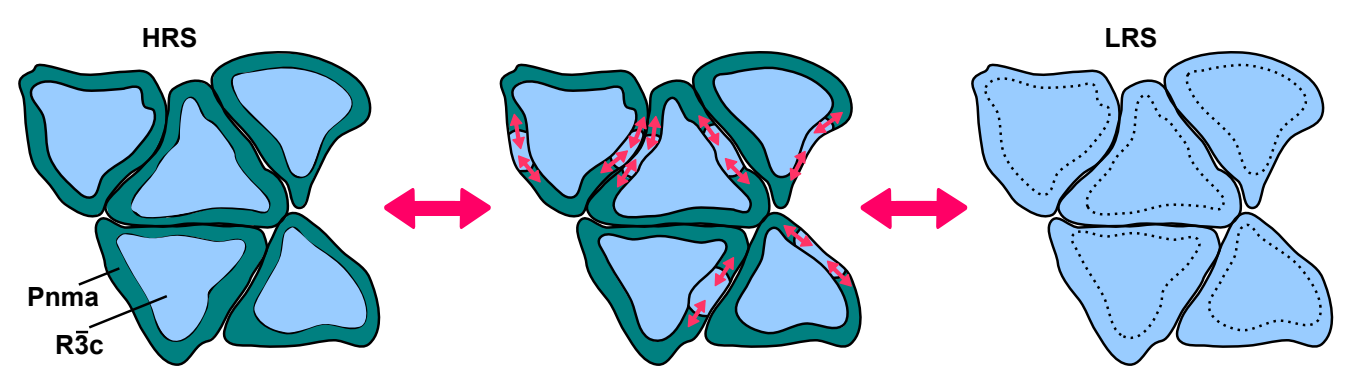

(a) Querschnitt innerhalb der Schichtebene

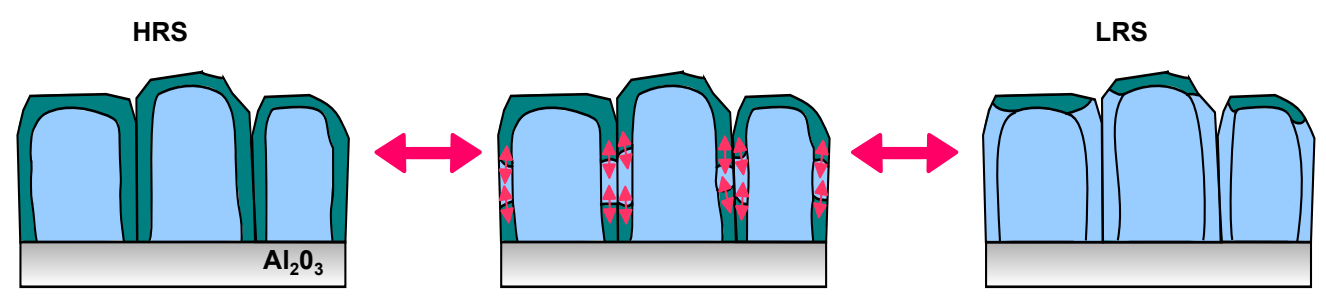

(b) Längsschnitt durch die Schicht und das Substrat

Abb. 6.19: Core shell-Modell des Widerstandsschalten der Korngrenzen im elektrischen Feld. Im HRS sind die Kornhüllen in der niedersymmetrischen Modifikation der Perowskitstruktur (beispielsweise Pnma), im LRS dagegen findet sich die die Volumenstruktur des Korninneren $(\mathrm{R} \overline{3} \mathrm{c})$ an den Kornhüllen.

Experimentell wird hierbei eine Abnahme der Dicke der Hülle mit zunehmender Korngröße beobachtet. [98, 104, 105] Sarkar et al. entdeckten in $\mathrm{La}_{0,5} \mathrm{Ca}_{0,5} \mathrm{MnO}_{3}$-Proben in Abhängigkeit der Korngröße sogar eine veränderte Kristallstruktur. [122]

Die C-AFM Messungen zeigen, dass das Widerstandsschalten bevorzugt im Bereich der Grenzflächen lokalisiert ist oder zumindest initiiert wird. Vollzieht die Hülle einen lokalen strukturellen Übergang, bedeutet das, dass in Abhängigkeit des Widerstandszustands (HRS oder LRS) die Korngrenzen und das Korninnere in unterschiedlichen Modifikationen der Perowskit-Kristallstruktur sein können. Dieser Sachverhalt wird in Abb. 6.19 verdeutlicht. Aufgrund der Symmetriebrechung an den Korngrenzen wird hier als Ausgangszustand eine niedersymmetrischere (und damit hochohmigere) Phase als im Korninneren angenommen. Der Einfluss eines elektrischen Feldes kann möglicherweise im Bereich der Korngrenzen der Nanosäulen einen strukturellen Phasenübergang bewirken, wobei die höhersymmetrische, leitfähigere Phase des Korninneren (bei LSMO wird R $\overline{3} c$ erwartet) eingestellt bzw. auf den Bereich der Korngrenzen ausgedehnt wird. Dies entspricht möglicherweise einem Übergang des Korngrenzenwiderstands von HRS nach LRS bzw. einem strukturellen Übergang zwischen Pnma und R $\overline{3} c$, wie von Krisponeit für freie Oberflächen vorgeschlagen wird. [60] Es ist allerdings zu beachten, dass kein direkter Nachweis der Kristallstrukturen vorliegt. Daher handelt es sich bei den beiden vorgeschlagenen Kristallmodifikationen auch hier lediglich um eine Arbeitshypothese.

Die Annahme eines Übergangs zwischen den genannten Kristallmodifikationen bietet sich im vorliegenden Fall aus den folgenden Gründen an: Ein Übergang von der Pnma- zu der R $\overline{3}$ c-Struktur ist auch im Phasendiagramm von $\mathrm{La}_{1-x} \mathrm{Sr}_{x} \mathrm{MnO}_{3}$ als Funktion von $x$ zu finden, wie im Abschnitt 2.4 beschrieben wird. Dieser strukturelle Phasenübergang wird zusätzlich als Funktion der Sauerstoffleerstellen-Konzentration beobachtet. [29] Im 
Rahmen des oben beschriebenen core shell-Modells erscheint es daher sinnvoll, im Korninneren die metallische R $\overline{3}$ c-Volumen-Phase anzunehmen. Für die Schale (shell) wird ein isolierender und niedersymmetrischer Ordnungszustand erwartet, der möglicherweise mit der Pnma-Struktur assoziiert werden kann.

Darüber hinaus wird ein Phasenübergang zwischen Pnma und $R \overline{3} c$ beispielsweise auch in Kompositproben beobachtet. Zahlreiche Untersuchungen widmen sich diesen Systemen, in denen ein Manganat mit einem anderen Material kombiniert und durch dieses gezielt beeinflusst werden kann. So wird etwa in granularen $\left(\mathrm{La}_{0,7} \mathrm{Ca}_{0,3} \mathrm{MnO}_{3}\right)_{1-x}(\mathrm{MgO})_{x} \mathrm{Kom}-$ positproben ein struktureller Übergang in Abhängigkeit von der MgO-Konzentration gefunden. [123, 124] Die Kristallstruktur des Manganats verändert sich hierbei von Pnma zu $\mathrm{R} \overline{3} \mathrm{c}$.

Zu dem speziellen Fall von strukturellen Phasenübergängen an Korngrenzen gibt es von Frolov et al. eine relativ neue, theoretische Studie. [125] Diese bezieht sich allerdings auf Weitwinkelkorngrenzen in metallischen Systemen. Hierbei werden multiple, metastabile Zustände in den Weitwinkelkorngrenzen beobachtet, die als Funktion der Temperatur und Punktdefekte-Konzentration in den Korngrenzen reversible Übergänge erster Ordnung zwischen verschiedenen Korngrenzenphasen zeigen.

In den C-AFM Messungen an der $300 \mathrm{~nm}$ dicken Schicht wird schon im Ausgangszustand ein signifkanter Anteil von LRS beobachtet (siehe Abb. 6.7). Experimentelle Untersuchungen im Rahmen des core shell-Modells zur Abhängigkeit der Kornhüllendicke $t$ von der Korngröße $d$ zeigen eine Abnahme von $t$ für größere Körner. [98, 104] Balcells et al. [98] etwa finden ab einem Korndurchmesser von $300 \mathrm{~nm}$ keinen messbaren Beitrag einer Kornhülle mehr. ${ }^{12}$ Zhu et al. beobachten schon für Korndurchmesser von $d=50 \mathrm{~nm}$ keinen Hüllenbeitrag mehr. [104] Auch hier handelt es sich um Untersuchungen an polykristallinen $\mathrm{La}_{2 / 3} \mathrm{Sr}_{1 / 3} \mathrm{MnO}_{3}$-Proben. Zhu et al. charakterisieren das Korninnere als metallisch und die Schale als isolierendes, ungeordnetes Spin-Glass. Die erhöhte Leitfähigkeit in den Messungen der Abb. 6.7 kann also vermutlich dadurch erklärt werden, dass für die dicke Schicht schon zu Beginn teilweise die R⿳亠丷厂c-Phase des Korninneren an der Kornhülle vorhanden ist.

\section{Sauerstoffleerstellen-Diffusion}

In der Literatur wird zur Erklärung des bipolaren Schalteffekts oft die SauerstoffleerstellenDiffusion als zugrunde liegender mikroskopischer Mechanismus angesehen. Für den Bereich der Manganate sind hier insbesondere die Veröffentlichungen von Baikalov et al.[15] und Rozenberg et al.[112] zu nennen. Die Modellvorstellung beruht dabei darauf, dass das elektrische Feld lokal die Sauerstoffleerstellenkonzentration verändert. Diese hat insbesondere bei den Manganaten einen starken Einfluss auf den Widerstand. [29] Welche Rolle spielt hierbei die lokale Defektstruktur? Korngrenzen und Oberflächen sind auch für die Sauerstoffleerstellen-Diffusion wichtig, da hier die Diffusionskonstanten drastisch erhöht sein können. Auf diesen Zusammenhang machen insbesondere Szot et al. auf der Grundlage ihrer Untersuchungen an $\mathrm{SrTiO}_{3}$ aufmerksam, wobei Versetzungskerne als Kanäle für schnelle Diffusion angesehen werden. [19]

Theoretische Rechnungen zeigen, dass der Diffusionskoeffizient in Korngrenzen mehrere Größenordnungen größer als im Volumen der Probe sein kann. [126] Untersuchungen an

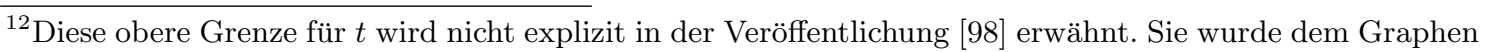
$t(d)$ in Abb. 2(b) entnommen. 
Manganaten hierzu werden von De Souza et al. und Berenov et al. vorgestellt. [127, 128] De Souza et al. berichten in $\mathrm{La}_{0,8} \mathrm{Sr}_{0,2} \mathrm{MnO}_{3+\delta}$-Proben, dass die Diffusionskonstante der Korngrenzen $D_{\mathrm{KG}}$ um einen Faktor von $10^{5}$ höher als im Volumen der Probe ist $(T=$ $700 \ldots 1000^{\circ} \mathrm{C}$ und bei Annahme einer Korngrenzenbreite im nm-Bereich). [127] Berenov et al. [128] finden in $\mathrm{LaMnO}_{3 \pm \delta}$ und $\mathrm{La}_{0,9} \mathrm{MnO}_{3 \pm \delta}$ um drei Größenordnungen unterschiedliche Diffusionskonstanten. ${ }^{13}$ Die Diffusionskonstanten sind entsprechend stark von der Mikrostruktur abhängig, wobei insbesondere die Korngrenzen für die Diffusion der Sauerstoffvakanzen eine wichtige Rolle spielen.

Allerdings müssen das Modell einer Sauerstoffleerstellen-Diffusion und das eines strukturellen Übergangs einander nicht zwingend ausschließen. Liao et al. beobachten in TEM Messungen im elektrischen Feld in $\operatorname{Pr}_{0.7} \mathrm{Ca}_{0.3} \mathrm{MnO}_{3}$-Proben strukturelle Streifenphasen, die sich in Abhängigkeit des elektrischen Feldes ausbilden. [118] Diese Strukturen werden mit einer lokalen Ordnung der Sauerstoffleerstellen in Verbindung gebracht, deren Bewegung durch das elektrische Feld induziert wird. Zudem wird von Mitchell et al. in

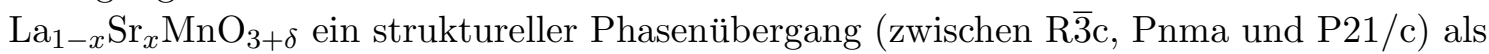
Funktion der Sauerstoffleerstellenkonzentration und Dotierung beobachtet. [29]

\section{Einordnung der Ergebnisse in die vorgestellten mikroskopischen Mechanismen}

Nachdem der Einfluss von Defektstrukturen sowohl auf die Sauerstoffleerstellen-Diffusion als auch auf einen lokalen strukturellen Übergang erläutert wurde, werden im Folgenden die Messergebnisse anhand dieser Modelle diskutiert.

\section{- Einfluss der Temperatur}

Die Temperaturabhängigkeit des Widerstandsschaltens wird im Abschnitt 6.2.4 vorgestellt. Es wird Widerstandsschalten bei Temperaturen von nur $2 \mathrm{~K}$ beobachtet. Zusätzlich finden sich in einem Temperaturbereich zwischen $10 \ldots 180 \mathrm{~K}$ keine qualitativ veränderten Kennlinien und Schaltparameter. Diffusionsprozesse sind aber thermisch aktiviert, für die Diffusionskoeffizienten gilt $D \propto e^{-E /\left(k_{B} T\right)}$. Hierbei ist $E$ die Aktivierungsenergie $(E=0,4 \mathrm{eV}[118,129])$. Damit ergibt sich für das Verhältnis bei verschiedenen Temperaturen

$$
\frac{D(10 \mathrm{~K})}{D(180 \mathrm{~K})} \approx e^{-439}
$$

Entsprechend dieser drastisch unterschiedlichen Diffusionskonstanten ist ein großer Unterschied zwischen den Messungen bei $10 \mathrm{~K}$ und $180 \mathrm{~K}$ zu erwarten. Dieses stimmt allerdings nicht mit den Beobachtungen der vorliegenden Arbeit überein.

\section{- Geometrie der LRS-Bereiche}

Die C-AFM Messungen zeigen, dass eine hohe Schaltspannung komplette Randbereiche von Körnern in den LRS schalten kann (siehe beispielsweise die Dreiecke in Abb. 6.1). Es erscheint unmöglich, dass entlang des kompletten Randbereiches das elektrische Feld bzw. der Strompfad so ausgerichtet ist, dass Diffusionsprozesse zu einem Schalten etwa von HRS nach LRS führen können.

Vielmehr kann die Beobachtung dadurch erklärt werden, dass ein struktureller Übergang, der mit einer elastischen Verzerrung des geschalteten Bereiches in der umgebenden Matrix verbunden ist, aufgrund der elastischen Verzerrungsenergie den kompletten Randbereich umschalten kann. Um dieses Ergebnis im Rahmen der Sauerstoffleerstellen-Diffusion zu verstehen, muss eine Erklärung für eine durch das elastische

\footnotetext{
${ }^{13}$ Bei der Berechnung wird eine Korngrenzenbreite von $1 \mathrm{~nm}$ angenommen; damit ergibt sich $D_{\mathrm{KG}} \approx$
} $10^{-10} \mathrm{~cm}^{2} / \mathrm{s}\left(T=900^{\circ} \mathrm{C}\right)$. 
Feld getriebene Umschaltung des kompletten Randbereiches gefunden werden, wie etwa bei einem mit der Diffusion verbundenen strukturellen Übergang.

\section{- Kritische elektrische Feldstärken der Oberflächen und Korngrenzen}

Wie in Abschnitt 6.3.1 gezeigt wird, kann aus den C-AFM Messungen das Verhältnis der kritischen elektrischen Feldstärken an der Oberfläche und an den Korngrenzen einer nanokolumnaren Schicht grob abgeschätzt werden. Nach Gleichung 6.7 gilt

hierfür $\frac{E_{\text {Oberfläche }}}{E_{\text {Korngrenze }}} \approx 12,5$. Dieses Verhältnis ist ein weiteres Argument gegen eine Sauerstoffleerstellen-Diffusion, da im Allgemeinen die Aktivierungsenergien für eine Oberflächendiffusion geringer als bei Korngrenzen und im Volumen sind. [130]

Aber auch im Rahmen eines lokalen strukturellen Übergangs ist es erstaunlich, dass an der freien Oberfläche einer Manganatschicht die kritische elektrische Feldstärke größer als in einer Korngrenze ist. Eine mögliche Erklärung hierfür sind „tote“ Lagen (dead layers) an den Schichtoberflächen, die für Manganatschichten berichtet werden. [131] Diese sorgen möglicherweise für die an der Oberfläche erhöhten kritischen Werte, können aber auch einen Einfluss auf die Aktivierungsenergien zeigen.

\section{- Unterschiedliche kritische Schaltspannungen}

Ein Ergebnis der Mikrostrukturmessungen ist, dass das Verhältnis der für die Schaltprozesse notwendigen kritischen Spannungen für den Schaltvorgang von HRS nach LRS um einen Faktor $U_{\mathrm{c}}^{*}=1,1 \ldots 5,5$ größer ist als für den umgekehrten Fall (LRS nach HRS). Im Rahmen eines lokalen strukturellen Übergangs entspricht der an den Grenzflächen (der dünnen Schichten) energetisch günstigere HRS der niedersymmetrischen, Jahn-Teller verzerrten Struktur (Pnma). Dieses kann anhand der Symmetriebrechung und lokaler Spannungszustände an den Grenzflächen erklärt werden.

\section{- Wachstum der LRS-Bereiche}

In den Abb. 6.4 und 6.5 ist zu sehen, dass die LRS-Bereiche sich sukzessive entlang der Korngrenzen ausdehnen. Dieses kann in beiden Modellen folgendermaßen erörtert werden: Aufgrund von lokalen Inhomogenitäten in den Korngrenzen gibt es einzelne Stellen, in denen das lokale elektrische Feld höher ist und diese Bereiche entsprechend früher schalten (dieses gilt für beide Modellvorstellungen). Darüber hinaus ist es vorstellbar, dass in der Nähe von Defekten aufgrund der lokalen Spannung schon eine modifizierte Struktur vorliegt, die sich durch die Schaltspannung ausdehnen kann.

\section{- Erhöhte Leitfähigkeit im Ausgangszustand der 300 nm dicken Schicht}

C-AFM Messungen (Abb. 6.7) zeigen, dass in einer $300 \mathrm{~nm}$ dicken, polykristallinen LSMO-Schicht schon im Ausgangszustand ein signifikanter Anteil der Schicht im LRS ist. Im Rahmen einer Sauerstoffleerstellen-Diffusion kann dieses Ergebnis damit erklärt werden, dass für eine dicke Schicht die Präparationsbedingungen modifiziert sind: Da alle Depositionsparameter wie auch die Wachstumsgeschwindigkeit unverändert gehalten werden, dauert es entsprechend länger, eine dicke Schicht zu wachsen. Möglicherweise resultiert daraus eine veränderte Sauerstoffleerstellkonzentration im Bereich der Grenzflächen.

Wie bereits beschrieben, liegt eine alternative Erklärung hierfür in der Abhängigkeit der Hüllendicke von dem Korndurchmesser. [98, 104, 105]

Diese Diskussion zeigt die Schwierigkeiten bei der Unterscheidung zwischen den beiden Modellvorstellungen. Allerdings sprechen insbesondere die Temperaturabhängigkeit und die Geometrieargumente für einen lokalen strukturellen Phasenübergang und gegen eine 
Sauerstoffleerstellen-Diffusion. Um das Fundament des Modells eines lokalen strukturellen Übergangs zu prüfen und gegebenfalls zu stärken, sind Messmethoden, die eine lokale Strukturanalyse ermöglichen, von entscheidender Bedeutung. Hierzu gehören TEM und TERS (tip enhanced raman spectroscopy), allerdings ist auch bei diesen Methoden zu beachten, dass ein struktureller Phasenübergang mit einer Sauerstoffleerstellen-Diffusion verbunden sein kann.

\subsection{Zusammenfassung}

In diesem Kapitel wird das bipolare Widerstandsschalten in nanokolumnaren Manganatschichten analysiert. Aus den Messergebnissen werden qualitative und quantitative Aussagen zu den Energieniveaus und Barrieren zwischen HRS und LRS gewonnen. Eine Beschreibung gelingt im Rahmen eines Doppelmuldenpotentials, wobei zwischen einer Manganatoberfläche und Korngrenze unterschieden wird - für die Korngrenzen findet eine weitere Unterteilung als Funktion der Schichtdicke statt. Die Messergebnisse zeigen, dass der HRS an der Manganatoberfläche und an der Grenzfläche der dünnen Schichten (40..60 nm) energetisch günstiger als der LRS ist. Für die dickere Schicht $(300 \mathrm{~nm})$ hingegen ist der LRS energetisch wesentlich günstiger als für die dünne Schicht, möglicherweise liegt hier das Energieniveau des LRS sogar unter dem des HRS.

Weiterhin finden die Mikrostrukturmessungen der 40..60 nm dicken Schichten, dass die Energiebarriere, die für den Schaltvorgang von HRS nach LRS überwunden werden muss $\left(\Delta_{1}+\Delta_{2}\right)$, um einen Faktor von $1,1 \ldots 5,5\left(\hat{\wedge} \frac{\Delta_{1}+\Delta_{2}}{\Delta_{1}}\right)$ größer ist als für das Schalten von LRS nach HRS $\left(\Delta_{1}\right)$. Eine grobe Abschätzung der elektrischen Feldstärken aus den C-AFM Messungen zeigt, dass an der Oberfläche (einer dünnen nanokolumnaren Schicht) die Energiebarriere um einen Faktor von $12,5\left(\stackrel{\left(\Delta_{1}+\Delta_{2}\right)_{\text {Oberfläche }}}{\left(\Delta_{1}+\Delta_{2}\right)_{\text {Korngrenze }}}\right)$ größer ist als im Bereich der Korngrenzen.

Das Schaltverhalten der Schichten wird sowohl auf der lokalen als auch auf der globalen Ebene mittels C-AFM und Mikrostrukturmessungen analysiert. Die lokalen C-AFM Messungen zeigen die Bedeutung der Korngrenzen. Die Mikrostrukturmessungen können auf dieser Grundlage als Widerstandsnetzwerke aus Manganatsäulen interpretiert und simuliert werden. In einer Reihenschaltung aus Widerstandselementen, die jeweils eine bipolare Schaltcharakteristik haben, können die Kennlinien auch mit der Vielzahl an remanenten Zwischenzuständen modelliert werden. Zur Erklärung der Messergebnisse sind zwei verschiedene Elementtypen, die bezüglich der kritischen Schaltspannungen spiegelsymmetrisch sind (also bei einer unterschiedlichen Polarität von HRS nach LRS schalten), notwendig. Eine offene Frage bleibt die Zuordnung der verschiedenen Elementtypen beispielsweise zu verschiedenen Domänen aus mehreren Körnern oder aber zu den Bereichen in Elektrodennähe. Zur Beantwortung dieser Frage bietet sich eine (nicht triviale) Kombination der gezeigten Messmethoden an: Eine gleichzeitige Analyse der Verteilung von HRS und LRS mittels C-AFM, während die Mikrostruktur über ihre (makroskopischen) Elektroden geschaltet wird.

Die vielen remanenten Zwischenstufen sind möglicherweise in Hinblick auf nichtflüchtige Speichermedien interessant. Sind die Zwischenstufen der Widerstände weit genug voneinander entfernt, so kann ein Multibit-Speicherelement in Betracht gezogen werden. Andernfalls ist auch ein quasi analoger Speicher denkbar. Die Mikrostrukturmessungen zeigen weiterhin die Möglichkeit der Kombination aus Widerstandsschalten und Kleinfeldmagnetowiderstandsmessungen (allerdings bei $T=10 \mathrm{~K}$ ). Durch die gezielte Kombination von 
elektrischen und magnetischen Feldern ergeben sich vier verschiedene, remanente Widerstandszustände. Dieser faszinierende Zusammenhang kann möglicherweise in technischer Hinsicht von Interesse sein, beispielsweise als Sensor mit Speicherfunktion für magnetische und elektrische Felder.

Auf Grundlage der gewonnenen Messergebnisse erfolgt eine Analyse mit Blick auf die zugrunde liegenden mikroskopischen Mechanismen, hierbei wird insbesondere der Einfluss von Korngrenzen beleuchtet. Dabei wird auf das im vorherigen Kapitel eingeführte core shell-Modell zurückgegriffen. Im Rahmen des lokalen strukturellen Übergangs wird der HRS als niedersymmetrische, isolierende Hülle des Manganatkristalls angenommen, die durch ein elektrisches Feld in die metallische, höhersymmetrische Phase des Korninnern geschaltet werden kann. In der Modellvorstellung einer Sauerstoffleerstellen-Diffusion sorgen die Korngrenzen für erhöhte Diffusionskonstanten. Allerdings sind die temperaturabhängigen Messungen und die Geometrie der C-AFM Messungen nur schwer im Rahmen einer Sauerstoffleerstellen-Diffusion zu verstehen und sprechen mehr für einen lokalen strukturellen Übergang. Dennoch müssen weiterhin ortsaufgelöste Strukturmessungen dazu beitragen, ein grundlegendes Verständnis für diesen komplexen Mechanismus zu erhalten. 


\section{Photoinduzierte Änderung der ersten und dritten harmonischen Spannung}

Dieses Kapitel widmet sich der Untersuchung des Einflusses von Laserlicht auf die erste und dritte harmonische Spannung im Bereich des Metall-Isolator-Übergangs einer Manganatprobe. Die Messung von dritten harmonischen Spannungen ist für die Ermittlung einer Vielzahl von physikalischen Parametern relevant, insbesondere kann sie zur Bestimmung von thermischen Materialeigenschaften wie der spezifischen Wärme und der thermischen Leitfähigkeit herangezogen werden (beispielsweise bei filamentartigen Proben [132]). Auch bei der Erforschung der Hoch- $T_{\mathrm{c}}$-Supraleiter kommen dritte harmonische Spannungsmessungen zum Einsatz. Es wird - unter anderem - die Abhängigkeit der Sprungtemperatur vom äußeren Druck $T_{\mathrm{c}}(\mathrm{P})$ [133] und der thermische Grenzflächenwiderstand zwischen einer supraleitenden Schicht und dem Substrat bestimmt [134, 135]. Der Einfluss von Joulescher Wärme auf die Generierung von höheren harmonischen, elektrischen Signalen kann auch zur Analyse von Defekten bzw. Einkerbungen in metallischen Leiterbahnen verwendet werden. [136]

Bei einigen Manganatsystemen finden Moshnyaga et al. im Bereich der Metall-IsolatorÜbergangstemperatur eine vergrößerte, dritte harmonische Spannung, die mit dem Auftreten einer erhöhten Anzahl von korrelierten Polaronen am Übergang erklärt wird. [22] Das elektrische Feld koppelt dabei an die korrelierten Polaronen als elektrisch-elastische Quadrupole an. Diese Veröffentlichung [22] ist die Grundlage für die folgenden Untersuchungen, in denen der Einfluss von Laserlicht auf die dritte harmonische Spannung bzw. die Konzentration der korrelierten Polaronen analysiert wird.

Bei der untersuchten Probe handelt es sich um eine $\mathrm{La}_{1-x} \mathrm{Ba}_{x} \mathrm{MnO}_{3} / \mathrm{SrTiO}_{3}(100)$-Schicht $(x=0,2 \ldots 0,3)(\mathrm{LBMO})$, die von C. Ballani im Rahmen seiner Master-Arbeit präpariert wurde (Probe: X105). Die Gitterkonstante beträgt $c=3,913 \pm 0,001 \AA$ und die Schicht ist $30 \mathrm{~nm}$ dick. Die Probe wurde, wie in Abb. 4.4(a) gezeigt, mikrostrukturiert. Die Stegbreite beträgt $30 \mu \mathrm{m}$ und die Steglänge ist $130 \mu \mathrm{m}$. Es wird angemerkt, dass es sich bei den gezeigten Ergebnissen um Messungen an lediglich einer Mikrostruktur der beschriebenen Manganatprobe handelt; weiterführende Messungen sind daher erforderlich.

Das nachfolgende Kapitel gliedert sich wie folgt: Zunächst werden temperaturabhängige PPMS-Messungen der ersten und dritten harmonischen Spannung (ohne Lichteinstrah- 
lung) gezeigt, um eine allgemeine Charakterisierung und Einordnung der untersuchten Schicht und Mikrostruktur zu geben. Im Anschluss werden photoinduzierte Messungen vorgestellt, die in dem im Abschnitt 4.8 beschriebenen Aufbau gemessen wurden. Hierbei wird die Temperatur- und Zeitabhängigkeit des photoinduzierten elektronischen Transportverhaltens vorgestellt und charakterisiert. In der nachfolgenden Diskussion werden die Messergebnisse insbesondere mit der Veröffentlichung von Moshnyaga et al. [22] verglichen. Den Schluss dieses Kapitels bildet eine kurze Zusammenfassung.

Die photoinduzierten Messungen wurden in Zusammenarbeit mit der Arbeitsgruppe von Prof. Dr. M. Münzenberg, wobei insbesondere M. Walter und Dr. J. Walowski hervorzuheben sind, und mit M. Mchalwat durchgeführt.

\subsection{PPMS-Messungen der LBMO-Schicht}

Die folgenden Messungen wurden im PPMS (siehe Abschnitt 4.5) mittels der AC-Option aufgenommen. Dabei wurde $R_{\omega, 3 \omega}$ als Funktion der Temperatur (ohne Laserlicht) für die unstrukturierte und strukturierte LBMO-Probe gemessen. In Abb.7.1 ist die Messung von $U_{\omega, 3 \omega}(T)$ und $K(T)=\log \frac{U_{3 \omega}(T)}{U_{\omega}(T)}$ für die Mikrostruktur gezeigt $(I=20 \mu \mathrm{A}, f=17 \mathrm{~Hz})$. Aus dieser Messung ergibt sich für die Metall-Isolator-Übergangstemperatur $T_{\mathrm{MI}, \omega}=292 \pm$ $2 \mathrm{~K}$ und $T_{\mathrm{MI}, 3 \omega}=286 \pm 2 \mathrm{~K}$, wobei $T_{\mathrm{MI}}$ als Maximum von $U_{\omega, 3 \omega}(T)$ definiert ist. Der harmonische Koeffizient für die Mikrostruktur ist $K\left(T_{\mathrm{MI}}\right)=-34 \mathrm{~dB}$.

Für die unstrukturierte Schicht ist die Temperaturabhängigkeit von $R_{3 \omega} / \mathrm{R}_{\omega}$ in Abb. 7.2 dargestellt. Es wird $T_{\mathrm{MI}, \omega}=291 \pm 2 \mathrm{~K}$ und $T_{\mathrm{MI}, 3 \omega}=287 \pm 2 \mathrm{~K}$ ermittelt. Demnach zeigt sich kein Unterschied in $T_{\mathrm{MI}}$ zwischen strukturierter und unstrukturierter Probe. Für den harmonischen Koeffizienten der unstrukturierten Schicht gilt $K\left(T_{\mathrm{MI}}\right)=-67 \mathrm{~dB}(I=100 \mu \mathrm{A}$ und $f=17 \mathrm{~Hz}) . K$ ist also für die Mikrostruktur deutlich größer als für die unstrukturierte Schicht.

\subsection{Einfluss von Laserlicht}

In diesem Abschnitt wird der Einfluss von Laseranregungen auf die erste und dritte harmonische Spannung untersucht. Hierfür wird der im Unterkapitel 4.8 vorgestellte Messaufbau

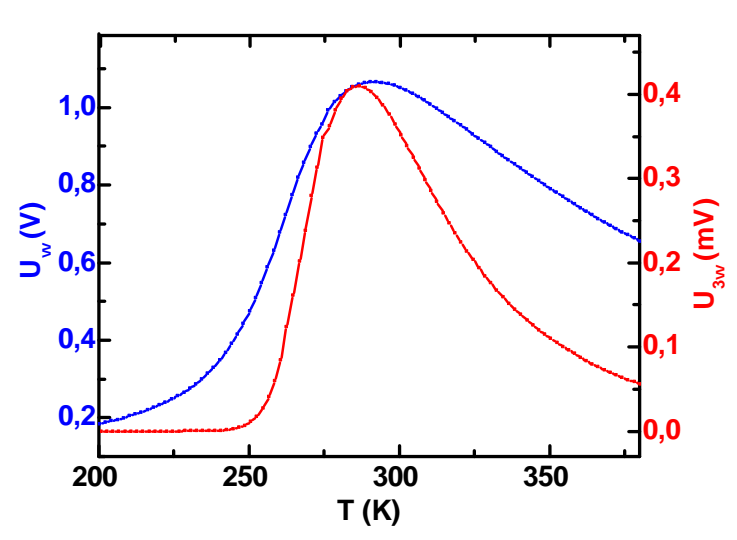

(a) $U_{\omega, 3 \omega}(T)$

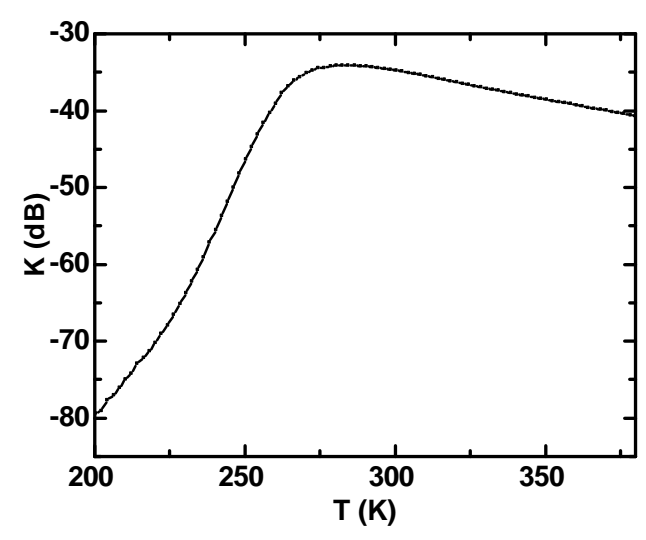

(b) $K(T)$

Abb. 7.1: PPMS-Messung: Temperaturabhängigkeit von $U_{\omega, 3 \omega}$ und $K$ (Mikrostruktur, $I=$ $20 \mu \mathrm{A}, f=17 \mathrm{~Hz})$. 


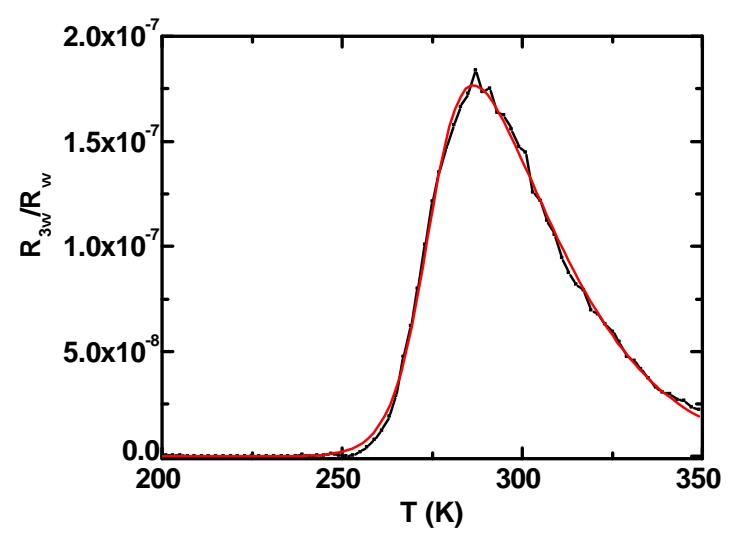

Abb. 7.2: PPMS-Messung: $R_{3 \omega} / R_{\omega}(T)$ mit Fit durch eine asymmetrische doppelte Sigmoidalfunktion, die Fitparameter werden im Abschnitt 7.3 angegeben (Messung an unstrukturierter Schicht, $I=100 \mu \mathrm{A}, f=17 \mathrm{~Hz}$ ).

verwendet. In diesem Aufbau werden $U_{\omega}, U_{3 \omega}, \theta_{\omega}$ und $\theta_{3 \omega}$ als Funktion der Zeit durch die Lock-In-Verstärker gemessen und aufgezeichnet. Am Anfang der Messungen ist der Laserstrahl immer geblockt. Nach einer fest vorgegebenen Zeit wird das den Laserstrahl blockierende Objekt aus dem Strahlengang genommen und die Probe belichtet. Anschließend wird der Laserstrahl wieder geblockt. Dieser Vorgang wird mehrere Male wiederholt. Zusätzliche Variationsparameter sind die Temperatur, die unterschiedlichen Laser (siehe Tabelle 4.8), die Laserleistung, die Frequenz der AC-Anregung, der AC-Stromfluss und die Zeitkonstanten der Lock-In-Verstärker.

\subsubsection{Temperaturabhängigkeit}

Beispiele der $U_{\omega, 3 \omega}(t)$-Kurven sind in Abb. 7.3 und 7.4 (jeweils in (a) und (b)) zu sehen. In Abb. 7.3 wird die Anregung durch den Femtosekundenlaser und in Abb. 7.4 durch den Diodenlaser vorgestellt. Die grau hinterlegten Bereiche entsprechen den Zeiten, in denen der Laserstrahl auf die Probe scheint. Die verschiedenen Kurven wurden bei unterschiedlichen Temperaturen aufgenommen. In den gezeigten Messungen ist der Strom $I=0,9 \mu \mathrm{A}$ und der Widerstand wird über $R_{\omega}=\frac{U_{\omega}}{I}$ berechnet (die Frequenz ist mit $f=17 \mathrm{~Hz}$ klein genug, um induktive und kapazitive Anteile zu vernachlässigen).

Als erstes werden die Ergebnisse bei der Anregung durch den Femtosekundenlaser beschrieben. In Abb. 7.3 (a) und (b) ist zu sehen, dass bei $T=284 \mathrm{~K}$ (schwarze Kurve) $U_{\omega}$ und $U_{3 \omega}$ kleiner werden, wenn der Laser auf die Probe scheint. Bei $T=275 \mathrm{~K}$ (orange Kurve) dagegen vergrößern sich $U_{\omega, 3 \omega}$ bzw. der Widerstand $R_{\omega, 3 \omega}$ unter dem Einfluss des Lasers. Die grüne Kurve $(T=280 \mathrm{~K})$ liegt in einem Temperaturbereich, in dem $U_{\omega}$ photoinduziert kleiner wird, $U_{3 \omega}$ dagegen größer. In Abb. 7.3 (c) und (d) ist die Temperaturabhängigkeit von $U_{\omega}$ und $U_{3 \omega}$ zusammenfassend aufgetragen. $U_{\omega, 3 \omega}$ ist für den belichteten (bei $t=100 \mathrm{~s}$ ) bzw. unbelichteten Fall (bei $t=10 \mathrm{~s}$ ) bei der jeweiligen Temperatur dargestellt.

In Abb. 7.4 dagegen wird der Diodenlaser zur Anregung verwendet. Bei diesen Messungen sind die Absolutwerte von $U_{\omega, 3 \omega}$ im Vergleich zu Abb. 7.3 unterschiedlich. Die Ursache hierfür liegt darin, dass zwischen diesen Messserien die Bonds auf der Struktur erneuert werden mussten. Dadurch ergeben sich die unterschiedlichen Spannungen bzw. Widerstände $\left(\Delta R_{\omega} \approx 30 \mathrm{k} \Omega\right)$. Ansonsten zeigt sich qualitativ die gleiche Abhängigkeit bei der Verwendung von dem Dioden- und dem Femtosekundenlaser. 


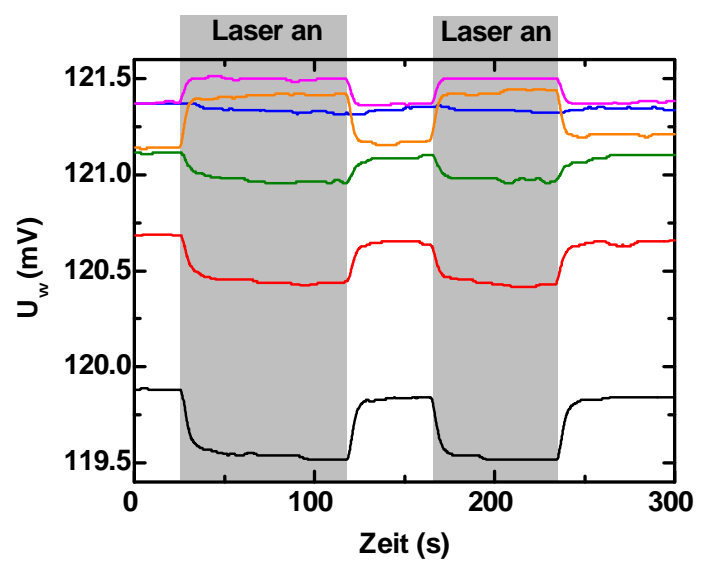

(a) $U_{\omega}(t)$

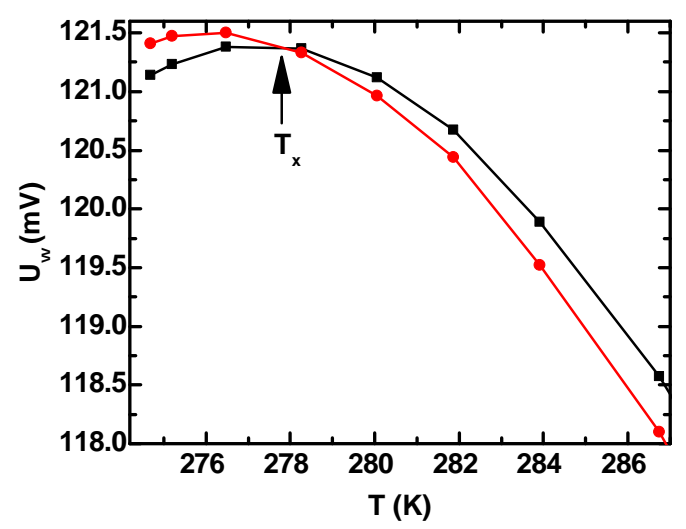

(c) $U_{\omega}(T)$

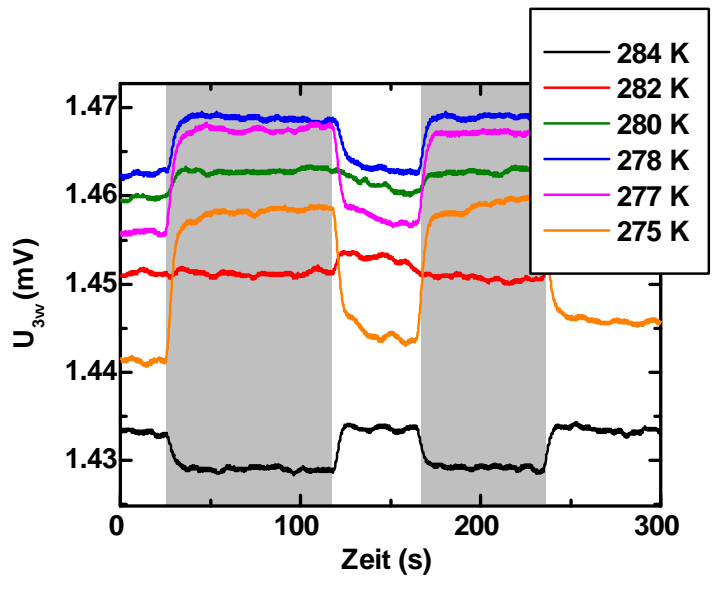

(b) $U_{3 \omega}(t)$

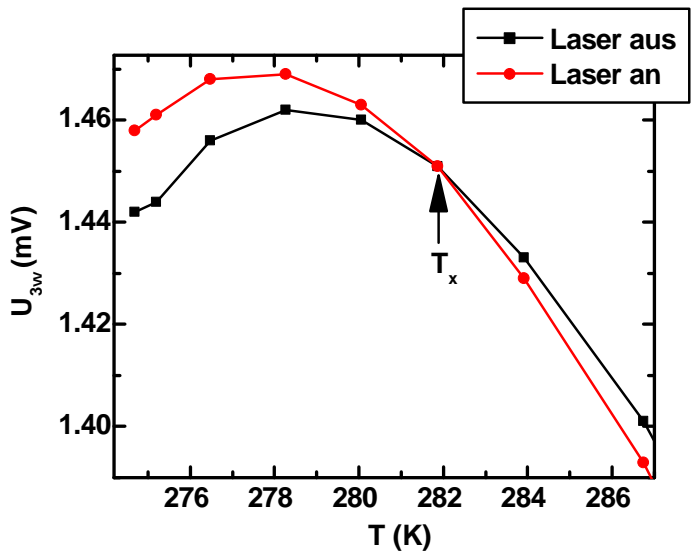

(d) $U_{3 \omega}(T)$

Abb. 7.3: Photoinduzierte Messungen von $U_{\omega, 3 \omega}$ : Anregung mit Femtolaser $(P=9,4 \mathrm{~mW})$. In der oberen Zeile sind $U_{\omega}$ (a) und $U_{3 \omega}$ (b) als Funktion der Zeit bei verschiedenen Temperaturen abgebildet. Die grau hinterlegten Bereiche markieren die Zeiten, in denen der Laser auf die Probe scheint. In der unteren Zeile sind $U_{\omega}$ (c) und $U_{3 \omega}(\mathrm{d})$ als Funkion der Temperatur gezeigt. Diese Kurven werden aus den Messungen in (a) und (b) ermittelt, indem $U_{\omega}$ und $U_{3 \omega}$ bei $t=10 \mathrm{~s}$ („Laser

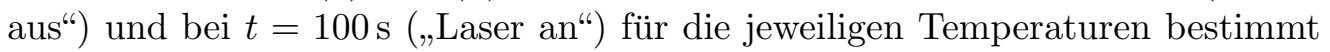
werden.

Aus den $U_{\omega, 3 \omega}(T)$-Kurven kann $T_{\mathrm{MI}}$ für den belichteten und unbelichteten Fall bestimmt werden. Diese Werte sind sowohl für den Diodenlaser als auch den Femtosekundenlaser in Tabelle 7.5 zusammengefasst. Als Mittelwert der Metall-Isolator-Übergangstemperatur ergibt sich aus allen Messungen $T_{\mathrm{MI}}=278 \mathrm{~K}$. Dieser Wert weicht erheblich von der mit dem PPMS bestimmten Metall-Isolator-Übergangstemperatur $\left(T_{\mathrm{MI}, \omega}=292 \pm 2 \mathrm{~K}\right.$ und $\left.T_{\mathrm{MI}, 3 \omega}=286 \pm 2 \mathrm{~K}\right)$ ab. Die Ursache hierfür liegt in der schlechten und auch unterschiedlichen thermischen Ankopplung des Pt1000 und der Probe an das Peltierelement I. Die Schicht ist lediglich durch das isolierende $\mathrm{SrTiO}_{3}$-Substrat im thermischen Kontakt mit dem Peltierelement. Der Unterschied von $6 \mathrm{~K}$ zwischen $T_{\mathrm{MI}, 3 \omega}$ und $T_{\mathrm{MI}, \omega}$ aus den PPMSMessungen wird in den Lock-In-Messungen nicht beobachtet. 


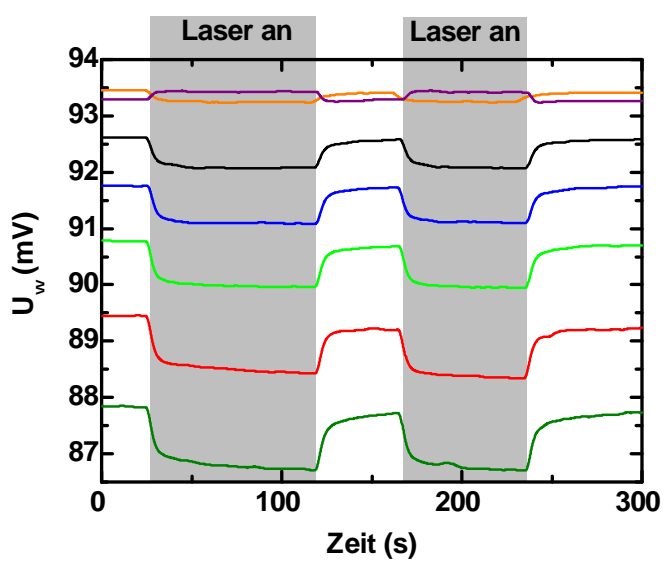

(a) $U_{\omega}(t)$

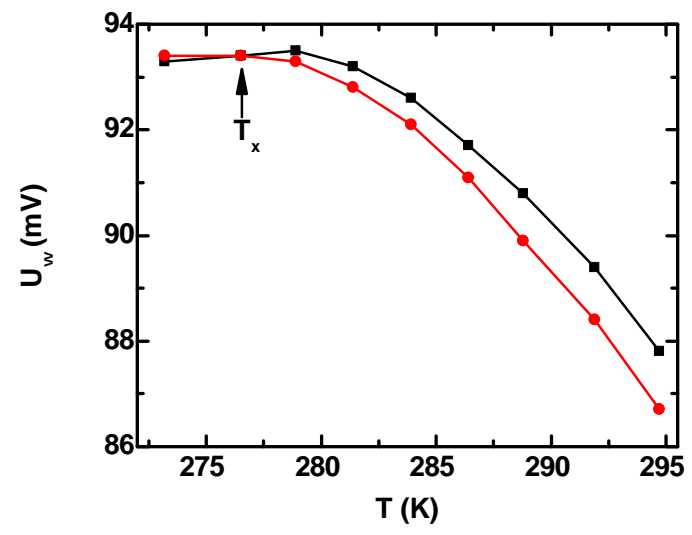

(c) $U_{\omega}(T)$

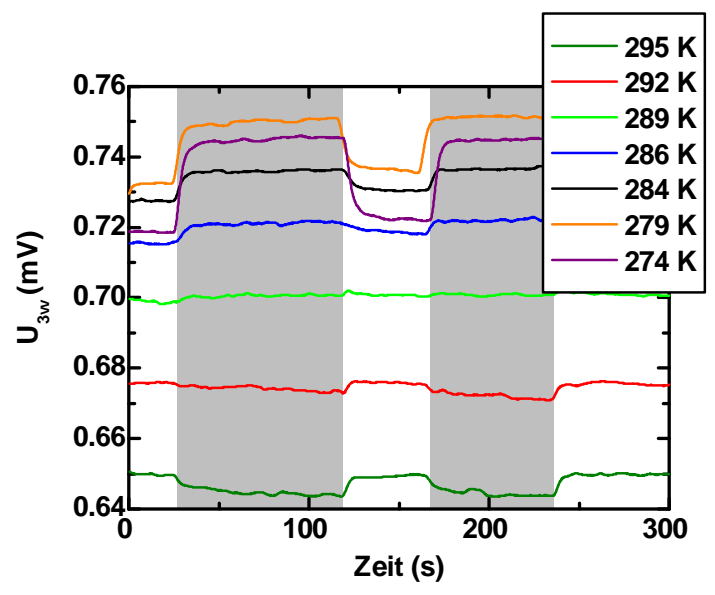

(b) $U_{3 \omega}(t)$

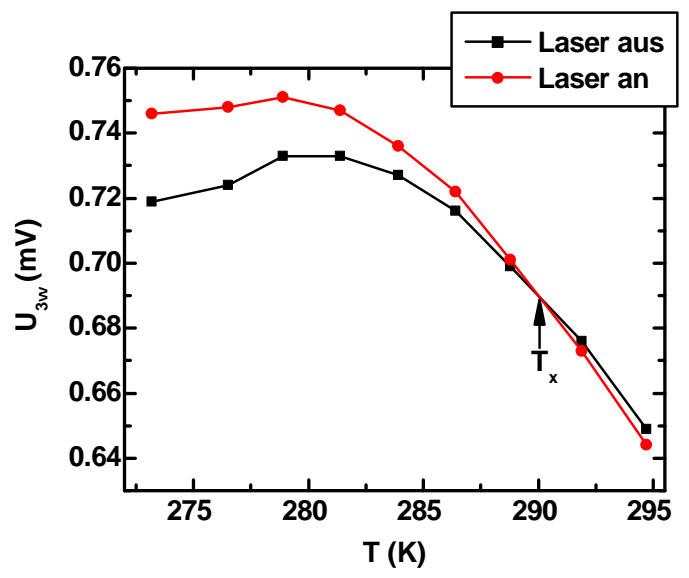

(d) $U_{3 \omega}(T)$

Abb. 7.4: Photoinduzierte Messungen von $U_{\omega, 3 \omega}$ : Anregung mit Diodenlaser $(P=$ $15,9 \mathrm{~mW})$.

In Tabelle 7.5 wird eine Temperatur $T_{x}$ angegeben. $T_{x}$ entspricht der Temperatur, bei der $\Delta U=U_{\text {Laser an }}-U_{\text {Laser aus }}$ einen Vorzeichenwechsel hat und ist in Abb. 7.3 und 7.4 (c) und (d) mit eingetragen. $T_{x}$ liegt für $\omega$ und $3 \omega$ bei unterschiedlichen Temperaturen. Im Temperaturbereich $T_{x, \omega}<T<T_{x, 3 \omega}$ wird $U_{\omega}$ durch Belichtung kleiner, während $U_{3 \omega}$ größer wird. Dieses ist für die Diodenlaser- genauso wie für die Femtosekundenlasermessungen der Fall.

Wie in Tabelle 7.5 ersichtlich, ist sowohl für die Femtosekundenlaser- als auch die Diodenlaseranregung $T_{x, 3 \omega}>T_{\mathrm{MI}, 3 \omega}$. Allerdings ist für den Diodenlaser $T_{x, 3 \omega}=290 \mathrm{~K}$, während für den Femtosekundenlaser $T_{x, 3 \omega}=282 \mathrm{~K}$. Unterschiede in den Messungen mit dem Dioden- und dem Femtosekundenlaser sind durch eine unterschiedliche mittlere Laserleistung $\left(P_{\text {Diodenlaser }}=15,9 \mathrm{~mW}, P_{\text {Femtolaser }}=9,4 \mathrm{~mW}\right)$ und verschiedene Wellenlängen $\left(\lambda_{\text {Diodenlaser }}=638 \mathrm{~nm}, \lambda_{\text {Femtolaser }}=795 \mathrm{~nm}\right)$ gegeben. Zusätzlich besteht der Unterschied zwischen einer CW- und einer Femtosekundenanregung. Auch der Durchmesser (beamwaist) des Laserstrahls auf der Struktur war unterschiedlich: Beim Femtosekundenlaser war der Strahldurchmesser $\tau=10 \ldots 15 \mu \mathrm{m}$, bei dem Diodenlaser dagegen $\tau=20 \ldots 30 \mu \mathrm{m}$. 


\begin{tabular}{lccc}
\hline & $\begin{array}{c}T_{\mathrm{MI}} \\
\text { Laser aus }\end{array}$ & $\begin{array}{c}T_{\mathrm{MI}} \\
\text { Laser an }\end{array}$ & $T_{x}$ \\
\hline \hline Femtolaser: & & & \\
$\omega$ & $277 \mathrm{~K}$ & $276 \mathrm{~K}$ & $278 \mathrm{~K}$ \\
$3 \omega$ & $278 \mathrm{~K}$ & $278 \mathrm{~K}$ & $282 \mathrm{~K}$ \\
\hline Diodenlaser: & & & \\
$\omega$ & $279 \mathrm{~K}$ & - & $277 \mathrm{~K}$ \\
$3 \omega$ & $280 \mathrm{~K}$ & $279 \mathrm{~K}$ & $290 \mathrm{~K}$ \\
\hline
\end{tabular}

Tabelle 7.5: $T_{\mathrm{MI}}$ und $T_{x}$ bestimmt aus $U_{\omega, 3 \omega}(T)$ der Abb. 7.3 und 7.4. Die Unsicherheit der Daten wird mit $\pm 2 \mathrm{~K}$ angenommen (resultierend aus der geringen Dichte der Messpunkte). Der absolute Fehler bei der Bestimmung der Schichttemperatur

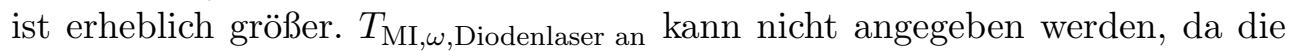
Temperatur nicht weit genug reduziert werden konnte.

\subsubsection{Zeitlicher Verlauf}

Die Messungen von $U_{\omega, 3 \omega}(t)$ zeigen insbesondere für das $U_{3 \omega}$-Signal ein sehr kleines Signal/Rausch-Verhältnis. Um das Signal/Rausch-Verhältnis zu verbessern, wurden in den bisher gezeigten Messungen sehr große Zeitkonstanten für die Lock-In-Verstärker gewählt $\left(\tau_{\mathrm{LI}}=1 \mathrm{~s}\right)$. Ein großer Anteil des Rauschens ist auf die Kontaktierung der Mikrostruktur mittels Bonddrähten zurückzuführen. Um die Bonddrähte auf dem Probenträger zu positionieren, werden sie mit Leitsilber auf den Kontakten des Probenträgers festgeklebt. Im Laufe der Messungen mussten die Bonddrähte immer wieder erneuert werden, da sie sich durch den Stickstoffstrom abgelöst hatten. Dieses hat sicherlich einen großen Anteil an dem schlechten Signal/Rausch-Verhältnis.

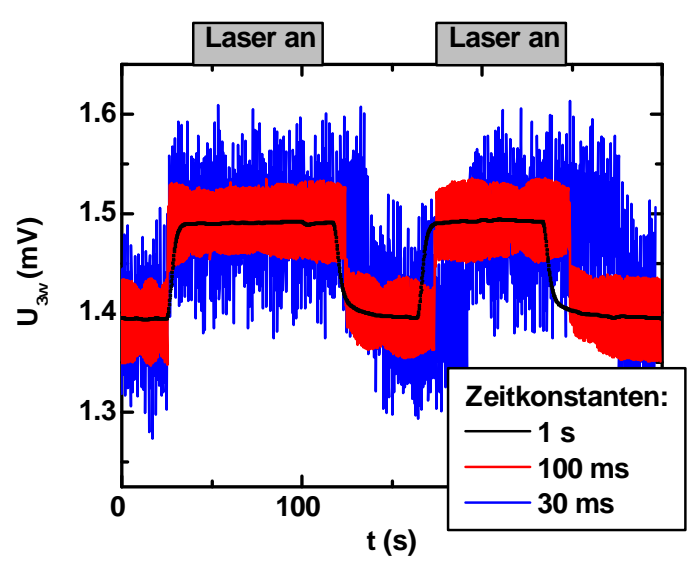

(a) $T=276 \mathrm{~K}$ (Diodenlaser $P=70 \mathrm{~mW})$

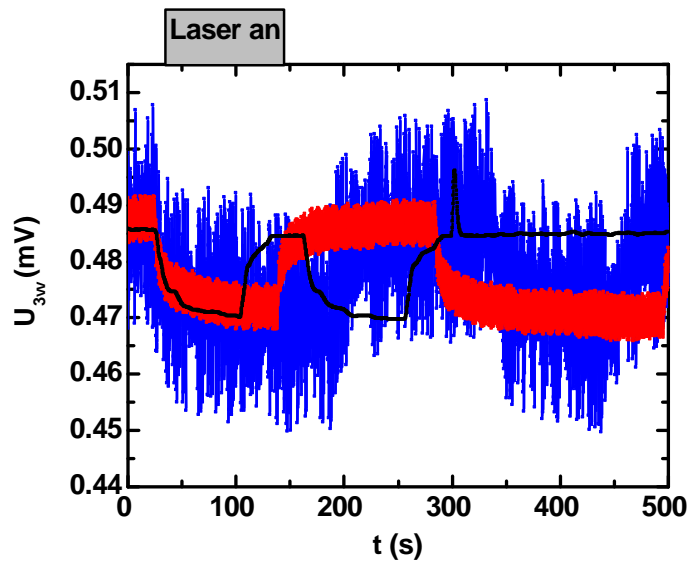

(b) $T=\mathrm{RT}($ Diodenlaser $P=15,9 \mathrm{~mW})$

Abb. 7.6: $U_{3 \omega}(t)$ für verschiedene Zeitkonstanten $\tau_{\mathrm{LI}}$. Die Zeitabhängigkeit des $U_{3 \omega}$-Signals kann nicht bestimmt werden, da die Messdaten bei kleinen Zeitkonstanten zu verrauscht sind. 

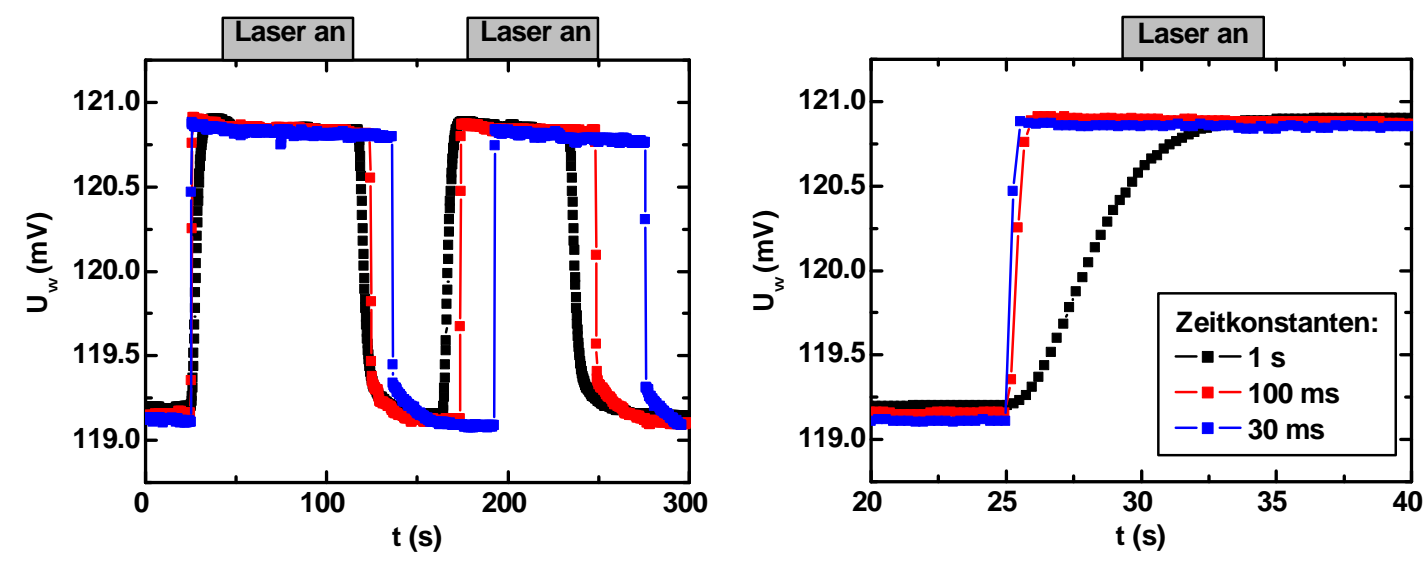

(a) $U_{\omega}(t)$ bei $T=276 \mathrm{~K}$; rechts: vergrößerter Bildausschnitt (Diodenlaser $P=70 \mathrm{~mW}$ )
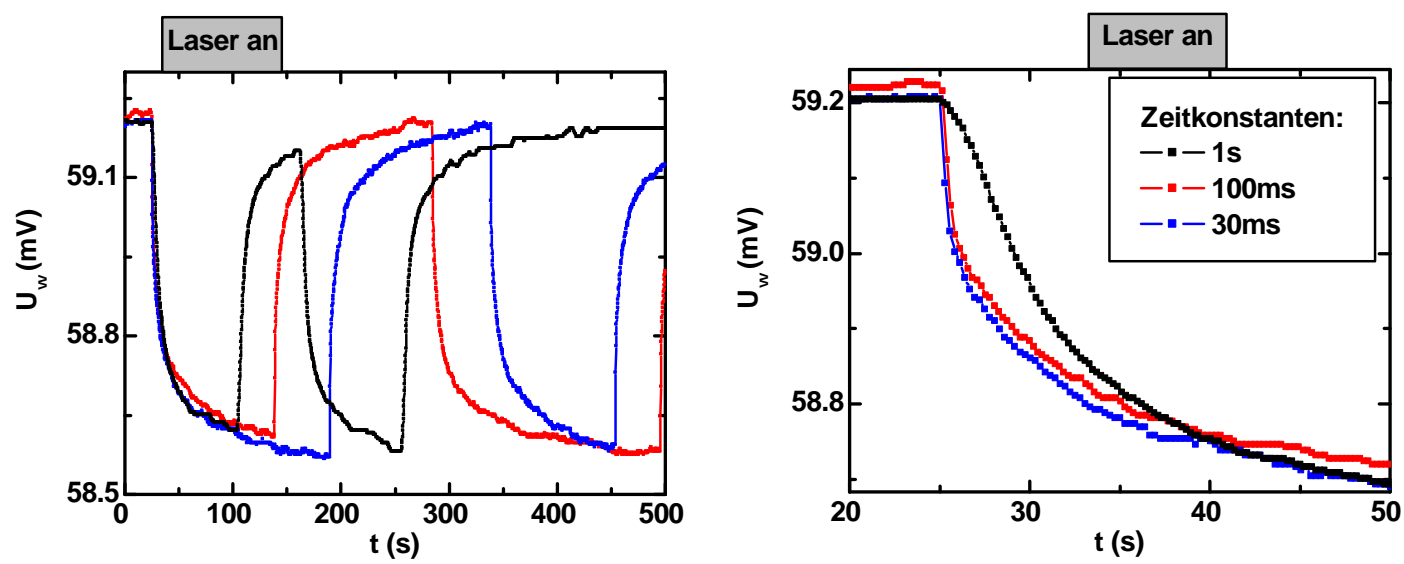

(b) $U_{\omega}(t)$ bei $T=\mathrm{RT}$; rechts: vergrößerter Bildausschnitt (Diodenlaser $P=15,9 \mathrm{~mW}$ )

Abb. 7.7: $U_{\omega}(t)$ für verschiedene Zeitkonstanten $\tau_{\mathrm{LI}}$ der Lock-In-Verstärker bei $T=$ $276 \mathrm{~K}$ (a) und $T=$ RT (b) (Messung ohne Kühlaufbau, daher keine Temperaturmessung).

Durch die Verwendung von relativ großen Zeitkonstanten $\left(\tau_{\mathrm{LI}}=1 \mathrm{~s}\right)$ wird die zeitliche Auflösung reduziert. Die Messgeschwindigkeit wird allerdings nicht ausschließlich durch die Zeitkonstanten $\tau_{\text {LI }}$ limitiert, auch die Auslesegeschwindigkeit des Computers mit dem verwendeten Messprogramm ist ein limitierender Faktor. Es wird nur etwa alle 0,3 s ein Punkt ausgelesen. Eine Variation der Zeitkonstanten ist in Abb. 7.6 und 7.7 zu sehen. Sowohl oberhalb als auch unterhalb von $T_{\mathrm{MI}}$ kann die Geschwindigkeit des photoinduzierten Prozesses lediglich für $U_{\omega}$ bestimmt werden. Eine Auswertung für $U_{3 \omega}$ ist nicht möglich, da aufgrund des geringeren Messsignals die Daten bei kleineren Zeitkonstanten zu verrauscht sind, wie in Abb. $7.6 \mathrm{zu}$ sehen ist. Des Weiteren ist zu beachten, dass nur $U_{\omega}(t)$-Messungen mit Diodenlaseranregungen vorgestellt werden.

Die zeitabhängige Messung von $U_{\omega}$ ist für $T=276 \mathrm{~K}$ in Abb. 7.7(a) gezeigt. Der zeitliche Verlauf des Signals ist für den Fall „Laser an“ und „Laser aus“ deutlich unterschiedlich. Im Fall „Laser an" ist der Anstieg des $U_{\omega}$-Signals schneller als die maximale Zeitaufösung von 0,3 s. Im Gegensatz dazu dauert es nach dem Blocken des Laserstrahls wesentlich länger, bis die Probe wieder im Gleichgewicht bzw. Ausgangszustand ist. Die gezeigten Daten können 


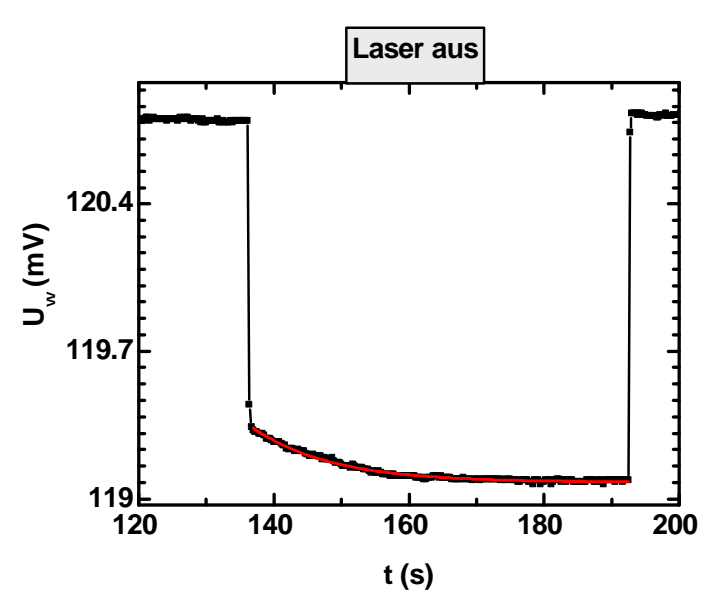

(a) $U_{\omega}(t)$ bei $T=276 \mathrm{~K}(P=70 \mathrm{~mW})$

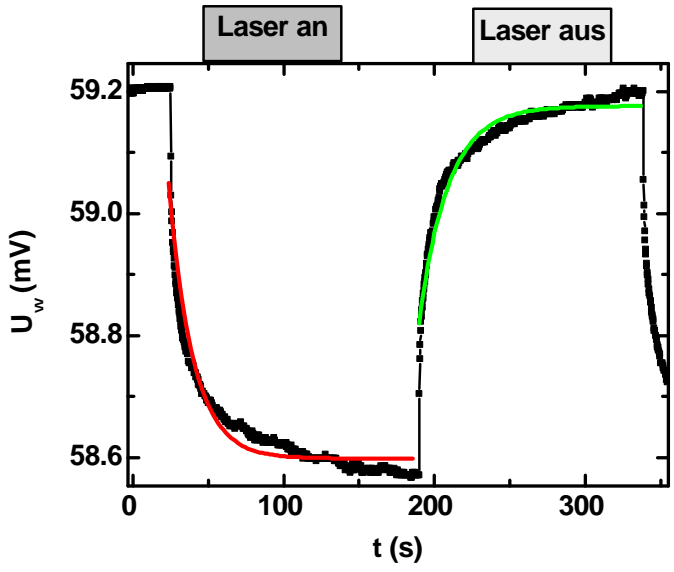

(b) $U_{\omega}(t)$ bei $T=\mathrm{RT}(P=15,9 \mathrm{~mW})$

Abb. 7.8: $U_{\omega}(t)$ mit exponentiellen Fits der Form $U_{\omega}(t)=A_{1} \cdot \exp \left(-x / t_{1}\right)+y_{0}$. Die Zeitkonstante der Lock-In-Verstärker beträgt $\tau_{\mathrm{LI}}=30 \mathrm{~ms}$.

\begin{tabular}{lccc}
\hline & $\mathrm{A}_{1}(\mathrm{mV})$ & $\mathrm{y}_{0}(\mathrm{mV})$ & $\mathrm{t}_{1}(\mathrm{~s})$ \\
\hline \hline$T=276 \mathrm{~K}$ & & & \\
Laser aus & $(25,6 \pm 4,7) \cdot 10^{3}$ & $119,1 \pm\left(9,5 \cdot 10^{-4}\right)$ & $11,96 \pm 0,2$ \\
\hline$T=\mathrm{RT}$ & & & \\
Laser an & $2,03 \pm 0,1$ & $58,6 \pm\left(1,4 \cdot 10^{-3}\right)$ & $15,9 \pm 0,4$ \\
Laser aus & $-6211 \pm 1431$ & $59,176 \pm\left(1,2 \cdot 10^{-3}\right)$ & $19,4 \pm 0,5$ \\
\hline
\end{tabular}

Tabelle 7.9: Fitparameter für die in Abb. 7.8 gezeigte Anpassung der Daten mit der Funktion $U_{\omega}(t)=A_{1} \cdot \exp \left(-x / t_{1}\right)+y_{0}$, die angegebenen Fehler beziehen sich auf die Güte des Fits.

durch eine Exponentialfunktion der Form $U_{\omega}(t)=A_{1} \cdot \exp \left(-x / t_{1}\right)+y_{0}$ angenähert werden (Abb. 7.8). Eine Übersicht über die Fitparameter ist in Tabelle 7.9 gegeben. Hierbei werden im Fall „Laser aus“ ( $T=276 \mathrm{~K})$ nur die Daten nach dem schnellen, nicht aufzulösenden Abfall berücksichtigt. In Abb. 7.7(b) sind Messungen bei $T=\mathrm{RT}\left(T>T_{\mathrm{MI}}\right)$ dargestellt. Es ist zu beachten, dass die Laserleistung des Diodenlasers nun bei $15,9 \mathrm{~mW}$ liegt (bei $T=276 \mathrm{~K}$ ist $P=70 \mathrm{~mW}$ ). Sowohl für den Fall „Laser an" als auch „Laser aus“ wird der sprunghafte Anstieg bzw. Abfall zu Beginn nicht beobachtet.

In Tabelle 7.9 sind die Ergebnisse der exponentiellen Fits zusammengefasst. Hierbei ergeben sich Zeitkonstanten in der Größenordnung von $10 \ldots 20 \mathrm{~s}$, wobei für $T=276 \mathrm{~K}$ bei den Fits der erste schnelle Prozess nicht mit berücksichtigt wird.

\subsection{Diskussion}

Der nachfolgende Abschnitt widmet sich der Diskussion der beschriebenen Messergebnisse. Der Gliederung der Messergebnisse folgend werden zunächst die temperaturabhängigen PPMS-Messungen (ohne Licht) analysiert, um im Anschluss die photoinduzierten Messungen der ersten und dritten harmonischen Spannunng zu diskutieren. 


\section{PPMS-Messungen}

In den PPMS-Messungen wird die Temperaturabhängigkeit der ersten und dritten harmonischen Spannung - ohne den Einfluss von Laserstrahlung - in der LBMO-Schicht untersucht. Ein Ergebnis dieser Messungen ist, dass $K\left(T_{\mathrm{MI}}\right)=-34 \mathrm{~dB}$ für die Mikrostruktur und $K\left(T_{\mathrm{MI}}\right)=-67 \mathrm{~dB}$ für die unstrukturierte LBMO-Schicht ist.

Die unterschiedlichen Koeffizienten resultieren (zu einem Teil) aus den verschiedenen Stromdichten $j$ der beiden Messungen, für die $j^{2} \propto U_{3 \omega} / U_{\omega} \equiv K^{\prime}$ gilt. [136] Das quadrierte Verhältnis der Stromdichten zwischen der strukturierten und unstrukturierten Schicht ist gegeben durch

$$
\left(\frac{j_{\text {unstrukturiert }}}{j_{\text {Mikrostruktur }}}\right)^{2}=\left(\frac{100 \mu \mathrm{A} /(1 \mathrm{~mm} \cdot 30 \mathrm{~nm})}{20 \mu \mathrm{A} /(30 \mu \mathrm{m} \cdot 30 \mathrm{~nm})}\right)^{2}=0,0225 .
$$

Im Vergleich dazu ist

$$
\frac{K_{\text {unstrukturiert }}^{\prime}}{K_{\text {Mikrostruktur }}^{\prime}}=\frac{10^{K_{\text {unstrukturiert }}}}{10^{K_{\text {Mikrostruktur }}}}=\frac{10^{-6,7}}{10^{-3,4}}=5 \cdot 10^{-4} .
$$

Die unterschiedlichen Stromdichten erklären demnach nur einen geringeren Unterschied der harmonischen Koeffizienten. Möglicherweise kann die zusätzliche Erhöhung des dritten harmonischen Koeffizienten in der Mikrostruktur durch lokale Unterschiede innerhalb der Probe erklärt werden. Zusätzlich ist es möglich, dass durch die Strukturierung die Ränder der Struktur beschädigt sind und daher die tatsächliche Stromdichte höher ist.

In der Veröffentlichung von Moshnyaga et al. [22] wurde $K(T)$ mittels Vierpunktmessungen an unstrukturierten Proben gemessen. Hierbei wird für eine $\mathrm{La}_{0,75} \mathrm{Ca}_{0,25} \mathrm{MnO}_{3} / \mathrm{MgO}-$ Probe (LCMO) ein Koeffizient von $K\left(T_{\mathrm{MI}}\right)=-60 \mathrm{~dB}$ gefunden $(I=100 \mu \mathrm{A} f=17 \mathrm{~Hz})$. Dieser ist vergleichbar mit der hier untersuchten (unstrukturierten) LBMO-Schicht.

In Abb. 7.2 wird $R_{3 \omega} / \mathrm{R}_{\omega}(T)$ für die unstrukturierte Schicht gezeigt. Nach Moshnyaga et al. kann hieraus der Anteil der korrelierten Polaronen bestimmt werden. [22] Dazu wird $R_{3 \omega} / R_{\omega}(T)$ mit der asymmetrischen doppelten Sigmoidalfunktion gefittet. Diese Funktion ist gegeben durch

$$
f(T)=y_{0}+A \cdot\left\{\frac{1}{1+e^{-\frac{T-T_{\mathrm{MI}}+w_{1} / 2}{w_{2}}}}\right\} \cdot\left\{1-\frac{1}{1+e^{-\frac{T-T_{\mathrm{MI}}-w_{1} / 2}{w_{3}}}}\right\} .
$$

Dabei geben die Parameter $w_{2} \neq w_{3}$ die Breite der beiden Übergangsregime links und rechts des Metall-Isolator-Übergangs an. Der Anteil der korrelierten Polaronen $N_{\mathrm{CP}}$ an der Gesamtzahl der Ladungsträger $N$ wird durch den Fitparameter $A=N_{\mathrm{CP}} / N\left(T_{\mathrm{MI}}\right)$ bestimmt.

Der Fit an die Messdaten der LBMO-Schicht ist in Abb. 7.2 eingezeichnet. Für den Fitparameter $A$ ergibt sich $A=N_{\mathrm{CP}} / N\left(T_{\mathrm{MI}}\right)=3,2 \cdot 10^{-7}\left( \pm 2 \cdot 10^{-8}\right) .{ }^{1}$ Im Vergleich dazu wurde für die LCMO-Probe $N_{\mathrm{CP}} / N\left(T_{\mathrm{MI}}\right)=(1 \ldots 2) \cdot 10^{-6}$ ermittelt. [22] Damit ist die Anzahl der korrelierten Polaronen in der LBMO-Schicht fast um einen Faktor zehn kleiner als in der LCMO-Schicht. Ein Unterschied zwischen LCMO und LBMO ist eine modifizierte Perowskit-Kristallstruktur. In der Literatur [137] wird für $\mathrm{La}_{2 / 3} \mathrm{Ba}_{1 / 3} \mathrm{MnO}_{3}$ in der Nähe

\footnotetext{
${ }^{1}$ Die übrigen Fitparameter sind $y_{0}=1,9 \cdot 10^{-23} \pm 4 \cdot 10^{-10} ; T_{\mathrm{MI}}=285,5 \pm 1,2 \mathrm{~K} ; w_{1}=21 \pm 3 \mathrm{~K}$;
} $w_{2}=5,2 \pm 0,2 \mathrm{~K} ; w_{3}=19,3 \pm 0,7 \mathrm{~K}$. 
der Curie-Temperatur hauptsächlich die $\mathrm{R} \overline{3} \mathrm{c}$-Struktur gefunden, ${ }^{2}$ diese ist ungünstiger für Jahn-Teller-Polaronen als die Pnma-Kristallstruktur der LCMO-Schicht. Daher entspricht die reduzierte korrelierte Polaronen-Konzentration qualitativ den Erwartungen. Allerdings ist $\mathrm{zu}$ beachten, dass das $\mathrm{La}_{1-x} \mathrm{Ba}_{x} \mathrm{MnO}_{3}$-System sehr empfindlich auf die Ordnung der Kationen reagiert (die La- und Ba-Ionen haben relativ unterschiedliche Ionenradien). Daher können sehr unterschiedliche Eigenschaften auch bei nominell gleich dotierten Proben auftreten.

\section{Photoinduzierte Änderungen - Temperaturabhängigkeit}

Im Folgenden werden die photoinduzierten Messungen diskutiert, welche im Abschnitt 7.2.1 beschrieben werden. Hierbei handelt es sich um die temperaturabhängigen Messungen von $U_{3 \omega}$ und $U_{\omega}$ mit und ohne Laseranregung durch den Femtosekundenlaser (Abb. 7.3) und den Diodenlaser (Abb. 7.4).

Bei der Interpretation dieser Messdaten müssen insbesondere Heizeffekte berücksichtigt werden. Induziert der Laser in der Schicht eine Temperaturänderung von $\Delta T$, so verändert sich der Probenwiderstand von $R\left(T_{1}\right)$ zu $R\left(T_{2}=T_{1}+\Delta T\right)$. Unter der Annahme, dass die Temperaturänderung (bei konstanter Laserleistung) im hier untersuchten Temperaturbereich gleich ist, können die belichtete und unbelichtete $R(T)$-Kurve durch Verschiebung auf der Temperaturachse aufeinander geschoben werden. In Abb. 7.10 ist (am Beispiel der Diodenlasermessungen) diese auf der Temperaturachse verschobene Kurve $U_{\text {Laser an }}(T+\Delta T)$ (mit $\Delta T=2 \mathrm{~K}$ ) eingezeichnet. Die Temperaturverschiebung $\Delta T$ wird so gewählt, dass die Übereinstimmung zwischen belichteter und unbelichteter Kurve möglichst groß ist. Es zeigt sich, dass dieses für $U_{\omega}(T)$ mit $\Delta T=2 \mathrm{~K}$ relativ gut gelingt; für $U_{3 \omega}(T)$ dagegen nicht. Bei der Messreihe mit dem Femtosekundenlaser (Laserleistung $P=9,4 \mathrm{~mW}$ ) reicht eine Temperaturverschiebung von $\Delta T=1 \mathrm{~K}$ aus, um die $U_{\omega}(T)$-Kurven des belichteten und unbelichteten Falls aufeinander zu legen. Dieses ist wiederum für die $U_{3 \omega}(T)$-Kurven nicht möglich.

Woraus resultiert die erhöhte, dritte harmonische Spannung bei Anregungen mit den Lasern? Moshnyaga et al. 22] bringen die im Bereich des Metall-Isolator-Übergangs erhöhte, dritte harmonische Spannung mit der Konzentration von korrelierten Polaronen in Verbindung. Es stellt sich die Frage, ob korrelierte Polaronen durch Anregungen mit den verwendeten Lasern entstehen können. Die Wellenlängen der Laser liegen bei 1,96 und 1,56 eV (Dioden- und Femtosekundenlaser). Welche optisch induzierten Übergänge gibt es in diesem Energiebereich?

Eine Arbeit an $\mathrm{La}_{0,7} \mathrm{Sr}_{0,3} \mathrm{MnO}_{3^{-}}, \mathrm{La}_{0,7} \mathrm{Ca}_{0,3} \mathrm{MnO}_{3^{-}}$und $\mathrm{Nd}_{0,7} \mathrm{Sr}_{0,3} \mathrm{MnO}_{3}$-Schichten findet drei Maxima in der optischen Leitfähigkeit, bei $1 \mathrm{eV}, 3 \mathrm{eV}$ und $4 \mathrm{eV}$. [138] Bei $1 \mathrm{eV}$ finden Quijada et al. ein breites Maximum für alle drei Filme in der Nähe und oberhalb der Curie-Temperatur. [138] Dieses Maximum wird durch von Photonen induziertes Hüpfen von kleinen Jahn-Teller-Polaronen erklärt. Dieser Übergang scheint energetisch möglich; allerdings ist unklar, welchen Einfluss eine veränderte Beweglichkeit der Polaronen auf die dritte harmonische Spannung haben könnte. Das Maximum bei $3 \mathrm{eV}$ wird mit $\mathrm{e}_{g^{-}} \mathrm{e}_{g^{-}}$ Übergängen assoziiert, wobei der Endzustand eine antiparallele Stellung der $\mathrm{e}_{g}$-Elektronen und der $\mathrm{t}_{2 g}$-Rumpfspins hat. Der Übergang bei $4 \mathrm{eV}$ entspricht einem LadungstransferÜbergang (charge transfer transition) zwischen $\mathrm{O}_{2 p}$ und $\mathrm{Mn}_{e_{g}}$.

\footnotetext{
${ }^{2}$ Beznosov et al. finden in ihren $\mathrm{La}_{2 / 3} \mathrm{Ba}_{1 / 3} \mathrm{MnO}_{3}$-Proben unterhalb der Curie-Temperatur $\left(\mathrm{T}_{\mathrm{C}}=314 \mathrm{~K}\right)$ hauptsächlich die R $\overline{3}$ c-Struktur, allerdings auch einen Anteil an orthorhombischer Imma-Struktur. Bei einer Temperatur von $T_{e}=200 \mathrm{~K}$ kommt es zu einem (fast vollständigen) strukturellen Phasenübergang zu der Imma-Phase. [137]
} 


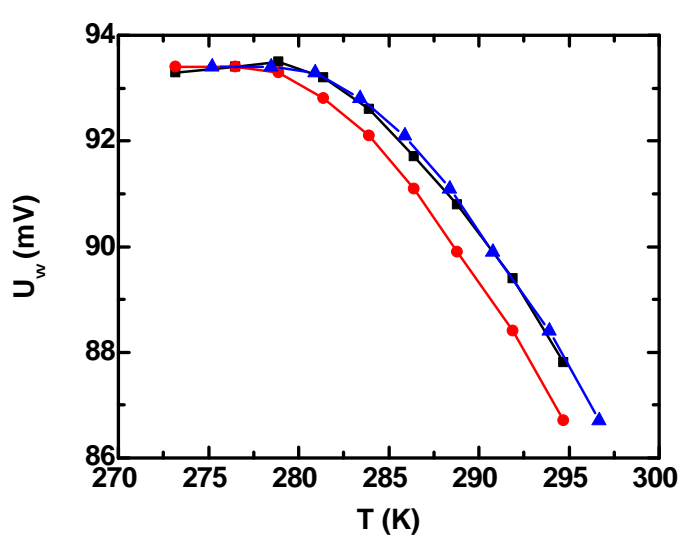

(a) Temperaturabhängigkeit von $U_{\omega}$

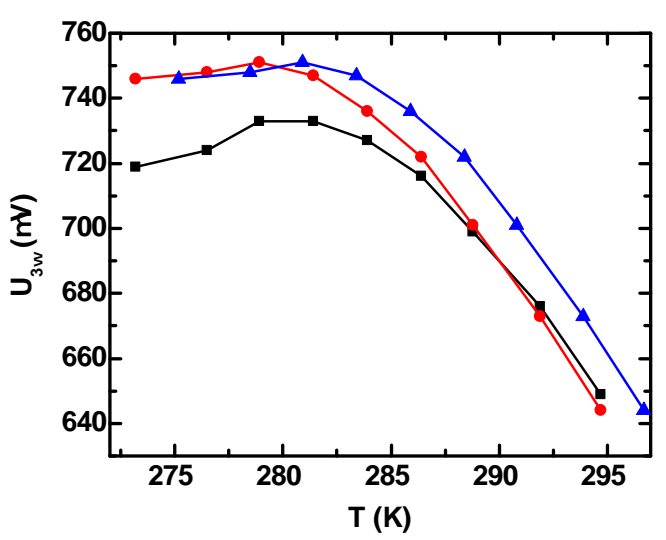

(b) Temperaturabhängigkeit von $U_{3 \omega}$

Abb. 7.10: $U_{\omega, 3 \omega}(T)$ mit und ohne Belichtung; zusätzlich ist die um $\Delta T=2 \mathrm{~K}$ verschobene, „Laser an"-Kurve eingezeichnet (Diodenlaser, $P=15,9 \mathrm{~mW}$ ).

Coey et al. finden den Ladungstransfer-Übergang bei $3,1 \mathrm{eV}$ und einen zweiten Übergang bei $1,7 \mathrm{eV}$. [52, 139] Hier wird der Übergang bei $1,7 \mathrm{eV}$ mit dem Übergang von einem $\mathrm{Mn}_{\mathrm{t}_{2 g}}$ in einen $\mathrm{Mn}_{\mathrm{e}_{g}}$-Zustand erklärt. Bei diesen Übergängen $\left(\mathrm{O}_{2 \mathrm{p}} \rightarrow \mathrm{Mn}_{\mathrm{e}_{g}}\right.$ und $\mathrm{Mn}_{\mathrm{t}_{2 g}} \rightarrow$ $\mathrm{Mn}_{\mathrm{e}_{g}}$ ) werden Elektronen in das $\mathrm{e}_{g}$-Band gehoben. Diese können sich möglicherweise mit anderen Jahn-Teller-Polaronen zu korrelierten Polaronen zusammenfinden (um die elastische Verzerrungsenergie zu minimieren) und damit zu einer im Metall-Isolator-Übergang erhöhten, dritten harmonischen Spannung beitragen.

An dieser Stelle sind weiterführende Experimente notwendig. So sollte in einem nächsten Schritt der Zusammenhang zwischen Wellenlänge und dritter harmonischer Spannung identifiziert werden. Des Weiteren kann mit dem vorhandenen Messaufbau lediglich in einem kleinen Temperaturbereich in der Nähe des Metall-Isolator-Übergangs gemessen werden. Daher bleibt der Tieftemperaturbereich bzw. das Verhalten der ferromagnetischen, metallischen Phase unbekannt. Wird in einem Aufbau (der tiefere Temperaturen erreicht) $U_{\omega, 3 \omega}(T)$ mit und ohne Laserlicht gemessen, so kann möglicherweise mit der von Moshnyaga et al. 22] angegebenen Fitfunktion die Anzahl der korrelierten Polaronen für den belichteten und unbelichteten Fall bestimmt werden.

\section{Photoinduzierte Änderungen - Zeitlicher Verlauf}

Der zeitliche Verlauf der photoinduzierten Änderungen der ersten harmonischen Spannung wird im Unterkapitel 7.2 .2 vorgestellt. Bei tiefen Temperaturen $(T=276 \mathrm{~K}, P=70 \mathrm{~mW})$ wird im Fall „Laser an" und „Laser aus" ein sprunghafter Anstieg bzw. Abfall des Signals beobachtet. Im Fall „Laser an“ ist die photoinduzierte Änderung schneller, als durch die begrenzte Zeitauflösung des Messaufbaus untersucht werden kann (es kann lediglich alle $0,3 \mathrm{~s}$ ein Messpunkt ausgelesen werden). Oberhalb der Metall-Isolator-Übergangstemperatur $(P=15,9 \mathrm{~mW})$ werden langsamere, photoinduzierte Änderungen beobachtet. Die $U_{\omega}(t)$-Kurven werden mittels einer Exponentialfunktion $\left(U_{\omega}(t)=A_{1} \cdot \exp \left(-x / t_{1}\right)+y_{0}\right)$ gefittet, die Ergebnisse der Fitparameter sind in Tabelle 7.9 zusammengefasst. Der Fitparameter $t$ liegt dabei in der Größenordnung von $t=10 \ldots 20 \mathrm{~s}$. Hierbei kann allerdings für $T=276 \mathrm{~K}$ nur der Fall „Laser aus“ und in diesem nur ein Teil der Daten berücksichtigt werden (siehe Abb. 7.8(a)) . 
Die Größenordnung der Zeitkonstanten aus diesen exponentiellen Fits stimmt mit den Ergebnissen von Smolyaninova et al. [140] und Moshnyaga et al. [141] überein. Smolyaninova et al. [140] untersuchten an $\mathrm{La}_{0,7} \mathrm{Ba}_{0,3} \mathrm{MnO}_{3}$-Schichten die photoinduzierte Leitfähigkeitsänderung in der ferromagnetischen Phase $\left(T<T_{\mathrm{MI}, \mathrm{C}}\right)$. Hierbei wurde ein Argonionenlaser (CW-Betrieb, $P=150 \mathrm{~mW}, E=2,5 \mathrm{eV}$ ) verwendet, der eine photoinduzierte Erhöhung des Widerstandes bewirkt. Die Relaxation - nach Ende der Laserbeleuchtung - wird bei unterschiedlichen Temperaturen gemessen. Bei höheren Temperaturen ist die Zeitkonstante kleiner. Die höchste Temperatur, die in der Veröffentlichung angegeben wird, ist $T=250 \mathrm{~K}$, hier ist die Zeitkonstante $\tau=20 \mathrm{~s}$ und damit in der gleichen Größenordnung wie in den hier gezeigten Messungen. Der Erklärungsansatz von Smolyaninova et al. ist eine photoinduzierte Zerstörung der ferromagnetischen Phase. Die entstehende Phase wird nicht weiter charakterisiert, sondern als magnetisch ungeordnete, photoinduzierte, resistive Phase bezeichnet.

Moshnyaga et al. [141] untersuchten den Einfluss von Femtosekundenlaser-Strahlung $(\lambda=$ $760 \mathrm{~nm}, \Delta t_{P u l s}=100 \mathrm{fs}$, durchschnittliche Leistung bis zu $1 \mathrm{~W}$ ) in $\mathrm{La}_{0,7} \mathrm{Ca}_{0,3} \mathrm{MnO}_{3}$-Schichten (LCMO). Für $T<T_{\mathrm{MI}}$ wird - wie in den hier beschriebenen Ergebnissen - photoinduziert der Widerstand der ferromagnetischen, metallischen Phase erhöht; oberhalb von $T_{\mathrm{MI}}$ verringert sich der Widerstand durch Bestrahlung. Als typische Relaxationszeit wird $\tau=1 \ldots 30$ s angegeben. Dieses liegt wiederum in der gleichen Größenordnung wie in den hier vorgestellten Messungen. Als Erklärung für $T<T_{\mathrm{MI}}$ geben Moshnyaga et al. [141] eine photoinduzierte kooperative Jahn-Teller-Transformation (in kleinen Clustern) an. Der Laser erhöht dabei die Größe bzw. Anzahl der ladungsgeordneten, antiferromagnetischen Cluster. Dieser Erklärungsansatz stimmt auch mit den hier gezeigten, temperaturabhängigen $U_{3 \omega}$-Messungen überein. Die erhöhte dritte harmonische Spannung im Bereich des Übergangs kann möglicherweise mit einer erhöhten Konzentration von korrelierten Polaronen erklärt werden.

Bei tiefen Temperaturen wird zusätzlich ein sprunghafter Anstieg bzw. Abfall in $U_{\omega}(t)$ beobachtet. Weiterhin zeigt sich ein Unterschied zwischen dem Fall "Laser aus" und „Laser an". Allerdings war die Laserleistung bei $T=276 \mathrm{~K}$ mit $P=70 \mathrm{~mW}$ deutlich größer als bei den Messungen $T>T_{\mathrm{MI}}(P=15,9 \mathrm{~mW})$. Daher kann an dieser Stelle nicht eindeutig nachgewiesen werden, ob dieser Effekt auf den Einfluss der Temperatur oder der Laserleistung zurückzuführen ist. Deswegen sind hier weitere Untersuchungen, die zum einen die Abhängigkeit von der Laserleistung untersuchen und zum anderen eine größere Zeitauflösung ermöglichen, notwendig und auch sehr vielversprechend.

\subsection{Zusammenfassung}

In diesem Kapitel wird der Einfluss von Laserlicht auf die erste und dritte harmonische Spannung in einer LBMO-Schicht untersucht. Temperaturabhängige Messungen zeigen, dass die $U_{3 \omega}(T)$-Kurven im Fall „Laser an“ und „Laser aus“ nicht durch Verschiebung auf der Temperaturachse übereinander gelegt werden können. Dieses legt die Überlegung nahe, dass die photoinduzierte Änderung des $U_{3 \omega}$-Signals nicht durch einen reinen Heizprozess erklärt werden kann. Möglicherweise regt das Laserlicht Elektronen in das $\mathrm{e}_{g}$-Band an, wodurch Polaronen oder korrelierte Polaronen entstehen können. Eine systematische Untersuchung, welchen Einfluss die Laserleistung, die Wellenlänge und der Unterschied zwischen CW-Betrieb und Femtosekundenpulsen auf die Temperatur $T_{x}$ haben, muss durchgeführt werden, um den zugrunde liegenden Mechanismus weiter zu beleuchten.

In einer weiteren Versuchreihe wird die Zeitabhängigkeit der photoinduzierten Änderung untersucht. Die größte Schwierigkeit hierbei ist das sehr schlechte Signal/Rausch-Verhält- 
nis, das eine Untersuchung der Zeitabhängigkeit von $U_{3 \omega}(t)$ nicht erlaubt. Für $U_{\omega}(t)$ zeigt sich für Temperaturen von $T=276 \mathrm{~K}$ im Fall „Diodenlaser an“ eine photoinduzierte Änderung, die schneller ist als die Zeitauflösung des Aufbaus $(0,3 \mathrm{~s})$. Für $T>T_{\mathrm{MI}}$ wird kein schneller Prozess beobachtet. Es bleibt die Frage offen, in wiefern hier die sehr unterschiedlichen Laserleistungen ( $P=15,9 \mathrm{~mW}$ im Vergleich zu $P=70 \mathrm{~mW})$ eine Rolle spielen. Wird der schnelle Anstieg zu Beginn in den Messungen bei tieferen Temperaturen vernachlässigt, ergeben sich Zeitkonstanten in der Größenordnung von $10 \ldots 20$ s. Diese liegen in der gleichen Größenordnung der Ergebnisse von Smolyaninova et al. [140] und Moshnyaga et al. [141].

Der Einfluss von Heizeffekten auf das $U_{\omega^{-}}$und $U_{3 \omega^{-}}$Signal muss weiter untersucht werden. Eine Messmöglichkeit hierfür besteht in der Variation der Laserleistung direkt unterhalb des Metall-Isolator-Übergangs. Bei einem reinen Heizprozess sollte es möglich sein, durch eine genügend große Laserleistung die Temperatur der Schicht über $T_{\mathrm{MI}}$ zu heizen. Dadurch müsste das $U_{\omega^{-}}$(und auch das $U_{3 \omega^{-}}$) Signal bei kleinen Leistungen erst ansteigen und bei größeren Laserleistungen wieder abfallen. Sind allerdings nicht nur Heizprozesse ursächlich, wird ein anderes Verhalten erwartet, etwa eine Sättigung des $U_{\omega, 3 \omega}$-Signals. Dieses Experiment war in dem vorhandenen Aufbau nicht möglich, da die Temperatur nicht genügend stabil gehalten werden konnte.

Insgesamt bleiben am Ende dieses Abschnittes etliche Fragen offen, insbesondere wenn bedacht wird, dass die gezeigten Messungen an lediglich einer Struktur auf einer Probe durchgeführt wurden. Allerdings scheinen die gezeigten Ergebnisse inkonsistent mit einem reinen Heizprozess zu sein. Es treten zusätzliche Nichtlinearitäten auf, die besonders empfindlich durch eine Messung der dritten harmonischen Spannung untersucht werden können. Die hier vorgestellten Experimente ebnen somit den Weg für zukünftige Messungen von photoinduzierten, höheren harmonischen Spannungen. Untersuchungen dieser Art können dazu beitragen, dass die zugrundeliegende Physik eines stark korreliertes Elektronensystems, wie es die Manganate sind, weiter beleuchtet und verstanden wird. 



\section{Zusammenfassung und Ausblick}

Die gemischtvalenten Manganoxide bieten ein faszinierendes Forschungsgebiet, deren Verständnis trotz zwanzig Jahren intensiver Forschung die moderne Festkörperphysik immer noch vor große Herausforderungen stellt. Aufgrund der starken Elektron-PhononWechselwirkung und der starken Korrelation der Elektronen untereinander kann eine Vielzahl von elektronischen, magnetischen und strukturellen Phasen und Phasenübergänge als Funktion von unterschiedlichen Kontrollparametern beobachtet werden. Neben der Dotierung können auch Temperatur-, Druck-, Magnetfeld-, elektrisches Feld- und photoinduzierte Phasentransformationen beobachtet werden.

In dieser Arbeit werden als externe Stimuli der Effekt eines elektrischen Feldes und der Einfluss von Licht auf die elektrischen Transporteigenschaften analysiert. Ein elektrisches Feld bewirkt beim bipolaren Widerstandsschalten eine remanente Widerstandsänderung. Diese wird im Rahmen eines lokalen strukturellen Phasenübergangs diskutiert. Des Weiteren wird der Einfluss einer Laseranregung erforscht. Hierbei werden durch Messungen von ersten und dritten harmonischen Spannungen Rückschlüsse auf die Konzentration von korrelierten Polaronen gezogen. In beiden Fällen wird der Einfluss der externen Anregung auf die elektronischen und damit verbundenen strukturellen Änderungen in den Manganatschichten durch elektrische Transportmessungen ergründet.

Im Kontext dieser Arbeit werden Manganatschichten mittels der Metallorganischen Aerosol Depositionstechnik präpariert. Es werden insbesondere $\mathrm{La}_{0,7} \mathrm{Sr}_{0,3} \mathrm{MnO}_{3}$-Schichten auf $\mathrm{Al}_{2} \mathrm{O}_{3}$-Substraten hergestellt, die ein polykristallines, nanokolumnares Wachstum zeigen. Eine Standardcharakterisierung bezüglich der elektronischen, magnetischen und strukturellen Eigenschaften dieser Schichten wird im Kap. 5 gezeigt. Hierbei wird der Einfluss der Defektstruktur der polykristallinen Schichten durch einen Vergleich mit epitaktischen Schichten $\left(\mathrm{La}_{0,7} \mathrm{Sr}_{0,3} \mathrm{MnO}_{3} / \mathrm{MgO}\right)$ deutlich. Insbesondere Untersuchungen an nanokolumnaren Proben mit Schichtdicken zwischen $10 \ldots 300 \mathrm{~nm}$ weisen auf das aus der Literatur bekannte core shell-Modell, in welchem der Kornhülle und dem Korninneren unterschiedliche physikalische Eigenschaften zugeordnet werden.

Eine Untersuchung der remanenten, bipolaren Schalteffekte in den polykristallinen Manganatschichten wird im Kap. 6 vorgestellt. Dem Widerstandsschalten wird besonders im Rahmen von nichtflüchtigen Speichermedien ein großes technisches Anwendungspotenzial zugesprochen. Je weiter die Miniaturisierung voranschreitet, desto wichtiger wird der Ein- 
fluss der lokalen Defektstruktur. Die in dieser Arbeit durchgeführten Schaltexperimente mittels C-AFM und an Mikrostrukturen weisen sowohl auf der lokalen als auch globalen Skala auf die herausragende Bedeutung der den nanokolumnaren Schichten zugrunde liegenden Defektstrukturen hin. Die C-AFM Messungen zeigen deutlich, dass der Schalteffekt insbesondere im Bereich der Korngrenzen begünstigt ist. Weiterhin demonstrieren sie den Unterschied zwischen einer $44 \mathrm{~nm}$ dünnen und $300 \mathrm{~nm}$ dicken LSMO-Schicht. Während die dünne Schicht erst durch ein elektrisches Feld in den LRS geschaltet werden kann, ist im Ausgangszustand der dicken Schicht schon ein signifikanter Anteil im tiefohmigen Zustand. In den Experimenten an den Mikrostrukturen tragen stets eine Vielzahl von Manganatsäulen zum elektrischen Transportverhalten bei. Hierbei wird der Einfluss der Temperatur, des Magnetfeldes und der maximalen Schaltspannung auf das Widerstandsschalten untersucht. Bemerkenswert in diesen Mikrostrukturmessungen sind die vielen remanenten Zwischenstufen zwischen dem HRS und LRS.

Die Ergebnisse der C-AFM und Mikrostrukturmessungen können gut im Rahmen einer verallgemeinerten Energielandschaft beschrieben werden, in der HRS und LRS in einem Doppelmuldenpotential dargestellt werden. Aus den Messergebnissen der vorliegenden Arbeit werden die Besonderheiten und Unterschiede zwischen der Schichtoberfläche und den Korngrenzen abgeleitet. Diese zeigen unterschiedlich hohe Energiebarrieren zwischen HRS und LRS. Für die nanokolumnaren Schichten ergibt sich zusätzlich noch eine weitere Unterteilung im Bereich der Korngrenzen in Abhängigkeit der Schichtdicke.

Als zugrunde liegender mikroskopischer Mechanismus des Schaltens wird ein lokaler struktureller Übergang im Bereich der Kornhüllen ( shell) diskutiert. Hierbei wird für den HRS eine niedersymmetrische, isolierende Phase in der Hülle angenommen. Diese wird unter dem Einfluss eines elektrischen Feldes beim Schalten in die höhersymmetrische, leitfähigere $\mathrm{R} \overline{3}$ c-Struktur des Korninneren transformiert. Für dieses Modell und gegen eine Sauerstoffleerstellen-Diffusion - wie sie eine große Rolle in vielen aktuellen Veröffentlichungen spielt - sprechen insbesondere die temperaturabhängigen Messungen und die lokale Geometrie beim Schalten.

Eine Beschreibung und Simulation der Mikrostrukturmessungen gelingt gut im Kontext eines nanokolumnaren Netzwerks aus widerstandsschaltenden Elementen. In den Mikrostrukturexperimenten werden viele remanente Widerstandszustände beobachtet: Diese können als nichtflüchtige Multilevel-Speicherzellen von technologischem Interesse sein. Ebenso von technischer Relevanz ist möglicherweise die vorgestellte Kombination von Magnetowiderstands- und Widerstandsschaltmessungen, die eine mögliche Anwendung als Sensor mit Speicherfunktion für magnetische und elektrische Felder bieten können. Allerdings muss hier angemerkt werden, dass die vorgestellten Messungen bei Temperaturen weit unterhalb von Raumtemperatur durchgeführt wurden.

Während bei dem bipolaren Widerstandsschalten der Einfluss eines statischen elektrischen Feldes analysiert wird, werden in Kap. 7 die Ergebnisse der Anregungen der Manganatprobe mittels Laserlicht präsentiert. Dabei wird der photoinduzierte Einfluss auf die elektrischen Transporteigenschaften charakterisiert. Als Messgrößen werden die ersten und dritten harmonischen Spannungen aufgezeichnet, aus denen die Konzentration von korrelierten Polaronen berechnet werden kann. Diese Spannungen werden als Funktion der Temperatur und der Laseranregung aufgenommen. Um bei verschiedenen Temperaturen messen zu können, wurde ein Aufbau aus Peltierelementen entwickelt und die Messungen an einer LBMO-Schicht durchgeführt, deren Metall-Isolator-Übergangstemperatur in der Nähe von Raumtemperatur liegt. Als Laser werden ein Diodenlaser im CW-Betrieb und ein Femtosekundenlaser zur Anregung der dünnen Manganatschichten verwendet. 
Die temperaturabhängigen, photoinduzierten Messungen zeigen zusätzliche Nichtlinearitäten, die möglicherweise durch eine veränderte Konzentration der korrelierten Polaronen erklärt werden können. Hiernach kommt es zu einer durch das Laserlicht induzierten Veränderung der lokalen Struktur. Zusätzlich zeigen temperatur- und laserleistungsabhängige Messungen eine Veränderung der ersten harmonischen Spannung mit unterschiedlichen Zeitkonstanten.

Die vorgestellten Experimente werfen entsprechend viele neue hieran anschließende Fragestellungen auf. Als grundlegende Untersuchung muss eine systematische Analyse der Laserleistungen, der Wellenlängen und der Unterschied zwischen CW-Betrieb und Femtosekundenpulsen auf die Transporteigenschaften folgen. Weiterführende Experimente betreffen außerdem die Analyse der (ultrakurzen) Zeitabhängigkeit der elektrischen Transportphänomene, insbesondere der dritten harmonischen Spannung. Die in dieser Arbeit vorgestellten Messungen sind somit Wegbereiter für viele weiterführende Experimente, in denen mit einer photoinduzierten Anregung der wechselseitige Einfluss der elektronischen Korrelationen mit der Struktur systematisch untersucht werden kann.

Eine detaillierte Analyse des Einflusses der verschiedenen externen Stimuli wie ein elektrisches Feld und Licht auf die Eigenschaften der Manganate ist von fundamentalem Interesse für das Verständnis der stark korrelierten Elektronensysteme. Im Rahmen dieser Arbeit werden einige Aspekte der Manganatphysik untersucht und somit wichtige Fragen beantwortet - allerdings ergeben sich in diesem Zusammenhang wiederum viele neue und spannende Fragestellungen. Damit ist am Schluss dieser Arbeit nun der Zeitpunkt gekommen, den Aufruf „ENTER THE OXIDES“ [1] zu wiederholen und weitere Wissenschaftler dazu aufzufordern, das faszinierende Wechselspiel der elektronischen, magnetischen und strukturellen Freiheitsgrade etwa auf dem Gebiet der Manganate zu erforschen. 



\section{Literatur}

[1] J. Heber. Enter the oxides. Nature, 459, 28 (2009).

[2] H. Y. Hwang, Y. Iwasa, M. Kawasaki, B. Keimer, N. Nagaosa \& Y. ToKURA. Emergent phenomena at oxide interfaces. Nature Materials, 11, 103 (2012).

[3] A. Оhтомо \& H. Y. Hwang. A high-mobility electron gas at the $\mathrm{LaAlO}_{3} / \mathrm{SrTiO}_{3}$ heterointerface. Nature, 427, 423 (2004).

[4] N. Reyren, S. Thiel, A. D. Caviglia, L. Fitting Kourkoutis, G. HamMerl, C. Richter, C. W. Schneider, T. Kopp, A.-S. Rüetschi, D. Jaccard, M. Gabay, D. A. Muller, J.-M. Triscone \& J. Mannhart. Superconducting interfaces between insulating oxides. Science, 317, 1196 (2007).

[5] Editorial. The oxide is still the device. Nature Materials, 11, 91 (2012).

[6] M. J. Calderón, J. Salafranca \& L. Brey. Electron gas at the interface between two antiferromagnetic insulating manganites. Phys. Rev. B, 78, 024415 (2008).

[7] K. Miyano, T. Tanaka, Y. Tomioka \& Y. Tokura. Photoinduced insulatorto-metal transition in a perovskite manganite. Phys. Rev. Lett., 78, 4257 (1997).

[8] M. Fiebig, K. Miyano, Y. Tomioka \& Y. Tokura. Visualization of the local insulator-metal transition in $\mathrm{Pr}_{0.7} \mathrm{Ca}_{0.3} \mathrm{MnO}_{3}$. Science, 280, 1925 (1998).

[9] A. Asamitsu, Y. Tomioka, H. Kuwahara \& Y. Tokura. Current switching of resistive states in magnetoresistive manganites. Nature, 388, 50 (1997).

[10] K.-I. Chahara, T. Ohno, M. Kasai \& Y. Kozono. Magnetoresistance in magnetic manganese oxide with intrinsic antiferromagnetic spin structure. Appl. Phys. Lett., 63, 1990 (1993).

[11] R. von Helmolt, J. Wecker, B. Holzapfel, L. Schultz \& K. Samwer. Giant negative magnetoresistance in perovskitelike $\mathrm{La}_{2 / 3} \mathrm{Ba}_{1 / 3} \mathrm{MnO}_{x}$ ferromagnetic films. Phys. Rev. Lett., 71, 2331 (1993).

[12] H. L. Ju, C. Kwon, Q. Li, R. L. Greene \& T. Venkatesan. Giant magnetoresistance in $\mathrm{La}_{1-x} \mathrm{Sr}_{x} \mathrm{MnO}_{z}$ films near room temperature. Appl. Phys. Lett., 65, 2108 (1994).

[13] S. Jin, T. H. Tiefel, M. McCormack, R. A. Fastnacht, R. Ramesh \& L. H. Chen. Thousandfold change in resistivity in magnetoresistive La-Ca-Mn-O films. Science, 264, 413 (1994).

[14] S. Q. Liu, N. J. Wu \& A. Ignatiev. Electric-pulse-induced reversible resistance change effect in magnetoresistive films. Appl. Phys. Lett., 76, 2749 (2000).

[15] A. Baikalov, Y. Q. Wang, B. Shen, B. Lorenz, S. Tsui, Y. Y. Sun, Y. Y. Xue \& C. W. Chu. Field-driven hysteretic and reversible resistive switch at the Ag- $\mathrm{Pr}_{0.7} \mathrm{Ca}_{0.3} \mathrm{MnO}_{3}$ interface. Appl. Phys. Lett., 83, 957 (2003). 
[16] A. SAwA. Resistive switching in transition metal oxides. Materials today, 11, 28 (2008).

[17] R. Waser \& M. Aono. Nanoionics-based resistive switching memories. Nature Materials, 6, 833 (2007).

[18] Y. Watanabe, J. G. Bednorz, A. Bietsch, C. Gerber, D. Widmer, A. Beck \& S. J. WIND. Current-driven insulator-conductor transition and nonvolatile memory in chromium-doped $\mathrm{SrTiO}_{3}$ single crystals. Appl. Phys. Lett., 78, 3738 (2001).

[19] K. Szot, W. Speier, G. Bihlmayer \& R. Waser. Switching the electrical resistance of individual dislocations in single-crystalline $\mathrm{SrTiO}_{3}$. Nature Materials, 5, $312(2006)$.

[20] A. Beck, J. G. Bednorz, C. Gerber, C. Rossel \& D. Widmer. Reproducible switching effect in thin oxide films for memory applications. Appl. Phys. Lett., 77, 139 (2000).

[21] A. Kingon. Perovskites: Is the ultimate memory in sight? Nature Materials, 5, 251 (2006).

[22] V. Moshnyaga, K. Gehrke, O. I. Lebedev, L. Sudheendra, A. Belenchuk, S. RaAbe, O. Shapoval, J. Verbeeck, G. Van Tendeloo \& K. Samwer. Electrical nonlinearity in colossal magnetoresistance manganite films: Relevance of correlated polarons. Phys. Rev. B, 79, 134413 (2009).

[23] J.-H. Park, E. Vescovo, H.-J. Kim, C. Kwon, R. Ramesh \& T. Venkatesan. Direct evidence for a half-metallic ferromagnet. Nature, 392, 794 (1998).

[24] R. Gross \& A. Marx. Spinelektronik - Vorlesungsskript zur Vorlesung im SS 2004 (2004). Stand 29.01.2013.

URL http://www.wmi.badw.de/teaching/Lecturenotes/index.html

[25] E. Dagotto. Nanoscale phase separation and colossal magnetoresistance - the physics of manganites and related compounds. Springer (2003).

[26] Y. TokuRa (Editor). Colossal magnetoresistive oxides. Gordon and Breach Science Publishers (2000).

[27] R. D. Shannon. Revised effective ionic radii and systematic studies of interatomic distances in halides and chalcogenides. Acta Crystallographica Section A, 32, 751 (1976).

[28] Y. Tokura \& Y. Tomioka. Colossal magnetoresistive manganites. Journal of Magnetism and Magnetic Materials, 200, 1 (1999).

[29] J. F. Mitchell, D. N. Argyriou, C. D. Potter, D. G. Hinks, J. D. JorgenSEN \& S. D. BADER. Structural phase diagram of $\mathrm{La}_{1-x} \mathrm{Sr}_{x} \mathrm{MnO}_{3+\delta}$ : Relationship to magnetic and transport properties. Phys. Rev. B, 54, 6172 (1996).

[30] S. Blundell. Magnetism in condensed matter. Oxford University Press (2001).

[31] P. W. Anderson \& H. Hasegawa. Considerations on double exchange. Phys. Rev., 100, 675 (1955).

[32] A. J. Millis, P. B. Littlewood \& B. I. Shraiman. Double exchange alone does not explain the resistivity of $\mathrm{La}_{1-x} \mathrm{Sr}_{x} \mathrm{MnO}_{3}$. Phys. Rev. Lett., 74, 5144 (1995). 
[33] G.-M. Zhao, K. Conder, H. Keller \& K. A. MÜller. Giant oxygen isotope shift in the magnetoresistive perovskite $\mathrm{La}_{1-x} \mathrm{Ca}_{x} \mathrm{MnO}_{3+y}$. Nature, 381, 676 (1996).

[34] T. Akimoto, Y. Maruyama, Y. Moritomo, A. Nakamura, K. Hirota, K. Ohoyama \& M. Ohashi. Antiferromagnetic metallic state in doped manganites. Phys. Rev. B, 57, R5594 (1998).

[35] W. Westhäuser, S. Schramm, J. Hoffmann \& C. Jooss. Comparative study of magnetic and electric field induced insulator-metal-transitions in $\operatorname{Pr}_{1-x} \mathrm{Ca}_{x} \mathrm{MnO}_{3}$ films. EPJ B, 53, 323 (2006).

[36] C. Jooss, L. Wu, T. Beetz, R. F. Klie, M. Beleggia, M. A. Schofield, S. Schramm, J. Hoffmann \& Y. Zhu. Polaron melting and ordering as key mechanisms for colossal resistance effects in manganites. PNAS, 104, 13597 (2007).

[37] P. Dai, J. A. Fernandez-Baca, N. Wakabayashi, E. W. Plummer, Y. ToMIOKA \& Y. TOKURA. Short-range polaron correlations in the ferromagnetic $\mathrm{La}_{1-x} \mathrm{Ca}_{x} \mathrm{MnO}_{3}$. Phys. Rev. Lett., 85, 2553 (2000).

[38] C. P. Adams, J. W. Lynn, Y. M. Mukovskit, A. A. Arsenov \& D. A. ShulyateV. Charge ordering and polaron formation in the magnetoresistive oxide $\mathrm{La}_{0.7} \mathrm{Ca}_{0.3} \mathrm{MnO}_{3}$. Phys. Rev. Lett., 85, 3954 (2000).

[39] S. Shimomura, T. Tonegawa, K. Tajima, N. Wakabayashi, N. Ikeda, T. Shobu, Y. Noda, Y. Tomioka \& Y. Tokura. X-ray diffuse scattering study on charge-localized states of $\mathrm{Pr}_{1-x} \mathrm{Ca}_{x} \mathrm{MnO}_{3}(x=0.35,0.4,0.5)$. Phys. Rev. B, 62, 3875 (2000).

[40] A. S. Alexandrov \& A. M. Bratkovsky. Carrier density collapse and colossal magnetoresistance in doped manganites. Phys. Rev. Lett., 82, 141 (1999).

[41] A. S. Alexandrov, A. M. Bratkovsky \& V. V. Kabanov. Phase coexistence and resistivity near the ferromagnetic transition of manganites. Phys. Rev. Lett., 96, 117003 (2006).

[42] S. Yunoki, J. Hu, A. L. Malvezzi, A. Moreo, N. Furukawa \& E. Dagotto. Phase separation in electronic models for manganites. Phys. Rev. Lett., 80, 845 (1998).

[43] A. Moreo, S. Yunoki \& E. Dagotto. Phase separation scenario for manganese oxides and related materials. Science, 283, 2034 (1999).

[44] M. Fäth, S. Freisem, A. A. Menovsky, Y. Tomioka, J. Aarts \& J. A. Mydosh. Spatially inhomogeneous metal-insulator transition in doped manganites. Science, 285, 1540 (1999).

[45] M. Uehara, S. Mori, C. H. Chen \& S. W. Cheong. Percolative phase separation underlies colossal magnetoresistance in mixed-valent manganites. Nature, 399, 560 (1999).

[46] M. Mayr, A. Moreo, J. A. Vergés, J. Arispe, A. Feiguin \& E. Dagotto. Resistivity of mixed-phase manganites. Phys. Rev. Lett., 86, 135 (2001).

[47] R. Mathieu, D. Akahoshi, A. Asamitsu, Y. Tomioka \& Y. Tokura. Colossal magnetoresistance without phase separation: Disorder-induced spin glass state and nanometer scale orbital-charge correlation in half doped manganites. Phys. Rev. Lett., 93, 227202 (2004). 
[48] S. A. Köster, V. Moshnyaga, B. Damaschke \& K. Samwer. Nonpercolative behavior of the magnetic-field-induced local tunneling conductivity of $\mathrm{La}_{0.75} \mathrm{Ca}_{0.25} \mathrm{MnO}_{3} / \mathrm{MgO}$ (100) thin films. Phys. Rev. B, 78, 052404 (2008).

[49] V. Moshnyaga, L. Sudheendra, O. I. Lebedev, S. A. Köster, K. Gehrke, O. Shapoval, A. Belenchuk, B. Damaschke, G. van Tendeloo \& K. SamWER. A-site ordering versus electronic inhomogeneity in CMR-manganite films. Phys. Rev. Lett., 97, 107205 (2006).

[50] A. Urushibara, Y. Moritomo, T. Arima, A. Asamitsu, G. Kido \& Y. ToKURA. Insulator-metal transition and giant magnetoresistance in $\mathrm{La}_{1-x} \mathrm{Sr}_{x} \mathrm{MnO}_{3}$. Phys. Rev. B, 51, 14103 (1995).

[51] H. Kawano, R. Kajimoto, M. Kubota \& H. Yoshizawa. Ferromagnetisminduced reentrant structural transition and phase diagram of the lightly doped insulator $\mathrm{La}_{1-x} \mathrm{Sr}_{x} \mathrm{MnO}_{3}(x<\approx 0.17)$. Phys. Rev. B, 53, R14709 (1996).

[52] J. M. D. Coey, M. Viret \& S. von Molnár. Mixed-valence manganites. Advances in Physics, 48, 167 (1999).

[53] R. Waser, R. Dittmann, G. Staikov \& K. Szot. Redox-based resistive switching memories - Nanoionic mechanisms, prospects, and challenges. Advanced $\mathrm{Ma}$ terials, 21, 2632 (2009).

[54] G. I. Meijer. Who wins the nonvolatile memory race? Science, 319, 1625 (2008).

[55] J. Y. Son \& Y.-H. SHIN. Direct observation of conducting filaments on resistive switching of NiO thin films. Appl. Phys. Lett., 92, 222106 (2008).

[56] J. F. Gibbons \& W. E. Beadle. Switching properties of thin Nio films. Solid-State Electronics, 7, 785 (1964).

[57] K. Fujiwara, T. Nemoto, M. J. Rozenberg, Y. Nakamura \& H. Takagi. Resistance switching and formation of a conductive bridge in metal/binary oxide/metal structure for memory devices. Jpn. J. Appl. Phys., 47, 6266 (2008).

[58] H. Sim, H. Choi, D. Lee, M. Chang, D. Choi, Y. Son, E.-H. Lee, W. Kim, Y. PARK, I.-K. Yoo \& H. Hwang. Excellent resistance switching characteristics of $\mathrm{Pt} / \mathrm{SrTiO}_{3}$ Schottky junction for multi-bit nonvolatile memory application. In Electron Devices Meeting, 2005. IEDM Technical Digest. IEEE International (2005), 758.

[59] A. Ignatiev, N. J. Wu, X. Chen, S. Q. Liu, C. Papagianni \& J. Strozier. Resistance switching in perovskite thin films. physica status solidi (b), 243, 2089 (2006).

[60] J.-O. Krisponeit. Untersuchung des elektrischen Widerstandsschaltens perowskitischer Manganatsysteme auf der Nanometerskala. Dissertation, Georg-AugustUniversität Göttingen (2011).

[61] C. Jooss, J. Hoffmann, J. Fladerer, M. Ehrhardt, T. Beetz, L. Wu \& Y. ZHU. Electric pulse induced resistance change effect in manganites due to polaron localization at the metal-oxide interfacial region. Phys. Rev. B, 77, 132409 (2008).

[62] A. Sawa, T. FujiI, M. Kawasaki \& Y. Tokura. Hysteretic current-voltage characteristics and resistance switching at a rectifying $\mathrm{Ti} / \mathrm{Pr}_{0.7} \mathrm{Ca}_{0.3} \mathrm{MnO}_{3}$ interface. Appl. Phys. Lett., 85, 4073 (2004). 
[63] A. Sawa, T. Fujil, M. Kawasaki \& Y. Tokura. Interface resistance switching at a few nanometer thick perovskite manganite active layers. Appl. Phys. Lett., 88, $232112(2006)$.

[64] M. Quintero, P. Levy, A. G. Leyva \& M. J. Rozenberg. Mechanism of electric-pulse-induced resistance switching in manganites. Phys. Rev. Lett., 98, 116601 (2007).

[65] S. Tsui, A. Baikalov, J. Cmaidalka, Y. Y. Sun, Y. Q. Wang, Y. Y. Xue, C. W. Chu, L. Chen \& A. J. Jacobson. Field-induced resistive switching in metal-oxide interfaces. Appl. Phys. Lett., 85, 317 (2004).

[66] W. W. Zhuang, W. Pan, B. D. Ulrich, J. J. Lee, L. Stecker, A. Burmaster, D. R. Evans, S. T. Hsu, M. Tajiri, A. Shimaoka, K. Inoue, T. Naka, N. Awaya, A. Sakiyama, Y. Wang, S. Q. Liu, N. J. Wu \& A. Ignatiev. Novel colossal magnetoresistive thin film nonvolatile resistance random access memory (RRAM). IEDM Tech. Dig., 193 (2002).

[67] D. B. Strukov, G. S. Snider, D. R. Stewart \& R. S. Williams. The missing memristor found. Nature, 453, 80 (2008).

[68] L. ChuA. Memristor - The missing circuit element. IEEE Transactions on Circuit Theory, 18, 507 (1971).

[69] L. O. Chua \& S. O. Kang. Memristive devices and systems. Proceedings of the IEEE, 64, 209 (1976).

[70] T. Ohno, T. Hasegawa, T. Tsuruoka, K. Terabe, J. K. Gimzewski \& M. Aono. Short-term plasticity and long-term potentiation mimicked in single inorganic synapses. Nature Materials, 10, 591 (2011).

[71] V. Moshnyaga, I. Khoroshun, A. Sidorenko, P. Petrenko, A. Weidinger, M. Zeitler, B. Rauschenbach, R. Tidecks \& K. Samwer. Preparation of rare-earth manganite-oxide thin films by metalorganic aerosol deposition technique. Appl. Phys. Lett., 74, 2842 (1999).

[72] V. Moshnyaga. Metal-insulator transition and magnetoresistance in manganite thin films: lattice strain and disorder effects (2004).

[73] K. GenrKe. Magnetoelektrische Eigenschaften von Manganat-Titanat Übergittern. Dissertation, Georg-August-Universität Göttingen (2010).

[74] C. Ballani, S. Hühn, M. Jungbauer, M. Michelmann, F. Massel, O. Shapoval, A. Belenchuk \& V. Moshnyaga. New developments in MAD technique: Layer-by-layer growth of thin oxide films and superlattices (2012), DPG Frühjahrstagung, DS 37.4.

[75] Bruker AXS GmbH. D8 Advance/Discover (D8 Röntgen-Diffraktometer) (20002001).

[76] C. Suryanarayana \& M. Grant Norton. X-ray diffraction: a practical approach. Plenum Press, New York and London (1998).

[77] H. Kiessig. Interferenz von Röntgenstrahlen an dünnen Schichten. Annalen der Physik, 5, 51 (1931).

[78] A. Segmüller. Observation of X-ray interferences on thin films of amorphous silicon. Thin Solid Films, 18, 287 (1973). 
[79] W. Hink \& W. Petzold. X-ray interference patterns of vapor deposited aluminium layers. Zeitschrift für Angewandte Physik, 10, 553 (1958).

[80] Carl Zeiss NTS GmbH. Handbuch für die Rasterelektronenmikroskope SUPRA(VP) und ULTRA (2004).

[81] Quantum Design. Magnetic Property Measurement Systems MPMS XL. Stand 29.01.2013.

URL http://www.qdusa.com/sitedocs/productBrochures/1014-003.pdf

[82] M. MCelfresh. Fundamentals of magnetism and magnetic measurements featuring Quantum Design's magnetic property measurement system. Quantum Design, Purdue University. Stand 29.01.2013.

URL http://www.qdusa.com/sitedocs/appNotes/mpms/FundPrimer.pdf

[83] Quantum Design. MPMS XL - Hardware \& software reference manuals.

[84] C. Enss \& S. Hunklinger. Tieftemperaturphysik. Springer-Verlag (2000).

[85] W. Buckel. Supraleitung, Grundlagen und Anwendungen. VCH-Verlag GmbH, 5. edn. (1994).

[86] R. Gross \& A. Marx. Applied Superconductivity: Josephson Effect and Superconducting Electronics (2005). Stand 29.01.2013.

URL http://www.wmi.badw.de/teaching/Lecturenotes/

[87] S. RAABE. Tunnelmagnetowiderstandseffekte in planaren Tunnelkontakten $\mathrm{La}_{0,7} \mathrm{Sr}_{0,3} \mathrm{MnO}_{3} / \mathrm{MgO} / \mathrm{La}_{0,7} \mathrm{Sr}_{0,3} \mathrm{MnO}_{3}$. Diplomarbeit, Georg-August-Universität Göttingen (2008).

[88] M. Esseling. Grenzflächeneffekte in Manganatschichten. Dissertation, GeorgAugust-Universität Göttingen (2007).

[89] S. Köster. Änderung der Magnetowiderstandseffekte in $\mathrm{La}_{0,7} \mathrm{Sr}_{0,3} \mathrm{MnO}_{3}$-Filmen durch Dotierung der Korngernzen mit MgO. Diplomarbeit, Georg-August-Universität Göttingen (2002).

[90] S. A. Köster, V. Moshnyaga, K. Samwer, O. I. Lebedev, G. van Tendeloo, O. Shapoval \& A. Belenchuk. Doping of interfaces in $\left(\mathrm{La}_{0.7} \mathrm{Sr}_{0.3} \mathrm{MnO}_{3}\right)_{1-x}:(\mathrm{MgO})_{x}$ composite films. Appl. Phys. Lett., 81, 1648 (2002).

[91] A. Kern, R. Doetzer \& W. Eysel. Magnesium oxide. JCPDS-International Center for Diffraction Data (1993).

[92] A. M. Haghiri-Gosnet \& J.-P. Renard. CMR manganites: physics, thin films and devices. J. Phys. D: Appl. Phys., 36, R127 (2003).

[93] V. Moshnyaga, M. Esseling, L. Sudheendra, O. I. Lebedev, K. Gehrke, G. van Tendeloo \& K. SAmWER. Memristor behaviour in nano-sized vertical LSMO/LSMO tunnel junctions. arXiv:1002.0495 (2010).

[94] N. D. Mathur, G. Burnell, S. P. Isaac, T. J. Jackson, B.-S. Teo, J. L. Macmanus-Driscoll, L. F. Cohen, J. E. Evetts \& M. G. Blamire. Large low field magnetoresistance in $\mathrm{La}_{0.7} \mathrm{Ca}_{0.3} \mathrm{MnO}_{3}$ induced by artificial grain boundaries. Nature, 387, 266 (1997). 
[95] J. Fontcuberta, L. Balcells, B. Martínez \& X. Obradors. Magnetoresistance at interfaces in submicrometric manganese perovskite ceramics. (Nano-crystalline and thin film magnetic oxides). I. Nedkov, M. Ausloos (Editors). Kluwer Academic Publishers, 105 (1999).

[96] S. Kar, J. Sarkar, B. Ghosh \& A. K. Raychaudhuri. Spatially resolved study of eletronic transport through grain boundaries in nanostructured films of $\mathrm{La}_{0.67} \mathrm{Sr}_{0.33} \mathrm{MnO}_{3}$. Phys. Rev. B, 74, 085412 (2006).

[97] H. Y. Hwang, S.-W. Cheong, N. P. Ong \& B. Batlogg. Spin-polarized intergrain tunneling in $\mathrm{La}_{2 / 3} \mathrm{Sr}_{1 / 3} \mathrm{MnO}_{3}$. Phys. Rev. Lett., 77, 2041 (1996).

[98] L. Balcells, J. Fontcuberta, B. Martínez \& X. Obradors. High-field magnetoresistance at interfaces in manganese perovskites. Phys. Rev. B, 58, R14697 (1998).

[99] L. Steren, M. Sirena \& J. Guimpel. Substrate effect on the magnetic behaviour of manganite films. J. Appl. Phys., 87, 6755 (2000).

[100] N. Zhang, W. Ding, W. Zhong, D. Xing \& Y. Du. Tunnel-type giant magnetoresistance in the granular perovskite $\mathrm{La}_{0.85} \mathrm{Sr}_{0.15} \mathrm{MnO}_{3}$. Phys. Rev. B, 56, 8138 (1997).

[101] P. Dey \& T. K. NATH. Effect of grain size modulation on the magneto- and electronic-transport properties of $\mathrm{La}_{0.7} \mathrm{Ca}_{0.3} \mathrm{MnO}_{3}$ nanoparticles: The role of spinpolarized tunneling at the enhanced grain surface. Phys. Rev. B, 73, 214425 (2006).

[102] R. D. Sánchez, J. Rivas, C. Vázquez-Vázquez, A. López-Quintela, M. T. Causa, M. Tovar \& S. Oseroff. Giant magnetoresistance in fine particle of $\mathrm{La}_{0.67} \mathrm{Ca}_{0.33} \mathrm{MnO}_{3}$ synthesized at low temperatures. Appl. Phys. Lett., 68, 134 (1996).

[103] J. Rivas, L. E. Hueso, A. Fondado, F. Rivadulla \& M. A. López-Quintela. Low field magnetoresistance effects in fine particles of $\mathrm{La}_{0.67} \mathrm{Ca}_{0.33} \mathrm{MnO}_{3}$ perovskites. Journal of Magnetism and Magnetic Materials, 221, 57 (2000).

[104] T. Zhu, B. G. Shen, J. R. Sun, H. W. Zhao \& W. S. Zhan. Surface spin-glass behavior in $\mathrm{La}_{2 / 3} \mathrm{Sr}_{1 / 3} \mathrm{MnO}_{3}$ nanoparticles. Appl. Phys. Lett., 78, 3863 (2001).

[105] L. Balcells, B. Martínez, F. Sandiumenge \& J. Fontcuberta. Magnetotransport properties of nanometric $\mathrm{La}_{2 / 3} \mathrm{Sr}_{1 / 3} \mathrm{MnO}_{3}$. Journal of Magnetism and Magnetic Materials, 211, 193 (2000).

[106] M. Lanza, K. Zhang, M. Porti, M. Nafría, Z. Y. Shen, L. F. Liu, J. F. Kang, D. Gilmer \& G. Bersuker. Grain boundaries as preferential sites for resistive switching in the $\mathrm{HfO}_{2}$ resistive random access memory structures. Appl. Phys. Lett., 100, 123508 (2012).

[107] R. Muenstermann, T. Menke, R. Dittmann, S. Mi, C.-L. Jia, D. Park \& J. MAYER. Correlation between growth kinetics and nanoscale resistive switching properties of $\mathrm{SrTiO}_{3}$ thin films. J. Appl. Phys., 108, 124504 (2010).

[108] X. Chen, N. J. Wu, J. Strozier \& A. Ignatiev. Spatially extended nature of resistive switching in perovskite oxide thin films. Appl. Phys. Lett., 89, 063507 (2006). 
[109] C. Moreno, C. Munuera, S. Valencia, F. Kronast, X. Obradors \& C. OCAL. Reversible resistive switching and multilevel recording in $\mathrm{La}_{0.7} \mathrm{Sr}_{0.3} \mathrm{MnO}_{3}$ thin films for low cost nonvolatile memories. Nano Lett., 10, 3828 (2010).

[110] Y. W. Xie, J. R. Sun, D. J. Wang, S. Liang \& B. G. Shen. Reversible electroresistance at the $\mathrm{Ag} / \mathrm{La}_{0.67} \mathrm{Sr}_{0.33} \mathrm{MnO}_{3}$ interface. J. Appl. Phys., 100, 033704 (2006).

[111] X. Chen, J. Strozier, N. J. Wu, A. Ignatiev \& Y. B. Nian. A study of the symmetry properties and multi-state nature of perovskite oxide-based electrical pulse induced resistance-change devices. New Journal of Physics, 8, 229 (2006).

[112] M. J. Rozenberg, M. J. Sánchez, R. Weht, C. Acha, F. Gomez-Marlasca \& P. LEVY. Mechanism for bipolar resistive switching in transition-metal oxides. Phys. Rev. B, 81, 115101 (2010).

[113] M. Scherff, B.-U. Meyer, J. Hoffmann \& C. Jooss. Polarity reversal in bipolar resistive switching in $\operatorname{Pr}_{0.7} \mathrm{Ca}_{0.3} \mathrm{MnO}_{3}$ noble metal sandwich structures. $J$. Appl. Phys., 110, 043718 (2011).

[114] C. Yoshida, M. Kurasawa, Y. M. Lee, M. Aoki \& Y. Sugiyama. Unipolar resistive switching in $\mathrm{CoFeB} / \mathrm{MgO} / \mathrm{CoFeB}$ magnetic tunnel junction. Appl. Phys. Lett., 92, 113508 (2008).

[115] P. Krzysteczko, G. Reiss \& A. Thomas. Memristive switching of MgO based magnetic tunnel junctions. Appl. Phys. Lett., 95, 112508 (2009).

[116] D. Halley, H. Majuad, M. Bowen, N. Najuari, Y. Henry, C. UlhaqBoulllet, W. Weber, G. Bertoni, J. Verbeeck \& G. Van Tendeloo. Electrical switching in $\mathrm{Fe} / \mathrm{Cr} / \mathrm{MgO} / \mathrm{Fe}$ magnetic tunnel junctions. Appl. Phys. Lett., 92, 212115 (2008).

[117] S.-S. Chen, C.-P. Yang, L.-F. Xu, F.-J. Yang, H.-B. Wang, H. Wang, L.-B. XIong, Y. Yu, I. Medvedeva \& K. BÄrneR. Electric-pulse-induced resistance switching in $\mathrm{Nd}_{0.7} \mathrm{Sr}_{0.3} \mathrm{MnO}_{3}$ ceramics. Solid State Communications, 150, 240 (2010).

[118] Z. Liao, P. Gao, X. Bai, D. Chen \& J. Zhang. Evidence for electric-field-driven migration and diffusion of oxygen vacancies in $\mathrm{Pr}_{0.7} \mathrm{Ca}_{0.3} \mathrm{MnO}_{3}$. J. Appl. Phys., 111, 114506 (2012).

[119] D. S. Shang, L. D. Chen, Q. Wang, W. Q. Zhang, Z. H. Wu \& X. M. Li. Temperature dependence of current-voltage characteristics of $\mathrm{Ag}-\mathrm{La}_{0.7} \mathrm{Ca}_{0.3} \mathrm{MnO}_{3}$ Pt heterostructures. Appl. Phys. Lett., 89, 172102 (2006).

[120] H. K. Lau \& C. W. Leung. Nonvolatile multilevel memory effect by resistive switching in manganite thin films. J. Appl. Phys., 104, 123705 (2008).

[121] J.-O. Krisponeit, C. Kalkert, B. Damaschke, V. Moshnyaga \& K. SamWER. Nanoscale resistance switching in manganite thin films: Sharp voltage treshold and pulse-width dependence. Phys. Rev. B, 82, 144440 (2010).

[122] T. Sarkar, B. Ghosh, A. K. Raychaudhuri \& T. Chatterji. Crystal structure and physical properties of half-doped manganite nanocrystals of less than 100-nm size. Phys. Rev. B, 77, 235112 (2008). 
[123] O. I. Lebedev, J. Verbeeck, G. Van Tendeloo, O. Shapoval, A. BelenChuk, V. Moshnyaga, B. Damaschke \& K. Samwer. Structural phase transitions and stress accommodation in $\left(\mathrm{La}_{0.67} \mathrm{Ca}_{0.33} \mathrm{MnO}_{3}\right)_{1-x}: \mathrm{MgO}_{x}$ composite films. Phys. Rev. B, 66, 104421 (2002).

[124] V. Moshnyaga, B. Damaschke, O. Shapoval, A. Belenchuk, J. Faupel, O. I. Lebedev, J. Verbeeck, G. van Tendeloo, M. Mücksch, V. Tsurkan, R. Tidecks \& K. SAmwer. Structural phase transition at the percolation threshold in epitaxial $\left(\mathrm{La}_{0.7} \mathrm{Ca}_{0.3} \mathrm{MnO}_{3}\right)_{1-x}:(\mathrm{MgO})_{x}$ nanocomposite films. Nature Materials, 2, 247 (2003).

[125] T. Frolov, D. L. Olmsted, M. Asta \& Y. Mishin. Structural phase transformations in metallic grain boundaries. arXiv:1211.1756 (2012).

[126] W. Preis \& W. SitTe. Fast grain boundary diffusion and rate-limiting surface exchange reactions in polycrystalline materials. J. Appl. Phys., 97, 093504 (2005).

[127] R. A. De Souza, J. A. Kilner \& J. F. Walker. A SiMS study of oxygen tracer diffusion and surface exchange in $\mathrm{La}_{0.8} \mathrm{Sr}_{0.2} \mathrm{MnO}_{3+\delta}$. Materials Letters, 43, 43 (2000).

[128] A. V. Berenov, J. L. Macmanus-Driscoll \& J. A. Kilner. Oxygen tracer diffusion in undoped lanthanum manganites. Solid State Ionics, 122, 41 (1999).

[129] Y. B. Nian, J. Strozier, N. J. Wu, X. Chen \& A. Ignatiev. Evidence for an oxygen diffusion model for the electric pulse induced resistance change effect in transition-metal oxides. Phys. Rev. Lett., 98, 146403 (2007).

[130] G. Gottstein. Physikalische Grundlagen der Materialkunde. Springer (2007).

[131] R. P. Borges, W. Guichard, J. G. Lunney, J. M. D. Coey \& F. Ott. Magnetic and electric dead layers in $\left(\mathrm{La}_{0,7} \mathrm{Sr}_{0,3}\right) \mathrm{MnO}_{3}$ thin films. J. Appl. Phys., 89, 3868 (2001).

[132] L. Lu, W. Yi \& D. L. ZhAng. $3 \omega$ method for specific heat and thermal conductivity measurements. Rev. Sci. Instrum., 72, 2996 (2001).

[133] M. P. Raphael, M. E. Reeves \& E. F. Skelton. Nonlinear response of type II superconductors: A new method of measuring the pressure dependence of the transition temperature $\mathrm{T}_{c}(\mathrm{P})$. Rev. Sci. Instrum., 69, 1451 (1998).

[134] N. Chéenne, T. Mishonov \& J. O. Indekeu. Observation of a sharp lambda peak in the third harmonic voltage response of high- $\mathrm{T}_{c}$ superconductor thin films. EPJ B, 32, 437 (2003).

[135] J. G. Ossandon, S. Sergeenkov, P. Esquinazi \& H. Kempa. Higher harmonics of ac voltage response in narrow strips of $\mathrm{YBa}_{2} \mathrm{Cu}_{3} \mathrm{O}_{7}$ thin films: evidence for strong thermal fluctuations. Supercond. Sci. Technol., 18, 325 (2005).

[136] Q. Wen \& D. R. Clarke. Electrical harmonic generation from notched narrow metal lines. J. Appl. Phys., 83, 1132 (1998).

[137] A. B. Beznosov, V. A. Desnenko, E. L. Fertman, C. Ritter \& D. D. KhaLYAVIN. Magnetic and neutron diffraction study of $\mathrm{La}_{2} / 3 \mathrm{Ba}_{1 / 3} \mathrm{MnO}_{3}$ perovskite manganite. Phys. Rev. B, 68, 054109 (2003). 
[138] M. Quijada, J. Černe, J. R. Simpson, H. D. Drew, K. H. Ahn, A. J. Millis, R. Shreekala, R. Ramesh, M. Rajeswari \& T. Venkatesan. Optical conductivity of manganites: Crossover from Jahn-Teller small polaron to coherent transport in the ferromagnetic state. Phys. Rev. B, 58, 16093 (1998).

[139] J. F. Lawler, J. G. Lunney \& J. M. D. Coey. Magneto-optic Faraday effect in $\left(\mathrm{La}_{1-x} \mathrm{Ca}_{x}\right) \mathrm{MnO}_{3}$ films. Appl. Phys. Lett., 65, 3017 (1994).

[140] V. N. Smolyaninova, G. Yong, R. M. Kolagani, A. Biswas, H. K. Ermer, K. WANG \& A. PiAzZA. Large photoinduced conductivity reduction in thin films of metallic ferromagnetic manganites. Appl. Phys. Lett., 99, 222507 (2011).

[141] V. Moshnyaga, A. Giske, K. Samwer, E. Mishina, T. Tamura, S. Nakabayashi, A. Belenchuk, O. Shapoval \& L. Kulyuk. Giant negative photoconductivity in $\mathrm{La}_{0.7} \mathrm{Ca}_{0.3} \mathrm{MnO}_{3}$ thin films. J. Appl. Phys., 95, 7360 (2004). 


\section{Publikationen}

\section{Artikel}

- Nanoscale Resistance SWitching in manganite thin films: Sharp voltage THRESHOLD AND PULSE-WIDTH DEPENDENCE

J.-O. Krisponeit, C. Kalkert, B. Damaschke, V. Moshnyaga und K. Samwer Phys. Rev. B, 82, 144440, 2010.

- Resistive Switching at manganite/manganite interfaces C. Kalkert, J.-O. Krisponeit, M. Esseling, O. I. Lebedev, V. Moshnyaga, B. Damaschke, G. van Tendeloo und K. Samwer

Appl. Phy. Lett., 99, 132512, 2011.

- Time-Resolved Resistive SWitching ON MANGanite SuRfaces: CReEP AND $1 / f^{\alpha}$ Noise signatures indicAte Pinning of nANOSCALE DOMAINS J.-O. Krisponeit, C. Kalkert, B. Damaschke, V. Moshnyaga und K. Samwer Phys. Rev. B: Rapid Communications, 87, 121103(R), 2013.

\section{Konferenzbeiträge}

- International Conference on Magnetism, Karlsruhe 2009.

- Frühjahrstagung der Deutschen Physikalischen Gesellschaft, Dresden, 2009.

- Edgar-Lüscher Seminar, Klosters, Schweiz, 2009.

- Frühjahrstagung der Deutschen Physikalischen Gesellschaft, Regensburg, 2010.

- Frühjahrstagung der Deutschen Physikalischen Gesellschaft, Dresden 2011.

- Edgar-Lüscher Seminar, Klosters, Schweiz, 2011.

- Frühjahrstagung der Deutschen Physikalischen Gesellschaft, Dresden 2012.

- Edgar-Lüscher Seminar, Klosters, Schweiz, 2012. 



\section{Danksagung}

Am Ende dieser Arbeit ist die Zeit gekommen, sich bei den vielen Menschen zu bedanken, die mit ihrer Hilfe und tatkräftiger Unterstützung zum Gelingen dieser Arbeit beigetragen haben.

An erster Stelle möchte ich Prof. Dr. Konrad Samwer einen ganz besonderen Dank dafür aussprechen, dass er mir diese Promotion ermöglicht hat. Die zahlreichen Diskussionen und Ideen haben diese Arbeit immer wieder an den entscheidenden Stellen angestoßen und weiter vorangetrieben. Des Weiteren möchte ich auch dafür Danke sagen, dass ich jedes Jahr wieder an Seminarausflügen und Klosterfahrten teilnehmen konnte. Durch diese Exkursionen hat sich nicht nur mein Verständnis für die weite Welt der Festkörperphysik ständig erweitert, diese Wochen haben auch dazu beigetragen, dass ich mich in meiner Zeit im I. Institut sehr wohl gefühlt habe.

Ein herzliches Dankeschön richtet sich auch an Prof. Dr. Vasily Moshnyaga, der sogar kurzfristig noch das Koreferat dieser Arbeit übernommen hat. Stets war er auch zu langen Diskussion über die faszinierenden Eigenschaften der Manganate bereit und hat mit zahlreichen Ideen, Anregungen und Aufmunterungen zum steten Voranschreiten dieser Arbeit beigetragen.

Bei Dr. Bernd Damaschke möchte ich mich sehr herzlich dafür bedanken, dass er mir bei allen großen und kleinen Fragen jederzeit hilfreich zur Seite gestanden hat. In unzähligen Jour Fixe wurden bei einem guten Kaffee die neusten Messergebnisse diskutiert und Ideen und Problemlösungen entwickelt, auf die diese Arbeit aufgebaut ist.

Die Zusammenarbeit mit Jon-Olaf Krisponeit hat diese Arbeit in vielerlei Hinsicht bereichert. Neben den gemeinsamen C-AFM Messungen möchte ich mich für die zahlreichen Diskussionen und nicht zuletzt für das gründliche Lektorat dieser Arbeit sehr bedanken. Zusätzlich möchte ich Markus Michelmann Danke sagen, der sogar kurzfristig noch einige Kapitel der Arbeit Korrektur gelesen hat. Auch die übrigen Mitglieder der ManganateGruppe müssen an dieser Stelle Erwähnung finden: Hierbei danke ich besonders Sebastian Hühn, Markus Jungbauer und Florian Fischgrabe - mit euch hat die Arbeit immer sehr viel Spaß gemacht.

Für die Zusammenarbeit bei den photoinduzierten Messungen möchte ich mich bei Prof. Markus Münzenberg, Marvin Walter, Jakob Walowski und Manuel Mchalwat bedanken. Die photoinduzierten Messungen sind nur im Rahmen dieser Kooperation möglich gewesen.

Für Implementations-Hilfe bei der Simulation bedanke ich mich bei Rafael Brune: Mit dir hat auch das viele „Pommes essen gehen“ in der Nordmensa immer für Ausgleich gesorgt.

Uta Filipich danke ich für die lustige Zeit und die große Bereitschaft, mir jederzeit hilfreich im Labor (und auch bei sonstigen Fragen) zur Seite zu stehen. Auch ein großes Dankeschön richtet sich an die tolle Hilfe von Carsten Mahn. Des Weiteren möchte ich für die Unterstützung der Werkstatt Danke sagen, im Besonderen hat mir das Werkstattpraktikum neue Einblicke eröffnet und viel Freude gemacht. 
Ein weiteres Dankeschön geht an meine Bürokollegin Melanie Schneider, die sich immer wieder alle meine kleinen und großen Sorgen geduldig anhören musste. Nicht nur durch die vielen Grünpflanzen, die mit ihr eingezogen sind, hat sie dem Büro eine angenehme Atmosphäre verliehen.

Natürlich möchte ich dem ganzen I. Physikalischen Institut Danke sagen - es hat mir viel Spaß und Freude bereitet, in diesem Umfeld zu arbeiten! Das herzliche Miteinander und das Wissen bei Fragen und Problemen jederzeit einen kompetenten Ansprechpartner zu finden, haben mir meine Arbeit sehr erleichtert.

Auch eine Reihe an Nichtphysikern hat entschieden zum Gelingen dieser Arbeit beigetragen. Ich möchte mich bei meinen Eltern bedanken, die mich immer und in allen Lebenslagen unterstützen. Meiner Schwester Bernadette danke ich für das professionelle Lektorat dieser Arbeit - und die leckeren Mittagessen, wenn ich mal wieder in Köln gearbeitet habe. Richard danke ich für die große Unterstützung und Geduld - und für die vielen gefahrenen Kilometer zwischen Göttingen und Gleuel. 


\section{Lebenslauf}

\section{Persönliche Daten}

$\begin{array}{ll}\text { Name: } & \text { Christin Kalkert } \\ \text { Geburtsdatum: } & \text { 30. Septenber 1983 } \\ \text { Geburtsort: } & \text { Leverkusen } \\ \text { Familienstand: } & \text { ledig } \\ \text { Staatsangehörigkeit: } & \text { deutsch }\end{array}$

\section{Schulbildung}

$\begin{array}{ll}1990-1993 & \text { Porter-Gaud-School, South Carolina, USA } \\ 1993-1994 & \text { Grundschule Sollingstrasse, Holzminden } \\ 1994-1996 & \text { Astrid-Lindgren Orientierungsschule, Holzminden } \\ 1996-2003 & \text { Campe-Gymnasium, Holzminden }\end{array}$

\section{Hochschulausbildung}

$2003-2005$

$2005-2006$

$2006-2008$

seit 2009
Grundstudium Physik, Georg-August-Universität Göttingen

Auslandsjahr an der University of California, San Diego, USA

Hauptstudium Physik, Georg-August-Universität Göttingen Diplomarbeit zum Thema:

„Metall-Isolator-Übergang eines (La,Pr,Ca)-Manganats

- ein Vergleich von globalen und lokalen Eigenschaften"

Wissenschaftliche Mitarbeit im I. Physikalischen Institut der Georg-August-Universität, Göttingen 